National Water-Quality Assessment Project

\title{
Groundwater-Quality and Select Quality-Control Data from the National Water-Quality Assessment Project, January through December 2016, and Previously Unpublished Data from 2013 to 2015
}

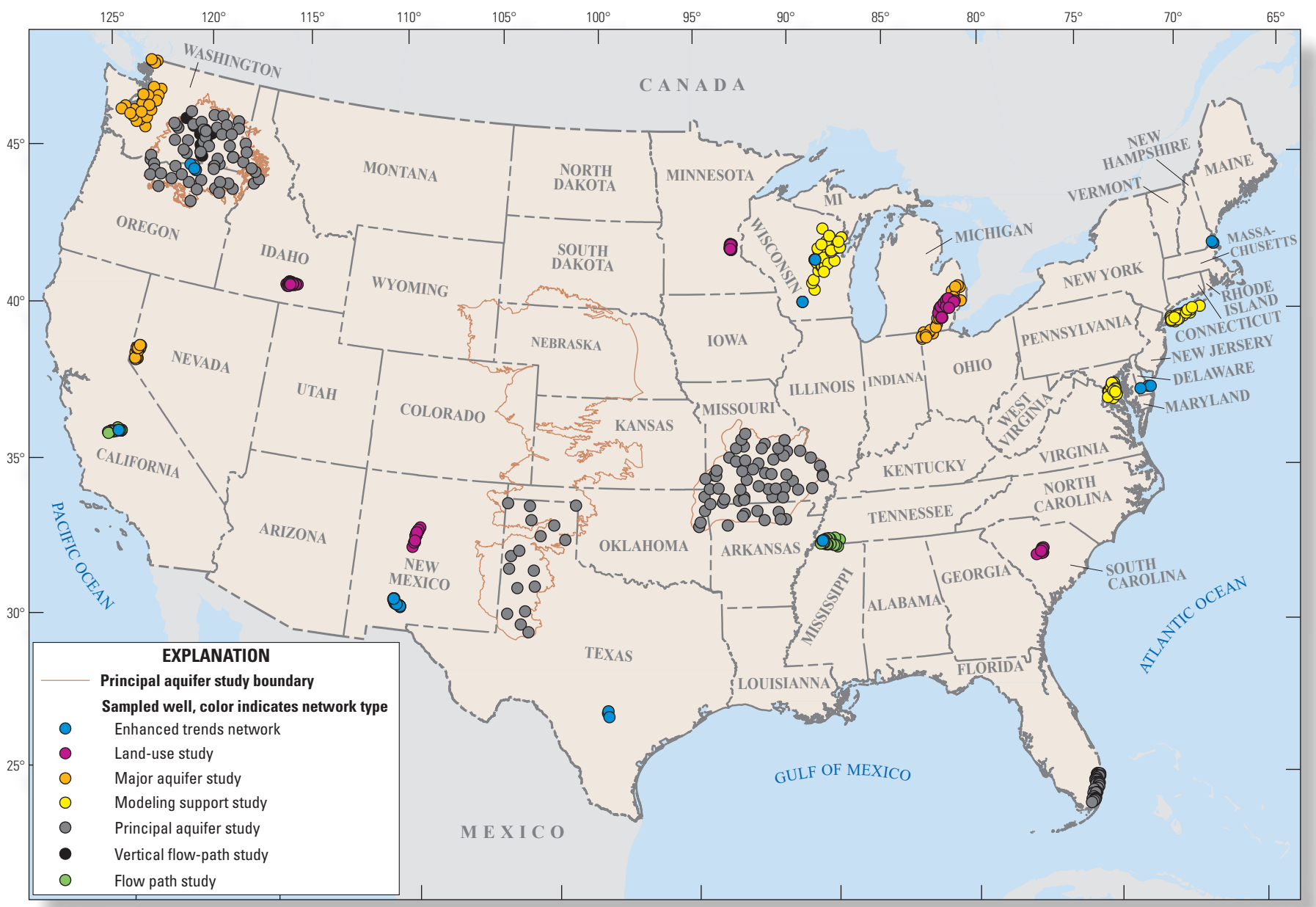

Data Series 1124

U.S. Department of the Interior

U.S. Geological Survey 



\section{Groundwater-Quality and Select Quality-Control Data from the National Water-Quality Assessment Project, January through December 2016, and Previously Unpublished Data from 2013 to 2015}

By Terri L. Arnold, Laura M. Bexfield, MaryLynn Musgrove, Melinda L. Erickson, James A. Kingsbury, James R. Degnan, Anthony J. Tesoriero, Justin T. Kulongoski and Kenneth Belitz

National Water-Quality Assessment Project

Data Series 1124 


\title{
U.S. Department of the Interior \\ DAVID BERNHARDT, Secretary
}

\author{
U.S. Geological Survey \\ James F. Reilly II, Director
}

U.S. Geological Survey, Reston, Virginia: 2020

For more information on the USGS - the Federal source for science about the Earth, its natural and living resources, natural hazards, and the environment—visit https://www.usgs.gov or call 1-888-ASK-USGS.

For an overview of USGS information products, including maps, imagery, and publications, visit https://store.usgs.gov/.

Any use of trade, firm, or product names is for descriptive purposes only and does not imply endorsement by the U.S. Government.

Although this information product, for the most part, is in the public domain, it also may contain copyrighted materials as noted in the text. Permission to reproduce copyrighted items must be secured from the copyright owner.

Suggested citation:

Arnold, T.L., Bexfield, L.M., Musgrove, M., Erickson, M.L., Kingsbury, J.A., Degnan, J.R., Tesoriero, A.J., Kulongoski, J.T., and Belitz, K., 2020, Groundwater-quality and select quality-control data from the National Water-Quality Assessment Project, January through December 2016, and previously unpublished data from 2013 to 2015: U.S. Geological Survey Data Series 1124, 135 p., https://doi.org/10.3133/ds1124.

Associated data for this publication:

Arnold, T.L., Bexfield, L.M., Musgrove, M., Stackelberg, P.E., Lindsey, B.D., Kingsbury, J.A., Kulongoski, J.T., Belitz, K., and Sharpe, J.B., 2020, Datasets from groundwater-quality and select quality-control data from the National WaterQuality Assessment Project, January through December 2016, and previously unpublished data from 2013 to 2015: U.S. Geological Survey data release, accessed February 2020 at https://doi.org/10.5066/P9W4RR74.

U.S. Geological Survey [USGS], 2018, USGS water data for the Nation: U.S. Geological Survey National Water Information System database, accessed December 18, 2018, at https://doi.org/10.5066/F7P55KJN.

ISSN 2327-638X (online) 


\section{Foreword}

Sustaining the quality of the Nation's water resources and the health of our diverse ecosystems depends on the availability of sound water-resources data and information to develop effective, science-based policies. Effective management of water resources also brings more certainty and efficiency to important economic sectors. Taken together, these actions lead to immediate and long-term economic, social, and environmental benefits that make a difference to the lives of the almost 400 million people projected to live in the United States by 2050.

In 1991, Congress established the National Water-Quality Assessment (NAWQA) to address where, when, why, and how the Nation's water quality has changed, or is likely to change in the future, in response to human activities and natural factors. Since then, NAWQA has been a leading source of scientific data and knowledge used by national, regional, state, and local agencies to develop science-based policies and management strategies to improve and protect water resources used for drinking water, recreation, irrigation, energy development, and ecosystem needs (https://water.usgs.gov/nawqa/applications/). Plans for the third decade of NAWQA (2013-23) address priority water-quality issues and science needs identified by NAWQA stakeholders, such as the Advisory Committee on Water Information and the National Research Council, and are designed to meet increasing challenges related to population growth, increasing needs for clean water, and changing land-use and weather patterns.

NAWQA is assessing the quality of groundwater used for public and domestic drinking-water supply. NAWQA obtains samples from public supply wells, domestic wells, and relatively shallow monitoring wells, and analyzes those samples for a large number of chemical constituents. These data are used to assess the suitability of the resource for human consumption, as well as to evaluate changes in groundwater quality over a variety of time scales. Groundwater quality also is assessed at multiple scales: locally, regionally, and nationally. Groundwater-quality data collected by the NAWQA Project during each year are published in annual data series reports. This report, the fourth in the series, combines groundwater-quality data collected at 648 sites to provide a summary of groundwater quality in selected aquifers across the Nation during the sampling period. All NAWQA reports are available online at https://water.usgs.gov/nawqa/bib/.

We hope this publication will provide you with insights and information to meet your waterresource needs and will foster increased citizen awareness and involvement in the protection and restoration of our Nation's waters. The information in this report is intended primarily for those interested or involved in resource management and protection, conservation, regulation, and policymaking at the regional and national levels.

Dr. Donald W. Cline Associate Director for Water

U.S. Geological Survey 



\section{Contents}

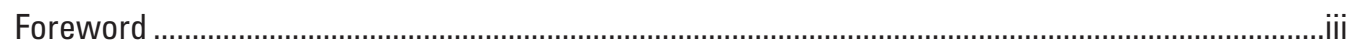

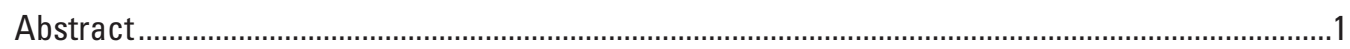

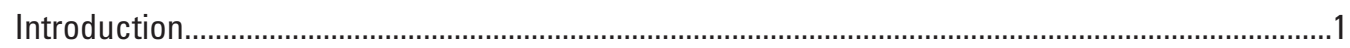

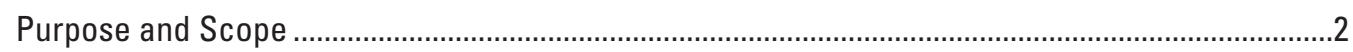

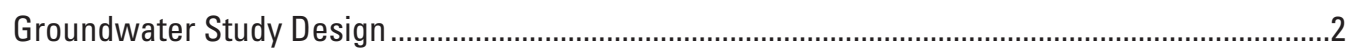

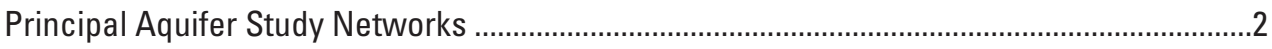

Biscayne Aquifer Principal Aquifer Study Network (biscpas1) .......................................

Columbia Plateau Basaltic-Rock Aquifers Principal Aquifer Study Network

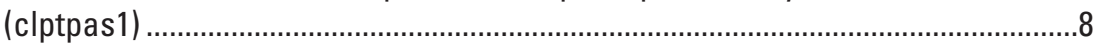

High Plains Aquifer Principal Aquifer Study Network (hpaqpas1) ................................9

Ozark Plateaus Aquifer System Principal Aquifer Study Network (ozrkpas1) .................9

Decadal Trends Networks—Land-Use Study Networks ......................................................

Lake Erie-Lake St. Clair drainages urban land-use study network (lerilusrc1) ................9

Rio Grande Aquifer System Agricultural Land-Use Study Network (rioglusag1) ...........16

Rio Grande aquifer system urban land-use study network (rioglusrc1)........................16

Santee River Basin and Coastal Drainages Urban Land-Use Study Network

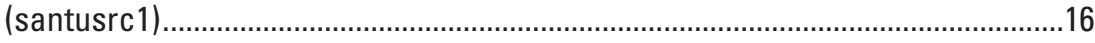

Upper Mississippi River Basin Urban Land-Use Study Network (umislusrc1)................16

Upper Snake River Basin Agricultural Land-Use Study Network (usnkluscr2) ..............17

Decadal Trends Networks_-Major Aquifer Study Networks................................................17

Lake Erie-Lake St. Clair Drainages Major Aquifer Study Network (lerisus1) ..................17

Nevada Basin and Range Major Aquifer Study Network (nvbrsus2) .............................17

Puget Sound Drainages Major Aquifer Study Network (pugtsus1) ..................................17

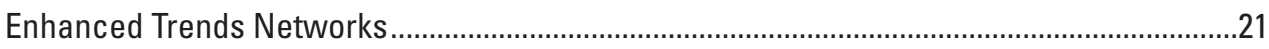

Central Valley Enhanced Trends Network (cvaletn1) .....................................................21

Columbia Plateau Enhanced Trends Network (clptetn1) ..................................................21

Edwards-Trinity Aquifer System Enhanced Trends Network (edtretn1).........................24

Glacial Aquifer System Enhanced Trends Network (glacetn1).......................................24

Mississippi Embayment Aquifer System Enhanced Trends Network (metxetn1) ...........24

Northern Atlantic Coastal Plain Enhanced Trends Network (nacpetn1)........................24

New England Crystalline-Rock and Glacial Aquifer System Enhanced Trends

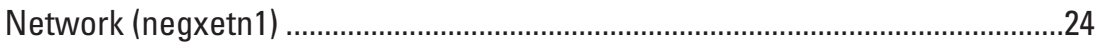

Rio Grande Aquifer System Enhanced Trends Network (rgaqetn1)...............................25

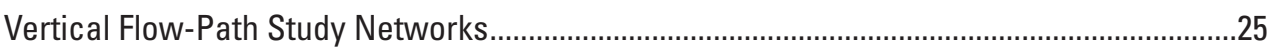

Columbia Plateau Vertical Flow-Path Study Network (clptvfps1) .................................25

Glacial Aquifer System Vertical Flow-Path Study Network (glacvfps1)........................25

Rio Grande Aquifer System Vertical Flow-Path Study Network (rgaqvfps1).................25

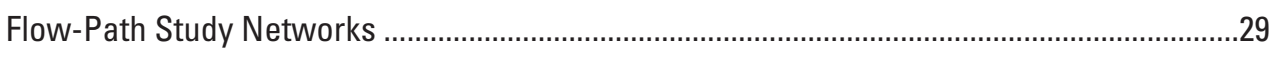

Central Valley Aquifer System Flow-Path Study Networks (cvalfps1, cvalfps2)............29

Glacial Aquifer System Flow-Path Study Network (glacfps1)......................................29

Mississippi Embayment Aquifer System Flow-Path Study Network (metxfps1)............29 


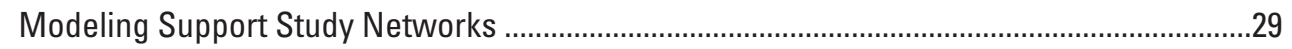

Glacial Aquifer System Modeling Support Study Network (glacmss1) .........................37

Northern Atlantic Coastal Plain Modeling Support Study Networks (nacpmss1,

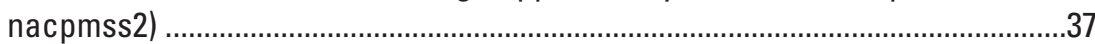

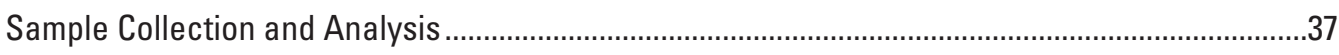

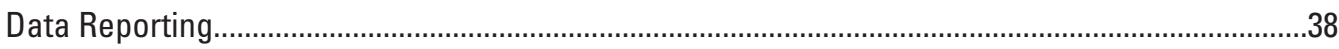

Quality-Assurance and Quality-Control Methods .......................................................................39

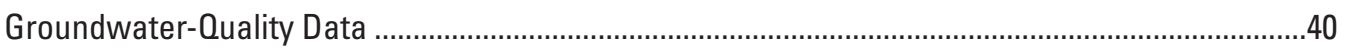

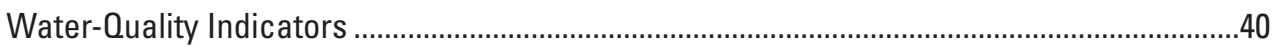

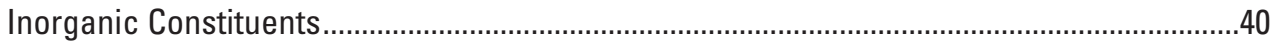

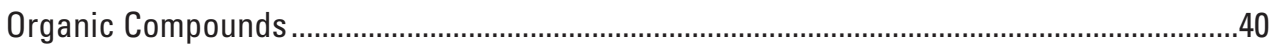

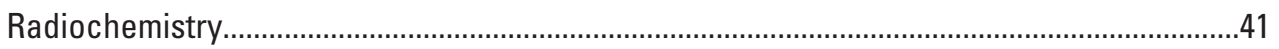

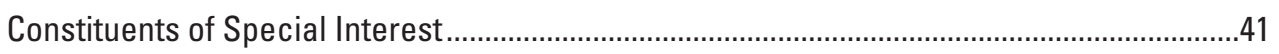

Summary

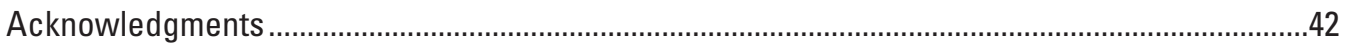

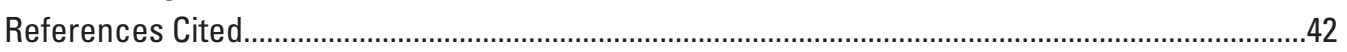

Appendix 1. Information Contained in Previous Reports in This Series......................................103

Appendix 2. Well Depth and Open Interval by Study Network ....................................................108

Appendix 3. High-Frequency Data from Enhanced Trends Networks........................................110

Appendix 4. Quality-Control Samples and Data Analysis ...........................................................112

\section{Figures}

1. Map showing groundwater study networks and wells sampled as part of the U.S. Geological Survey National Water-Quality Assessment Project for which water-quality data are included in this report.

2. Map showing study area and wells sampled as part of the Biscayne aquifer principal aquifer study network

3. Map showing study area and wells sampled as part of the Columbia Plateau Basaltic-Rock Aquifers principal aquifer study network

4. Map showing study area and wells sampled as part of the High Plains aquifer system principal aquifer study network.

5. Map showing study area and wells sampled as part of the Ozark Plateaus aquifer system principal aquifer study network

6. Map showing study area and wells sampled as part of the Lake Erie-Lake St. Clair drainages urban land-use study network

7. Map showing study area and wells sampled as part of the Rio Grande aquifer system agricultural land-use study network.

8. Map showing study area and wells sampled as part of the Rio Grande aquifer system urban land-use study network.

9. Map showing study area and wells sampled as part of the Santee River Basin and Coastal Drainages urban land-use study network

10. Map showing study area and wells sampled as part of the Upper Mississippi River Basin urban land-use study network

11. Map showing study area and wells sampled as part of the Upper Snake River Basin agricultural land-use study network 
12. Map showing study area and wells sampled as part of the Lake Erie-Lake St. Clair drainages major aquifer study network

13. Map showing study area and wells sampled as part of the Nevada Basin and Range major aquifer study network

14. Map showing study area and wells sampled as part of the Puget Sound drainages major aquifer study network.

15. Maps showing study areas and wells sampled in the western United States as part of the enhanced trends networks.

16. Maps showing study areas and wells sampled in the eastern United States as part of the enhanced trends networks

17. Map showing study and wells sampled as part of the Columbia Plateau vertical flow-path study network.

18. Map showing study and wells sampled as part of the Glacial aquifer system vertical flow-path study network

19. Map showing study and wells sampled as part of the Rio Grande aquifer system vertical flow-path study

20. Map showing study and wells sampled as part of the Central Valley aquifer system flow-path study network

21. Map showing study and wells sampled as part of the Central Valley aquifer system flow-path study network

22. Map showing study and wells sampled as part of the Glacial aquifer system flow-path study network

23. Map showing study and wells sampled as part of the Mississippi Embayment aquifer system flow-path study network

24. Map showing study and wells sampled as part of the Northern Atlantic Coastal Plain modeling support study network

25. Map showing study and wells sampled as part of the Northern Atlantic Coastal Plain modeling support study network

26. Map showing study and wells sampled as part of the Glacial aquifer system modeling support study network.

\section{Table}

1. Information about wells that have environmental data included in this report 


\section{Conversion Factors}

U.S. customary units to International System of Units

\begin{tabular}{|c|c|c|}
\hline Multiply & By & To obtain \\
\hline \multicolumn{3}{|c|}{ Length } \\
\hline inch (in.) & 2.54 & centimeter $(\mathrm{cm})$ \\
\hline foot $(\mathrm{ft})$ & 0.3048 & meter $(\mathrm{m})$ \\
\hline mile (mi) & 1.609 & kilometer $(\mathrm{km})$ \\
\hline \multicolumn{3}{|c|}{ Area } \\
\hline square mile $\left(\mathrm{mi}^{2}\right)$ & 2.590 & square kilometer $\left(\mathrm{km}^{2}\right)$ \\
\hline \multicolumn{3}{|c|}{ Radioactivity } \\
\hline picocurie per liter $(\mathrm{pCi} / \mathrm{L})$ & 0.037 & becquerel per liter $(\mathrm{Bq} / \mathrm{L})$ \\
\hline
\end{tabular}

\section{Datum}

Vertical coordinate information is referenced to either the North American Vertical Datum of 1988 (NAVD 88) or the National Geodetic Vertical Datum of 1929 (NGVD 29) and is specified in tables where vertical datum is reported.

Horizontal coordinate information is referenced to the North American Datum of 1983 (NAD 83).

Altitude, as used in this report, refers to distance above the vertical datum.

\section{Supplemental Information}

Specific conductance is given in microsiemens per centimeter at 25 degrees Celsius $(\mu \mathrm{S} / \mathrm{cm}$ at $25^{\circ} \mathrm{C}$ ).

Concentrations of chemical constituents in water are given in either milligrams per liter (mg/L) or micrograms per liter $(\mu \mathrm{g} / \mathrm{L})$.

Activities for radioactive constituents in water are given in picocuries per liter (pCi/L). 


\section{Abbreviations}

$\begin{array}{ll}\text { ASCII } & \text { American Standard Code for Information Interchange } \\ \text { DL } & \text { detection limit } \\ \text { EPA } & \text { U.S. Environmental Protection Agency } \\ \text { ETN } & \text { enhanced trends network } \\ \text { FPS } & \text { flow-path study } \\ \text { HHB } & \text { human-health benchmark } \\ \text { HHBP } & \text { human-health benchmark for pesticides } \\ \text { ID } & \text { identification } \\ \text { LUS } & \text { land-use study } \\ \text { MAS } & \text { major aquifer study } \\ \text { MRL } & \text { minimum reporting level } \\ \text { MSS } & \text { modeling support study } \\ \text { NAWOA } & \text { National Water-Quality Assessment } \\ \text { NWOL } & \text { National Water Quality Laboratory } \\ \text { PAS } & \text { principal aquifer study } \\ \text { OC } & \text { quality control } \\ \text { SSL } & \text { sample-specific critical level } \\ \text { SSMDC } & \text { sample-specific minimum detectable concentration } \\ \text { USGS } & \text { U.S. Geological Survey } \\ \text { VFPS } & \text { vertical flow-path study } \\ \text { VOC } & \text { volatile organic compound }\end{array}$





\title{
Groundwater-Quality and Select Quality-Control Data from the National Water-Quality Assessment Project, January through December 2016, and Previously Unpublished Data from 2013 to 2015
}

\author{
By Terri L. Arnold, Laura M. Bexfield, MaryLynn Musgrove, Melinda L. Erickson, James A. Kingsbury, James \\ R. Degnan, Anthony J. Tesoriero, Justin T. Kulongoski and Kenneth Belitz
}

\section{Abstract}

Environmental groundwater-quality data were collected from 648 wells as part of the National Water-Quality Assessment Project of the U.S. Geological Survey National Water-Quality Program and are included in this report. Most of the wells (514) were sampled from January through December 2016, and 60 of them were sampled in 2013 and 74 in 2014. The data were collected from seven types of well networks: principal aquifer study networks, which are used to assess the quality of groundwater used for public-water supply; land-use study networks, which are used to assess land-use effects on shallow groundwater quality; major aquifer study networks, which are used to assess the quality of groundwater used for domestic supply; enhanced trends networks, which are used to evaluate the time scales during which groundwater quality changes; vertical flow-path study networks, which are used to evaluate changes in groundwater quality from shallow to deeper depths; flow-path study networks, which are used to evaluate changes in groundwater quality from shallow to deeper depths over a horizontal distance; and modeling support studies, which are used to provide data to support groundwater modeling. Groundwater samples were analyzed for many water-quality indicators and constituents, including major ions, nutrients, trace elements, volatile organic compounds, pesticides, radionuclides, and some constituents of special interest (arsenic speciation, chromium [VI], and perchlorate). These groundwater-quality data, along with data from quality-control samples, are tabulated in this report and in an associated data release. Some data from environmental samples collected in 2013-14 and quality-control samples collected in 2012-15 also are included in the associated data release. Data from samples collected in 2016 are associated with networks described in this report and have not been published previously; data from samples collected between 2012 and 2015 are associated with networks described in previous reports in this data series.

\section{Introduction}

The National Water-Quality Assessment (NAWQA) Project of the U.S. Geological Survey (USGS) National WaterQuality Project was fully implemented in 1991 and operates in about 10-year cycles. The NAWQA Project began its third cycle of studies in 2013. The NAWQA Project was designed to describe current water-quality conditions of the Nation's freshwater streams, rivers, and aquifers; to describe how water quality is changing with time; to improve understanding of the natural and human factors that affect water quality; to forecast future water-quality conditions; and to assess effects of waterquality stressors on aquatic ecosystems (Rowe and others, 2010, 2013).

The NAWQA Project groundwater assessments focus on the quality of groundwater used for public and domestic drinking-water supply; groundwater susceptibility to degradation; effects of natural and human factors on source, transport, and flux of contaminants to and within aquifers; groundwaterquality contributions to surface-water quality; and current and previous management practices relative to groundwater quality. Groundwater quality is studied at multiple scales: locally, regionally, and nationally. The primary regional scale at which groundwater data were collected during the third cycle of the NAWQA Project was the scale of the principal aquifers (Burow and Belitz, 2014). A principal aquifer is defined as a regionally extensive aquifer or aquifer system that has the potential to be used as a source of potable water. Principal aquifers were selected for assessment based on their national ranking as sources of water used for public supply (Arnold and others, 2016b).

Groundwater-quality data collected by the NAWQA Project during each year are published in data series reports. The first three reports and associated data releases in this series published available data from samples collected May 2012 through December 2013 (Arnold and others, 2016a,b), January through December 2014 (Arnold and others, 2017a,b), and January through December 2015 (Arnold and others, 
2018a,b). Appendix 1, table 1.1 lists the networks that are described in this report and that are described in previous reports in this series (Arnold and others, 2016a,b, 2017a,b, 2018a,b).

\section{Purpose and Scope}

The purpose of this report is to present the analytical results of the groundwater-quality samples collected in 2016 as part of the third cycle of NAWQA Project studies and to provide brief descriptions of the groundwater-quality study networks for use in subsequent publications. Types of constituents analyzed include the following: water-quality indicators, major and minor ions, nutrients, volatile organic compounds (VOCs), pesticides, radionuclides, and select constituents of special interest (arsenic speciation, chromium [VI], and perchlorate). The water-quality data are presented in tables formatted as tab-delimited American Standard Code for Information Interchange (ASCII) text files, which may be imported into spreadsheet, database, or statistical software for manipulation and analysis. These water-quality data tables are available from a data release, Arnold and others (2020), at https://doi.org/10.5066/P9W4RR74. The data release includes data collected during 2016 and previously unpublished data from 2012 to 2015 sampling. These previously unpublished data are associated with networks described in previous reports (Arnold and others, 2016a,b, 2017a,b, 2018a,b).

\section{Groundwater Study Design}

Groundwater-quality samples were collected from wells that were organized into networks (fig. 1) for study purposes. A network is a group of wells that have been selected for sampling based on specific hydrogeologic conditions, land use, or other design criteria. Many networks have wells that were sampled in multiple decadal sampling periods; however, if a network well was damaged or destroyed, had too little water, or the current owner would not permit sampling, then that well was not resampled during 2016. Maps and tables in this report and in Arnold and others (2020) have well identification (ID) numbers assigned by the NAWQA Project to identify the wells; because some wells could not be resampled, some networks do not have consecutively numbered NAWQA Project IDs. As used on maps showing network-specific information (figs. 2-26), the ID numbers are shown either as numbers only or a combination of numbers and letters that indicate a particular well within the network. The NAWQA well ID number listed in table 1 of this report and table 1 of Arnold and others (2020) are a combination of the network name and the NAWQA Project ID. Data from seven primary types of groundwater study networks are presented in this report (fig. 1): principal aquifer study (PAS), land-use study (LUS), major aquifer study (MAS), enhanced trends networks (ETN), vertical flow-path study (VFPS), flow-path study (FPS), and modeling support study (MSS).

Wells in PAS, LUS, and MAS networks were selected randomly using an equal-area grid to divide the study area of each network (Scott, 1990) into equal-area cells. The equalarea grid method allows for evaluation of constituent concentrations at a regional scale (Belitz and others, 2010). For LUS networks, random potential sampling locations in each grid cell were generated by a software program (Scott, 1990), and monitoring wells were subsequently installed as near to the randomly selected locations as possible. Study areas for LUS networks included the areal extents of the primary aquifer and a specific land use (for example, orchard) of interest. For MAS and PAS networks, one well per grid cell was randomly selected from a population of existing domestic or publicsupply wells (Gilliom and others, 1995; Scott, 1990). For PAS networks, if no public-supply well was available within a grid cell (for example, because permission to sample could not be obtained), an additional well was selected within an adjacent grid cell, not to exceed four wells in two adjacent grid cells. Equal-area grids used for network design are shown only on figures relating to PAS networks because the grids are not available for LUS or MAS networks designed during the first two decades of sampling.

The ETN, VFPS, FPS, and MSS wells were selected from existing networks where possible. The ETN wells are in hydrogeologic settings where changes in hydrologic conditions, land use, or contaminant inputs are expected to be reflected quickly in groundwater (less than 10 years). The VFPS wells were "nested" wells with various depths collocated in a selected area to represent vertical gradients of groundwater flow to enhance the understanding of how contaminants move through aquifers over timespans of greater than a decade. The FPS wells were selected along a horizontal flow path. The MSS wells were selected to provide key data to support specific modeling goals.

\section{Principal Aquifer Study Networks}

The PAS networks consist of public-supply wells, and water is sampled from the part of the aquifer used for the public drinking-water supply (Burow and Belitz, 2014). Publicsupply wells are generally the deepest wells sampled. Wells in PAS networks are sampled once to assess groundwater-quality conditions in the study areas. The extents of PAS network areas are based on the USGS (2003) map of principal aquifers and may be modified in some areas, as described in this report. Data from the following PAS networks are included in this report: Biscayne aquifer PAS network (biscpas1; fig. 2), Columbia Plateau Basaltic-Rock Aquifers PAS network (clptpas1; fig. 3), High Plains aquifer system PAS network (hpaqpas1; fig. 4), and Ozark Plateaus aquifer system PAS network (ozrkpas1; fig. 5). 


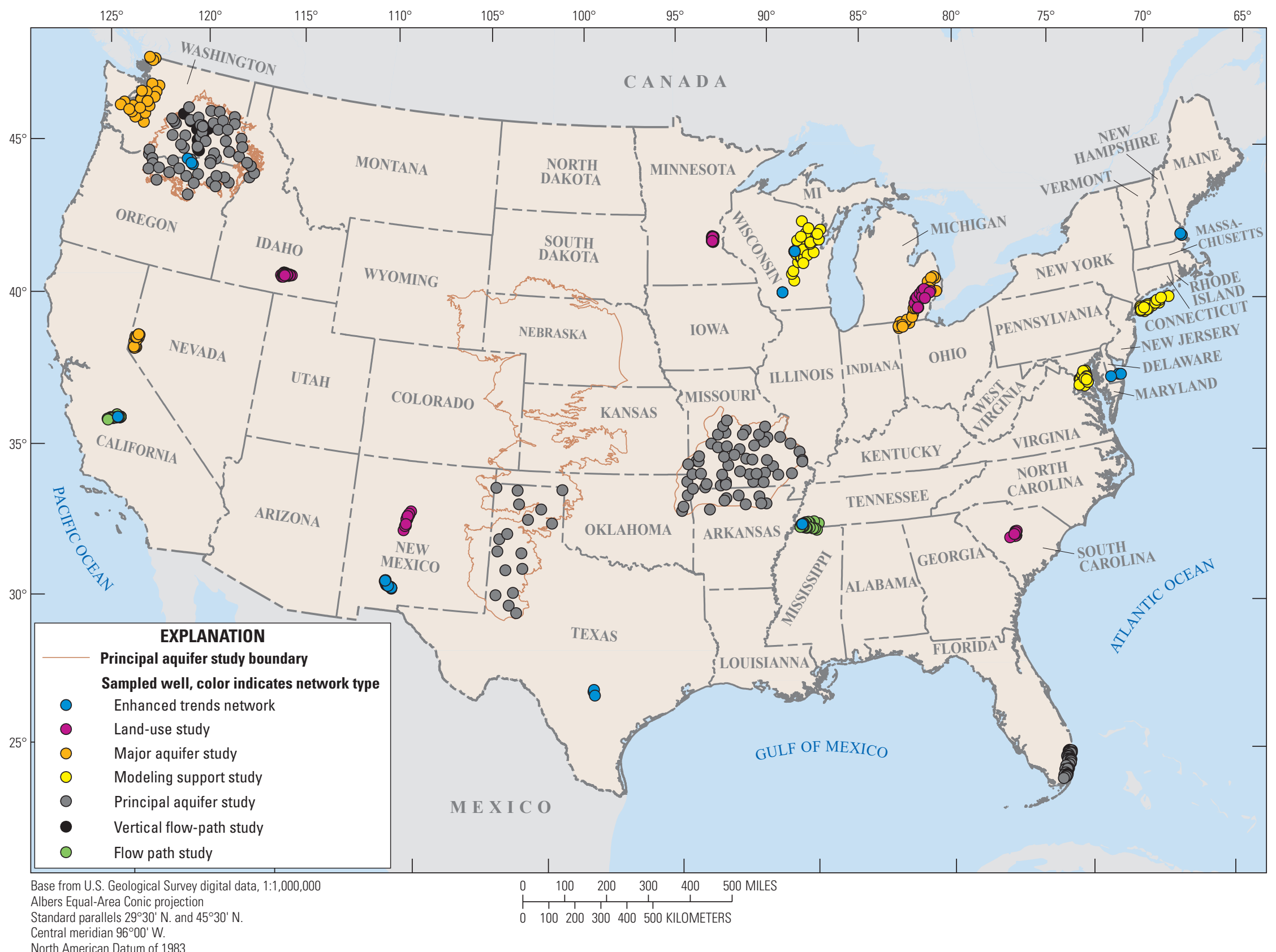

Figure 1. Groundwater study networks and wells sampled as part of the U.S. Geological Survey National Water-Quality Assessment Project for which water-quality data are included in this report.. 


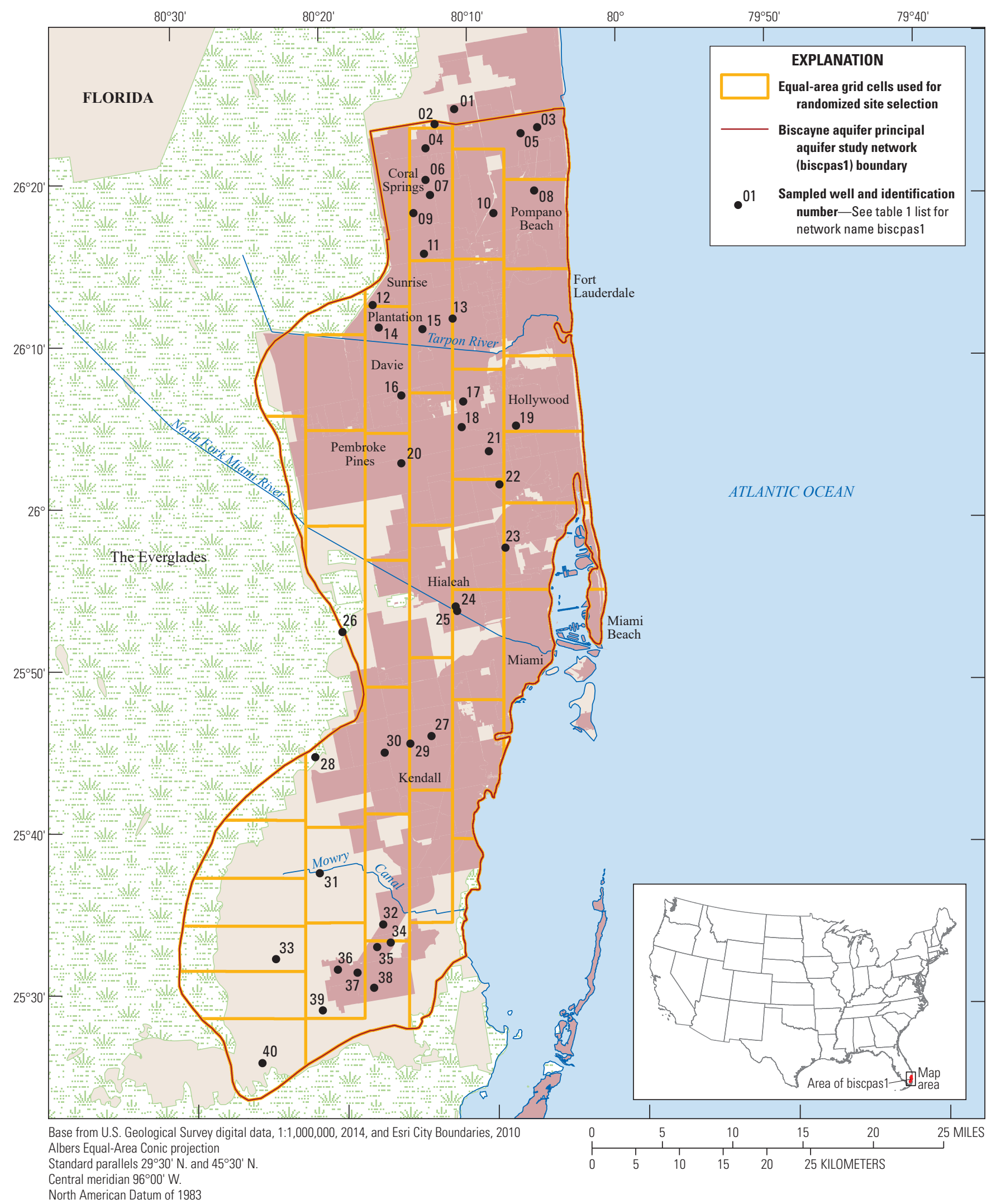

Figure 2. Study area and wells sampled as part of the Biscayne aquifer principal aquifer study network (biscpas1) for the U.S. Geological Survey National Water-Quality Assessment Project. 


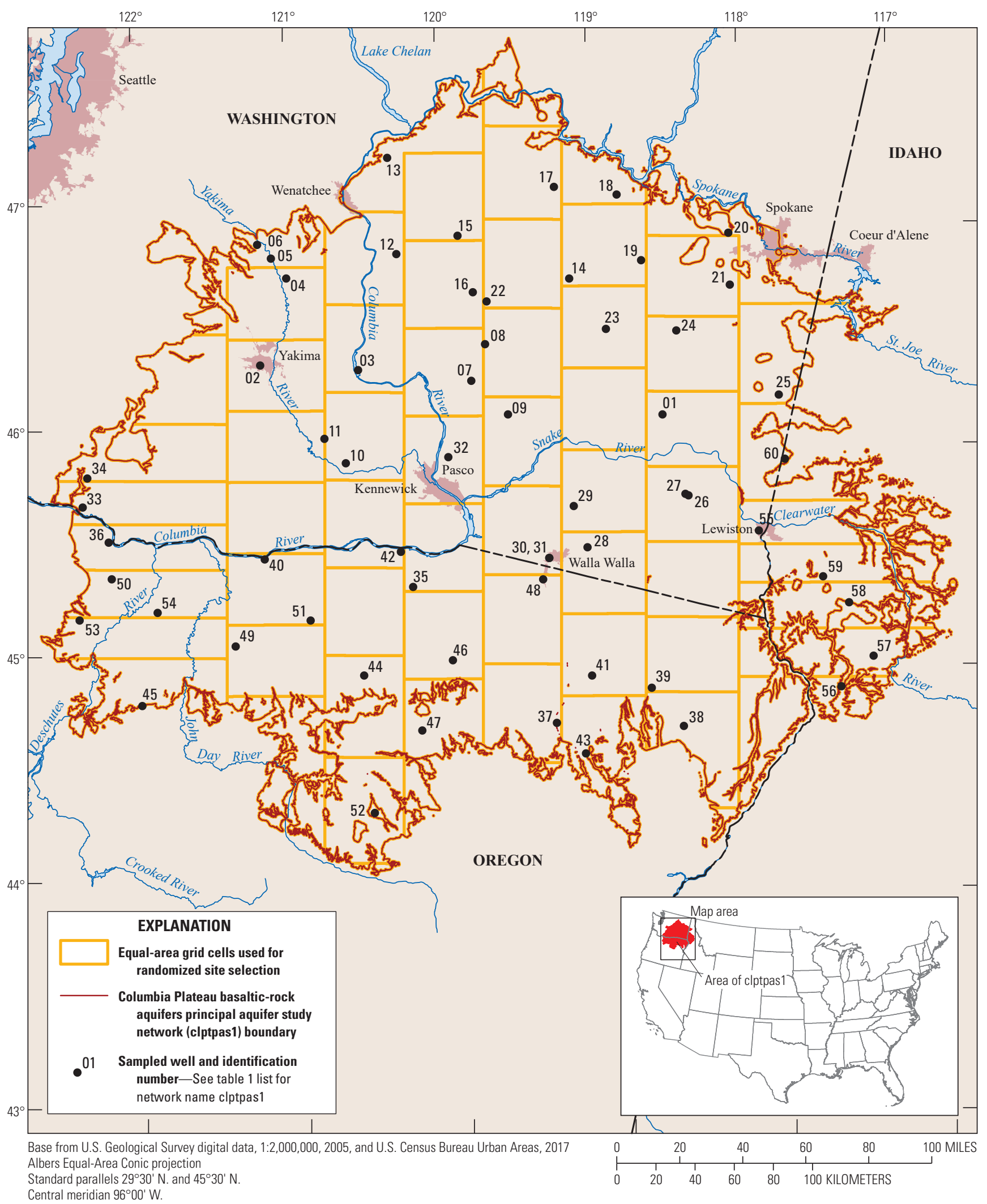

dian $96^{\circ} 00^{\prime} \mathrm{W}$.

Figure 3. Study area and wells sampled as part of the Columbia Plateau Basaltic-Rock Aquifers principal aquifer study network (clptpas1) for the U.S. Geological Survey National Water-Quality Assessment Project. 


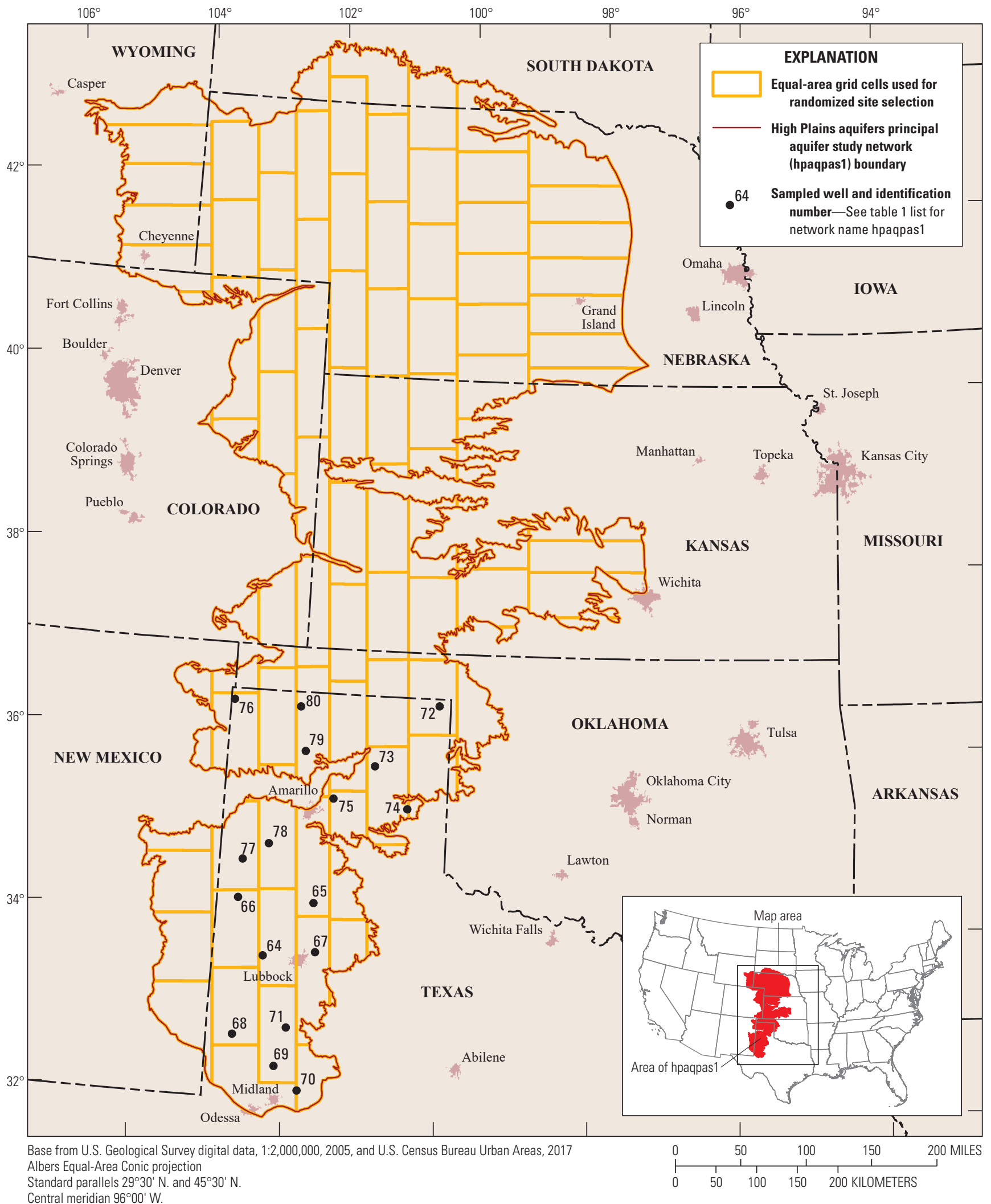

Central meridian $96^{\circ} 00^{\prime} \mathrm{W}$.

Figure 4. Study area and wells sampled as part of the High Plains aquifer system principal aquifer study network (hpaqpas1) for the U.S. Geological Survey National Water-Quality Assessment Project. 


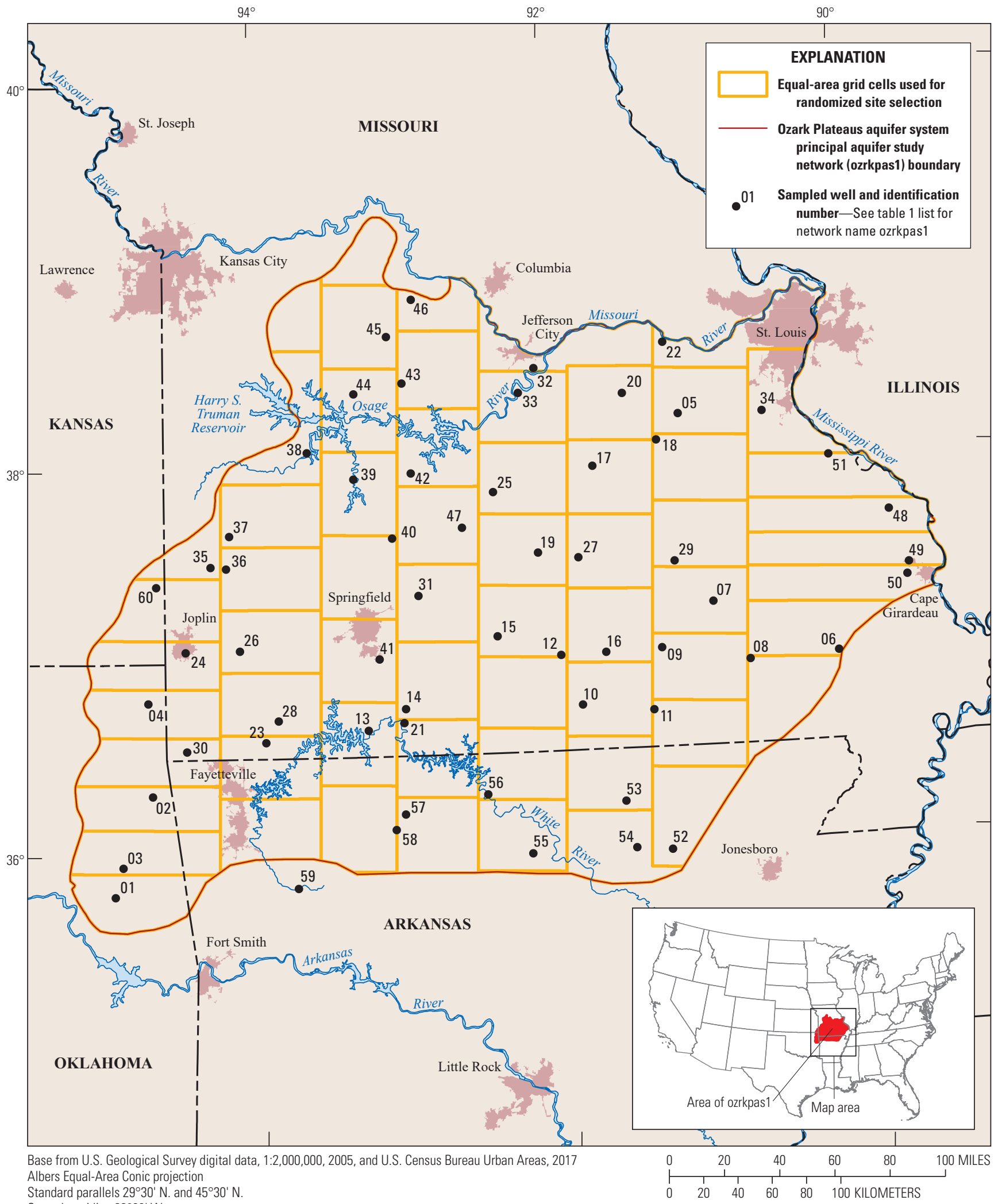

Standard parallels $29^{\circ} 30^{\prime} \mathrm{N}$. and $45^{\circ} 30^{\prime} \mathrm{N}$.

Central meridian $96^{\circ} 00^{\prime} \mathrm{W}$.

North American Datum of 1983

Figure 5. Study area and wells sampled as part of the Ozark Plateaus aquifer system principal aquifer study network (ozrkpas1) for the U.S. Geological Survey National Water-Quality Assessment Project. 


\section{Biscayne Aquifer Principal Aquifer Study Network (biscpas1)}

The Biscayne aquifer underlies an area of about 4,000 square miles $\left(\mathrm{mi}^{2}\right)$ (Miller, 1990), which has a population of about 4 million people in southeastern Florida (U.S. Census Bureau, 2010). About 700 million gallons per day $(\mathrm{Mgal} / \mathrm{d})$ were withdrawn for public supply in 2000 (Maupin and Barber, 2005). The aquifer underlies parts of four (Broward, Miami-Dade, Palm Beach, Monroe) counties in southern Florida, which include the cities of Miami and Ft. Lauderdale. Most of the area overlying the aquifer is developed and consists of urban (63 percent) and agricultural (9 percent) land use (Homer and others, 2015).

The Biscayne aquifer is an unconfined aquifer made up of shallow, highly permeable limestone as well as some sandstone units (Miller, 1990). Because of the shallow depth of the units that make up this aquifer, the hydrologic connection to surface water is an important aspect of the hydrogeology of the Biscayne aquifer (Miller, 1990). A system of canals and levees are used to manage the freshwater resources of southern Florida. The system conserves freshwater, provides flood control, and minimizes saltwater encroachment in the aquifer along the coast (Miller, 1990). Recharge to the aquifer is from seepage of surface water from canals and from infiltration of rainfall (Miller, 1990). Groundwater generally flows from the west to the east, discharging in the Atlantic Ocean but where large withdrawals occur, flow directions have been locally modified (Miller, 1990).

The Biscayne aquifer PAS network (biscpas1; fig. 2) includes 40 public-supply wells distributed across the extent of the aquifer. Wells were selected using a 40-cell equal-area grid that extended across the extent of the aquifer system. Samples were collected between June and September 2016, and the data for these samples are presented in this report. Wells in the biscpas1 ranged from about 22 to 152 feet (ft) deep, with a median well depth of about $91 \mathrm{ft}$. (appendix 2, table 2.1).

\section{Columbia Plateau Basaltic-Rock Aquifers Principal Aquifer Study Network (clptpas1)}

The Columbia Plateau basaltic-rock aquifers underlie an area of 42,000 $\mathrm{mi}^{2}$, which includes about 1 million people in Washington, Oregon, and Idaho (U.S. Census Bureau, 2010). The aquifer system ranks 19th in the Nation as a source of groundwater for public supply, and about $223 \mathrm{Mgal} / \mathrm{d}$ were pumped for this use in 2000 (Maupin and Barber, 2005). Land use overlying the Columbia Plateau basaltic rock aquifers is primarily natural land cover (64 percent) and agriculture (33 percent) with small amounts of urban and other developed land (4 percent) (Homer and others, 2015). The urban areas of Spokane, Washington, and the Washington Tri-Cities (Richland, Kennewick, and Pasco); Moscow and Lewiston,
Idaho; and Pendleton and Hermiston, Oregon, lie within the aquifer system's overlying land along with dozens of smaller communities.

Most of the Columbia Plateau is semiarid (precipitation ranges from 7 to 15 inches per year in the central part of the Plateau), yet the region supports a \$6-billion-per-year agricultural industry, which leads the Nation in production of apples, hops, and several other commodities (Vaccaro and others, 2015). Water demand for agriculture, economic development, and ecological needs in the area is high (Kahle and others, 2011). The Plateau accounts for approximately 5 percent of the Nation's irrigated lands, which are supplied by a combination of groundwater pumping and surface-water diversions.

The Columbia Plateau is an intermontane basin lying between the Cascade Range and the Rocky Mountains that is filled with Cenozoic basalt and sediment. The primary aquifers in the region are the Miocene-aged continental flood basalts of the Columbia River Basalt Group and, in places, Neogene basin-fill sediments, which generally overlie the flood basalts (Vaccaro and others, 2015). Only the basalt-rock aquifers of the clptpas1 network were sampled for this study. The Columbia Plateau basin-fill aquifers rank 67th in the Nation as a source of groundwater for public supply with less than $1 \mathrm{Mgal} /$ day pumped for this use in 2000 (Maupin and Barber, 2005). The Columbia River Basalt Group consists of a series of lava flows that erupted from 17 to 6 million years ago. Individual flows range from 10 to more than $300 \mathrm{ft}$ in thickness, and the total thickness of the flows was estimated to be greater than 15,000 ft in some places (Kahle and others, 2011; Vaccaro and others, 2015). The basaltic-rock aquifer consists of a series of productive basalt units, the most extensive of which is the Grande Ronde Basalt, separated by less permeable interbeds that act as confining units in most places (Whitehead, 1994; Kahle and others, 2011). Groundwater flow is from topographically high margins of the plateau toward major surface drainages, including the Columbia and Snake Rivers. The Cascade Range in Oregon and Washington is an important recharge area for the western part of the regional aquifer system, whereas the western Rocky Mountains and Blue Mountains serve as important recharge areas in the eastern and southern extents of the Columbia Plateau, respectively. Locally, groundwater levels in the Columbia Plateau have changed by up to hundreds of feet because of irrigation practices, resulting in increases (recharge from irrigation) and decreases (groundwater pumping).

The Columbia Plateau basaltic-rock aquifers PAS network (clptpas1; fig. 3) includes 60 public-supply wells, which were selected using an equal-area grid of 60 cells. All 60 of the wells that were selected using the equal-area grid were sampled between June and September 2016 and have data presented in this report. Most of these wells ranged in depth from 91 to 2,434 ft, had a median depth of $484 \mathrm{ft}$ (appendix 2, table 2.1), and were open to the aquifer across long intervals ranging up to approximately $1,297 \mathrm{ft}$ (appendix 2, table 2.2). 
One well in this network also was sampled as part of the Columbia Plateau ETN (clptetn1) network (table 1) described later in this report and in Arnold and others (2017a,b).

\section{High Plains Aquifer Principal Aquifer Study Network (hpaqpas1)}

The High Plains aquifer underlies an area of 170,000 $\mathrm{mi}^{2}$, which has a population of about 2 million people in parts of Colorado, Kansas, Nebraska, New Mexico, Oklahoma, Texas, South Dakota, and Wyoming (U.S. Census Bureau, 2010). The High Plains aquifer previously was described in Arnold and others $(2018 \mathrm{a}, \mathrm{b})$, and the description is not repeated in this report.

The High Plains aquifer system PAS network (hpaqpas1, fig. 4) consists of 80 public-supply wells that were selected based on an equal-area grid. The area of each cell was about 2,122 $\mathrm{mi}^{2}$. Data are presented in this report for 17 wells sampled between May and July 2016 in Texas. An additional 63 wells were sampled March and September 2015 in Colorado, Kansas, Nebraska, New Mexico, Oklahoma, and Wyoming; data from the 2015 sampling are available in Arnold and others $(2018 \mathrm{a}, \mathrm{b})$. Wells in the hpaqpas1 that were sampled in 2016 ranged from 180 to $812 \mathrm{ft}$ deep with a median depth of about $321 \mathrm{ft}$ (appendix 2, table 2.1); wells were open to the aquifer across a range of intervals, from 82 to $265 \mathrm{ft}$, with a median interval of about $161 \mathrm{ft}$ (appendix 2, table 2.2).

\section{Ozark Plateaus Aquifer System Principal Aquifer Study Network (ozrkpas1)}

The Ozark Plateaus aquifer system underlies an area of about 49,000 $\mathrm{mi}^{2}$, including much of southern Missouri, northwestern Arkansas, and relatively small parts of Kansas and Oklahoma. About 4 million people reside in areas overlying this aquifer system (U.S. Census Bureau, 2010) and about $212 \mathrm{Mgal} / \mathrm{d}$ of groundwater was withdrawn for public and domestic supply in 2010 (Hays and others, 2016). Land use in the area overlying the Ozark Plateaus aquifer system is primarily agricultural ( 35 percent) and natural land cover (58 percent) with a small percentage (7 percent) of urban and other developed land (Homer and others, 2015); Springfield, Missouri, is the largest city overlying this aquifer system.

The Ozark Plateaus aquifers consists of three regional aquifers that are separated by confining units (Miller and Appel, 1997, Renken, 1998). The primary lithologies of the geologic formations making up the aquifers are limestone and dolomite. The uppermost Springfield aquifer and the intermediate Ozark aquifer are the primary water-bearing units in this aquifer system. The lowermost St. Francois aquifer has limited use in Arkansas because of its depth and the availability of water in the shallower aquifers and is used primarily where it is near the surface in Missouri (Miller and Appel, 1997). The carbonate rocks that make up these aquifers are susceptible to dissolution and, as a result, karst features such as sinkholes, springs, and caves are common across this aquifer system. Recharge to these aquifers is primarily from infiltration of rainfall, but sinkholes provide areas of focused recharge (Renken, 1998).

The Ozark Plateaus aquifer system PAS network (ozrkpas1; fig. 5) includes 60 public-supply wells distributed across the extent of the aquifer system. Wells were selected using a 60-cell equal-area grid that extended across the extent of the aquifer system. Samples were collected between April and August 2016, and the data for these samples are presented in this report. Wells in the ozrkpas 1 ranged from about 370 to $3,420 \mathrm{ft}$ deep and had a median well depth of about $1,115 \mathrm{ft}$. (appendix 2, table 2.1).

\section{Decadal Trends Networks-Land-Use Study Networks}

The LUS networks are designed to facilitate analysis of land-use effects on shallow groundwater quality. Wells in LUS networks are sampled once per decade to assess temporal trends in water quality. Wells in LUS networks typically are shallow and screened near the water table to allow sampling of recently recharged groundwater that may exhibit chemical characteristics indicative of the overlying land use. The LUS areas are determined by the areal extents of the primary aquifer and a targeted overlying land use (Lapham and others, 1995). Data from the following LUS networks are included in this report: Lake Erie-Lake St. Clair drainages urban LUS network (lerilusrc1; fig. 6) near Detroit, Michigan; Rio Grande aquifer system agricultural LUS network (rioglusag1; fig. 7); Rio Grande aquifer system urban LUS network (rioglusrc1; fig. 8) near Albuquerque, New Mexico; Santee River Basin and Coastal Drainages urban LUS network (santusrc1; fig. 9) near Columbia, South Carolina; Upper Mississippi River Basin urban LUS network (umislusrc1; fig. 10) near Minneapolis/St. Paul, Minnesota; and Upper Snake River Basin agricultural LUS network (usnkluscr2; fig. 11).

\section{Lake Erie-Lake St. Clair drainages urban land-use study network (lerilusrc1)}

The Lake Erie-Lake St. Clair drainages urban LUS network (lerilusrc1; fig. 6) was established to assess how recent residential and commercial land use affects the quality of shallow groundwater in the developed area north and west of Detroit, Mich. The lerilusrc1 study area covers about $600 \mathrm{mi}^{2}$ and coincides with the boundary of the lerisus 1 network described later in this report. Discontinuous layers of sand and gravel are present at various depths within the glacial till, and collectively, these layers of sand and gravel are considered an aquifer system that is variably confined (Fleming, 1994). The hydrogeology is complex because of heterogeneity of glacial materials, fracturing in the surficial till, and the network of tile drains. (Thomas, 2000a). 


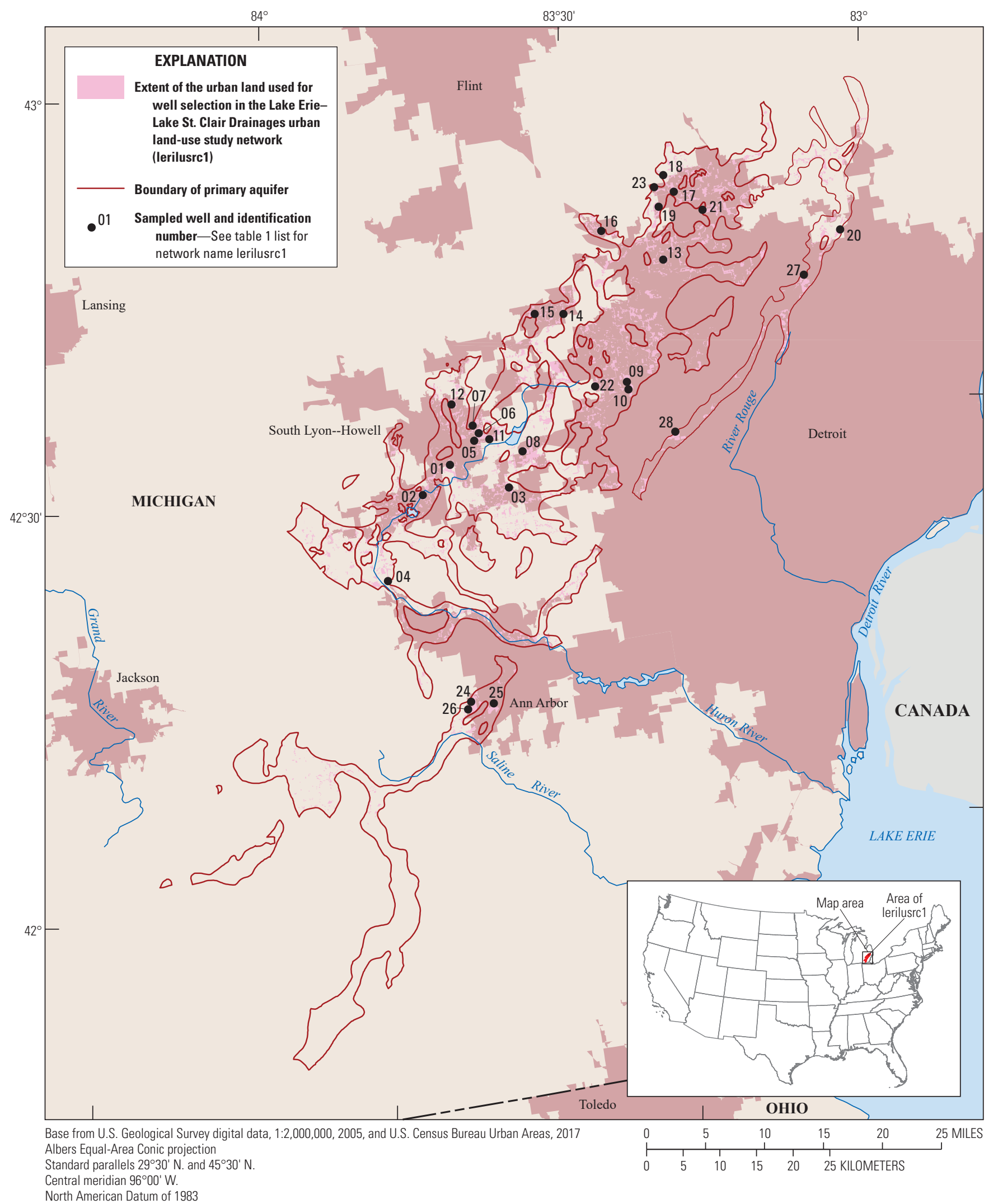

Figure 6. Study area and wells sampled as part of the Lake Erie-Lake St. Clair drainages urban land-use study network (lerilusrc1) near Detroit, Michigan for the U.S. Geological Survey National Water-Quality Assessment Project. 


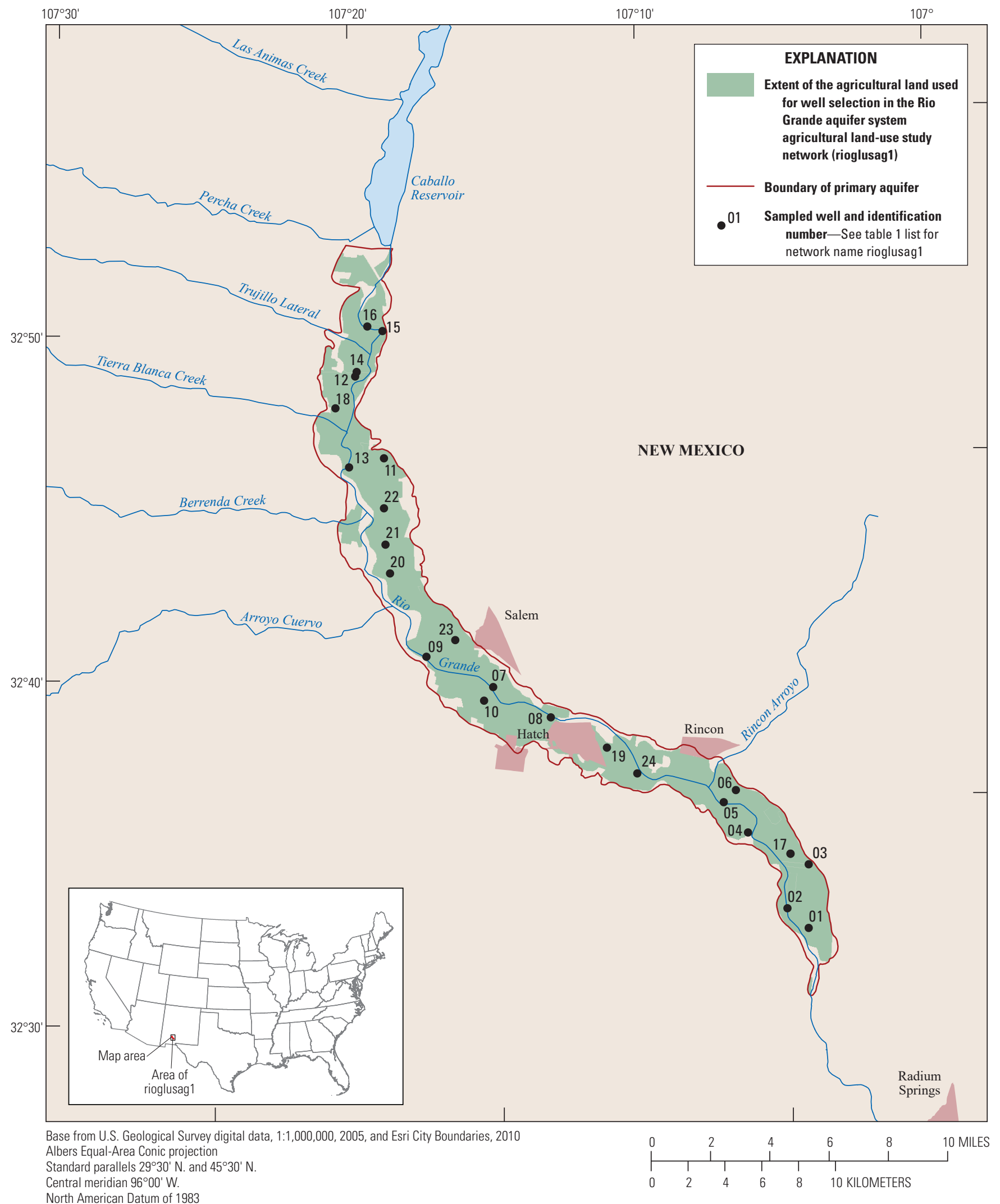

Figure 7. Study area and wells sampled as part of the Rio Grande aquifer system agricultural land-use study network (rioglusag1) for the U.S. Geological Survey National Water-Quality Assessment Project. 


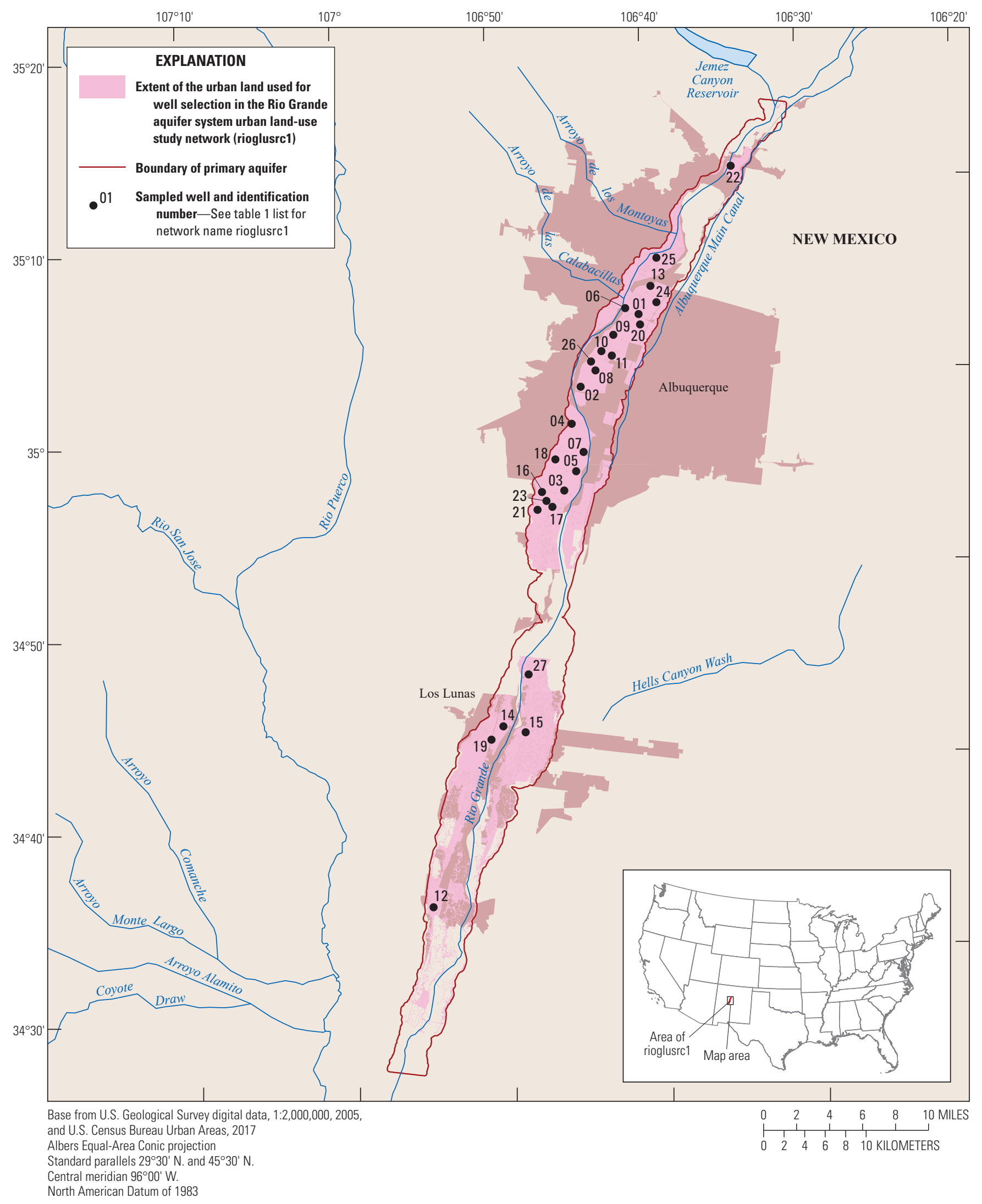

Figure 8. Study area and wells sampled as part of the Rio Grande aquifer system urban land-use study network (rioglusrc1) near Albuquerque, New Mexico for the U.S. Geological Survey National Water-Quality Assessment Project. 


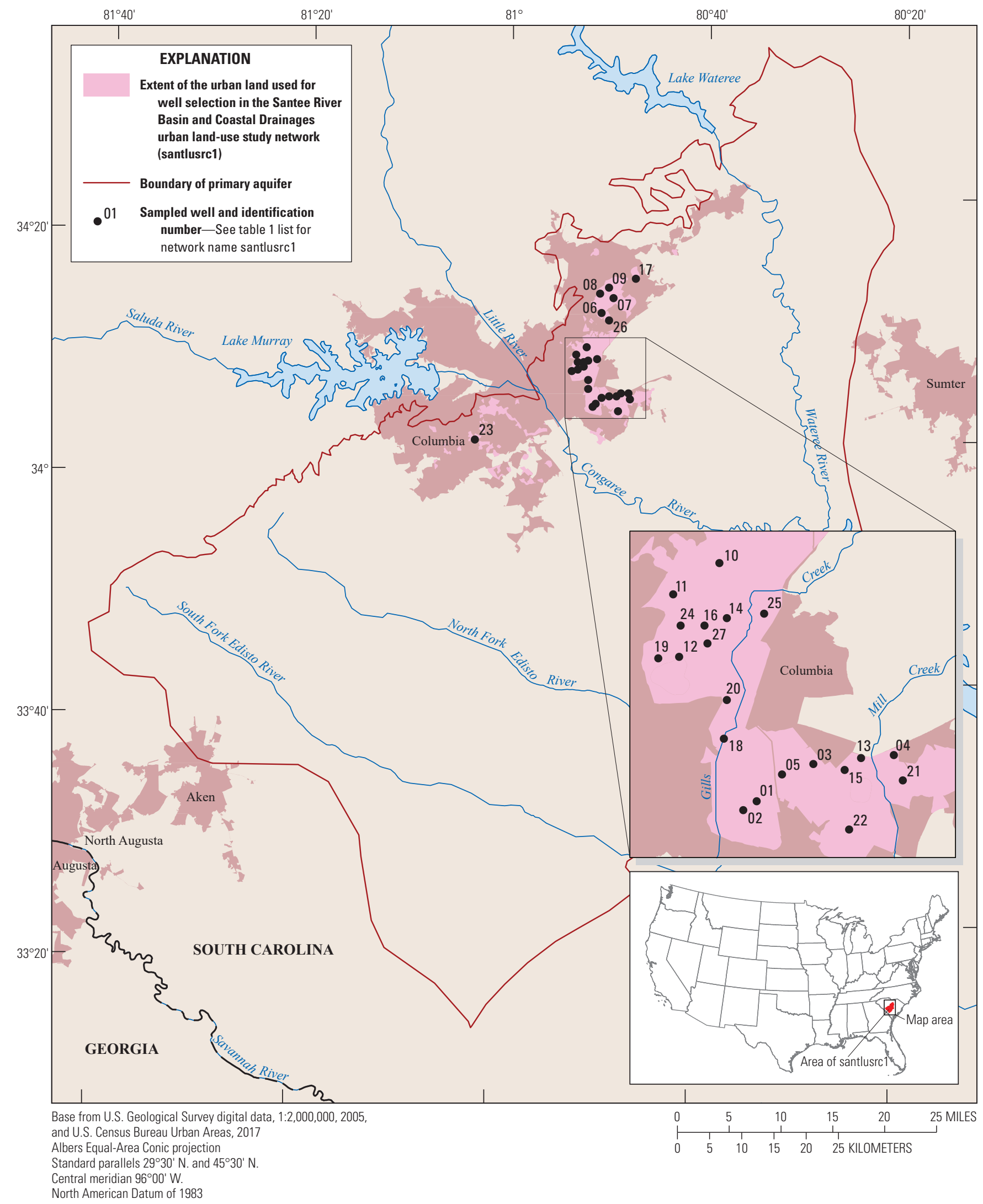

Figure 9. Study area and wells sampled as part of the Santee River Basin and Coastal Drainages urban land-use study network (santusrc1) near Columbia, South Carolina, for the U.S. Geological Survey National Water-Quality Assessment Project. 


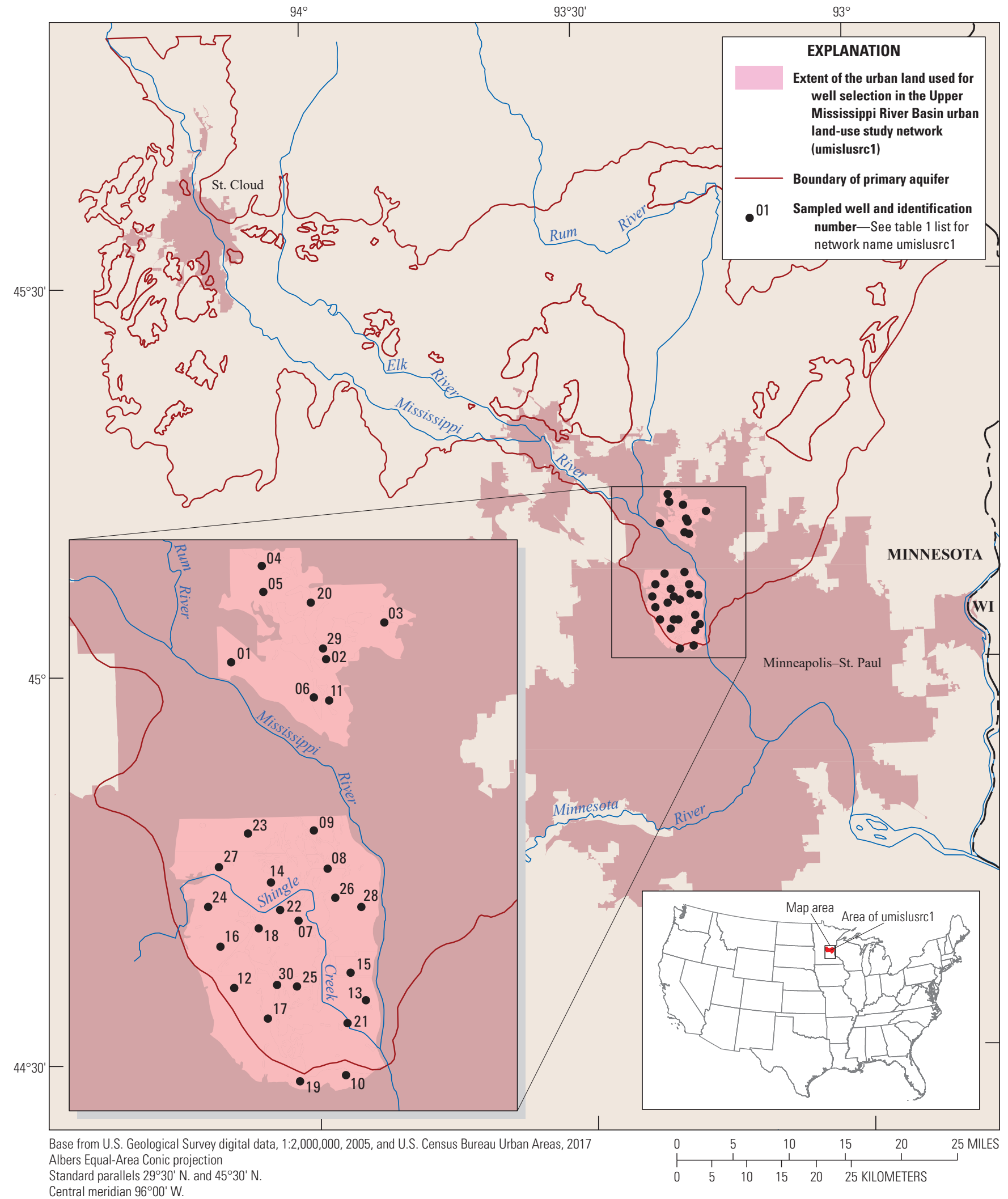

Central meridian $96^{\circ} 00^{\prime} \mathrm{W}$.

Figure 10. Study area and wells sampled as part of the Upper Mississippi River Basin urban land-use study network (umislusrc1) near Minneapolis/St. Paul, Minnesota, for the U.S. Geological Survey National Water-Quality Assessment Project. 


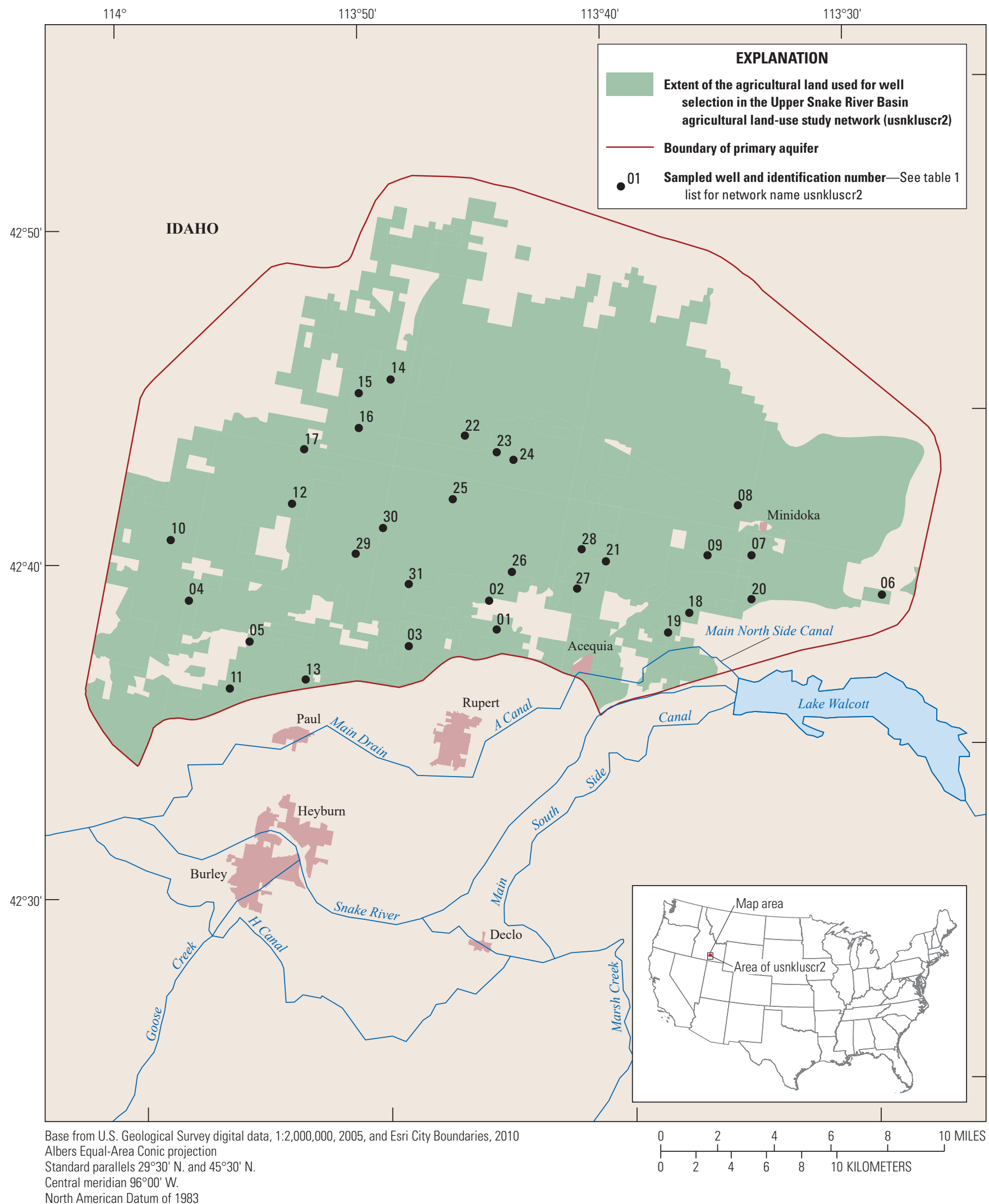

Figure 11. Study area and wells sampled as part of the Upper Snake River Basin agricultural land-use study network (usnkluscr2) for the U.S. Geological Survey National Water-Quality Assessment Project. 
The LUS sampling network consists of 28 monitoring wells. The wells were typically about 16 to $46 \mathrm{ft}$ deep (appendix 2, table 2.1) with about 4 to $8 \mathrm{ft}$ screen intervals (appendix 2, table 2.2). The same 28 wells were also sampled as part of the glacvfps1 network (table 1) described later in this report. A total of 30 monitoring wells in the network were previously sampled in 1996 or 1997 (Thomas, 2000b) and in 2006. Subsets of wells also were sampled periodically from 2002 through 2010. Samples for the current phase of monitoring were collected from August through September 2016 and are reported here.

\section{Rio Grande Aquifer System Agricultural Land-Use Study Network (rioglusag1)}

The Rio Grande Valley agricultural LUS network (rioglusag1; fig. 7) was designed to characterize shallow groundwater quality in an agricultural area overlying the Rio Grande aquifer system in southern New Mexico. The study area of about $57 \mathrm{mi}^{2}$ lies within the flood plain of the Rio Grande in the narrow Rincon Valley. The aquifer system in the area consists of nonglacial sediments that can be divided into finegrained basin-fill deposits of Tertiary age overlain by coarser valley-fill deposits of Quaternary age (Anderholm, 2002).

The rioglusag1 network consists of 24 monitoring wells completed near the water table in the Quaternary valleyfill deposits. The wells are of similar depths, ranging from about 15 to $37 \mathrm{ft}$, with a median of about $23 \mathrm{ft}$ (appendix 2 , table 2.1). The wells have open intervals of around $10 \mathrm{ft}$ (appendix 2, table 2.2). The rioglusag1 network was previously sampled in 1994 and 2006; however, because of generally declining water levels, several wells in the original 1994 network have since gone dry. Samples for the current phase of monitoring were collected in April and May of 2016. One well in this network was also sampled as part of the Rio Grande aquifer system ETN (rgaqetn1) network (table 1) described later in this report.

\section{Rio Grande aquifer system urban land-use study network (rioglusrc1)}

The Rio Grande Basin urban LUS network (rioglusrc1, fig. 8) was designed to characterize shallow groundwater quality in an urban area overlying the Rio Grande aquifer system in central New Mexico. The study area of about $190 \mathrm{mi}^{2}$ in and near Albuquerque lies within the flood plain of the Rio Grande in the alluvial Middle Rio Grande Basin. The aquifer system in the basin consists of nonglacial sand and gravel that can be divided into basin-fill deposits of Tertiary age, generally thousands of feet thick, overlain by valley-fill deposits of Quaternary age, typically less than about $130 \mathrm{ft}$ thick (Hawley and Haase, 1992). The aquifer system is used mainly for municipal and domestic water supply. Although the network is intended to focus on water quality underlying areas of residential and commercial land use, the study area also includes agricultural land (Anderholm, 1997).

The rioglusrc1 network consists of 27 monitoring wells completed near the water table in the Quaternary valley-fill deposits. The wells range in depth from about 13 to $57 \mathrm{ft}$, with a median of about $27 \mathrm{ft}$ (appendix 2, table 2.1). Open intervals range from 5 to $15 \mathrm{ft}$ and are typically about $10 \mathrm{ft}$ (appendix 2, table 2.2). Several wells in the rioglusrc1 network were previously sampled in 1993, 2006, or both. There were 15 wells in this network sampled also as part of the Rio Grande aquifer system VFPS (rgaqvfps1) network (table 1) described later in this report. Samples for the current phase of monitoring were collected in July and August 2016.

\section{Santee River Basin and Coastal Drainages Urban Land-Use Study Network (santusrc1)}

The Santee River Basin and Coastal drainages urban LUS network (santlusrc1; fig. 9) consists of 27 monitoring wells installed between 8 and $54 \mathrm{ft}$ deep (appendix 2, table 2.1) in semiconsolidated sand. The study area of about $3,400 \mathrm{mi}^{2}$ is near Columbia, South Carolina, and was designed to characterize shallow groundwater quality in a commercial and residential area. The wells are screened in the Middendorf Formation, which is part of the sandhills aquifers (Hughes and others, 2000) and the McQueen Branch Aquifer (Campbell and Landmeyer, 2014). This network previously was sampled in 1996 and 2006. Samples for the current phase of monitoring were collected in August 2016.

\section{Upper Mississippi River Basin Urban Land-Use Study Network (umislusrc1)}

The Upper Mississippi River Basin urban LUS network (umislusrc1; fig. 10) was designed to characterize the effects of urban residential and commercial development activities on shallow groundwater in a surficial sand and gravel aquifer system in the northwestern part of Minneapolis-St. Paul, Minnesota. The study area covers about 1,600 $\mathrm{mi}^{2}$. and overlies an area of 50 to greater than $400 \mathrm{ft}$ of sand, gravel, and clay, which were deposited as river terraces or overbank deposits by melt water from the Des Moines Lobe during the late Wisconsinin glacial period (Andrews and others 1997). Estimated transport times from the land surface to the water table indicate that this sand and gravel aquifer is highly susceptible to contamination from activities at the land surface (Peigat, 1989; Meyer, 1993).

The umislusrc1 consists of 30 shallow monitoring wells completed immediately below the water table at depths from about 9 to $33 \mathrm{ft}$ with a median of $18 \mathrm{ft}$, (appendix 2, table 2.1). Open intervals generally were $5 \mathrm{ft}$ (appendix 2, table 2.2). Wells from the umislusrc1 network were previously 
sampled in 1997-98 and 2006. Samples for the current phase of monitoring were collected during July, August, and September 2016, and these data are presented in this report.

\section{Upper Snake River Basin Agricultural Land-Use Study Network (usnkluscr2)}

The Upper Snake River Basin agricultural LUS network (usnkluscr2; fig. 11), with a study area of about $400 \mathrm{mi}^{2}$, was designed to characterize groundwater quality underlying agricultural land use in the East Snake River Plain aquifer in the Upper Snake River Basin in southern Idaho. The aquifer is composed of a series of Quaternary basalt flows that are vesicular and broken and able to transmit large volumes of water (Whitehead, 1992). The study area is characterized by a history of elevated nitrate concentrations in groundwater and is predominantly an area of groundwater-sourced irrigated agriculture (Rupert, 1997; Skinner, 2003).

The usnkluscr2 network includes 31 wells, mostly used for domestic supply. The wells range in depth from 125 to $469 \mathrm{ft}$ (median of $256 \mathrm{ft}$ ) with a large range of open intervals from 2 to about $337 \mathrm{ft}$ (median of $171 \mathrm{ft}$; appendix 2; table 2.1). Most wells are cased at the surface only with open-hole well construction. Samples for the current phase of monitoring were collected during June 2016.

\section{Decadal Trends Networks-Major Aquifer Study Networks}

The MAS networks were designed to reflect the resource used for domestic supply. The MAS networks generally consist of domestic-supply wells but also may include publicsupply or other types of wells. Domestic-supply wells typically draw smaller volumes of groundwater and from shallower depths of the aquifer than do public-supply wells. The MAS areas are determined by the areal extent of the primary aquifer and physiography and are designed to assess the condition of groundwater quality in the most heavily used aquifer in the area (Lapham and others, 1995; Koterba and others, 1995). Wells in MAS networks are sampled once per decade to assess temporal trends in water quality. Data from the following MAS networks are included in this report: Lake Erie-Lake St. Clair drainages MAS network (lerisus1; fig. 12), Nevada Basin and Range MAS network (nvbrsus2; fig. 13), and Puget Sound drainages MAS network (pugtsus1; fig. 14).

\section{Lake Erie-Lake St. Clair Drainages Major Aquifer Study Network (lerisus1)}

The Lake Erie-Lake St. Clair drainages MAS network (lerisus1; fig. 12) was established to assess the water quality of an aquifer that is an important source of drinking water in the developed area north and west of Detroit, Mich. The lerisus 1 study area covers about 2,500 $\mathrm{mi}^{2}$ and coincides with the boundary of the lerilusrc1 network (described previously). Groundwater is drawn from discontinuous layers of sand and gravel that are present at various depths within the glacial till. Collectively, these layers of sand and gravel are an aquifer system that is variably confined (Fleming, 1994). The hydrogeology is complex because of heterogeneity of glacial materials, fracturing in the surficial till, and the network of agricultural tile drains. (Thomas, 2000a).

The lerisus 1 consists of 24 domestic wells. The wells ranged from 35 to $205 \mathrm{ft}$ deep (appendix 2, table 2.1) with 3 - to 10-ft screen intervals (appendix 2, table 2.2). A total of 27 wells in the network were previously sampled in 1997 (Thomas, 2000b). Samples for the current phase of monitoring were collected from August through September 2016, and these data are reported here.

\section{Nevada Basin and Range Major Aquifer Study Network (nvbrsus2)}

The Nevada Basin and Range MAS Network (nvbrsus2; fig. 13) was designed to broadly assess the quality of groundwater in the Basin and Range basin-fill aquifers, specifically in the deep Truckee Meadows and Eagle Valley alluvial aquifers within the Truckee and Carson River Basins in western Nevada. The nvbrsus2 study area is about $380 \mathrm{mi}^{2}$ overlying an aquifer of nonglacial sand and gravel. Historically, agriculture was the chief land use in both valleys; however, most land has been urbanized with only about 1,200 acres for agriculture remaining in the Truckee Meadows and less than 500 acres in Eagle Valley (Homer and others, 2015).

The nvbrsus 2 includes 30 wells, mostly public-supply wells (table 1). The wells range from 105 to $815 \mathrm{ft}$ deep and have a median depth of $347 \mathrm{ft}$ (appendix 2, table 2.1). The wells have a wide range of open intervals from 10 to $670 \mathrm{ft}$, with a median of around $193 \mathrm{ft}$ (appendix 2, table 2.2). This network was previously sampled in1995 and 2003. Samples for the current phase of monitoring were collected in July through September 2016.

\section{Puget Sound Drainages Major Aquifer Study Network (pugtsus1)}

The Puget Sound Drainages MAS network (pugtsus1; fig. 14) was established to characterize the quality of shallow groundwater in the unconfined parts of the Fraser aquifer. The pugtsus 1 study area covers about 2,100 $\mathrm{mi}^{2}$ between the Canadian border, the Olympic Mountains, and the Cascade Range. Aquifers in the Puget Sound area are contained mostly within the Puget Sound Lowland, which is a structural basin filled with alluvial, glacial, and interglacial unconsolidated sediments, which can be locally more than $3,000 \mathrm{ft}$ thick. (Jones, 1999). These aquifers are vulnerable to contamination where they are shallow, coarse-grained, unconfined, and overlain by urban or agricultural land use (Tesoriero and Voss, 1997). The Fraser aquifer is composed primarily of 




Albers Equal-Area Conic projection

Standard parallels $29^{\circ} 30^{\prime} \mathrm{N}$. and $45^{\circ} 30^{\prime}$ N., Central meridian $96^{\circ} 00^{\prime} \mathrm{W}$.

North American Datum of 1983

Figure 12. Study area and wells sampled as part of the Lake Erie-Lake St. Clair drainages major aquifer study network (lerisus1) for the U.S. Geological Survey National Water-Quality Assessment Project. 


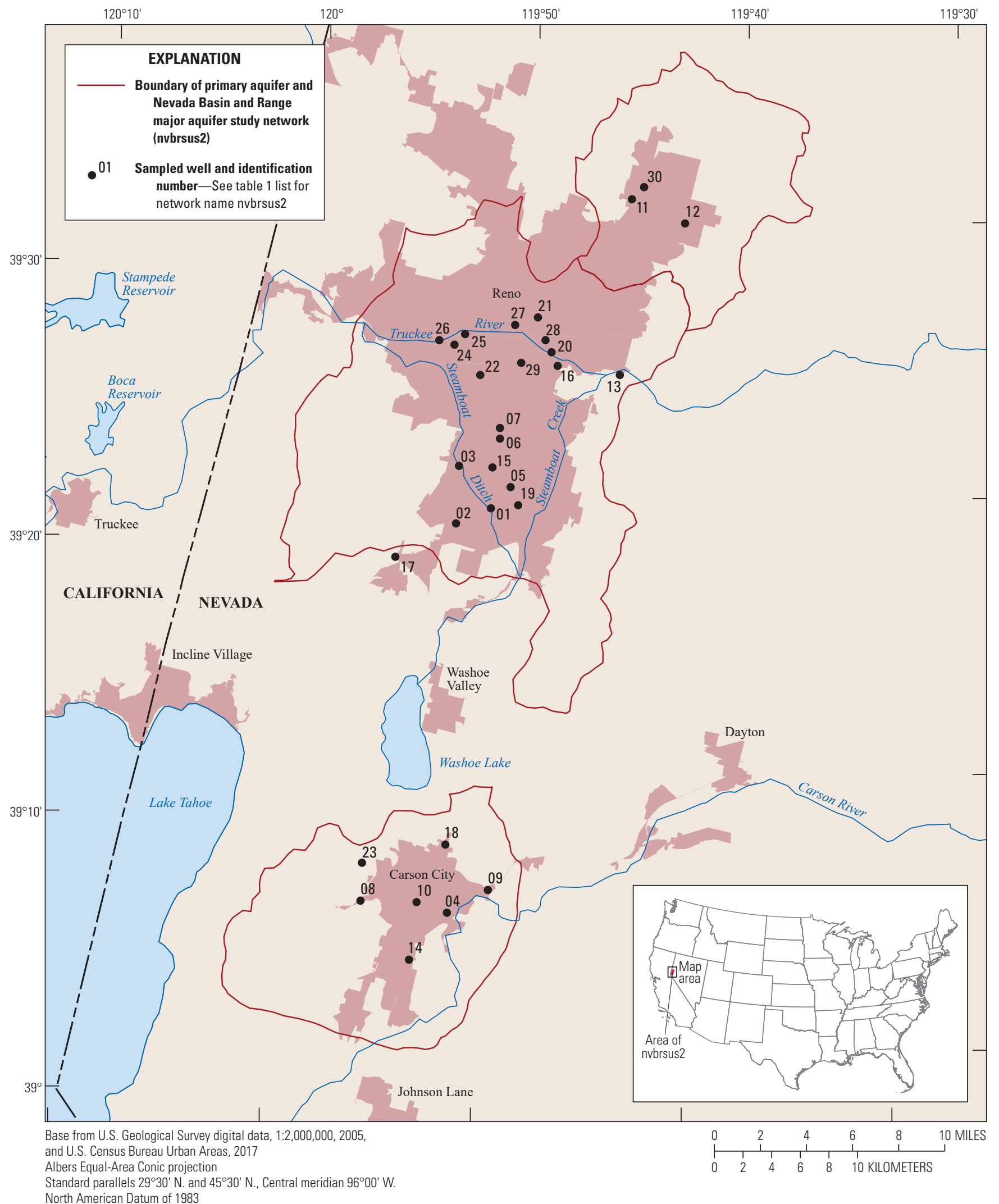

Figure 13. Study area and wells sampled as part of the Nevada Basin and Range major aquifer study network (nvbrsus2) for the U.S. Geological Survey National Water-Quality Assessment Project. 


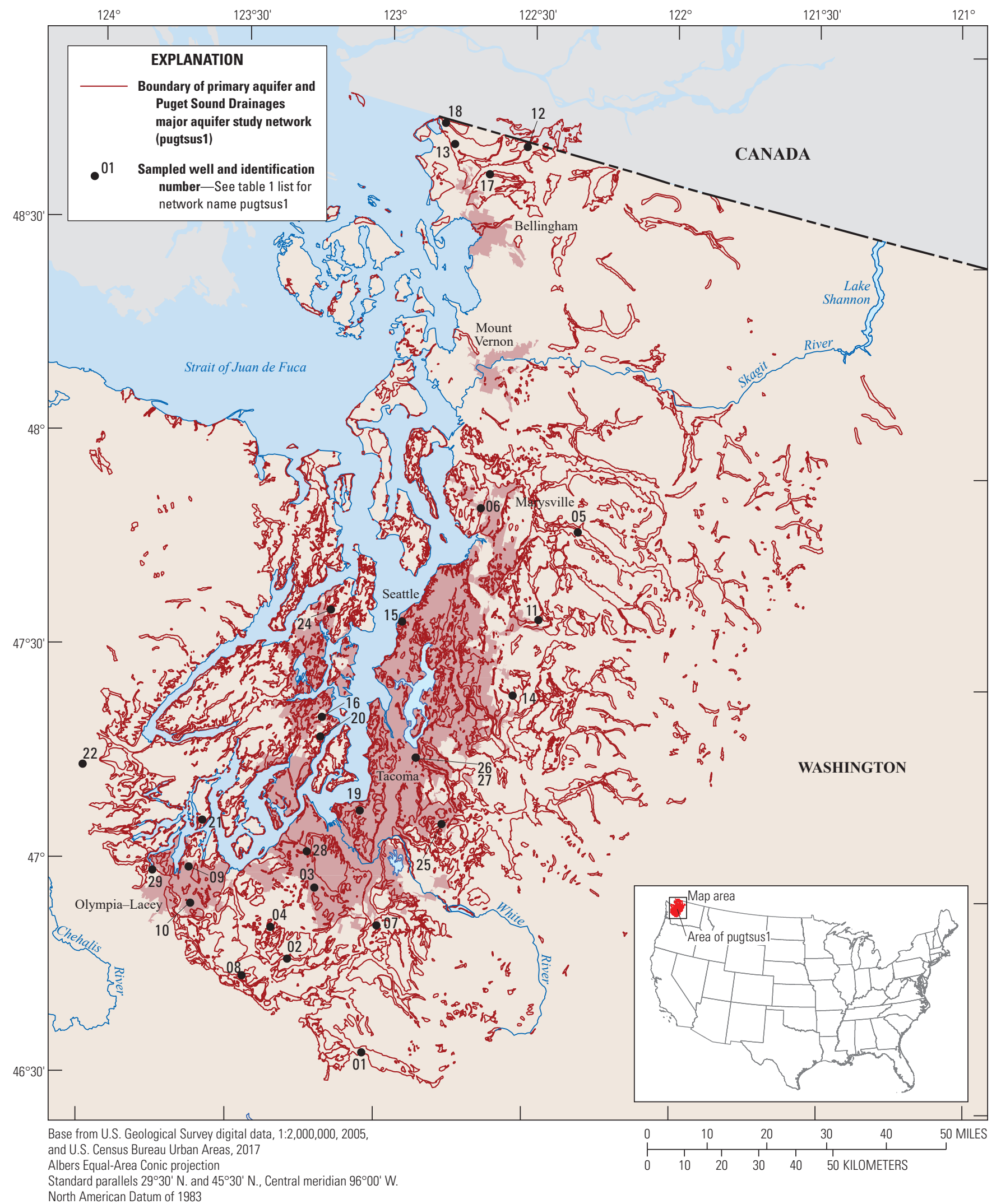

Figure 14. Study area and wells sampled as part of the Puget Sound drainages major aquifer study network (pugtsus1) for the U.S. Geological Survey National Water-Quality Assessment Project. 
coarse-grained outwash deposited during glacial advances and retreats, with some finer materials interbedded (Jones, 1999). The quality of groundwater of the Puget Sound Basin area is of concern because of its importance for municipal and domestic supplies of potable water (Staubitz and others, 1997).

The pugtsus 1 consists of 29 primarily drinking water wells (table 1). The wells were between about 22 and $309 \mathrm{ft}$ deep (appendix 2, table 2.1) with screens generally between 5 and $10 \mathrm{ft}$ long but as much as $60 \mathrm{ft}$ long (appendix 2, table 2.2). A total of 30 wells in the network were previously sampled in 1996 (Inkpen and others, 2000). Samples for the current phase of monitoring were collected in August through December 2016, and these data are reported here.

\section{Enhanced Trends Networks}

An ETN consists of a small number of wells (typically two to four) that are sampled frequently to evaluate the time scales during which groundwater quality changes. Such changes might result from seasonal or annual variability in recharge, discharge, or contaminant loading (Rowe and others, 2013). Data from eight ETNs are included in this report (figs. 15-16): Central Valley ETN (cvaletn1), Columbia Plateau ETN (clptetn1), Edwards-Trinity aquifer system ETN (edtretn1), glacial aquifer system ETN (glacetn1), Mississippi Embayment aquifer system ETN (metxent1), Northern Atlantic Coastal Plain ETN (nacpetn1), New England crystalline-rock and glacial aquifer system ETN (negxetn1), and the Rio Grande aquifer system ETN (rgaqetn1).

Wells in an ETN are instrumented for high-frequency measurement of selected parameters and they periodically have discrete measurements of additional parameters. The parameters measured at a high frequency differ among wells and networks but generally include parameters like temperature, dissolved oxygen, $\mathrm{pH}$, and specific conductance. Data collected at a high frequency for wells in the ETNs are available online; links to the data are provided in appendix 3, table 3.1.

For periodic discrete sampling, the ETNs are divided into two groups of four networks that are sampled on a 4-year alternating cycle. Four networks are sampled about once every 2 months for 4 years, whereas the other four networks are sampled annually. After the first 4-year period, the sampling frequency switches; the networks that were sampled every 2 months during the first period are sampled annually, and the other four networks are sampled every 2 months. Water-quality data from the discrete sampling during 2016 are included in this report and in Arnold and others (2020).

\section{Central Valley Enhanced Trends Network (cvaletn1)}

The Central Valley ETN (cvaletn1; fig. 15A, C) in the Central Valley aquifer system is intended to aid in the understanding of the subsurface movement of groundwater constituents (in some cases contaminants from land-use practices) between the shallow and deep parts of the aquifer system. The environmental setting of cvaletn 1 previously was described in Arnold and others (2017a,b) and is not repeated in this report.

The cvaletn 1 is made up of three wells that represent different depths in the regional aquifer (table 1). Two wells are relatively shallow (CVALETN1-02, $320 \mathrm{ft}$ deep; and CVALETN1-03, $234 \mathrm{ft}$ deep), and one well is relatively deep (CVALETN1-01, $620 \mathrm{ft}$ deep). All three wells in the cvaletn1 were sampled previously in 2013 (Arnold and others, 2016a,b), 2014 (Arnold and others, 2017a,b), and 2015 (Arnold and others, 2018a,b). CVALETN1-02 also was sampled in 2013 as part of the cvalfps 2 network (table 1) described later in this report. Data from the 2016 sampling are included in this report and in Arnold and others (2020).

\section{Columbia Plateau Enhanced Trends Network (clptetn1)}

The Columbia Plateau ETN (clptetn1; fig. 15A, B) in the Columbia Plateau aquifer system was designed to investigate questions about how groundwater quality differs between the shallow basin-fill (unconsolidated deposits) aquifers and the deeper, underlying basaltic-rock aquifers and how water quality varies along the flow paths. The environmental setting of clptetn 1 previously was described in Arnold and others $(2017 \mathrm{a}, \mathrm{b})$ and is not repeated in this report.

The clptetn1 is made up of wells that represent different positions within the regional groundwater flow system at different depths (table 1). Well CLPTETN1-01 is a shallow (80 ft), domestic well in the sand and gravel aquifer. Well CLPTETN1-04 is a deep $(1,116 \mathrm{ft})$, long-screened (926-1,100 ft) supply well that is open to the basaltic-rock aquifers; this well is about $20 \mathrm{mi}$ south of the Columbia River and is the most proximal of the three wells. Well CLPTETN1-05 is a shallow to moderate depth (170 ft) industrial well, cased to $144 \mathrm{ft}$ also in the sand and gravel aquifer.

Wells CLPTETN1-02 and CLPTETN1-03 were sampled as part of the clptetn1 in 2014 (Arnold and others, 2017a,b); however, because of issues with sample quality and sampling access, these wells are no longer included in the clptetn1. Well CLPTETN1-01 also was sampled in 2014 (Arnold and others, 2017a,b). Wells CLPTETN1-01, CLPTETN1-04, and CLPTETN1-06 were sampled in 2015, and the data were reported in Arnold and others (2018a,b). Well CLPTETN1-06 was not resampled in 2016 because access to the well was denied; well CLPTETN1-05 was sampled instead. CLPTETN1-04 was also sampled as part of the clptpas1 network (table 1) described earlier in this report. Wells CLPTETN1-01, CLPTETN1-04, and CLPTETN1-05 were sampled in 2016 and the data are included in this report and in Arnold and others (2020). 


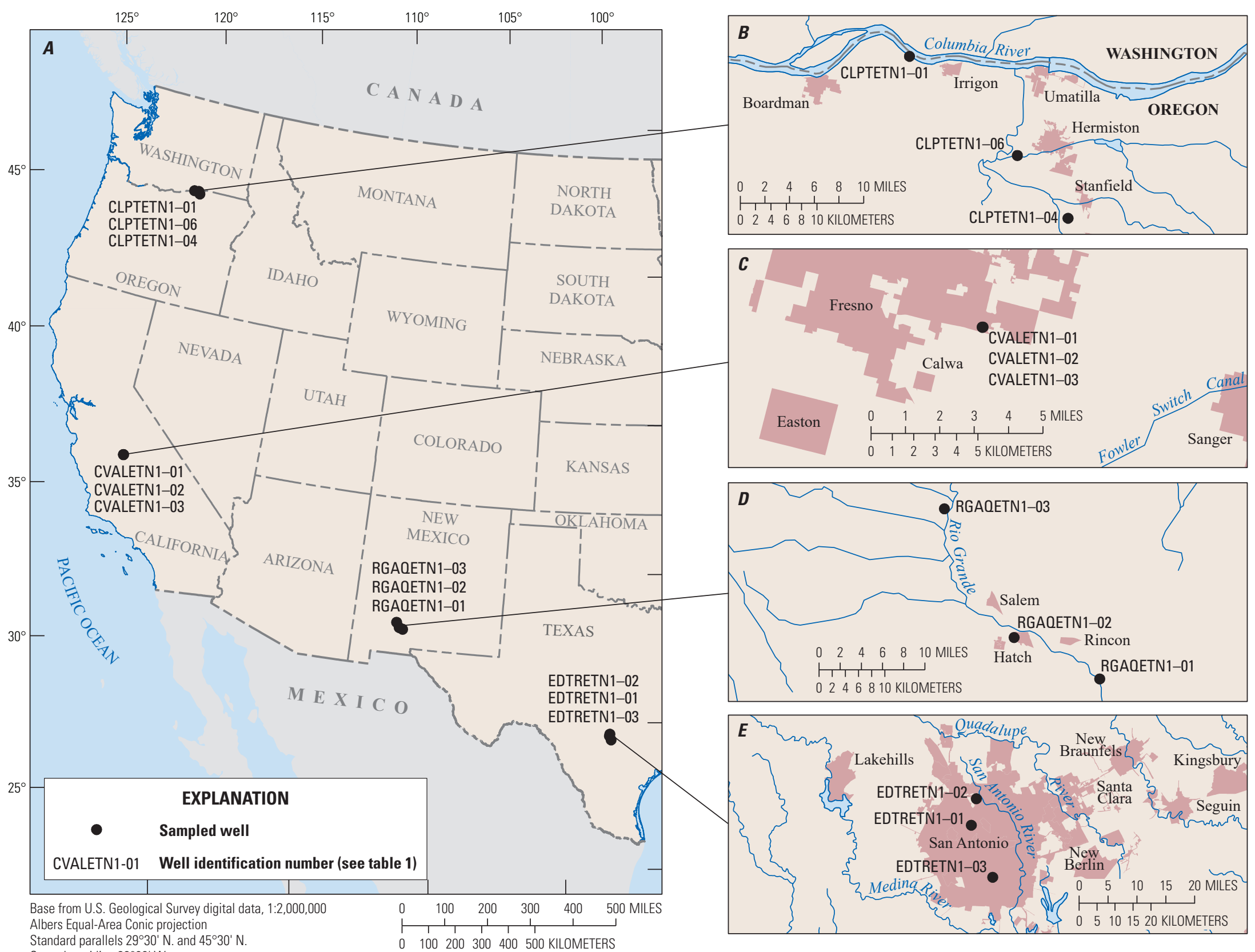

Figure 15. The, $A$, study areas and wells sampled in the western United States as part of the enhanced trends networks for the U.S. Geological Survey National Water-Quality Assessment Project; and details of well locations for, $B$, clptetn1; $C$, cvaletn1; $D$, rgaqetn1; and, $E$, edtretn1. 

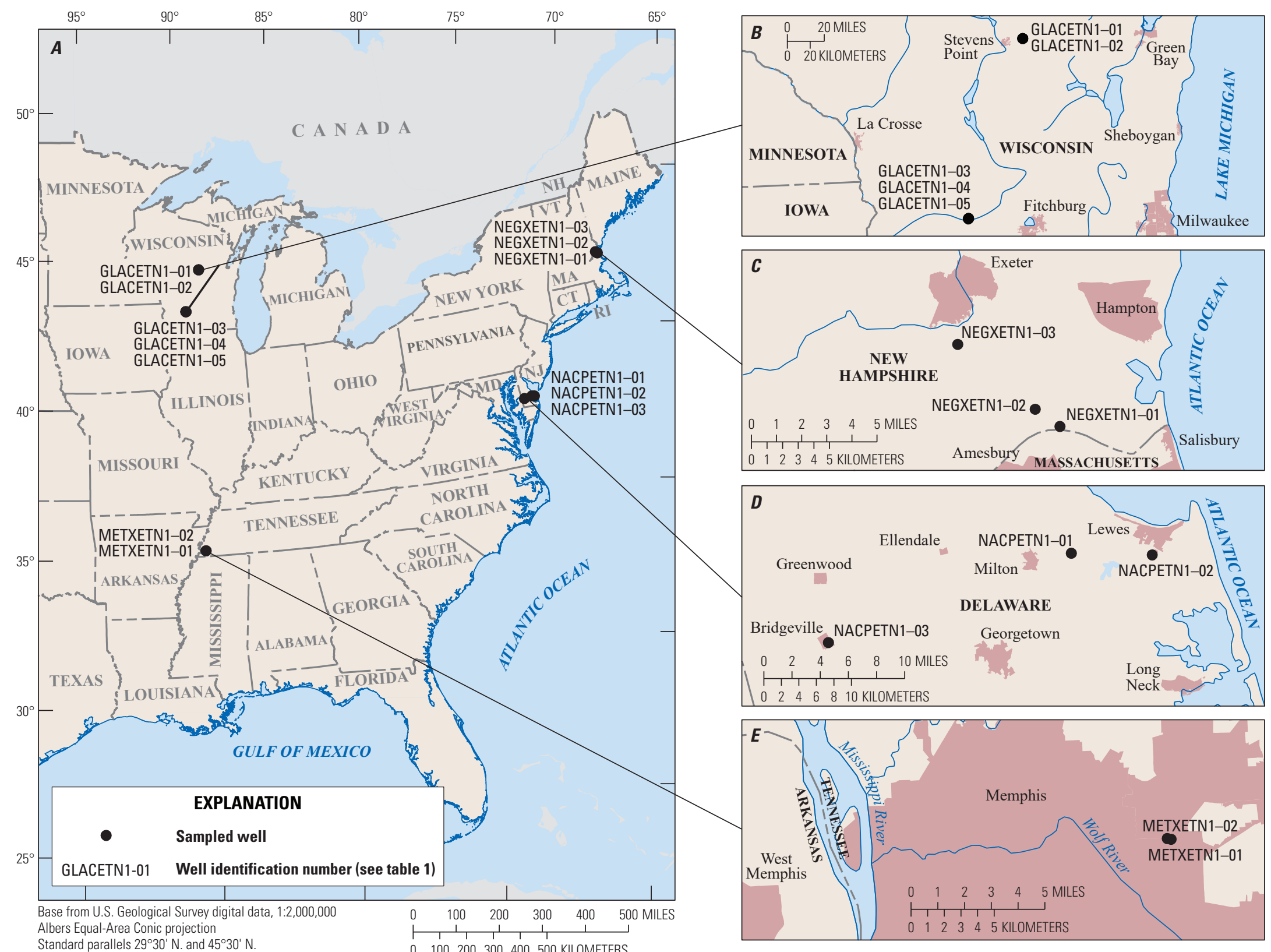

Standard parallels $29^{\circ} 30^{\prime} \mathrm{N}$. and 45 $30^{\circ} \mathrm{N}$.

Central meridian $96^{\circ} 00^{\prime} \mathrm{W}$.

North American Datum of 1983

Figure 16. The, $A$, study areas and wells sampled in the eastern United States as part of the enhanced trends networks for the U.S. Geological Survey National Water-Quality Assessment Project; and details of well locations for, $B$, glacetn1; $C$, negxetn1; $D$, nacpetn1; and, $E$, metxetn1. 


\section{Edwards-Trinity Aquifer System Enhanced Trends Network (edtretn1)}

The Edwards-Trinity aquifer system ETN (edtretn1; fig. $15 \mathrm{~A}, \mathrm{E}$ ) was designed to evaluate temporal variability in groundwater quality in a dynamic karst aquifer. The environmental setting of edtretn 1 previously was described in Arnold and others $(2017 \mathrm{a}, \mathrm{b})$ and is not repeated in this report.

There are three wells in the edtretn 1 (table 1) that are along an approximately north-to-south aquifer transect within the San Antonio metropolitan area (fig. 15E). One well is in the upgradient, unconfined recharge zone, and two wells are downgradient in the confined zone. The upgradient well (EDTRETN1-02) is $300 \mathrm{ft}$ deep and open to the aquifer along the bottom $80 \mathrm{ft}$ of its depth (table 1). The downgradient wells are $550 \mathrm{ft}$ (EDTRETN1-01) and 1,550 ft (EDTRETN1-03) deep and are open to the aquifer throughout their length below the confined zone (table 1). The farthest downgradient well (EDTRETN1-03) is close to the southern boundary of the aquifer. Wells in the edtretn1 were first sampled as part of the edtretn 1 in 2013 (Arnold and others, 2016a,b), and were sampled again in 2014 (Arnold and others, 2017a,b) and 2015 (Arnold and others, 2018a,b). During 2016, the wells were sampled about bimonthly from January through October 2016, and these data are included in this report and in Arnold and others (2020).

\section{Glacial Aquifer System Enhanced Trends Network (glacetn1)}

The glacial aquifer system ETN (glacetn1; fig. 16A, B) was designed to identify the temporal variability and magnitude of observed changes in groundwater quality in agricultural areas of the glacial aquifer system. The environmental setting of glacetn 1 previously was described in Arnold and others $(2017 \mathrm{a}, \mathrm{b})$ and is not repeated in this report.

The glacetn 1 consists of five wells distributed in two locations. A location in central Wisconsin has two monitoring wells: GLACETN1-01 (83 ft deep) and GLACETN1-02 (34.5 ft deep). A location in southwestern Wisconsin has three wells: monitoring wells GLACETN1-03 (50 ft deep) and GLACETN1-04 (89 ft deep), and public-supply well GLACETN1-05 (125 ft deep). All wells in the glacetn1 were sampled in 2016, and these data are included in this report and in Arnold and others (2020). Wells GLACETN1-01 and GLACETN1-02 also were sampled in 2014 as part of the Glacial aquifer FPS (glacfps1) (table 1) described later in this report. Wells GLACETN1-01 and GLACETN1-02 have been sampled every year since 2014; and GLACETN1-03, GLACETN1-04, and GLACETN1-05 have been sampled every year since 2015 (Arnold and others, 2017a,b, 2018a,b, 2020).

\section{Mississippi Embayment Aquifer System Enhanced Trends Network (metxetn1)}

The Mississippi Embayment aquifer system ETN (metxetn1; fig. $16 A, E$ ) was designed to study how water quality in shallow and deep parts of the regional aquifer changes in response to changing hydrologic conditions and pumping. The environmental setting of metxetn 1 previously was described in Arnold and others $(2017 \mathrm{a}, \mathrm{b})$ and is not repeated in this report.

The metxetn 1 consists of one well in the shallow aquifer (METXETN1-02, $90 \mathrm{ft}$ deep) and one well in the Memphis aquifer (METXETN1-01, $624 \mathrm{ft}$ deep) (table 1). The wells were first sampled as part of the metxetn1 in 2013 (Arnold and others, 2016a,b) and again in 2014 (Arnold and others, 2017a,b) and 2015(Arnold and others, 2018a,b). In 2013, the wells also were used as part of the Mississippi Embayment aquifer system FPS (metxfps1) network (table 1) described later in this report. Sampling in 2016 was approximately bimonthly, and these data are included in this report and in Arnold and others (2020).

\section{Northern Atlantic Coastal Plain Enhanced Trends Network (nacpetn1)}

The Northern Atlantic Coastal Plain ETN (nacpetn1; fig. $16 A, D$ ) in the Northern Atlantic Coastal Plain aquifer system provides an opportunity to study the movement of contaminants from the land surface downward into aquifers and the effects of recharge and pumping on the temporal variability of water quality. The environmental setting of nacpetn1 previously was described in Arnold and others (2017a,b) and is not repeated in this report.

The nacpetn1 has three wells that are located across southern Delaware in different parts of the flow system at different depths (table 1). Well NACPETN1-03 (119 ft deep) is a public-supply well in southwestern Delaware near the center of the Delmarva Peninsula. Well NACPETN1-02 (135ft deep) also is a public-supply well, one of several supply wells for a coastal town. Well NACPETN1-01 is a shallow monitoring well (22 $\mathrm{ft})$ that is surrounded locally by agricultural land use. All three wells in the nacpetn1 were sampled once in 2014 (Arnold and others, 2017a,b), once in August 2015 (Arnold and others, 2017a,b), and approximately bi-monthly during 2016. Data from the 2016 sampling are included in this report and in Arnold and others (2020).

\section{New England Crystalline-Rock and Glacial Aquifer System Enhanced Trends Network (negxetn1)}

The New England crystalline-rock and glacial aquifer system ETN (negxetn1, fig. 16 A, C) provides the opportunity to study the temporal variability of contaminants in groundwater from geologic sources as well as contaminants from 
man-made sources with changing inputs. The environmental setting of negxetn1 previously was described in Arnold and others $(2017 \mathrm{a}, \mathrm{b})$ and is not repeated in this report.

The negxetn 1 consists of three wells at different depths (table 1). Two of the wells are public-supply wells, one completed in glacial sediments (NEGXETN1-01, $83 \mathrm{ft}$ deep) and the other one completed in the crystalline-rock aquifer (NEGXETN1-02, $492 \mathrm{ft}$ deep), and are in the southern part of the network area. The third well is a domestic-supply well in the northern part (NEGXETN1-03, about $176 \mathrm{ft}$ deep). Wells in the negxetn1 were first sampled in 2014 (Arnold and others, 2017a,b) and again in 2015 (Arnold and others, 2018a,b). The wells were sampled bimonthly January through November 2016, and these data are included in this report and in Arnold and others (2020).

\section{Rio Grande Aquifer System Enhanced Trends Network (rgaqetn1)}

The Rio Grande aquifer system ETN (rgaqetn1; fig. 15A, $D$ ) provides the opportunity to study temporal variability in the water quality of shallow groundwater affected by irrigation, river water infiltration, and variable hydrologic conditions in an arid climate. The environmental setting of rgaqetn1 previously was described in Arnold and others (2017a,b) and is not repeated in this report.

The rgaqetn 1 consists of three wells completed in the valley fill at different depths (table 1): two shallow wells that are screened across the water table (RGAQETN1-01, about $23 \mathrm{ft}$ deep; and RGAQETN1-03, $22 \mathrm{ft}$ deep) and one deeper well (RGAQETN1-02, $60 \mathrm{ft}$ deep). The wells were sampled as part of the rgaqetn1 in 2014 (Arnold and others, 2017a,b) and in 2015 (Arnold and others, 2018a,b). The wells were sampled again in 2016, and these data are included in this report and in Arnold and others (2020). One well, RGAQETN1-01 also was sampled as part of rioglusag1 in 2016 (table 1).

\section{Vertical Flow-Path Study Networks}

Vertical flow-path study (VFPS) networks are designed to evaluate changes in groundwater quality over longer periods of time than the enhanced trends networks (ETNs) and decadal trends networks (LUS and MAS networks). The wells in a VFPS network are selected from public, domestic, or monitoring wells and located such that there is a representation of wells at different depths and collocated within the area to be studied. Evaluating vertical gradients of groundwater age and contaminant concentrations facilitates the understanding of changes in groundwater quality over periods greater than 10 years. VFPS networks generally are sampled once. Data from the Columbia Plateau VFPS (clptvfps1, fig. 17), Glacial aquifer system VFPS (glacvfps1, fig. 18), and Rio Grande aquifer system VFPS (rgaqvfps1, fig. 19) are included in this report.

\section{Columbia Plateau Vertical Flow-Path Study Network (clptvfps1)}

The Columbia Plateau VFPS network (clptvfps1; fig. 17) was designed to examine changes in water quality over time and to evaluate whether agriculturally derived contaminants are moving deeper into the aquifer system. The clptvfps1 is in south-central Washington and is contained within the Columbia Basin Irrigation Project area, a large-scale irrigation project through which a series of canals divert Columbia River water to a highly productive agricultural basin. The clptvps 1 network consists of 19 wells from 2 decadal networks (Frans and others, 2012): 10 shallow monitoring wells from the Columbia Plateau agricultural land use study (ccptlusag2b; Arnold and others, 2017a,b) and 9 deeper domestic or publicsupply wells from the Columbia Plateau major aquifer study (ccptsus1b; Arnold and others, 2017a,b). The 10 agricultural land-use study wells are typically screened in unconsolidated basin-fill deposits, whereas the 9 major aquifer study wells are typically screened in underlying basaltic rocks. Well depths range from 19 to $1,000 \mathrm{ft}$ (appendix 2, table 2.1). The ccptsus $1 \mathrm{~b}$ and ccptlusag2 $\mathrm{b}$ networks were previously sampled in 1994-95 and 2002. Samples for the clptvfps1 were collected from July to September 2014.

\section{Glacial Aquifer System Vertical Flow-Path Study Network (glacvfps1)}

The Glacial aquifer system VFPS network (glacvfps1; fig. 18) was designed to add an age framework to results from decadal networks in the glacial aquifer system to help address questions of changes in water quality over time. The glacvfps 1 network is a group of 43 wells and is a combination of 28 wells from the lerilusrc 1 network described earlier in this report and 15 additional wells completed at various depths ranging from about 10 to $172 \mathrm{ft}$ (appendix 2, table 2.1). The wells were open to the aquifer across intervals of 4 to $10 \mathrm{ft}$, but most were open less than 5 -ft intervals (appendix 2, table 2.2). Samples for the vertical flow-path study were collected August through September 2016, and these data are reported here.

\section{Rio Grande Aquifer System Vertical Flow-Path Study Network (rgaqvfps1)}

The Rio Grande aquifer system VFPS (rgaqvfps1; fig. 19) is in the alluvial Middle Rio Grande Basin, lying within the Rio Grande flood plain in and near Albuquerque. The study is focused on areas of the aquifer that are recharged by seepage from the Rio Grande. High concentrations of dissolved solids, arsenic, or both have previously been observed within the study area (Plummer and others, 2004).

The rgaqvfps1 network consists of 19 monitoring wells, 15 of which are part of the rioglusrc1 urban land-use network previously described in this report (ranging in depth from 


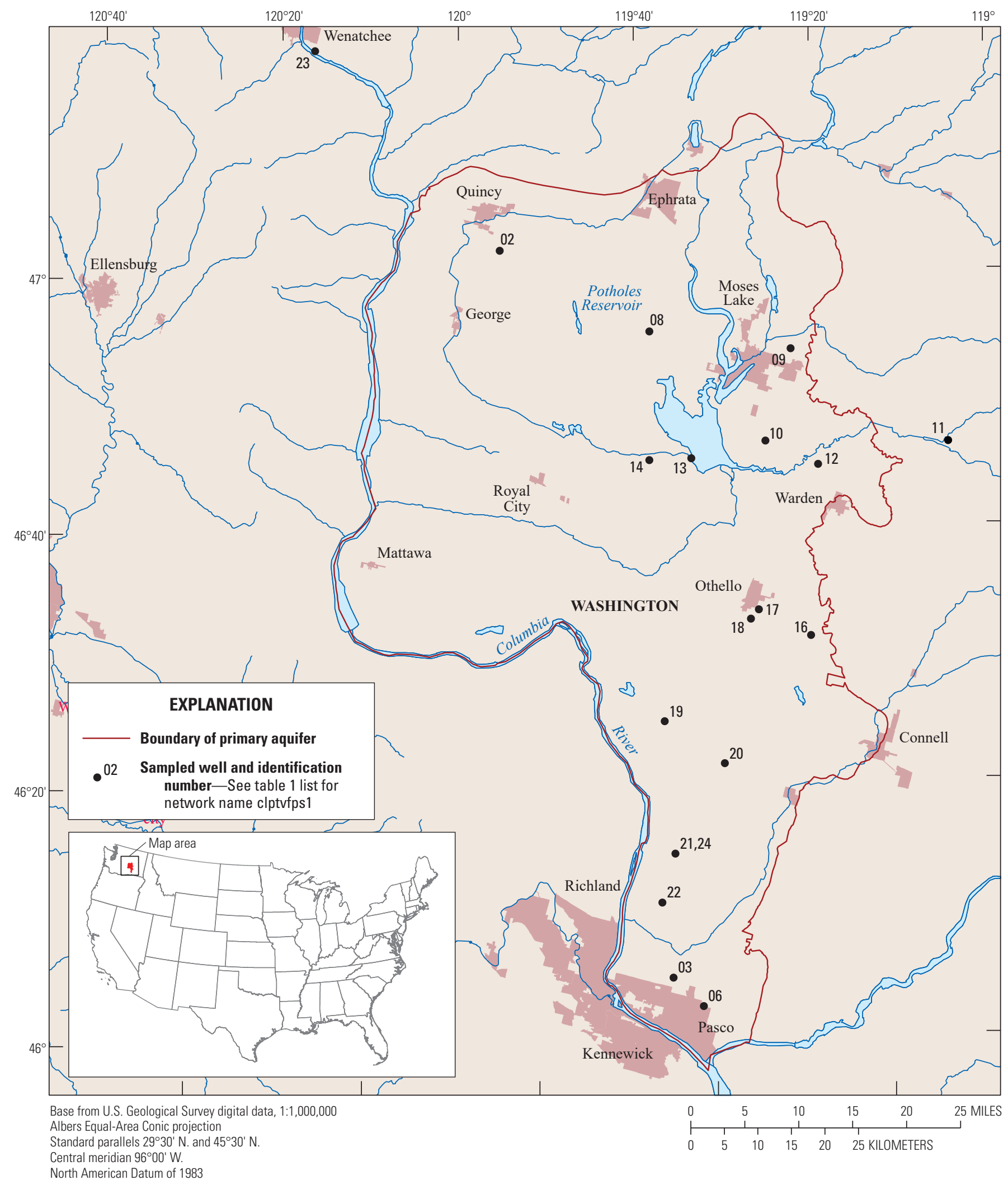

Figure 17. Study and wells sampled as part of the Columbia Plateau vertical flow-path study network (clptvfps1) for the U.S. Geological Survey National Water-Quality Assessment Project. 


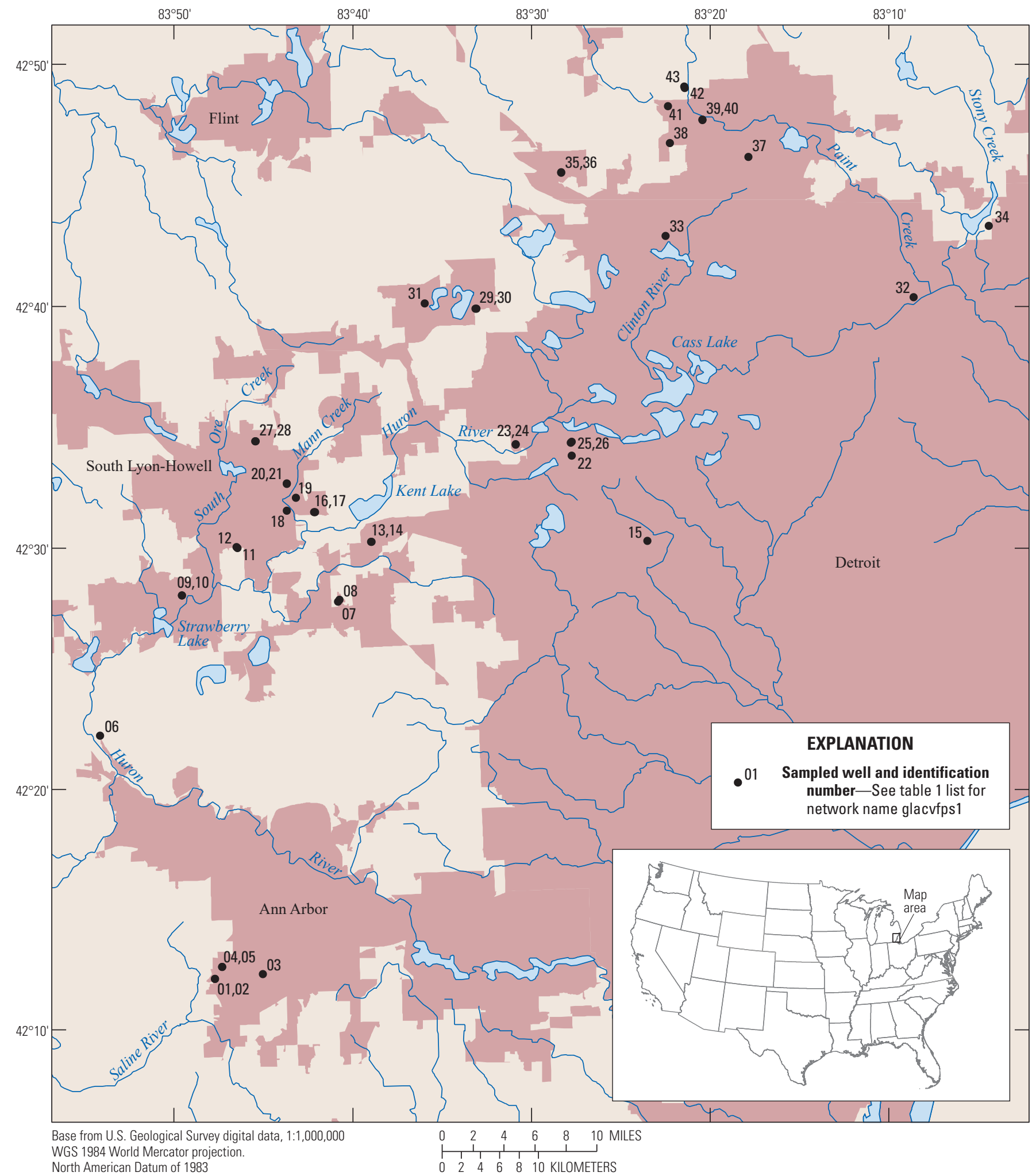

Figure 18. Study and wells sampled as part of the Glacial aquifer system vertical flow-path study network (glacvfps1) for the U.S. Geological Survey National Water-Quality Assessment Project. 


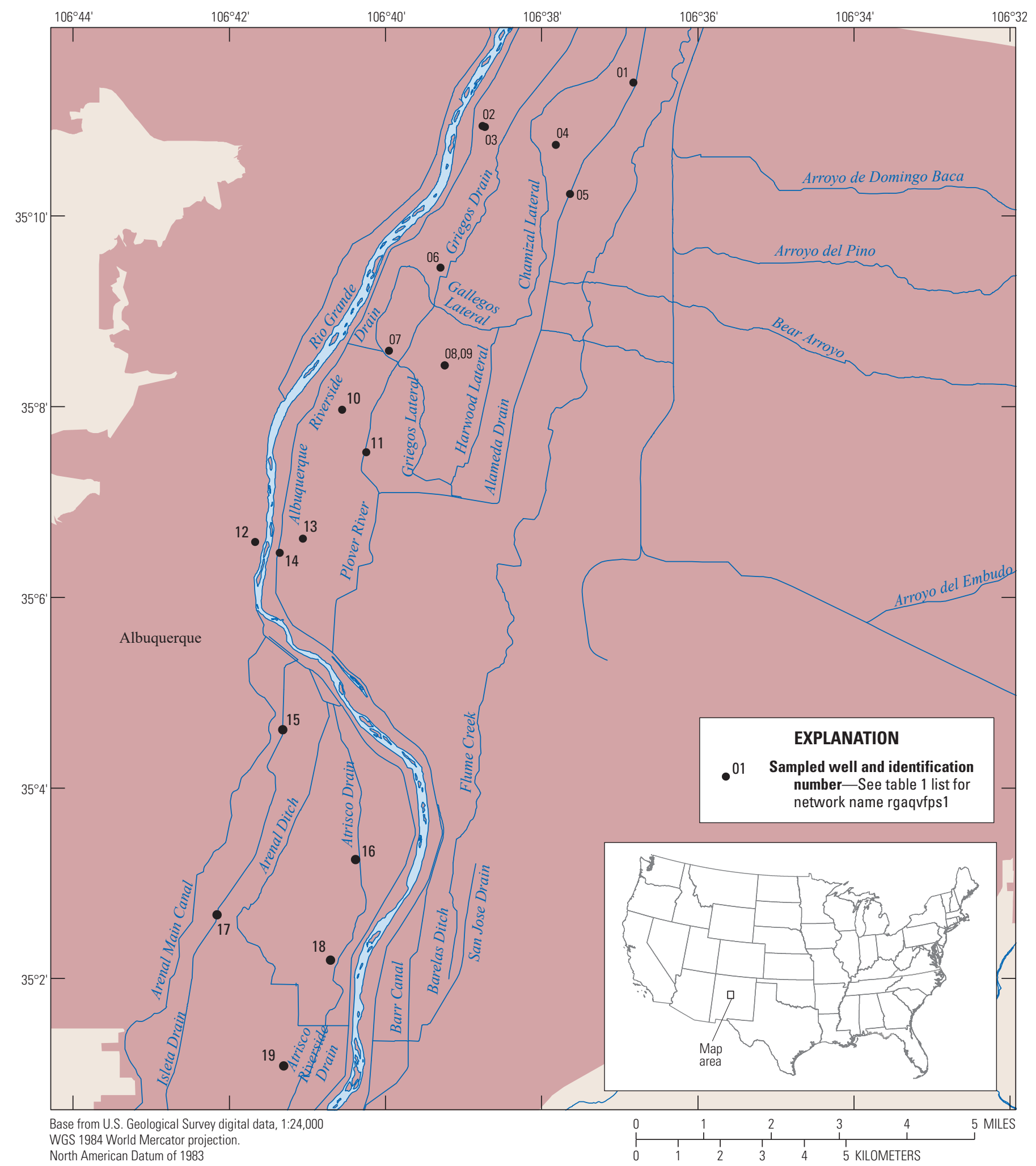

Figure 19. Study and wells sampled as part of the Rio Grande aquifer system vertical flow-path study network (rgaqvfps1) for the U.S. Geological Survey National Water-Quality Assessment Project. 
about 18 to $57 \mathrm{ft}$ ). The additional 4 wells are deeper, ranging from 95 to $254 \mathrm{ft}$ in depth (appendix 2, table 2.1). The wells are typically open to the aquifer across 10 - $\mathrm{ft}$ intervals, although intervals range from about 5 to $82 \mathrm{ft}$ (appendix 2, table 2.2). Samples were collected in July and August 2016.

\section{Flow-Path Study Networks}

Flow-path study (FPS) networks are designed to evaluate changes in groundwater quality along flow paths from recharge areas to discharges areas (streams or withdrawal from wells). For each network, monitoring wells were installed or selected at four or five locations along hypothesized groundwater flow paths, with three or four wells at each of these locations screened at various depths. These networks examine flow paths that are usually shorter (often less than about 3 miles) and shallower than VFPS networks. FPS networks are typically collocated with land-use study networks. FPS networks are designed to examine the transport and transformation of contaminants along primarily horizontal flow paths. Like the VFPS, FPS networks may be used to examine trends over decades but for a smaller spatial extent. Data from two Central Valley aquifer system FPS networks (cvalfps1 and cvalfps2; figs. 20-21); Glacial aquifer system FPS network (glacfps1; fig. 22), and Mississippi Embayment aquifer system FPS network (metxfps1; fig. 23) are included in this report.

\section{Central Valley Aquifer System Flow-Path Study Networks (cvalfps1, cvalfps2)}

The two Central Valley aquifer system FPS networks near Fresno, Calif. (cvalfps1, cvalfps2; figs. 20-21) were designed to examine the fate and transport of agricultural contaminants such as 1,2-dibromo-3-chloropropane and nitrate along groundwater flow paths. The cvalfps1 study area is west of the foothills of the Sierra Nevada and east of the San Joaquin Valley trough on the high alluvial fan of the Kings River (Burow and others, 1999). Twenty monitoring wells were installed at six well nest sites along a 2.9-mile transect to characterize changes in water quality along approximate groundwater flow paths. The monitoring well transect generally was aligned in the direction of regional groundwater movement in the study area. Well depths in the network range from 70 to $268 \mathrm{ft}$ (appendix 2; table 2.1). The cvalfps1 network was previously sampled in 1994-95 (Burow and others, 1999) and 2003 (Burow and others, 2008). Samples for the current phase of monitoring for the cvalfps1 were collected from July to September 2013.

The cvalfps 2 network is an extension in space from the cvalfps1 network using a mix of observation, irrigation, domestic-supply, and public-supply wells. There are 21 wells is the network, with well depths ranging from 100 to $532 \mathrm{ft}$ below land surface (appendix 2; table 2.1). Samples for the current phase of monitoring for the cvalfps 2 were collected from July to September 2013.

\section{Glacial Aquifer System Flow-Path Study Network (glacfps1)}

The Glacial aquifer system FPS network (glacfps1, fig. 22) was designed to examine the migration and transformation of contaminants along groundwater flow paths and groundwatersurface water interactions (Saad, 2008). The study site is in the Tomorrow River Watershed in Portage County, Wisconsin. The site includes four nests of wells installed along groundwater flow paths (as inferred from groundwater flow simulations). There are 18 total wells in these 4 nests, located upgradient from the Tomorrow River at distances of about $200 \mathrm{ft}$ (well nest 4), $700 \mathrm{ft}$ (well nest 3), 2,000 ft (well nest 2), and 5,000 ft (well nest 1). These well nests represent the downgradient part of the entire flow path length that discharges to the stream at this location. Eight additional samples were taken from streambed piezometers (tables 2.1 and 2.2 include the piezometers in the count of wells) that were about $1 \mathrm{ft}$ below the streambed. The Tomorrow River Watershed is nested within a previously conducted agricultural land-use study area (wmiclusag2, Saad, 2008; Arnold and others, 2017a,b) and is in the same location as a previous groundwater-surface water interaction study (Tesoriero and others, 2013). Samples for the current phase of monitoring for the glacfps1 were collected in June 2014.

\section{Mississippi Embayment Aquifer System Flow-Path Study Network (metxfps1)}

The Mississippi Embayment aquifer system FPS network (metxfps1; fig. 23) was designed to examine the intrinsic vulnerability of the Memphis aquifer along two inferred flow paths (Kingsbury and others, 2017). The study site is in the northeastern part of the Mississippi Embayment in southwestern Tennessee and northwestern Mississippi and is nested with a previous urban land-use study (miselusrc1). Wells are along flow paths that begin in the outcrop area of the Memphis aquifer and end at public supply wells that are in a confined part of the aquifer. This study is designed to evaluate whether younger water is migrating into confined parts of the aquifer that are used for drinking-water supplies. The network of 22 wells consists of a mix of monitoring, domestic, commercial, and public-supply wells that range in depth from 23 to $624 \mathrm{ft}$ (appendix 2; table 2.1). Samples for the current phase of monitoring for the metxfps 1 were collected in August and September 2013.

\section{Modeling Support Study Networks}

Modeling support study (MSS) networks are used to support modeling efforts where more data are needed to calibrate a model. They are designed based on a specific need so that each is somewhat unique in its design. Data from two Northern Atlantic Coastal Plain MSS networks (nacpmss1 and nacpmss2; figs. 24-25); and one Glacial aquifer system MSSS network (glacmss1; fig. 26) are included in this report. 


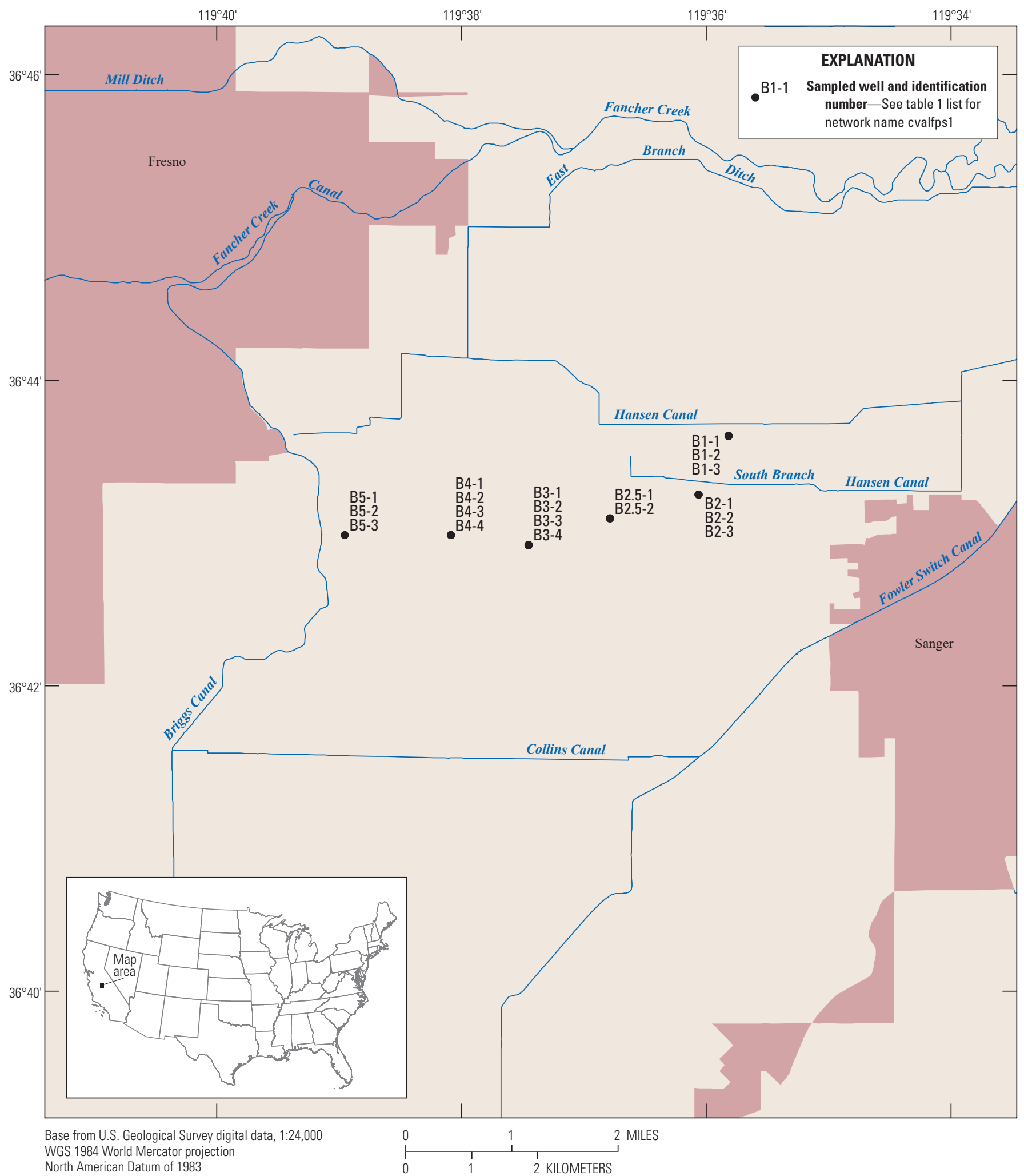

Figure 20. Study and wells sampled as part of the Central Valley aquifer system flow-path study network (cvalfps1) for the U.S. Geological Survey National Water-Quality Assessment Project. 


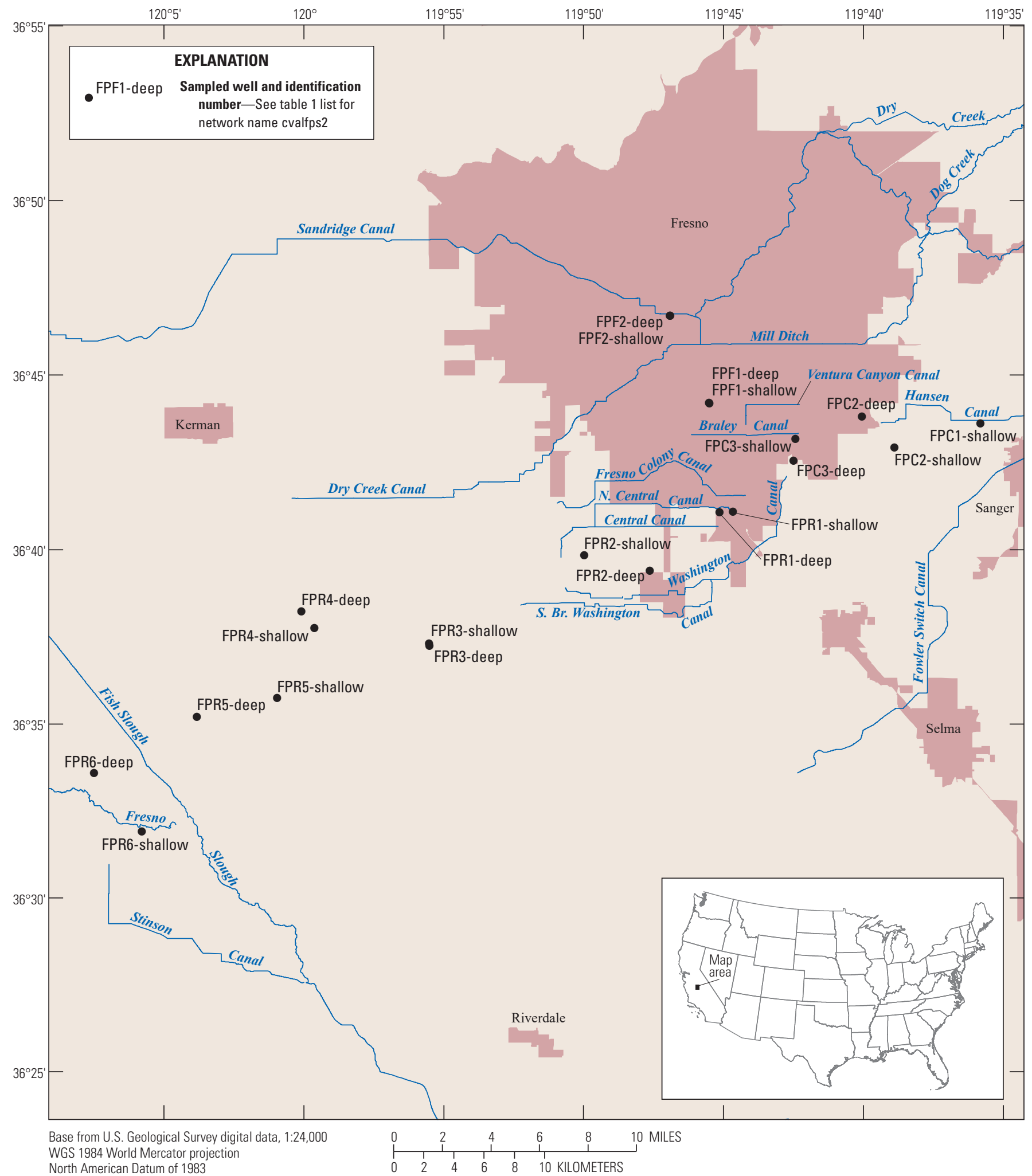

Figure 21. Study and wells sampled as part of the Central Valley aquifer system flow-path study network (cvalfps2) for the U.S. Geological Survey National Water-Quality Assessment Project. 


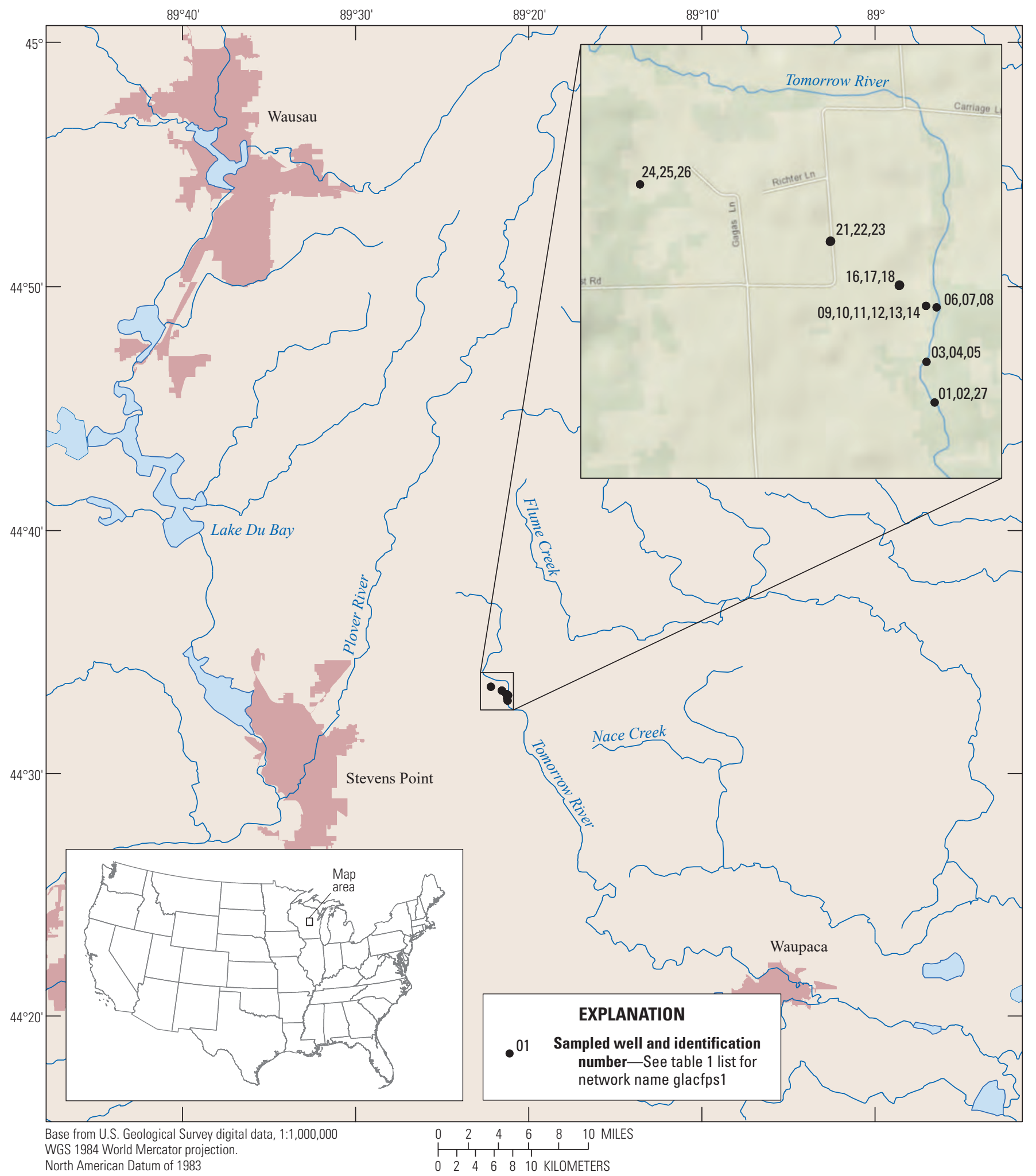

Figure 22. Study and wells sampled as part of the Glacial aquifer system flow-path study network (glacfps1) for the U.S. Geological Survey National Water-Quality Assessment Project. 


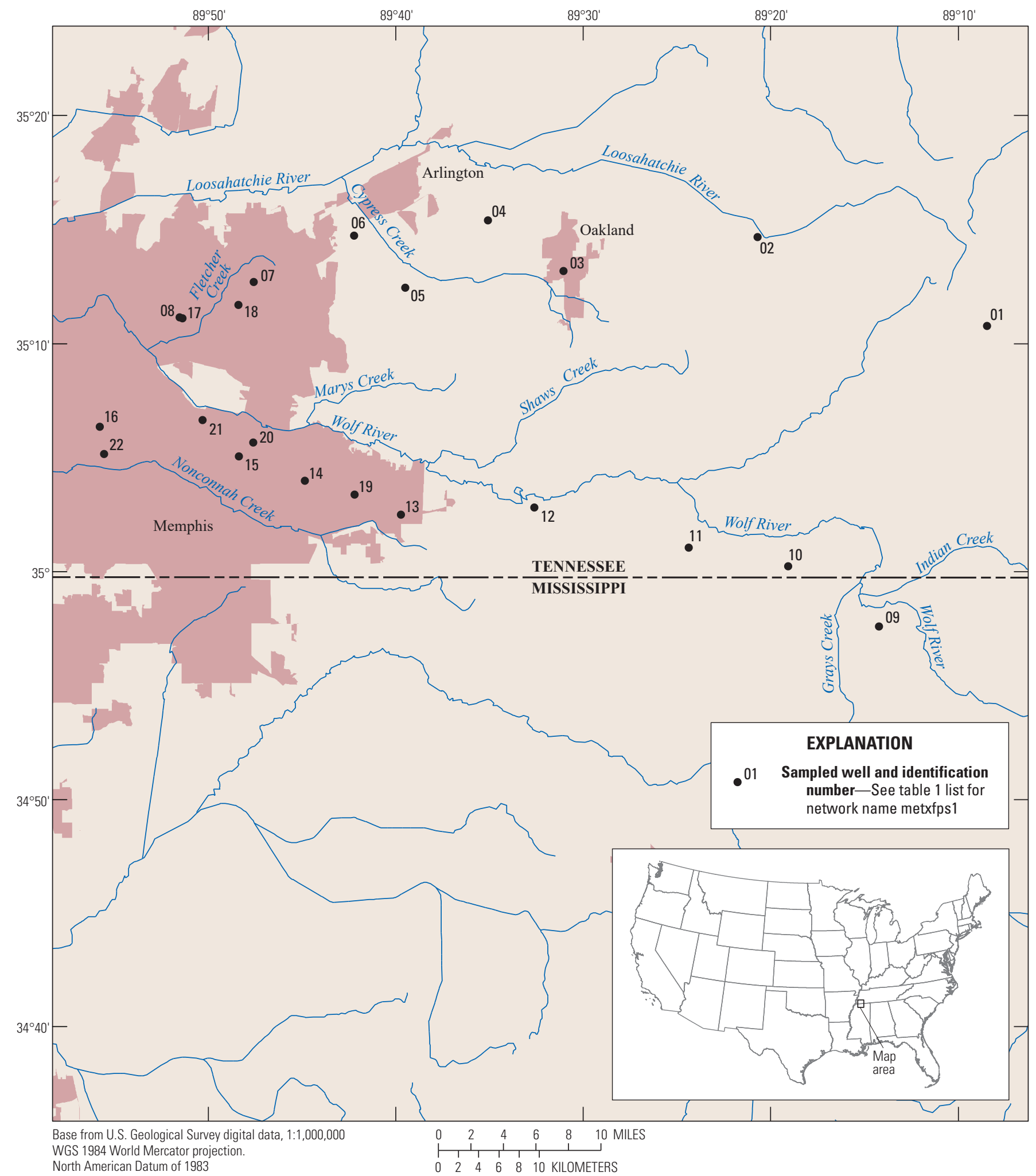

Figure 23. Study and wells sampled as part of the Mississippi Embayment aquifer system flow-path study network (metxfps1) for the U.S. Geological Survey National Water-Quality Assessment Project. 


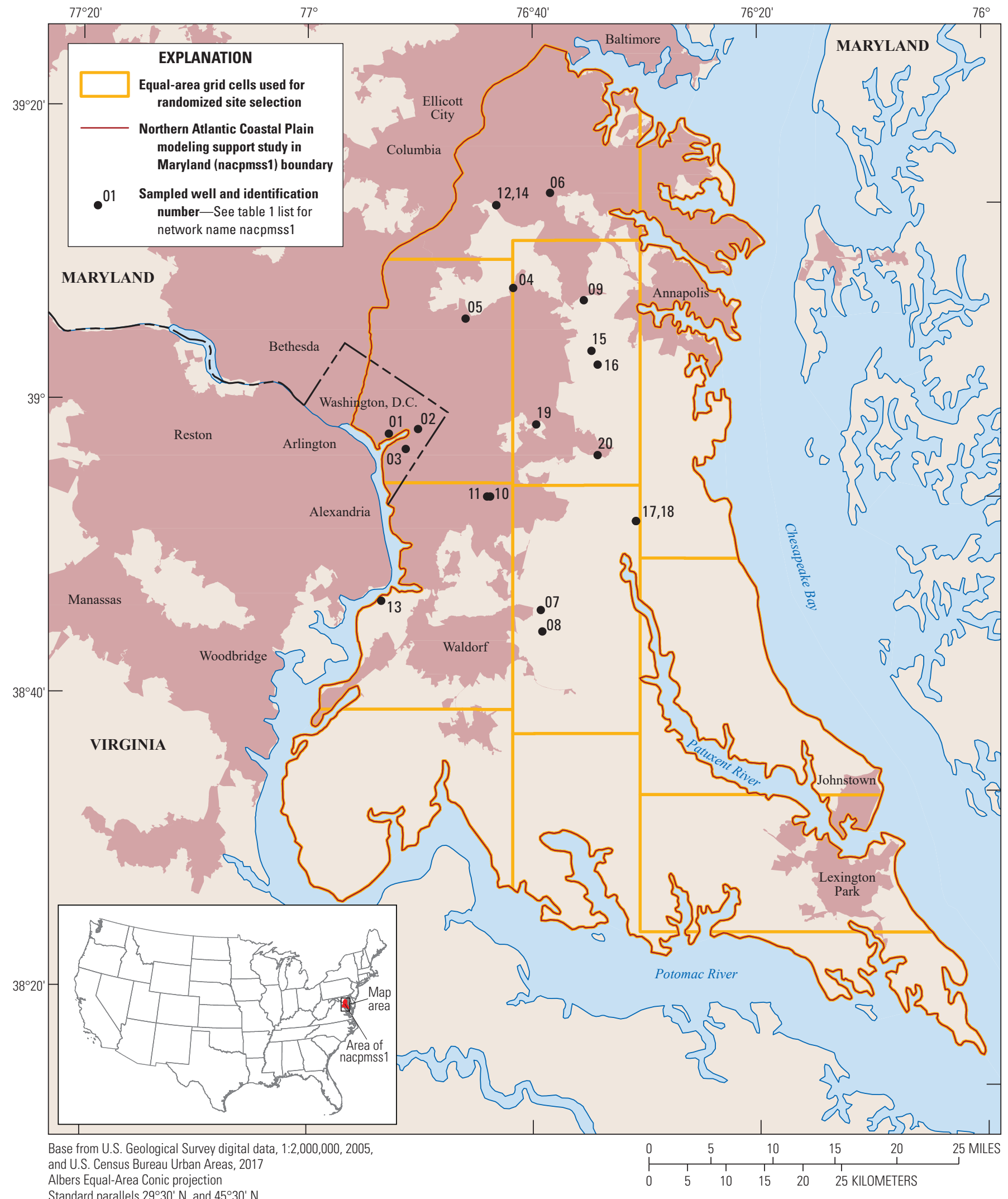

Albers Equal-Area Conic projection

Standard parallels $29^{\circ} 30^{\prime} \mathrm{N}$. and $45^{\circ} 30^{\prime} \mathrm{N}$.

Central meridian $96^{\circ} 00^{\prime} \mathrm{W}$.

North American Datum of 1983

Figure 24. Study and wells sampled as part of the Northern Atlantic Coastal Plain modeling support study network (nacpmss1) for the U.S. Geological Survey National Water-Quality Assessment Project. 


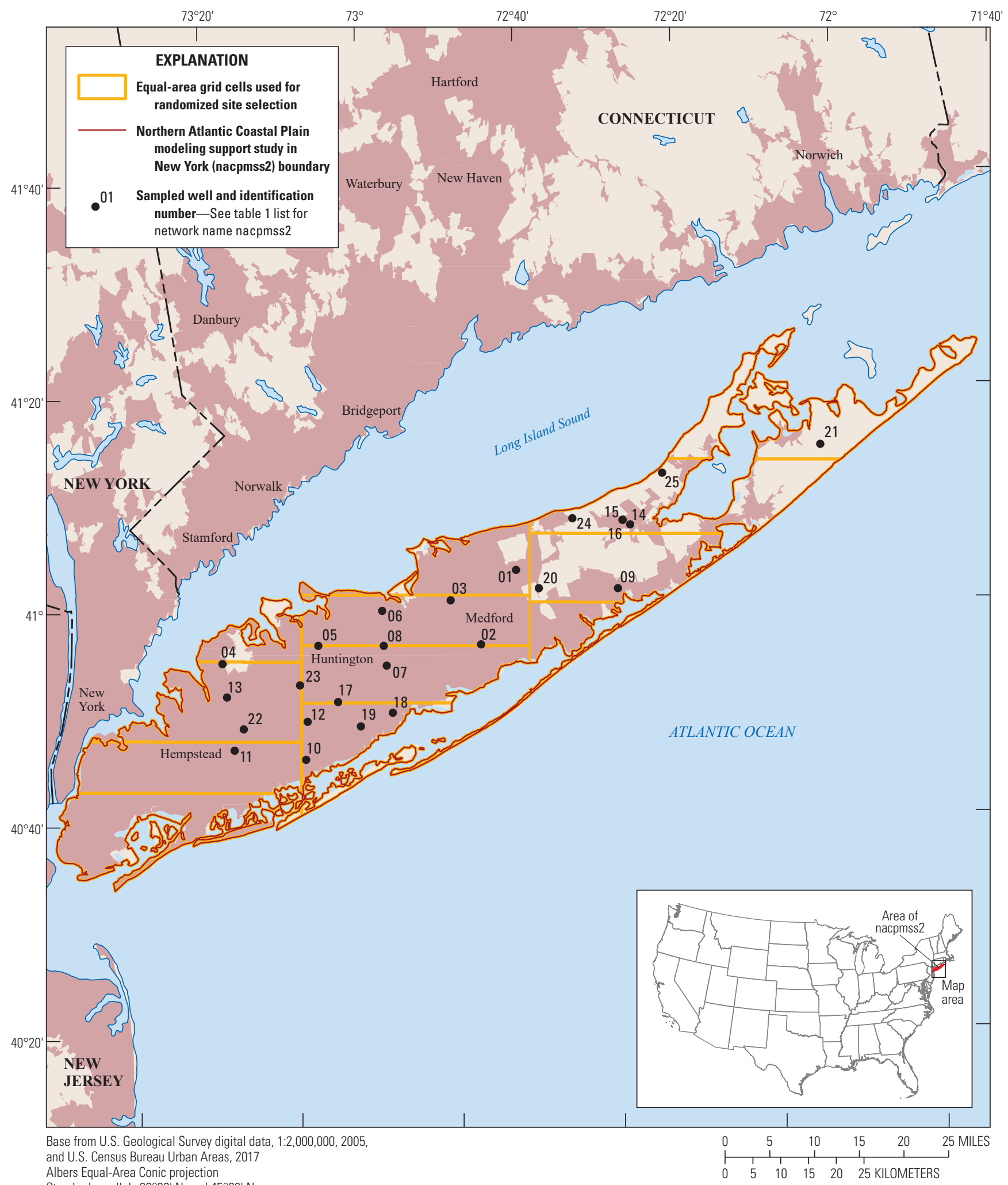

Standard parallels $29^{\circ} 30^{\prime} \mathrm{N}$. and $45^{\circ} 30^{\prime} \mathrm{N}$.

Central meridian $96^{\circ} 00^{\prime} \mathrm{W}$.

North American Datum of 1983

Figure 25. Study and wells sampled as part of the Northern Atlantic Coastal Plain modeling support study network (nacpmss2) for the U.S. Geological Survey National Water-Quality Assessment Project. 


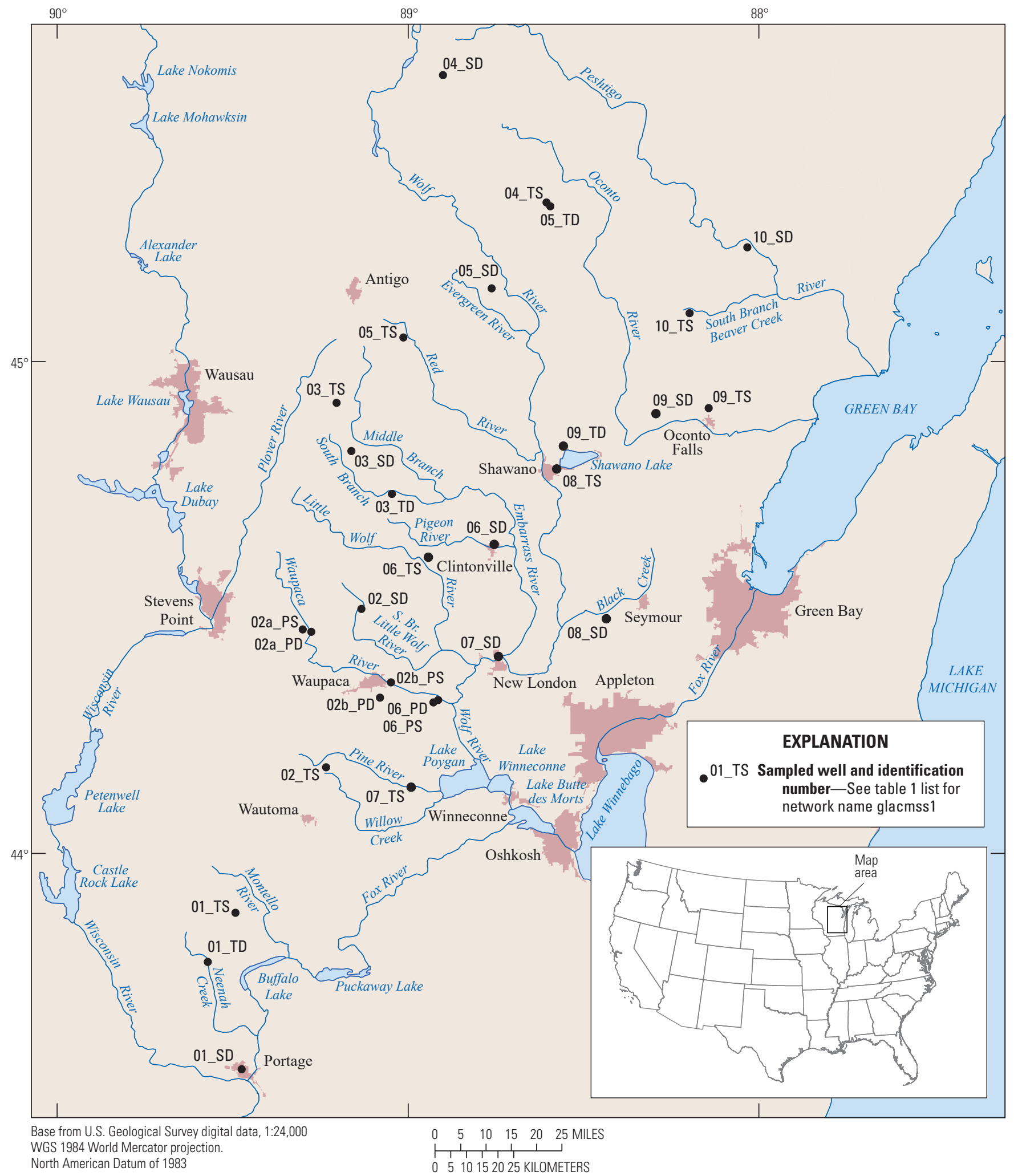

Figure 26. Study and wells sampled as part of the Glacial aquifer system modeling support study network (glacmss1) for the U.S. Geological Survey National Water-Quality Assessment Project. 


\section{Glacial Aquifer System Modeling Support Study Network (glacmss1)}

The Glacial aquifer system MSS network (glacmss1, fig. 24), in eastern Wisconsin, was designed to provide additional groundwater quality and age information for the parts of the glacial aquifer in the Fox-Wolf-Peshtigo groundwater model area that were not already covered by other NAWQA groundwater networks. More specifically, this network provides information about the deeper parts of the sand and gravel aquifer, and about both the shallow and deep parts of the till in the Fox-Wolf-Peshtigo model area. The network also provides some vertical flow path information for the sand and gravel aquifer.

The eastern part of the network area is underlain by east-sloping sedimentary rocks of Cambrian, Ordovician, and Silurian age (Batten and Bradbury, 1996). Rocks of Precambrian age, primarily granite, underlie the western part of the network area. Quaternary age unconsolidated deposits overlie the entire area, and these deposits are generally thickest along major river valleys (Batten and Bradbury, 1996; Soller and others, 2012). Groundwater is an important source of drinking water in the study area, especially for domestic wells. Both naturally occurring and anthropogenic contaminants adversely affect drinking-water quality in the area. In the Central Sands, nitrate is a common contaminant, while arsenic is common in parts of Paleozoic aquifers in eastern Wisconsin (Luczaj and Masarik, 2015).

The network includes a total of 30 wells: 10 deep sand and gravel wells, 6 shallow sand and gravel wells that are paired with deep sand and gravel wells, 10 shallow till wells, and 4 deep till wells. Well depths range from 45 to $245 \mathrm{ft}$ below ground surface. Samples for the model support study were collected August through September 2014, and these data are reported here.

\section{Northern Atlantic Coastal Plain Modeling Support Study Networks (nacpmss1, nacpmss2)}

There are two MSS networks in the Northern Atlantic Coastal Plain aquifer (nacpmss1, fig. 25; nacpmss2, fig. 26). One is in Maryland (nacpmss1), and the other one is in New York (nacpmss2). The nacpmss1 underlies and is to the east of the cities of Baltimore, Maryland, and Washington, D.C. This study is within the broader Northern Atlantic Coastal Plain principal aquifer setting described in Arnold and others, (Arnold and others, 2017b). It consists of unconsolidated to partly consolidated Early Cretaceous to Holocene age sediments that thicken and deepen toward the Atlantic Coast. Twenty wells ranging in depth from 60 to $645 \mathrm{ft}$ were sampled from regional layered aquifers in the Piney Point, Nanjemoy, Aquia, Magothy, Potomac, Patapsco (upper and lower), and Patuxent Formations (Shedlock and others, 2007).
The Northern Atlantic Coastal Plain aquifer modeling support study area in New York, nacpmss2, underlies the half of Long Island (split the long way) along the Atlantic Coast to the east and north of New York City. This study is within the broader Northern Atlantic Coastal Plain principal aquifer setting described in Arnold and others (2017b). Twenty-five wells ranging in depth from 200 to $900 \mathrm{ft}$ were sampled in the Magothy Formation regional layered aquifer.

\section{Sample Collection and Analysis}

Water-quality data from samples collected at 648 wells (fig. 1; table 1) are available in Arnold and others (2020). Groundwater samples were collected and processed using methods designed to yield samples that were representative of environmental conditions, minimally affected by contamination, and consistent nationwide (Koterba and others, 1995; Lapham and others, 1995; USGS, variously dated). All samples were collected at the wellhead (the point at which the groundwater exits the well near land surface) or as close to the wellhead as possible. This location was selected so that samples were collected before any treatment or blending potentially could alter constituent concentrations. Samples were collected and processed using prescribed protocols described in Koterba and others (1995), Lapham and others (1995), and the USGS National Field Manual (USGS, variously dated). Samples were analyzed at the USGS National Water-Quality Laboratory (NWQL) in Denver, Colorado, for water-quality indicators, nutrients and dissolved organic carbon, major and minor ions, trace elements, VOCs, pesticides, radon radiochemistry, and one item of special interest, arsenic speciation. Four radionuclide constituent concentrations (lead-210, polonium-210, radium-224, radium-226, and radium-228) were analyzed by ALS Environmental in Fort Collins, Colo. Perchlorate concentrations were analyzed by Weck Laboratories, Inc. in Industry, Calif. Hexavalent chromium (chromium [VI]) concentrations were analyzed by the USGS Trace Metal Laboratory in Boulder, Colo. Strontium concentrations were analyzed by the Metal and Metalloid Isotope Research Laboratory in Menlo Park, Calif.

The constituents for which samples were collected are listed in table 2 of Arnold and others (2020) and are organized by constituent class; constituent primary uses and sources; analytical schedules and sampling period; analytical method references; USGS parameter codes; comparison thresholds; reporting levels for the 2016 sampling period; number of analyses, detections, and detections above the reporting level; and the table in which the data for the constituent class are shown. The reporting levels shown are for the samples collected in 2016. Reporting levels for earlier data are shown in Arnold and others (2016b, 2017a, 2018a). Analytical schedules are groups of constituents for which laboratory analysis is requested. The USGS parameter code identifies the constituents, and the method reference indicates the laboratory method 
used to analyze the samples. The reported concentration of a constituent can be evaluated using the comparison threshold value. Of the comparison thresholds listed in table 2 of Arnold and others (2020), only the secondary maximum contaminant level is not health based.

In addition to discrete water-quality samples that are collected periodically, the ETN wells also are instrumented to measure basic water-quality parameters at a high frequency during specific periods throughout each day. Each well is instrumented with a water-quality sonde that contains temperature, specific conductance, $\mathrm{pH}$, and dissolved oxygen probes. Some wells also are instrumented to measure nitrate. The sonde sits in a flow-through chamber that receives groundwater flow from near the wellhead. Measurements of the basic water-quality parameters are made when the well is pumping and groundwater is flowing through the system, which may range from 1 to 24 hours per day. Waterquality data are recorded by the sonde at different intervals, from 2 minutes to 12 hours, depending on the network. The water-quality data are transmitted to a data collection platform where the data are stored and transmitted to the USGS National Water Information System (USGS, 2018) database by the Geostationary Operational Environmental Satellite network. The high-frequency data are reviewed, corrected, and approved according to recommendations for publishing continuous water-quality records (Wagner and others, 2006).

\section{Data Reporting}

Laboratories use specified values, referred to as reporting levels, in reporting results determined during analysis of water samples. Different reporting levels are used depending on the constituent and the laboratory method used to analyze the sample. Concentrations not measured above a certain threshold concentration for that constituent are reported as less than the reporting level; these are censored data.

Reporting levels are defined differently by the NWQL for inorganic and organic constituents. Inorganic constituents (major ions, nutrients, and trace elements) for samples analyzed in 2016 are each reported using a reporting level (RL) that is equivalent to the detection limit (DL). The DL is the smallest concentration that can be measured and reported with 99-percent confidence that the concentration is greater than zero, which means less than or equal to a 1-percent chance of a false positive (Williams and others, 2015). The DLs used in 2016 for both inorganic and organic constituents generally were determined using the DQCALC method described in Williams and others (2015); this is indicated as DLDQC in table 2 of Arnold and others (2020). However, for a few constituents that have commonly been detected in laboratory blank samples, the DLs were determined using an approach that calculates a DL directly from blank data (Williams and others, 2015); this is indicated as DLBLNK in table 2 of Arnold and others (2020). Organic constituents (VOCs and pesticides) are each reported using an RL that typically is about twice, but may be more than twice, the DL (Williams and others, 2015); this is indicated by RLDQC in table 2 of Arnold and others (2020) when calculated from a DL determined using the DQCALC method. This approach to setting the RL is estimated to limit the chance of incorrectly reporting the constituent as absent to less than or equal to 1 percent (Williams and others, 2015). In other words, there is at least a 99-percent confidence that the constituent really is absent from the sample when it is reported relative to the RL. The RLs are used for reporting analytical results for VOCs and pesticides to allow for the robust analysis and interpretation of detection frequencies. The NWQL uses information-rich analytical methods such as gas chromatography or high-performance liquid chromatography for these constituents and often provides results that indicate the presence of these constituents at concentrations less than their RLs, and even at concentrations less than their DLs.

A few constituents are reported using minimum reporting levels (MRLs). The MRLs are calculated according to the EPA definition of an MRL, described previously as the minimum concentration of a substance that can be measured and reported with 99-percent confidence that the value is above zero (Childress and others, 1999). An MRL is a reporting level that is chosen by the laboratory.

Radionuclides are reported using units of radioactive activity (picocuries per liter) rather than concentration. Reporting levels for these constituents are based on the sample-specific critical level (ssLc) or sample-specific minimum detectable concentrations (ssMDCs) (McCurdy and others, 2008). The ssLc and ssMDC are calculated for each sample from parameter values used during the actual analysis of the sample. The ssLc and ssMDC are analogous to the $\mathrm{DL}$ and RL, respectively. The ssLc is defined as the smallest measured activity that indicates detection of the radionuclide, with no more than a 5-percent chance of a false positive detection (EPA, 2004). The specified probability associated with a critical level can vary, but it is typically 5 percent for radionuclides. Like the DL, the ssLc is a reporting level that is based on a specified probability of false positive errors; that is, incorrectly reporting that the radionuclide is present. The ssMDC, like the RL, is a reporting level that is based on a specified probability of false negative errors; that is, failing to report that the radionuclide is present. The ssMDC is defined as the activity at which there is 5-percent chance of a false negative error and typically is about two times greater than the ssLc (McCurdy and others, 2008).

The laboratory method used to analyze samples for VOCs in schedules 4436 and 4437 was approved and published in 2016 (Rose and others, 2016). Results for laboratory methods that are unapproved generally are not made available to the public by the USGS because the quality of the results could be affected by problems subsequently discovered during the process of method approval; however, the method approval process revealed no substantial problems and resulted in no changes in the analytical process (Duane Wydoski, USGS, 
written commun., 2015). This indicates that the data reported before approval for these methods were of sufficient quality for public release.

Concentrations below DLs, and concentrations between DLs and RLs, are reported without any qualifiers in this report. Concentrations below DLs or between DLs and RLs can be identified by comparing the reported concentrations with the DLs and RLs listed by compound in table 2 of Arnold and others (2020). It is important to note that there is greater uncertainty associated with values less than DLs (regarding risk of false positive errors or inaccurate detections) and with values less than RLs (regarding risk of false negative values or inaccurate nondetections) than with values that are greater than DLs, RLs, or both.

The data presented in this report and associated data release (Arnold and others, 2020) are current as of the date of retrieval (December 18, 2018) from the National Water Information System (USGS, 2018). For example, RLs may be adjusted when additional QC data for the method are examined. The well information and water-quality data presented in this report and the associated data release were reviewed by USGS personnel and subsequently verified by coauthors who are responsible for tracking the data.

\section{Quality-Assurance and Quality-Control Methods}

The quality-assurance plan for NAWQA Project groundwater samples was derived from previous NAWQA Project cycles of study (Koterba and others, 1995) and the USGS National Field Manual (USGS, variously dated). About 14 percent of samples collected during any period are for data quality assurance and quality control (QC). Types of QC samples include equipment blanks, source solution blanks, field blanks, replicates, field spikes, and laboratory spikes. Data and results from statistical analysis of blank QC samples are presented in appendix 4 (tables 4.1 and 4.2).

Blanks are used to test for bias from an unintentional introduction of contamination to environmental samples. Equipment blanks are used to test whether equipment is clean and free of contamination. Source solution blanks are used to test whether the water used for the blank sample is free of contamination. Field blanks are used to test for contamination that may be introduced during sample collection, processing, handling, and analysis. Field blanks also are used to test for contamination from the environment around where the sample was collected. Replicates are samples that are collected at the same time and using the same method as the environmental sample. Replicates measure the variability of determining a concentration in samples that should be essentially identical. Spiked samples are used to measure the performance of analytical methods on an environmental water sample. A sample can be spiked in the field or the laboratory.
The number and type of QC samples planned for each network study depend on the number of wells sampled, the number of sampling teams that are involved in the sampling, and the constituents for which samples will be analyzed, as described in the following criteria:

- Equipment blanks are collected for nutrients, trace elements, and VOCs at the quantity of one blank for each team sampling the network.

- Source solution blanks are collected for nutrients, trace elements, and VOCs at the quantity of one blank for each team sampling the network. The VOCs have additional source solution blanks that are collected with each field blank.

- Field blanks are collected for major ions, nutrients, dissolved organic carbon, trace elements, and pesticides at the quantity of 1 blank for every 15 wells sampled or 1 blank for each team sampling the network (whichever results in a greater number of blanks). Field blanks are collected for VOCs at the quantity of 1 blank for every 10 wells sampled or 1 blank for each team sampling the network (whichever results in a greater number of blanks).

- Replicate samples are collected for major ions, nutrients, dissolved organic carbon, trace elements, VOCs, and radionuclides at the quantity of 1 replicate for every 30 wells sampled. Replicate samples are collected for pesticides at the quantity of 1 replicate for every 15 wells.

- Field spikes are collected for pesticides at the quantity of 1 spike sample for every 30 wells sampled.

- Laboratory spikes are collected for VOCs at the quantity of 1 spike sample for every 30 wells sampled.

Statistical analysis of QC sample data can be used to evaluate the variability or bias of the data, sampling and sample handling procedures, and laboratory and (or) field methods and to ensure the environmental assessment samples represent true groundwater chemistry. The QC sample data provided in Arnold and others (2020) include water quality for all blank QC samples collected between January and December 2016 in association with the environmental sample data and a few results from earlier sampling periods that were not previously published. Data from the 2012-13 sampling period are presented in Arnold and others (2016a,b), data from the 2014 sampling period are presented in Arnold and others (2017a,b), and data from the 2015 sampling period are presented in Arnold and others $(2018 \mathrm{a}, \mathrm{b})$. 


\section{Groundwater-Quality Data}

Groundwater-quality data from 648 wells are included in this report (table 1). Samples were analyzed for 376 constituents (table 2 of Arnold and others, 2020); however, not all wells were sampled for all constituents. Results of analyses are presented in tables 3-13 of Arnold and others (2020), which are organized by constituent class: water-quality indicators (table 3); nutrients and dissolved organic carbon (table 4); major and minor ions (table 5); trace elements (table 6); VOCs (tables 7); pesticides (table 8); radiochemistry (table 9); and special-interest constituents, including arsenic speciation (table 10), chromium (VI) (table 11), perchlorate (table 12), and strontium (table 13). The constituents for which samples were analyzed and the table in which the data are presented are listed in table 2 of Arnold and others (2020). Comparative benchmarks (thresholds) listed in that table provide context for evaluating the constituent concentration data in terms of human health and other characteristics relevant for drinkingwater use. Several types of thresholds are listed. The EPA maximum contaminant levels are legally enforceable drinkingwater standards that specify the maximum permissible level of a constituent that can be delivered to a user of a public water system. The EPA human-health benchmarks for pesticides (HHBPs) are nonenforceable screening levels for evaluating if a pesticide concentration in drinking-water sources may indicate a potential human-health risk (EPA, 2012). The HHBPs include benchmarks for cancer and noncancer health effects (EPA, 2013). The USGS Health-Based Screening Levels are nonenforceable benchmarks for constituents that do not have HHBPs or maximum contaminant levels (MCLs) that can be used to evaluate if constituent concentrations may indicate a potential human-health concern (Toccalino, 2007; Toccalino and others, 2014). Like EPA HHBPs, USGS health-based screening levels are categorized in terms of cancer and noncancer health effects.

The groundwater-quality data from January to December 2016 are presented in the format of tab-delimited ASCII text files and are available for download from Arnold and others (2020) along with complete metadata files that describe the contents of each text file. The data may be imported into a spreadsheet, database, or statistical software for manipulation and analysis. The data available from Arnold and others (2020) are referenced as tables 1-13 and appendix tables $4.10-4.19$ in this report.

\section{Water-Quality Indicators}

Water-quality indicators include water temperature, dissolved oxygen, specific conductance, $\mathrm{pH}$, alkalinity, carbonate and bicarbonate (calculated from alkalinity), and turbidity (table 3 of Arnold and others, 2020). Water-quality indicators are measured in the field when the other water samples are collected (USGS, variously dated), and $\mathrm{pH}$ and specific conductance sometimes are also measured in the laboratory.
Water-quality indicators provide basic information about the general quality and geochemical conditions of the water. Dissolved oxygen is the concentration of oxygen dissolved in the water and is an indicator of reduction-oxidation (redox) conditions in the aquifer. Measurements of $\mathrm{pH}$ indicate the acidity or basicity of water. Dissolved oxygen and $\mathrm{pH}$ are important controls on the chemical reactions that can happen in water. Specific conductance is a measure of how well the water conducts electricity and indicates the relative amount of dissolved solids in the water. Alkalinity, carbonate, and bicarbonate indicate the hardness of water and are related to $\mathrm{pH}$. Turbidity is a measure of the suspended solids in the water.

\section{Inorganic Constituents}

Inorganic constituents are most often naturally present in groundwater. Groundwater samples were analyzed for the following inorganic constituent classes: major and minor ions, nutrients and dissolved organic carbon, and trace elements (including metals; tables 4-6 of Arnold and others, 2020).

Nutrients include nitrogen and phosphorus compounds and dissolved organic carbon. Data for ammonia, nitrate plus nitrite, nitrite, total nitrogen, and phosphorus measured as orthophosphate are presented in table 4 of Arnold and others (2020). Nutrients are present naturally, but nutrient concentrations also are affected by human activities such as farming and wastewater disposal (Hem, 1992). Nitrogen was measured as total nitrogen and as the individual nitrogen species of nitrite, nitrate, and ammonia. Nutrient concentrations can affect the quality of groundwater for use as drinking water.

Major and minor ions are cations and anions that can be dissolved in water from geologic materials. Concentrations of major and minor ions can be used to classify water into different types (Hem, 1992; Hiscock, 2005). Waters with similar ion concentrations often have similar history, recharge areas, climate, mineralogy, and residence time (Güler and others, 2002). Some major ions can affect the quality of water for drinking and other uses. Groundwater samples were analyzed for 10 major and minor ions and total dissolved solids (table 5 of Arnold and others, 2020).

Trace elements consist of metals that are usually present in the environment in very small quantities (Hem, 1992). Trace elements often are dissolved in water from geologic materials, but concentrations of these elements also can be affected by human activities such as mining. Many trace elements can affect the quality of groundwater for use as drinking water. Groundwater samples were analyzed for 22 trace elements (table 6 of Arnold and others, 2020).

\section{Organic Compounds}

Organic compounds are man-made chemicals and include VOCs and pesticides. VOCs are chemicals that tend to evaporate into the air and are in a variety of substances including disinfectants, solvents, paint, fumigants, asphalt, and fuel 
additives (Zagorsky and others, 2006). Pesticides are chemical compounds used to control plant or insect pests and include fungicides, herbicides, and insecticides (Gilliom and others, 2006). Many VOCs and pesticides, if present, can affect the quality of groundwater used for drinking water. Groundwater samples from 2016 were analyzed for 85 VOCs and 225 pesticides (tables 7 and 8 of Arnold and others, 2020).

\section{Radiochemistry}

Radiochemical constituents include radionuclides and measurements of radioactivity. Radionuclides are chemical constituents that are produced naturally by the decay of radioactive parent elements such as uranium and thorium. Sources of radionuclides in groundwater are geologic material such as rocks and soils (Hem, 1992). Radionuclides and measurements of radioactivity included in this report are $\alpha$ radioactivity, $\beta$ radioactivity, radon (a dissolved gas), several isotopes of radium (radium-224, radium-226, and radium-228), polonium-210, and lead-210 (table 9 of Arnold and others, 2020). Uranium, which also is a radionuclide, is included with trace elements (table 6 of Arnold and others, 2020) because uranium is measured in units of mass concentration rather than as units of radioactivity. In total, groundwater samples were analyzed for eight radionuclides and measures of radioactivity.

\section{Constituents of Special Interest}

Several constituents of special interest were included for selected networks. Constituents of special interest were arsenic species (arsenate, arsenite, monomethylarsonate, and dimethylarsinate), which are derived from arsenic, hexavalent chromium (chromium [VI]), perchlorate, and strontium isotopes ( ${ }^{87} \mathrm{Sr} /{ }^{86} \mathrm{Sr}$ ) (tables $10-13$ of Arnold and others, 2020). Arsenic and chromium are predominantly natural in origin but may have localized anthropogenic sources. Anthropogenic arsenic uses include metal and ore processing, glass production, fossil fuel combustion, wood preservatives, pesticides, semiconductor production, and pharmaceuticals (Garelick and others, 2008). Hexavalent chromium is chromium in the +6 oxidation state (six electrons lost from the atom) and is used in textile dyes, wood preservation, anticorrosive agents, and other surface coatings (Nriagu and Niebor, 1988). Geochemical conditions such as redox and $\mathrm{pH}$ affect the speciation of chromium and arsenic in groundwater (Hem, 1992). Most arsenic and chromium in groundwater is from geologic sources in rocks and soils. Perchlorate is an inorganic constituent used in rocket fuels, fireworks, safety flares, and other products; it is present in some fertilizers and may be present naturally at low concentrations in groundwater (Srinivasan and Sorial, 2009; Jackson and others, 2015). Strontium isotope variations in groundwater provide insight into the sources of dissolved constituents to groundwater and have been used to trace flow paths and mineral-solution reactions in soils and aquifer rocks (Banner, 2004).

\section{Summary}

As part of the third decadal cycle of the U.S. Geological Survey National Water-Quality Assessment Project, groundwater-quality data are being collected from well networks to assess water-quality conditions in the Nation's principal aquifers and investigate changes in groundwater-quality conditions in selected land use and hydrogeologic settings. Groundwater-quality data are published in annual data series reports, of which this report is the third in the series.

Groundwater-quality data from 648 wells were collected from 7 types of well networks: principal aquifer study networks, land-use study networks, major aquifer study networks, enhanced trends networks, vertical flow-path study networks, flow-path study (FPS), and modeling support study (MSS). Within principal aquifer, land-use, and major aquifer study networks, study areas were divided into equal-area grid cells and wells were selected for sampling using a stratified random sampling design. The number of wells in principal aquifer networks ranged from about 40 to 60 wells per network for the studies included in this report. About 30 wells typically made up each land-use or major aquifer study network. Enhanced trends networks that were sampled in 2016 consisted of two to five wells that were selected at locations within aquifers where temporal changes in groundwater quality might be expected. Three vertical flow-path study network, four flow-path studies, and three modeling support studies are described in this report.

Groundwater samples were analyzed for water-quality indicators and constituents, including nutrients, major and minor ions, trace elements, volatile organic compounds, pesticides, radiochemistry, and select special-interest constituents such as arsenic speciation, hexavalent chromium, and perchlorate. These groundwater-quality data are tabulated in this report and associated data release. Quality-control samples were collected along with environmental samples, and data from blank quality-control samples also are included in this report. The data release includes data collected during 2015 and previously unpublished data from selected environmental samples collected in 2013 and selected quality-control samples collected in 2014. These previously unpublished data are associated with networks described in this report. 


\section{Acknowledgments}

Many people in addition to the authors participated in providing information for this report. Bryant Jurgens and Tyler Johnson (both with the U.S. Geological Survey) provided tools for data compilation. Personnel in U.S. Geological Survey water science centers, too numerous to name individually, collected, packaged, and shipped water samples to the laboratories for analysis and ensured results were entered into the U.S. Geological Survey National Water Information System.

\section{References Cited}

Anderholm, S.K., 1997, Water-quality assessment of the Rio Grande Valley, Colorado, New Mexico, and Texas-Shallow ground-water quality and land use in the Albuquerque area, central New Mexico, 1993: U.S. Geological Survey Water-Resources Investigations Report 97-4067, 73 p.

Anderholm, S.K., 2002, Water-quality assessment of the Rio Grande Valley, Colorado, New Mexico, and TexasSurface-water quality, shallow ground-water quality, and factors affecting water quality in the Rincon Valley, southcentral New Mexico, 1994-95: U.S. Geological Survey Water-Resources Investigations Report 02-4188, 117 p.

Andrews, W.J., Fong, A.L., Harrod, L., and Dittes, M.E., 1997, Water-quality assessment of part of the upper Mississippi River basin, Minnesota and Wisconsin-Ground-water quality in an urban part of the Twin Cities Metropolitan area, Minnesota, 1996: U.S. Geological Survey WaterResources Investigations Report 97-4248, 54 p., accessed February 2020 at https://doi.org/10.3133/wri974248.

Arnold, T.L., Bexfield, L.M., Musgrove, M., Lindsey, B.D., Stackelberg, P.E., Barlow, J.R., DeSimone, L.A., Kulongoski, J.T., Kingsbury, J.A., Ayotte, J.D., Fleming, B.J., and Belitz, K., 2017a, Datasets from groundwater quality data from the National Water Quality Assessment Project, January through December 2014 and select qualitycontrol data from May 2012 through December 2014: U.S. Geological Survey data release, accessed March 28, 2018, at https://doi.org/10.5066/F7W0942N.

Arnold, T.L., Bexfield, L.M., Musgrove, M., Lindsey, B.D., Stackelberg, P.E., Barlow, J.R., DeSimone, L.A., Kulongoski, J.T., Kingsbury, J.A., Ayotte, J.D., Fleming, B.J., and Belitz, K., 2017b, Groundwater-quality data from the National Water-Quality Assessment Project, January through December 2014 and select quality-control data from May 2012 through December 2014: U.S. Geological Survey Data Series 1063, 83 p., accessed March 28, 2018, at https://doi.org/10.3133/ds1063.
Arnold, T.L., Bexfield, L.M., Musgrove, M., Stackelberg, P.E., Lindsey, B.D., Kingsbury, J.A., Kulongoski, J.T., and Belitz, K., 2018b, Groundwater-quality and select qualitycontrol data from the National Water-Quality Assessment Project, January through December 2015, and previously unpublished data from 2013 to 2014: U.S. Geological Survey Data Series 1087, 68 p., accessed February 2020 at https://doi.org/10.3133/ds1087.

Arnold, T.L., Bexfield, L.M., Musgrove, M., Stackelberg, P.E., Lindsey, B.D., Kingsbury, J.A., Kulongoski, J.T., Belitz, K., and Sharpe, J.B., 2018a, Datasets from groundwaterquality and select quality-control data from the National Water-Quality Assessment Project, January through December 2015 and previously unpublished data from 2013-2014: U.S. Geological Survey data release, accessed September 4, 2019, at https://doi.org/10.5066/F7XK8DHK.

Arnold, T.L., Bexfield, L.M., Musgrove, M., Stackelberg, P.E., Lindsey, B.D., Kingsbury, J.A., Kulongoski, J.T., Belitz, K., and Sharpe, J.B., 2020, Datasets from groundwaterquality and select quality-control data from the National Water-Quality Assessment Project, January through December 2016, and previously unpublished data from 2013 to 2015: U.S. Geological Survey data release, accessed February 2020 at https://doi.org/10.5066/P9W4RR74.

Arnold, T.L., DeSimone, L.A., Bexfield, L.M., Lindsey, B.D., Barlow, J.R., Kulongoski, J.T., Musgrove, M., Kingsbury, J.A., and Belitz, K., 2016b, Groundwater quality data from the National Water-Quality Assessment Project, May 2012 through December 2013: U.S. Geological Survey Data Series 997, 56 p., accessed March 28, 2018, at https://doi.org/10.3133/ds997.

Arnold, T.L., DeSimone, L.A., Bexfield, L.M., Lindsey, B.D., Barlow, J.R., Kulongoski, J.T., Musgrove, M., Kingsbury, J.A., and Belitz, K., 2016a, Groundwater quality data from the National Water Quality Assessment Project, May 2012 through December 2014 and select quality-control data from May 2012 through December 2013: U.S. Geological Survey data release, accessed March 28, 2018, at https://doi.org/ 10.5066/F7HQ3X18.

Banner, J.L., 2004, Radiogenic isotopes-Systematics and applications to earth surface processes and chemical stratigraphy: Earth-Science Reviews, v. 65, nos. 3-4, p. 141-194. [Also available at https://doi.org/10.1016/ S0012-8252(03)00086-2.]

Batten, W.G., and Bradbury, K.R., 1996, Regional groundwater flow system between the Wolf and the Fox Rivers near Green Bay, Wisconsin: Wisconsin Geological and Natural History Survey Information Circular 75, 28 p. 
Belitz, K., Jurgens, B., Landon, M.K., Fram, M.S., and Johnson, T., 2010, Estimation of aquifer scale proportion using equal area grids-Assessment of regional scale groundwater quality: Water Resources Research, v. 46, no. 11, 14 p., accessed March 15, 2017, at https://doi.org/ 10.1029/2010WR009321.

Burow, K.R., and Belitz, K., 2014, Groundwater studiesPrincipal aquifer surveys: U.S. Geological Survey Fact Sheet 2014-3024, 2 p., accessed January 4, 2016, at https://doi.org/10.3133/fs20143024.

Burow, K.R., Shelton, J.L., and Dubrovsky, N.M., 2008, Regional nitrate and pesticide trends in ground water in the eastern San Joaquin Valley, California: Journal of Environmental Quality, v. 37, S5, p. S-249-S-263. [Also available at https://doi.org/10.2134/jeq2007.0061.]

Burow, K.R., Panshin, S.Y., Dubrovsky, N.H., Vanbrocklin, D., and Fogg, G.E., 1999, Evaluation of processes affecting 1,2-dibromo-3-chloropropane (DBCP) concentrations in ground water in the eastern San Joaquin Valley, California-Analysis of chemical data and ground-water flow and transport simulations: U.S. Geological Survey Water-Resources Investigations Report 99-4059, 57 p.

Campbell, B.G., and Landmeyer, J.E., 2014, Groundwater availability in the Crouch Branch and McQueen Branch aquifers, Chesterfield County, South Carolina, 1900-2012: U.S. Geological Survey Scientific Investigations Report 2014-5050, 80 p., accessed February 2020 at https://doi.org/ $10.3133 /$ sir20145050.

Childress, C.J.O., Forman, W.T., Connor, B.F., and Maloney, T.J., 1999, New reporting procedures based on long-term method detection levels and some considerations for interpretations of water-quality data provided by the U.S. Geological Survey National Water Quality Laboratory: U.S. Geological Survey Open-File Report 99-193, 19 p., accessed January 4, 2016, at https://water.usgs.gov/owq/ OFR_99-193/index.html.

Fleming, A.H., 1994, The hydrogeology of Allen County, Indiana-A geologic and ground-water atlas: Indiana Geological Survey Special Report 57, 111 p.

Frans, L.M., Rupert, M.G., Hunt, C.D., Jr., and Skinner, K.D., 2012, Groundwater quality in the Columbia Plateau, Snake River Plain, and Oahu basaltic-rock and basin-fill aquifers in the northwestern United States and Hawaii, 1992-2010: U.S. Geological Survey Scientific Investigations Report 2012-5123, 84 p. [Also available at https://doi.org/10.3133/ sir20125123.]
Garelick, H., Jones, H., Agnieszka, D., and Valsami-Jones, E., 2008, Arsenic pollution sources, in Garelick, H., and Jones, H., eds., Reviews of environmental contamination and toxicology-Arsenic pollution and remediation-An international perspective: Springer-Verlag New York, Reviews of Environmental Contamination and Toxicology, v. 197, p. 17-60.

Gilliom, R.J., Alley, W.M., and Gurtz, M.E., 1995, Design of the National Water-Quality Assessment ProgramOccurrence and distribution of water-quality conditions: U.S. Geological Survey Circular 1112, 33 p. accessed March 1, 2016, at https://doi.org/10.3133/cir1112.

Gilliom, R.J., Barbash, J.E., Crawford, C.G., Hamilton, P.A., Martin, J.D., Nakagaki, N., Nowell, L.H., Scott, J.C., Stackelberg, P.E., Thelin, G.P., and Wolock, D.M., 2006, The quality of our Nation's waters-Pesticides in the Nation's streams and ground water, 1992-2001: U.S. Geological Survey Circular 1291, 172 p., accessed August 9, 2017, at https://pubs.usgs.gov/circ/2005/1291/.

Güler, C., Thyne, G.D., McCray, J.E., and Turner, A.K., 2002, Evaluation of graphical and multivariate statistical methods for classification of water chemistry data: Hydrogeology Journal, v. 10, no. 4, p. 455-474. [Also available at https://doi.org/10.1007/s10040-002-0196-6.]

Hawley, J.W., and Haase, C.S., 1992, Hydrogeologic framework of the northern Albuquerque Basin: Socorro, New Mexico Bureau of Mines and Mineral Resources Open-File Report 387, variously paged.

Hays, P.D., Knierim, K.J., Breaker, B., Westerman, D.A., and Clark, B.R., 2016, Hydrogeology and hydrologic conditions of the Ozark Plateaus aquifer system: U.S. Geological Survey Scientific Investigations Report 2016-5137, 61 p., accessed February 2020 at https://doi.org/10.3133/ sir20165137.

Hem, J.D., 1992, Study and interpretation of the chemical characteristics of natural water: U.S. Geological Survey Water-Supply Paper 2254, 264 p.

Hiscock, K.M., 2005, Hydrogeology-Principles and practice: United Kingdom, Blackwell Publishing, 389 p.

Homer, C.G., Dewitz, J.A., Yang, L., Jin, S., Danielson, P., Xian, G., Coulston, J., Herold, N.D., Wickham, J.D., and Megown, K., 2015, Completion of the 2011 National Land Cover Database for the conterminous United StatesRepresenting a decade of land cover change information: Photogrammetric Engineering and Remote Sensing, v. 81, no. 5, p. 345-354. 
Hughes, W.B., Abrahamsen, T.A., Maluk, T.L., Reuber, E.J., and Wilhelm, L.J., 2000, Water quality in the Santee River basin and coastal drainages, North and South Carolina, 1995-98: Geological Survey Circular 1206, 32 p., accessed February 2020 at https://doi.org/10.3133/cir1206.

Inkpen, E.L., Tesoriero, A.J., Ebbert, J.C., Silva, S.R., and Sandstrom, M.W., 2000, Ground-Water Quality in Regional, Agricultural, and Urban Settings in the Puget Sound Basin, Washington and British Columbia, 1996-1998: U.S. Geological Survey Water-Resources Investigations Report 00-4100.

Jackson, W.A., Bohlke, J.K., Andraski, B.J., Fahlquist, L., Bexfield, L.M., Eckardt, F.D., Gates, J.B., Davila, A.F., McKay, C.P., Rao, B., Sevanthi, R., Rajagopalan, S., Estrada, N., Sturchio, N., Hatzinger, P.B., Anderson, T.A., Orris, G., Betahcourt, J., Stonestrom, D., Latorre, C., Li, Y., and Harvey, G.J., 2015, Global patterns and environmental controls of perchlorate and nitrate co-occurrence in arid and semi-arid environments: Geochimica et Cosmochimica Acta, v. 164, p. 502-522. [Also available at https://doi.org/ 10.1016/j.gca.2015.05.016.]

Jones, M.A., 1999, Geologic framework for the Puget Sound aquifer system, Washington and British Columbia: U.S. Geological Survey Professional Paper 1424-C, 31 p.

Kahle, S.C., Morgan, D.S., and Welch, W.B., 2011, Hydrogeologic framework and hydrologic budget components of the Columbia Plateau Regional Aquifer System, Washington, Oregon, and Idaho: U.S. Geological Survey Scientific Investigations Report 2011-5124, 66 p., accessed February 2020 at https://pubs.usgs.gov/sir/2011/5124/.

Kingsbury, J.A., Barlow, J.R.B., Jurgens, B.C., McMahon, P.B., and Carmichael, J.K., 2017, Fraction of young water as an indicator of aquifer vulnerability along two regional flow paths in the Mississippi Embayment aquifer system, southeastern USA: Hydrogeology Journal, v. 25, no. 6, p. 1661-1678. [Also available at https://doi.org/10.1007/ s10040-017-1566-4.]

Koterba, M.T., Wilde, F.D., and Lapham, W.W., 1995, Groundwater data-collection protocols and procedures for the National Water-Quality Assessment ProgramCollection and documentation of water-quality samples and related data: U.S. Geological Survey Open-File Report 95-399, 113 p., accessed January 4, 2016, at https://pubs.usgs.gov/of/1995/ofr-95-399/.

Lapham, W.W., Wilde, F.D., and Koterba, M.T., 1995, Ground-water data-collection protocols and procedures for the National Water-Quality Assessment ProgramSelection, installation, and documentation of wells, and collection of related data: U.S. Geological Survey OpenFile Report 95-398, 71 p., accessed January 4, 2016, at https://pubs.usgs.gov/of/1995/ofr-95-398/.
Luczaj, J., and Masarik, K., 2015, Groundwater quantity and quality issues in a water-rich region-Examples from Wisconsin, USA: Resources, v. 4, no. 2, p. 323-357. [Also available at https://doi.org/10.3390/resources4020323.]

Maupin, M.A., and Barber, N.L., 2005, Estimated withdrawals from principal aquifers in the United States, 2000: U.S. Geological Survey Circular 1279, 46 p., accessed January 4, 2016, at https://pubs.usgs.gov/circ/2005/1279/.

McCurdy, D.E., Garbarino, J.R., and Mullin, A.H., 2008, Interpreting and reporting radiological water-quality data: U.S. Geological Survey Techniques and Methods, book 5, chap. B6, 33 p., accessed January 4, 2016, at https://pubs.usgs.gov/tm/05b06/.

Meyer, G.N., 1993, Geologic sensitivity of the uppermost aquifer to pollution, in Meyer, G.N., Falteisek, J., Meints, J., and Dahlman, B., eds., Regional hydrogeologic assessment, Anoka Sand Plain, Anoka, Chisago, Isanti, and Sherburne Counties, Minnesota: Minnesota Department of Natural Resources Regional Hydrogeologic Assessment, RHA-1, 3 pls.

Miller, J.A., 1990, Ground water atlas of the United StatesAlabama, Florida, Georgia, South Carolina: U.S. Geological Survey Hydrologic Atlas 730-G, accessed January 4, 2016, at https://pubs.usgs.gov/ha/ha730/ch_g/index.html.

Miller, J.A., and Appel, C.L., 1997, Ground water atlas of the United States-Segment 3, Kansas, Missouri, Nebraska: U.S. Geological Survey Hydrologic Atlas 730-D, accessed December 8, 2017, at https://doi.org/10.3133/ha730D.

Nriagu, J.O., and Niebor, E., eds., 1988, Chromium in the natural and human environments: New York, John Wiley \& Sons, $571 \mathrm{p}$.

Peigat, J., 1989, Sensitivity of ground-water systems to pollution, in Balaban, N.H., ed., Atlas Hennepin County, Minnesota: Minnesota Geological Survey, County Atlas Series C-4, 8 pls., accessed February 2, 2020 at https://conservancy.umn.edu/bitstream/handle/11299/58491/ plate7_poll_sens.pdf? sequence $=95 \&$ isAllowed $=\mathrm{y}$.

Plummer, L.N., Bexfield, L.M., Anderholm, S.K., Sanford, W.E., and Busenberg, E., 2004, Geochemical characterization of ground-water flow in the Santa Fe Group aquifer system, Middle Rio Grande Basin, New Mexico: U.S. Geological Survey Water-Resources Investigations Report 03-4131, $395 \mathrm{p}$.

Renken, R.A., 1998, Ground water atlas of the United States-Segment 5, Arkansas, Louisiana, Mississippi: U.S. Geological Survey Hydrologic Atlas 730-F., accessed December 8, 2017, at https://doi.org/10.3133/ha730F. 
Rose, D.L., Sandstrom, M.W., and Murtagh, L.K., 2016, Determination of heat purgeable and ambient purgeable volatile organic compounds in water by gas chromatography/mass spectrometry: U.S. Geological Survey Techniques and Methods, book 5, chap. B12, 62 p. [Also available at https://doi.org/10.3133/tm5B12.]

Rowe, G.L., Jr., Belitz, K., Demas, C.R., Essaid, H.I., Gilliom, R.J., Hamilton, P.A., Hoos, A.B., Lee, C.J., Munn, M.D., and Wolock, D.W., 2013, Design of cycle 3 of the National Water-Quality Assessment Program, 2013-23-Part 2 - Science plan for improved water-quality information and management: U.S. Geological Survey Open File Report 2013-1160, 110 p., accessed January 4, 2016, at https:/pubs.usgs.gov/of/2013/1160/.

Rowe, G.L., Jr., Belitz, K., Essaid, H.I., Gilliom, R.J., Hamilton, P.A., Hoos, A.B., Lynch, D.D., Munn, M.D., and Wolock, D.W., 2010, Design of cycle 3 of the National Water-Quality Assessment Program, 2013-2023Part 1-Framework of water-quality issues and potential approaches: U.S. Geological Survey Open-File Report 2009-1296, 54 p., accessed January 4, 2016, at https:/pubs.usgs.gov/of/2009/1296/.

Rupert, M.G., 1997, Nitrate (NO2+NO3-N) in Ground Water of the Upper Snake River Basin, Idaho and Western Wyoming, 1991-95: U.S. Geological Survey WaterResources Investigations Report 97-4174, 56 p.

Saad, D.A., 2008, Agriculture-related trends in groundwater quality of the glacial deposits aquifer, central Wisconsin: Journal of Environmental Quality, v. 37, no. S5, p. S-209-S-225, accessed February 2020 at https://doi.org/ 10.2134/jeq2007.0053.

Scott, J.C., 1990, Computerized stratified random siteselection approaches for design of a ground-water-quality sampling network: U.S. Geological Survey Water-Resources Investigations Report 90-4101, 109 p., accessed January 4, 2016, at https://doi.org/10.3133/wri904101.

Shedlock, R.J., Bolton, D.W., Cleaves, E.T., Gerhart, J.M., and Nardi, M.R., 2007, A Science Plan for a Comprehensive Regional Assessment of the Atlantic Coastal Plain Aquifer System in Maryland: U.S. Geological Survey Open-File Report 2007-1205, 29 p., accessed February 2020 at https://doi.org/10.3133/ofr20071205.

Skinner, K.D., 2003, Probability of Detecting Elevated Concentrations of Nitrate in Ground Water in a Six-County Area of South-Central Idaho: U.S. Geological Survey Water-Resources Investigations Report 03-4143, 29 p.
Soller, D.R., Packard, P.H., and Garrity, C.P., 2012, Database for USGS Map I-1970-Map showing the thickness and character of Quaternary sediments in the glaciated United States east of the Rocky Mountains: U.S. Geological Survey Data Series 38, 656 p. [Also available at https://doi.org/ $10.3133 /$ ds656.]

Srinivasan, R., and Sorial, G.A., 2009, Treatment of perchlorate in drinking water-A critical review: Separation and Purification Technology, v. 69, no. 1, p. 7-21. [Also available at https://doi.org/10.1016/j.seppur.2009.06.025.]

Staubitz, W.W., Bortleson, G.C., Semans, S.D., Tesoriero, A.J., and Black, R.W., 1997, Water-quality assessment of the Puget Sound Basin, Washington-Environmental setting and its implications for water quality and aquatic biota: U.S. Geological Survey Water-Resources Investigations Report 97-4013, 76 p.

Tesoriero, A.J., Duff, J.H., Saad, D.A., Spahr, N.E., and Wolock, D.M., 2013, Vulnerability of streams to legacy nitrate sources: Environmental Science \& Technology, v. 47, no. 8, p. 3623-3629. [Also available at https://doi.org/ 10.1021/es305026x.]

Tesoriero, A.J., and Voss, F.D., 1997, Predicting the probability of elevated nitrate concentrations in the Puget Sound Basin Implications for aquifer susceptibility and vulnerability: Ground Water, v. 35, no. 6, p. 1029-1038.

Thomas, M.A., 2000a, Ground-water quality and vulnerability to contamination in selected agricultural areas of southeastern Michigan, northwestern Ohio, and northeastern Indiana: U.S. Geological Survey Water-Resources Investigations Report 00-4146, 22 p.

Thomas, M.A., 2000b, The effect of residential development on ground-water quality near Detroit, Michigan: Journal of the American Water Resources Association, v. 36, no. 5, p. 1023-1038. [Also available at https://doi.org/10.1111/ j.1752-1688.2000.tb05707.x.]

Toccalino, P.L., 2007, Development and application of healthbased screening levels for use in water-quality assessments: U.S. Geological Survey Scientific Investigations Report 2007-5106, 12 p., accessed January 4, 2016, at https://pubs.usgs.gov/sir/2007/5106/.

Toccalino, P.L., Norman, J.E., and Schoephoester, K.M., 2014, Health-based screening levels for evaluating waterquality data: U.S. Geological Survey data release, accessed January 4, 2016, at https://doi.org/10.5066/F71C1TWP.

U.S. Census Bureau, 2010, Census of population and housing, 2010: U.S. Census Bureau, digital data, accessed March 1, 2018, at https://www.census.gov/prod/www/decennial.html. 
U.S. Environmental Protection Agency [EPA], 2004, Multi-agency radiological laboratory analytical protocols manual (MARLAP) - Part II-Chapters 18-20, appendix G (volume III): U.S. Environmental Protection Agency, EPA 402-B-04-001C, accessed May 19, 2015, at https://nepis.epa.gov/Exe/ZyPDF.cgi/P10094KI.PDF? Dockey=P10094KI.PDF.

U.S. Environmental Protection Agency [EPA], 2012, Human health benchmarks for pesticides: U.S. Environmental Protection Agency, EPA 822-F-12-001, accessed May 19, 2015, at https://nepis.epa.gov/Exe/ZyPDF.cgi/ P100E60P.PDF?Dockey=P100E60P.PDF.

U.S. Environmental Protection Agency [EPA], 2013, Human health benchmarks for pesticides - Updated 2013 technical document: U.S. Environmental Protection Agency, EPA 820-R-13-010, accessed May 19, 2015, at https://nepis.epa.gov/Exe/ZyPDF.cgi/P100GRDJ.PDF? Dockey=P100GRDJ.PDF.

U.S. Geological Survey [USGS], 2003, Principal aquifers of the 48 conterminous United States, Hawaii, Puerto Rico, and the U.S. Virgin Islands: U.S. Geological Survey, digital data, accessed January 4, 2016, at https://water.usgs.gov/ GIS/metadata/usgswrd/XML/aquifers_us.xml.

U.S. Geological Survey [USGS], 2018, USGS water data for the Nation: U.S. Geological Survey National Water Information System database, accessed December 18, 2018, at https://doi.org/10.5066/F7P55KJN.

U.S. Geological Survey [USGS], variously dated, National field manual for the collection of water-quality data: U.S. Geological Survey Techniques of Water-Resources Investigations, book 9, chaps. A1-A9, accessed January 4, 2016, at https://pubs.water.usgs.gov/twri9A.
Vaccaro, J.J., Kahle, S.C., Ely, D.M., Burns, E.R., Snyder, D.T., Haynes, J.V., Olsen, T.D., Welch, W.B., and Morgan, D.S., 2015, Groundwater availability of the Columbia Plateau Regional Aquifer System, Washington, Oregon, and Idaho: U.S. Geological Survey Professional Paper 1817, 87 p., accessed February 2020 at https://doi.org/10.3133/ pp1817.

Wagner, R.J., Boulger, R.W., Jr., Oblinger, C.J., and Smith, B.A., 2006, Guidelines and standard procedures for continuous water-quality monitors - Station operation, record computation, and data reporting: U.S. Geological Survey Techniques and Methods, book 1, chap. D3, 51 p., accessed January 4, 2016, at https:/pubs.usgs.gov/tm/2006/tm1D3/.

Whitehead, R.L., 1992, Geohydrologic framework of the Snake River Plain regional aquifer system, Idaho and eastern Oregon: U.S. Geological Survey Professional Paper 1408-B, 32 p. [Also available at https://doi.org/10.3133/ pp1408B.]

Whitehead, R.L., 1994, Ground water atlas of the United States-Idaho, Oregon, Washington: U.S. Geological Survey Hydrologic Atlas 730-H, accessed January 4, 2016, at https://pubs.usgs.gov/ha/ha730/ch_h/index.html.

Williams, T., Foreman, W.T., Decess, J., Reed-Parker, C., and Stevenson, D.L., 2015, Changes to National Water Quality Laboratory (NWQL) procedures used to establish and verify laboratory detection and reporting limits: U.S. Geological Survey, National Water Quality Laboratory Technical Memorandum 15.02, 21 p., accessed January 4, 2016, at https://nwql.usgs.gov/Public/tech_memos/ nwql.2015-02.pdf.

Zagorsky, J.S., Carter, J.M., Ivahnenko, T., Lapham, W.W., Moran, M.J., Rowe, B.L., Squillace, P.J., and Toccalino, P.L., 2006, The quality of our Nation's waters-Volatile organic compounds in the Nation's ground water and drinking-water supply wells: U.S. Geological Survey Circular 1292, 101 p., accessed January 4, 2016, at https://pubs.usgs.gov/circ/circ1292/. 
Table 1. Information about wells that have environmental data included in this report.

[NAWQA, National Water-Quality Assessment; ID, identification; no., number; LS, land surface; $\mathrm{ft}$ bls, foot below land surface; lat., latitude, in degrees and minutes; long., longitude in degrees and minutes; PAS, principal aquifer study; FL, Florida; na, not available; NGVD 29, National Geodetic Vertical Datum of 1929; NAVD 88, North American Vertical Datum of 1988; ETN, enhanced trends network; TX,

Texas; NM, New Mexico; LUS, land-use study; SC, South Carolina; FPS, flow-path study; MS, Mississippi; VFPS; vertical flow-path study; TN, Tennessee; OK, Oklahoma; AR, Arkansas; CA, California; MO, Missouri; KS, Kansas; MD, Maryland; MSS, modeling support study; DE, Delaware; DC, District of Columbia; MAS, major aquifer study; NV, Nevada; NY, New York; OH, Ohio; MI, Michigan; ID, Idaho;

NH, New Hampshire; WI, Wisconsin; OR, Oregon; MN, Minnesota; WA, Washington]

\begin{tabular}{|c|c|c|c|c|c|c|c|c|c|c|c|c|}
\hline \multirow{2}{*}{$\begin{array}{l}\text { Principal and } \\
\text { regional and (or) } \\
\text { other aquifer } \\
\text { information }\end{array}$} & \multirow{2}{*}{$\begin{array}{l}\text { Network } \\
\text { type }\end{array}$} & \multirow{2}{*}{$\begin{array}{l}\text { Network } \\
\text { name }\end{array}$} & \multirow{2}{*}{$\begin{array}{l}\text { NAWQA well } \\
\text { ID no. }\end{array}$} & \multirow{2}{*}{ State } & \multirow{2}{*}{ County } & \multirow{2}{*}{ Sample date } & \multirow{2}{*}{$\begin{array}{l}\text { Altitude } \\
\text { LS }\end{array}$} & \multirow{2}{*}{$\begin{array}{l}\text { Altitude } \\
\text { datum }\end{array}$} & \multirow{2}{*}{ Water use } & \multirow{2}{*}{$\begin{array}{c}\text { Well } \\
\text { depth } \\
\text { (ft bls) }\end{array}$} & \multicolumn{2}{|c|}{$\begin{array}{c}\text { Depth to perforation } \\
\text { (ft bls) }\end{array}$} \\
\hline & & & & & & & & & & & Top & Bottom \\
\hline $\begin{array}{l}\text { Basin and Range } \\
\text { aquifers }\end{array}$ & MAS & nvbrsus2 & NVBRSUS2-01 & NV & Washoe & $7 / 5 / 2016$ & 4,690 & NGVD 29 & Public supply & 530 & 260 & 520 \\
\hline $\begin{array}{l}\text { Basin and Range } \\
\text { aquifers }\end{array}$ & MAS & nvbrsus2 & NVBRSUS2-02 & NV & Washoe & $7 / 5 / 2016$ & 5,193 & NGVD 29 & Public supply & 760 & 400 & 750 \\
\hline $\begin{array}{l}\text { Basin and Range } \\
\text { aquifers }\end{array}$ & MAS & nvbrsus2 & NVBRSUS2-03 & NV & Washoe & $7 / 6 / 2016$ & 4,700 & NGVD 29 & Domestic & 242 & 109 & 243 \\
\hline $\begin{array}{l}\text { Basin and Range } \\
\text { aquifers }\end{array}$ & MAS & nvbrsus2 & NVBRSUS2-04 & NV & Carson City & 7/7/2016 & $4,620.00$ & NGVD 29 & Observation & 105 & 85 & 105 \\
\hline $\begin{array}{l}\text { Basin and Range } \\
\text { aquifers }\end{array}$ & MAS & nvbrsus2 & NVBRSUS2-05 & NV & Washoe & $7 / 18 / 2016$ & 4,490 & NGVD 29 & Public supply & 429 & 130 & 408 \\
\hline $\begin{array}{l}\text { Basin and Range } \\
\text { aquifers }\end{array}$ & MAS & nvbrsus2 & NVBRSUS2-06 & NV & Washoe & $7 / 19 / 2016$ & 4,480 & NGVD 29 & Public supply & 286 & 110 & 272 \\
\hline $\begin{array}{l}\text { Basin and Range } \\
\text { aquifers }\end{array}$ & MAS & nvbrsus2 & NVBRSUS2-07 & NV & Washoe & $7 / 20 / 2016$ & 4,458 & NGVD 29 & Public supply & 323 & 114 & 308 \\
\hline $\begin{array}{l}\text { Basin and Range } \\
\text { aquifers }\end{array}$ & MAS & nvbrsus2 & NVBRSUS2-08 & NV & Carson City & $7 / 21 / 2016$ & $4,889.10$ & NGVD 29 & Observation & 190 & 175 & 185 \\
\hline $\begin{array}{l}\text { Basin and Range } \\
\text { aquifers }\end{array}$ & MAS & nvbrsus2 & NVBRSUS2-09 & NV & Carson City & $7 / 25 / 2016$ & $4,666.70$ & NGVD 29 & Public supply & 460 & 175 & 450 \\
\hline $\begin{array}{l}\text { Basin and Range } \\
\text { aquifers }\end{array}$ & MAS & nvbrsus2 & NVBRSUS2-10 & NV & Carson City & $7 / 26 / 2016$ & 4,652 & NGVD 29 & Public supply & 700 & 250 & 680 \\
\hline $\begin{array}{l}\text { Basin and Range } \\
\text { aquifers }\end{array}$ & MAS & nvbrsus2 & NVBRSUS2-11 & NV & Washoe & $7 / 27 / 2016$ & 4,485 & NGVD 29 & Public supply & 815 & 238 & 812 \\
\hline $\begin{array}{l}\text { Basin and Range } \\
\text { aquifers }\end{array}$ & MAS & nvbrsus2 & NVBRSUS2-12 & NV & Washoe & $7 / 28 / 2016$ & 4,504 & NGVD 29 & Public supply & 797 & 459 & 797 \\
\hline $\begin{array}{l}\text { Basin and Range } \\
\text { aquifers }\end{array}$ & MAS & nvbrsus2 & NVBRSUS2-13 & NV & Washoe & $8 / 1 / 2016$ & $4,391.60$ & NAVD 88 & Observation & 161 & 151 & 161 \\
\hline $\begin{array}{l}\text { Basin and Range } \\
\text { aquifers }\end{array}$ & MAS & nvbrsus2 & NVBRSUS2-14 & NV & Carson City & $8 / 2 / 2016$ & 4,718 & NGVD 29 & Domestic & 174 & 131 & 174 \\
\hline $\begin{array}{l}\text { Basin and Range } \\
\text { aquifers }\end{array}$ & MAS & nvbrsus2 & NVBRSUS2-15 & NV & Washoe & $8 / 16 / 2016$ & 4,530 & NGVD 29 & Public supply & 334 & 133 & 321 \\
\hline
\end{tabular}


Table 1. Information about wells that have environmental data included in this report.-Continued

[NAWQA, National Water-Quality Assessment; ID, identification; no., number; LS, land surface; ft bls, foot below land surface; lat., latitude, in degrees and minutes; long., longitude in degrees and minutes; PAS, principal aquifer study; FL, Florida; na, not available; NGVD 29, National Geodetic Vertical Datum of 1929; NAVD 88, North American Vertical Datum of 1988; ETN, enhanced trends network; TX, Texas; NM, New Mexico; LUS, land-use study; SC, South Carolina; FPS, flow-path study; MS, Mississippi; VFPS; vertical flow-path study; TN, Tennessee; OK, Oklahoma; AR, Arkansas; CA, California; MO, Missouri; KS, Kansas; MD, Maryland; MSS, modeling support study; DE, Delaware; DC, District of Columbia; MAS, major aquifer study; NV, Nevada; NY, New York; OH, Ohio; MI, Michigan; ID, Idaho; NH, New Hampshire; WI, Wisconsin; OR, Oregon; MN, Minnesota; WA, Washington]

\begin{tabular}{|c|c|c|c|c|c|c|c|c|c|c|c|c|}
\hline \multirow{2}{*}{$\begin{array}{l}\text { Principal and } \\
\text { regional and (or) } \\
\text { other aquifer } \\
\text { information }\end{array}$} & \multirow{2}{*}{$\begin{array}{l}\text { Network } \\
\text { type }\end{array}$} & \multirow{2}{*}{$\begin{array}{l}\text { Network } \\
\text { name }\end{array}$} & \multirow{2}{*}{$\begin{array}{l}\text { NAWQA well } \\
\text { ID no. }\end{array}$} & \multirow{2}{*}{ State } & \multirow{2}{*}{ County } & \multirow{2}{*}{ Sample date } & \multirow{2}{*}{$\begin{array}{l}\text { Altitude } \\
\text { LS }\end{array}$} & \multirow{2}{*}{$\begin{array}{c}\text { Altitude } \\
\text { datum }\end{array}$} & \multirow{2}{*}{ Water use } & \multirow{2}{*}{$\begin{array}{l}\text { Well } \\
\text { depth } \\
\text { (ft bls) }\end{array}$} & \multicolumn{2}{|c|}{$\begin{array}{c}\text { Depth to perforation } \\
\text { (ft bls) }\end{array}$} \\
\hline & & & & & & & & & & & Top & Bottom \\
\hline $\begin{array}{l}\text { Basin and Range } \\
\text { aquifers }\end{array}$ & MAS & nvbrsus2 & NVBRSUS2-16 & NV & Washoe & $8 / 16 / 2016$ & 4,400 & NGVD 29 & Public supply & 191 & 105 & 191 \\
\hline $\begin{array}{l}\text { Basin and Range } \\
\text { aquifers }\end{array}$ & MAS & nvbrsus2 & NVBRSUS2-17 & NV & Washoe & $8 / 17 / 2016$ & 5,800 & NGVD 29 & Public supply & 236 & 173 & 236 \\
\hline $\begin{array}{l}\text { Basin and Range } \\
\text { aquifers }\end{array}$ & MAS & nvbrsus2 & NVBRSUS2-18 & NV & Carson City & $8 / 18 / 2016$ & $4,803.90$ & NGVD 29 & Observation & 238 & 225 & 235 \\
\hline $\begin{array}{l}\text { Basin and Range } \\
\text { aquifers }\end{array}$ & MAS & nvbrsus2 & NVBRSUS2-19 & NV & Washoe & $8 / 22 / 2016$ & 4,500 & NAVD 88 & Observation & 780 & 100 & 770 \\
\hline $\begin{array}{l}\text { Basin and Range } \\
\text { aquifers }\end{array}$ & MAS & nvbrsus2 & NVBRSUS2-20 & NV & Washoe & $8 / 23 / 2016$ & 4,410 & NGVD 29 & Public supply & 274 & 110 & 260 \\
\hline $\begin{array}{l}\text { Basin and Range } \\
\text { aquifers }\end{array}$ & MAS & nvbrsus2 & NVBRSUS2-21 & NV & Washoe & $8 / 23 / 2016$ & 4,438 & NGVD 29 & Public supply & 375 & 143 & 360 \\
\hline $\begin{array}{l}\text { Basin and Range } \\
\text { aquifers }\end{array}$ & MAS & nvbrsus 2 & NVBRSUS2-22 & NV & Washoe & 9/7/2016 & 4,488 & NAVD 88 & Other & 153 & 103 & 153 \\
\hline $\begin{array}{l}\text { Basin and Range } \\
\text { aquifers }\end{array}$ & MAS & nvbrsus2 & NVBRSUS2-23 & NV & Carson City & $9 / 8 / 2016$ & $5,181.50$ & NGVD 29 & Observation & 163 & 148 & 158 \\
\hline $\begin{array}{l}\text { Basin and Range } \\
\text { aquifers }\end{array}$ & MAS & nvbrsus2 & NVBRSUS2-24 & NV & Washoe & $9 / 12 / 2016$ & 4,550 & NGVD 29 & Public supply & 485 & 215 & 475 \\
\hline $\begin{array}{l}\text { Basin and Range } \\
\text { aquifers }\end{array}$ & MAS & nvbrsus2 & NVBRSUS2-25 & NV & Washoe & $9 / 13 / 2016$ & 4,510 & NGVD 29 & Public supply & 456 & 180 & 420 \\
\hline $\begin{array}{l}\text { Basin and Range } \\
\text { aquifers }\end{array}$ & MAS & nvbrsus2 & NVBRSUS2-26 & NV & Washoe & $9 / 13 / 2016$ & 4,563 & NGVD 29 & Public supply & 360 & 170 & 350 \\
\hline $\begin{array}{l}\text { Basin and Range } \\
\text { aquifers }\end{array}$ & MAS & nvbrsus2 & NVBRSUS2-27 & NV & Washoe & $9 / 14 / 2016$ & 4,475 & NGVD 29 & Public supply & 583 & 203 & 441 \\
\hline $\begin{array}{l}\text { Basin and Range } \\
\text { aquifers }\end{array}$ & MAS & nvbrsus2 & NVBRSUS2-28 & NV & Washoe & $9 / 14 / 2016$ & 4,420 & NGVD 29 & Public supply & 665 & 453 & 645 \\
\hline $\begin{array}{l}\text { Basin and Range } \\
\text { aquifers }\end{array}$ & MAS & nvbrsus2 & NVBRSUS2-29 & NV & Washoe & $9 / 15 / 2016$ & 4,410 & NGVD 29 & Public supply & 685 & 330 & 665 \\
\hline $\begin{array}{l}\text { Basin and Range } \\
\text { aquifers }\end{array}$ & MAS & nvbrsus2 & NVBRSUS2-30 & NV & Washoe & $9 / 21 / 2016$ & 4,495 & NGVD 29 & Public supply & 300 & 58 & 288 \\
\hline
\end{tabular}
aquifers 
Table 1. Information about wells that have environmental data included in this report.-Continued

[NAWQA, National Water-Quality Assessment; ID, identification; no., number; LS, land surface; ft bls, foot below land surface; lat., latitude, in degrees and minutes; long., longitude in degrees and minutes; PAS, principal aquifer study; FL, Florida; na, not available; NGVD 29, National Geodetic Vertical Datum of 1929; NAVD 88, North American Vertical Datum of 1988; ETN, enhanced trends network; TX,

Texas; NM, New Mexico; LUS, land-use study; SC, South Carolina; FPS, flow-path study; MS, Mississippi; VFPS; vertical flow-path study; TN, Tennessee; OK, Oklahoma; AR, Arkansas; CA, California; MO, Missouri; KS, Kansas; MD, Maryland; MSS, modeling support study; DE, Delaware; DC, District of Columbia; MAS, major aquifer study; NV, Nevada; NY, New York; OH, Ohio; MI, Michigan; ID, Idaho;

NH, New Hampshire; WI, Wisconsin; OR, Oregon; MN, Minnesota; WA, Washington]

\begin{tabular}{|c|c|c|c|c|c|c|c|c|c|c|c|c|}
\hline \multirow{2}{*}{$\begin{array}{l}\text { Principal and } \\
\text { regional and (or) } \\
\text { other aquifer } \\
\text { information }\end{array}$} & \multirow{2}{*}{$\begin{array}{l}\text { Network } \\
\text { type }\end{array}$} & \multirow{2}{*}{$\begin{array}{l}\text { Network } \\
\text { name }\end{array}$} & \multirow{2}{*}{$\begin{array}{l}\text { NAWQA well } \\
\text { ID no. }\end{array}$} & \multirow{2}{*}{ State } & \multirow{2}{*}{ County } & \multirow{2}{*}{ Sample date } & \multirow{2}{*}{$\begin{array}{l}\text { Altitude } \\
\text { LS }\end{array}$} & \multirow{2}{*}{$\begin{array}{l}\text { Altitude } \\
\text { datum }\end{array}$} & \multirow{2}{*}{ Water use } & \multirow{2}{*}{$\begin{array}{c}\text { Well } \\
\text { depth } \\
\text { (ft bls) }\end{array}$} & \multicolumn{2}{|c|}{$\begin{array}{c}\text { Depth to perforation } \\
\text { (ft bls) }\end{array}$} \\
\hline & & & & & & & & & & & Top & Bottom \\
\hline Biscayne aquifer & PAS & biscpas 1 & BISCPAS1-01 & FL & Palm Beach & $8 / 22 / 2016$ & 15 & NAVD 88 & Public supply & 115 & 105 & 115 \\
\hline Biscayne aquifer & PAS & biscpas1 & BISCPAS1-02 & FL & Palm Beach & $8 / 22 / 2016$ & na & na & Public supply & 77 & na & 77 a \\
\hline Biscayne aquifer & PAS & biscpas 1 & BISCPAS1-03 & FL & Broward & $8 / 23 / 2016$ & na & na & Public supply & 96 & 60 & 96 \\
\hline Biscayne aquifer & PAS & biscpas 1 & BISCPAS1-04 & FL & Broward & $8 / 30 / 2016$ & na & na & Public supply & na & na & na \\
\hline Biscayne aquifer & PAS & biscpas 1 & BISCPAS1-05 & FL & Broward & $6 / 20 / 2016$ & na & na & Public supply & 101 & 75 & 101 \\
\hline Biscayne aquifer & PAS & biscpas 1 & BISCPAS1-06 & FL & Broward & $6 / 20 / 2016$ & na & na & Public supply & 140 & 77 & 140 \\
\hline Biscayne aquifer & PAS & biscpas 1 & BISCPAS1-07 & FL & Broward & $8 / 30 / 2016$ & na & na & Public supply & 152 & 85 & 145 \\
\hline Biscayne aquifer & PAS & biscpas 1 & BISCPAS1-08 & FL & Broward & $6 / 21 / 2016$ & 14 & NGVD 29 & Public supply & 140 & na & $140^{\mathrm{a}}$ \\
\hline Biscayne aquifer & PAS & biscpas 1 & BISCPAS1-09 & FL & Broward & $6 / 21 / 2016$ & na & na & Public supply & 105 & 65 & 105 \\
\hline Biscayne aquifer & PAS & biscpas 1 & BISCPAS1-10 & FL & Broward & $9 / 1 / 2016$ & na & na & Public supply & na & na & na \\
\hline Biscayne aquifer & PAS & biscpas 1 & BISCPAS1-11 & FL & Broward & $6 / 22 / 2016$ & 14.7 & NGVD 29 & Public supply & 115 & 108 & 115 \\
\hline Biscayne aquifer & PAS & biscpas 1 & BISCPAS1-12 & FL & Broward & $6 / 23 / 2016$ & na & na & Public supply & na & na & na \\
\hline Biscayne aquifer & PAS & biscpas 1 & BISCPAS1-13 & FL & Broward & $6 / 22 / 2016$ & 8 & NGVD 29 & Public supply & 90 & 84 & 90 \\
\hline Biscayne aquifer & PAS & biscpas 1 & BISCPAS1-14 & FL & Broward & $8 / 23 / 2016$ & 8 & NGVD 29 & Observation & 22 & 12 & 22 \\
\hline Biscayne aquifer & PAS & biscpas 1 & BISCPAS1-15 & FL & Broward & $6 / 22 / 2016$ & na & na & Public supply & 140 & 75 & 140 \\
\hline Biscayne aquifer & PAS & biscpas 1 & BISCPAS1-16 & FL & Broward & $8 / 29 / 2016$ & na & na & Public supply & 70 & na & $70^{\mathrm{a}}$ \\
\hline Biscayne aquifer & PAS & biscpas 1 & BISCPAS1-17 & FL & Broward & $6 / 23 / 2016$ & na & na & Public supply & 125 & 117 & 125 \\
\hline Biscayne aquifer & PAS & biscpas 1 & BISCPAS1-18 & FL & Broward & $8 / 29 / 2016$ & na & na & Public supply & 112 & 105 & 112 \\
\hline Biscayne aquifer & PAS & biscpas 1 & BISCPAS1-19 & FL & Broward & $6 / 23 / 2016$ & na & na & Public supply & 60 & na & $60 \mathrm{a}$ \\
\hline Biscayne aquifer & PAS & biscpas 1 & BISCPAS1-20 & FL & Broward & $8 / 24 / 2016$ & na & na & Public supply & na & na & na \\
\hline Biscayne aquifer & PAS & biscpas 1 & BISCPAS1-21 & FL & Broward & $8 / 24 / 2016$ & na & na & Public supply & 110 & 100 & 110 \\
\hline Biscayne aquifer & PAS & biscpas 1 & BISCPAS1-22 & FL & Miami-Dade & $6 / 16 / 2016$ & na & na & Public supply & 60 & na & $60^{\mathrm{a}}$ \\
\hline Biscayne aquifer & PAS & biscpas 1 & BISCPAS1-23 & FL & Miami-Dade & $6 / 16 / 2016$ & na & na & Public supply & 60 & 46 & 60 \\
\hline Biscayne aquifer & PAS & biscpas 1 & BISCPAS1-24 & FL & Miami-Dade & $6 / 15 / 2016$ & 7 & NGVD 29 & Public supply & 95 & 66 & 95 \\
\hline Biscayne aquifer & PAS & biscpas 1 & BISCPAS1-25 & FL & Miami-Dade & $6 / 15 / 2016$ & 12 & NGVD 29 & Public supply & 91 & 85 & 91 \\
\hline Biscayne aquifer & PAS & biscpas 1 & BISCPAS1-26 & FL & Miami-Dade & $6 / 13 / 2016$ & na & na & Observation & 40 & na & $40^{a}$ \\
\hline Biscayne aquifer & PAS & biscpas 1 & BISCPAS1-27 & FL & Miami-Dade & $6 / 14 / 2016$ & 9.5 & NGVD 29 & Public supply & 100 & 45 & 100 \\
\hline
\end{tabular}


Table 1. Information about wells that have environmental data included in this report.-Continued

[NAWQA, National Water-Quality Assessment; ID, identification; no., number; LS, land surface; ft bls, foot below land surface; lat., latitude, in degrees and minutes; long., longitude in degrees and minutes; PAS, principal aquifer study; FL, Florida; na, not available; NGVD 29, National Geodetic Vertical Datum of 1929; NAVD 88, North American Vertical Datum of 1988; ETN, enhanced trends network; TX, Texas; NM, New Mexico; LUS, land-use study; SC, South Carolina; FPS, flow-path study; MS, Mississippi; VFPS; vertical flow-path study; TN, Tennessee; OK, Oklahoma; AR, Arkansas; CA, California; MO, Missouri; KS, Kansas; MD, Maryland; MSS, modeling support study; DE, Delaware; DC, District of Columbia; MAS, major aquifer study; NV, Nevada; NY, New York; OH, Ohio; MI, Michigan; ID, Idaho; NH, New Hampshire; WI, Wisconsin; OR, Oregon; MN, Minnesota; WA, Washington]

\begin{tabular}{|c|c|c|c|c|c|c|c|c|c|c|c|c|}
\hline \multirow{2}{*}{$\begin{array}{l}\text { Principal and } \\
\text { regional and (or) } \\
\text { other aquifer } \\
\text { information }\end{array}$} & \multirow{2}{*}{$\begin{array}{l}\text { Network } \\
\text { type }\end{array}$} & \multirow{2}{*}{$\begin{array}{l}\text { Network } \\
\text { name }\end{array}$} & \multirow{2}{*}{$\begin{array}{l}\text { NAWQA well } \\
\text { ID no. }\end{array}$} & \multirow{2}{*}{ State } & \multirow{2}{*}{ County } & \multirow{2}{*}{ Sample date } & \multirow{2}{*}{$\begin{array}{l}\text { Altitude } \\
\text { LS }\end{array}$} & \multirow{2}{*}{$\begin{array}{l}\text { Altitude } \\
\text { datum }\end{array}$} & \multirow{2}{*}{ Water use } & \multirow{2}{*}{$\begin{array}{l}\text { Well } \\
\text { depth } \\
\text { (ft bls) }\end{array}$} & \multicolumn{2}{|c|}{$\begin{array}{l}\text { Depth to perforation } \\
\text { (ft bls) }\end{array}$} \\
\hline & & & & & & & & & & & Top & Bottom \\
\hline Biscayne aquifer & PAS & biscpas 1 & BISCPAS1-28 & FL & Miami-Dade & $8 / 31 / 2016$ & na & na & Public supply & na & na & na \\
\hline Biscayne aquifer & PAS & biscpas1 & BISCPAS1-29 & FL & Miami-Dade & $6 / 14 / 2016$ & 8.2 & NGVD 29 & Public supply & 132 & 61 & 132 \\
\hline Biscayne aquifer & PAS & biscpas 1 & BISCPAS1-30 & FL & Miami-Dade & $6 / 13 / 2016$ & na & na & Public supply & 90 & 45 & 90 \\
\hline Biscayne aquifer & PAS & biscpas 1 & BISCPAS1-31 & FL & Miami-Dade & $8 / 31 / 2016$ & na & na & Public supply & na & na & na \\
\hline Biscayne aquifer & PAS & biscpas1 & BISCPAS1-32 & FL & Miami-Dade & $6 / 8 / 2016$ & na & na & Public supply & 35 & 27 & 30 \\
\hline Biscayne aquifer & PAS & biscpas 1 & BISCPAS1-33 & FL & Miami-Dade & 6/9/2016 & na & na & Public supply & na & na & na \\
\hline Biscayne aquifer & PAS & biscpas1 & BISCPAS1-34 & FL & Miami-Dade & $6 / 8 / 2016$ & na & na & Public supply & na & na & na \\
\hline Biscayne aquifer & PAS & biscpas1 & BISCPAS1-35 & FL & Miami-Dade & $6 / 8 / 2016$ & na & na & Public supply & 40 & 37 & 40 \\
\hline Biscayne aquifer & PAS & biscpas1 & BISCPAS1-36 & FL & Miami-Dade & $6 / 7 / 2016$ & na & na & Public supply & 61 & 31 & 61 \\
\hline Biscayne aquifer & PAS & biscpas 1 & BISCPAS1-37 & FL & Miami-Dade & $8 / 25 / 2016$ & na & na & Observation & 62 & 40 & 62 \\
\hline Biscayne aquifer & PAS & biscpas1 & BISCPAS1-38 & FL & Miami-Dade & 6/7/2016 & 5.3 & NGVD 29 & Observation & 22 & 17 & 22 \\
\hline Biscayne aquifer & PAS & biscpas 1 & BISCPAS1-39 & FL & Miami-Dade & $6 / 6 / 2016$ & 7 & NGVD 29 & Public supply & 60 & 20 & 60 \\
\hline Biscayne aquifer & PAS & biscpas 1 & BISCPAS1-40 & FL & Miami-Dade & 6/6/2016 & na & na & Public supply & na & na & na \\
\hline $\begin{array}{l}\text { Central Valley } \\
\text { aquifer system }\end{array}$ & FPS & cvalfps 1 & CVALFPS1-B1-1 & $\mathrm{CA}$ & Fresno & $7 / 25 / 2013$ & 353 & NGVD 29 & Observation & 81 & 71 & 76 \\
\hline $\begin{array}{l}\text { Central Valley } \\
\text { aquifer system }\end{array}$ & FPS & cvalfps1 & CVALFPS1-B1-2 & $\mathrm{CA}$ & Fresno & $7 / 25 / 2013$ & 353 & NGVD 29 & Observation & 168 & 158 & 163 \\
\hline $\begin{array}{l}\text { Central Valley } \\
\text { aquifer system }\end{array}$ & FPS & cvalfps 1 & CVALFPS1-B1-3 & $\mathrm{CA}$ & Fresno & $8 / 12 / 2013$ & 353 & NGVD 29 & Observation & 268 & 258 & 263 \\
\hline $\begin{array}{l}\text { Central Valley } \\
\text { aquifer system }\end{array}$ & FPS & cvalfps 1 & CVALFPS1-B2.5-1 & $\mathrm{CA}$ & Fresno & $9 / 25 / 2013$ & 343 & NGVD 29 & Observation & 140 & 130 & 135 \\
\hline $\begin{array}{l}\text { Central Valley } \\
\text { aquifer system }\end{array}$ & FPS & cvalfps 1 & CVALFPS1-B2.5-2 & $\mathrm{CA}$ & Fresno & $9 / 25 / 2013$ & 343 & NGVD 29 & Observation & 177 & 167 & 172 \\
\hline $\begin{array}{l}\text { Central Valley } \\
\text { aquifer system }\end{array}$ & FPS & cvalfps 1 & CVALFPS1-B2-1 & $\mathrm{CA}$ & Fresno & 9/10/2013 & 350 & NGVD 29 & Observation & 81 & 71 & 76 \\
\hline $\begin{array}{l}\text { Central Valley } \\
\text { aquifer system }\end{array}$ & FPS & cvalfps 1 & CVALFPS1-B2-2 & $\mathrm{CA}$ & Fresno & 9/9/2013 & 351 & NGVD 29 & Observation & 89 & 79 & 84 \\
\hline $\begin{array}{l}\text { Central Valley } \\
\text { aquifer system }\end{array}$ & FPS & cvalfps 1 & CVALFPS1-B2-3 & $\mathrm{CA}$ & Fresno & 9/9/2013 & 351 & NGVD 29 & Observation & 135 & 125 & 130 \\
\hline
\end{tabular}


Table 1. Information about wells that have environmental data included in this report.-Continued

[NAWQA, National Water-Quality Assessment; ID, identification; no., number; LS, land surface; $\mathrm{ft}$ bls, foot below land surface; lat., latitude, in degrees and minutes; long., longitude in degrees and minutes; PAS, principal aquifer study; FL, Florida; na, not available; NGVD 29, National Geodetic Vertical Datum of 1929; NAVD 88, North American Vertical Datum of 1988; ETN, enhanced trends network; TX,

Texas; NM, New Mexico; LUS, land-use study; SC, South Carolina; FPS, flow-path study; MS, Mississippi; VFPS; vertical flow-path study; TN, Tennessee; OK, Oklahoma; AR, Arkansas; CA, California; MO, Missouri; KS, Kansas; MD, Maryland; MSS, modeling support study; DE, Delaware; DC, District of Columbia; MAS, major aquifer study; NV, Nevada; NY, New York; OH, Ohio; MI, Michigan; ID, Idaho;

NH, New Hampshire; WI, Wisconsin; OR, Oregon; MN, Minnesota; WA, Washington]

\begin{tabular}{|c|c|c|c|c|c|c|c|c|c|c|c|c|}
\hline \multirow{2}{*}{$\begin{array}{l}\text { Principal and } \\
\text { regional and (or) } \\
\text { other aquifer } \\
\text { information }\end{array}$} & \multirow{2}{*}{$\begin{array}{l}\text { Network } \\
\text { type }\end{array}$} & \multirow{2}{*}{$\begin{array}{l}\text { Network } \\
\text { name }\end{array}$} & \multirow{2}{*}{$\begin{array}{l}\text { NAWQA well } \\
\text { ID no. }\end{array}$} & \multirow{2}{*}{ State } & \multirow{2}{*}{ County } & \multirow{2}{*}{ Sample date } & \multirow{2}{*}{$\begin{array}{c}\text { Altitude } \\
\text { LS }\end{array}$} & \multirow{2}{*}{$\begin{array}{l}\text { Altitude } \\
\text { datum }\end{array}$} & \multirow{2}{*}{ Water use } & \multirow{2}{*}{$\begin{array}{c}\text { Well } \\
\text { depth } \\
\text { (ft bls) }\end{array}$} & \multicolumn{2}{|c|}{$\begin{array}{c}\text { Depth to perforation } \\
\text { (ft bls) }\end{array}$} \\
\hline & & & & & & & & & & & Top & Bottom \\
\hline $\begin{array}{l}\text { Central Valley } \\
\text { aquifer system }\end{array}$ & FPS & cvalfps1 & CVALFPS1-B3-1 & $\mathrm{CA}$ & Fresno & $8 / 13 / 2013$ & 342 & NGVD 29 & Observation & 70 & 60 & 65 \\
\hline $\begin{array}{l}\text { Central Valley } \\
\text { aquifer system }\end{array}$ & FPS & cvalfps1 & CVALFPS1-B3-2 & $\mathrm{CA}$ & Fresno & $8 / 14 / 2013$ & 342 & NGVD 29 & Observation & 113 & 103 & 108 \\
\hline $\begin{array}{l}\text { Central Valley } \\
\text { aquifer system }\end{array}$ & FPS & cvalfps1 & CVALFPS1-B3-3 & $\mathrm{CA}$ & Fresno & $8 / 13 / 2013$ & 342 & NGVD 29 & Observation & 172 & 162 & 167 \\
\hline $\begin{array}{l}\text { Central Valley } \\
\text { aquifer system }\end{array}$ & FPS & cvalfps1 & CVALFPS1-B3-4 & $\mathrm{CA}$ & Fresno & $8 / 14 / 2013$ & 342 & NGVD 29 & Observation & 197 & 187 & 192 \\
\hline $\begin{array}{l}\text { Central Valley } \\
\text { aquifer system }\end{array}$ & FPS & cvalfps 1 & CVALFPS1-B3-5 & $\mathrm{CA}$ & Fresno & $8 / 13 / 2013$ & 342 & NGVD 29 & Observation & 265 & 255 & 260 \\
\hline $\begin{array}{l}\text { Central Valley } \\
\text { aquifer system }\end{array}$ & FPS & cvalfps1 & CVALFPS1-B4-1 & $\mathrm{CA}$ & Fresno & $8 / 19 / 2013$ & 337 & NGVD 29 & Observation & 77 & 67 & 72 \\
\hline $\begin{array}{l}\text { Central Valley } \\
\text { aquifer system }\end{array}$ & FPS & cvalfps 1 & CVALFPS1-B4-2 & $\mathrm{CA}$ & Fresno & $8 / 20 / 2013$ & 337 & NGVD 29 & Observation & 115 & 105 & 110 \\
\hline $\begin{array}{l}\text { Central Valley } \\
\text { aquifer system }\end{array}$ & FPS & cvalfps 1 & CVALFPS1-B4-3 & $\mathrm{CA}$ & Fresno & $8 / 20 / 2013$ & 337 & NGVD 29 & Observation & 184 & 174 & 179 \\
\hline $\begin{array}{l}\text { Central Valley } \\
\text { aquifer system }\end{array}$ & FPS & cvalfps1 & CVALFPS1-B4-4 & $\mathrm{CA}$ & Fresno & $8 / 19 / 2013$ & 337 & NGVD 29 & Observation & 261 & 251 & 256 \\
\hline $\begin{array}{l}\text { Central Valley } \\
\text { aquifer system }\end{array}$ & FPS & cvalfps 1 & CVALFPS1-B5-1 & $\mathrm{CA}$ & Fresno & $7 / 23 / 2013$ & 329 & NGVD 29 & Observation & 80 & 70 & 75 \\
\hline $\begin{array}{l}\text { Central Valley } \\
\text { aquifer system }\end{array}$ & FPS & cvalfps 1 & CVALFPS1-B5-2 & $\mathrm{CA}$ & Fresno & $7 / 23 / 2013$ & 329 & NGVD 29 & Observation & 158 & 148 & 153 \\
\hline $\begin{array}{l}\text { Central Valley } \\
\text { aquifer system }\end{array}$ & FPS & cvalfps1 & CVALFPS1-B5-3 & $\mathrm{CA}$ & Fresno & $7 / 24 / 2013$ & 329 & NGVD 29 & Observation & 268 & 258 & 263 \\
\hline $\begin{array}{l}\text { Central Valley } \\
\text { aquifer system }\end{array}$ & FPS & cvalfps 2 & $\begin{array}{l}\text { CVALFPS2-FPC1- } \\
\text { shallow }\end{array}$ & $\mathrm{CA}$ & Fresno & $7 / 24 / 2013$ & 356 & NGVD 29 & Domestic & 125 & 125 & 125 \\
\hline $\begin{array}{l}\text { Central Valley } \\
\text { aquifer system }\end{array}$ & FPS & cvalfps 2 & $\begin{array}{l}\text { CVALFPS2-FPC2- } \\
\text { deep }\end{array}$ & $\mathrm{CA}$ & Fresno & $8 / 28 / 2013$ & 328 & NAVD 88 & Public supply & 400 & 150 & 390 \\
\hline $\begin{array}{l}\text { Central Valley } \\
\text { aquifer system }\end{array}$ & FPS & cvalfps 2 & $\begin{array}{l}\text { CVALFPS2-FPC2- } \\
\text { shallow }\end{array}$ & $\mathrm{CA}$ & Fresno & $7 / 22 / 2013$ & 331 & NGVD 29 & Domestic & 132 & na & $132^{\mathrm{a}}$ \\
\hline
\end{tabular}


Table 1. Information about wells that have environmental data included in this report.-Continued

[NAWQA, National Water-Quality Assessment; ID, identification; no., number; LS, land surface; ft bls, foot below land surface; lat., latitude, in degrees and minutes; long., longitude in degrees and minutes; PAS, principal aquifer study; FL, Florida; na, not available; NGVD 29, National Geodetic Vertical Datum of 1929; NAVD 88, North American Vertical Datum of 1988; ETN, enhanced trends network; TX, Texas; NM, New Mexico; LUS, land-use study; SC, South Carolina; FPS, flow-path study; MS, Mississippi; VFPS; vertical flow-path study; TN, Tennessee; OK, Oklahoma; AR, Arkansas; CA, California; MO, Missouri; KS, Kansas; MD, Maryland; MSS, modeling support study; DE, Delaware; DC, District of Columbia; MAS, major aquifer study; NV, Nevada; NY, New York; OH, Ohio; MI, Michigan; ID, Idaho; NH, New Hampshire; WI, Wisconsin; OR, Oregon; MN, Minnesota; WA, Washington]

\begin{tabular}{|c|c|c|c|c|c|c|c|c|c|c|c|c|}
\hline \multirow{2}{*}{$\begin{array}{l}\text { Principal and } \\
\text { regional and (or) } \\
\text { other aquifer } \\
\text { information }\end{array}$} & \multirow{2}{*}{$\begin{array}{l}\text { Network } \\
\text { type }\end{array}$} & \multirow{2}{*}{$\begin{array}{c}\text { Network } \\
\text { name }\end{array}$} & \multirow{2}{*}{$\begin{array}{l}\text { NAWQA well } \\
\text { ID no. }\end{array}$} & \multirow{2}{*}{ State } & \multirow{2}{*}{ County } & \multirow{2}{*}{ Sample date } & \multirow{2}{*}{$\begin{array}{c}\text { Altitude } \\
\text { LS }\end{array}$} & \multirow{2}{*}{$\begin{array}{l}\text { Altitude } \\
\text { datum }\end{array}$} & \multirow{2}{*}{ Water use } & \multirow{2}{*}{$\begin{array}{c}\text { Well } \\
\text { depth } \\
\text { (ft bls) }\end{array}$} & \multicolumn{2}{|c|}{$\begin{array}{c}\text { Depth to perforation } \\
\text { (ft bls) }\end{array}$} \\
\hline & & & & & & & & & & & Top & Bottom \\
\hline $\begin{array}{l}\text { Central Valley } \\
\text { aquifer system }\end{array}$ & FPS & cvalfps 2 & $\begin{array}{l}\text { CVALFPS2-FPC3- } \\
\text { shallow }\end{array}$ & $\mathrm{CA}$ & Fresno & 9/17/2013 & 312 & NAVD 88 & Domestic & 145 & 85 & 145 \\
\hline $\begin{array}{l}\text { Central Valley } \\
\text { aquifer system }\end{array}$ & FPS & cvalfps 2 & $\begin{array}{l}\text { CVALFPS2-FPF1- } \\
\text { deep }\end{array}$ & $\mathrm{CA}$ & Fresno & $8 / 27 / 2013$ & 300 & NAVD 88 & Public supply & 530 & 180 & 520 \\
\hline $\begin{array}{l}\text { Central Valley } \\
\text { aquifer system }\end{array}$ & FPS & cvalfps 2 & $\begin{array}{l}\text { CVALFPS2-FPF1- } \\
\text { shallow }\end{array}$ & $\mathrm{CA}$ & Fresno & $9 / 11 / 2013$ & 300 & NAVD 88 & Observation & 160 & 140 & 150 \\
\hline $\begin{array}{l}\text { Central Valley } \\
\text { aquifer system }\end{array}$ & FPS & cvalfps 2 & $\begin{array}{l}\text { CVALFPS2-FPF2- } \\
\text { deep }\end{array}$ & $\mathrm{CA}$ & Fresno & $8 / 27 / 2013$ & 313 & NAVD 88 & Public supply & 455 & 170 & 455 \\
\hline $\begin{array}{l}\text { Central Valley } \\
\text { aquifer system }\end{array}$ & FPS & cvalfps2 & $\begin{array}{l}\text { CVALFPS2-FPF2- } \\
\text { shallow }\end{array}$ & $\mathrm{CA}$ & Fresno & $9 / 11 / 2013$ & 313 & NAVD 88 & Observation & 190 & 170 & 180 \\
\hline $\begin{array}{l}\text { Central Valley } \\
\text { aquifer system }\end{array}$ & FPS & cvalfps 2 & $\begin{array}{l}\text { CVALFPS2-FPR1- } \\
\text { deep }\end{array}$ & $\mathrm{CA}$ & Fresno & $8 / 28 / 2013$ & 298 & NAVD 88 & Public supply & 440 & 230 & 430 \\
\hline $\begin{array}{l}\text { Central Valley } \\
\text { aquifer system }\end{array}$ & FPS & cvalfps2 & $\begin{array}{l}\text { CVALFPS2-FPR1- } \\
\text { shallow }\end{array}$ & $\mathrm{CA}$ & Fresno & $9 / 24 / 2013$ & 295 & NAVD 88 & Domestic & 100 & 80 & 100 \\
\hline $\begin{array}{l}\text { Central Valley } \\
\text { aquifer system }\end{array}$ & FPS & cvalfps 2 & $\begin{array}{l}\text { CVALFPS2-FPR2- } \\
\text { deep }\end{array}$ & $\mathrm{CA}$ & Fresno & $9 / 19 / 2013$ & 275 & NAVD 88 & Public supply & 370.3 & 370.3 & 370.3 \\
\hline $\begin{array}{l}\text { Central Valley } \\
\text { aquifer system }\end{array}$ & FPS & cvalfps 2 & $\begin{array}{l}\text { CVALFPS2-FPR2- } \\
\text { shallow }\end{array}$ & $\mathrm{CA}$ & Fresno & $8 / 22 / 2013$ & 261 & NAVD 88 & Domestic & 180 & 140 & 180 \\
\hline $\begin{array}{l}\text { Central Valley } \\
\text { aquifer system }\end{array}$ & FPS & cvalfps 2 & $\begin{array}{l}\text { CVALFPS2-FPR3- } \\
\text { deep }\end{array}$ & $\mathrm{CA}$ & Fresno & $9 / 18 / 2013$ & 232 & NAVD 88 & Other & 504 & 210 & 504 \\
\hline $\begin{array}{l}\text { Central Valley } \\
\text { aquifer system }\end{array}$ & FPS & cvalfps 2 & $\begin{array}{l}\text { CVALFPS2-FPR3- } \\
\text { shallow }\end{array}$ & $\mathrm{CA}$ & Fresno & $8 / 29 / 2013$ & 231 & NAVD 88 & Public supply & 245 & 185 & 245 \\
\hline $\begin{array}{l}\text { Central Valley } \\
\text { aquifer system }\end{array}$ & FPS & cvalfps 2 & $\begin{array}{l}\text { CVALFPS2-FPR4- } \\
\text { deep }\end{array}$ & $\mathrm{CA}$ & Fresno & $8 / 26 / 2013$ & 214 & NAVD 88 & Other & 500 & 335 & 490 \\
\hline $\begin{array}{l}\text { Central Valley } \\
\text { aquifer system }\end{array}$ & FPS & cvalfps2 & $\begin{array}{l}\text { CVALFPS2-FPR4- } \\
\text { shallow }\end{array}$ & $\mathrm{CA}$ & Fresno & $9 / 18 / 2013$ & 213 & NAVD 88 & Domestic & 212 & 152 & 212 \\
\hline $\begin{array}{l}\text { Central Valley } \\
\text { aquifer system }\end{array}$ & FPS & cvalfps 2 & $\begin{array}{l}\text { CVALFPS2-FPR5- } \\
\text { deep }\end{array}$ & $\mathrm{CA}$ & Fresno & $8 / 26 / 2013$ & 193 & NAVD 88 & Other & 532 & 250 & 528 \\
\hline $\begin{array}{l}\text { Central Valley } \\
\text { aquifer system }\end{array}$ & FPS & cvalfps2 & $\begin{array}{l}\text { CVALFPS2-FPR5- } \\
\text { shallow }\end{array}$ & $\mathrm{CA}$ & Fresno & $9 / 12 / 2013$ & 205 & NAVD 88 & Domestic & 300 & 300 & 300 \\
\hline
\end{tabular}


Table 1. Information about wells that have environmental data included in this report.-Continued

[NAWQA, National Water-Quality Assessment; ID, identification; no., number; LS, land surface; ft bls, foot below land surface; lat., latitude, in degrees and minutes; long., longitude in degrees and minutes; PAS, principal aquifer study; FL, Florida; na, not available; NGVD 29, National Geodetic Vertical Datum of 1929; NAVD 88, North American Vertical Datum of 1988; ETN, enhanced trends network; TX,

Texas; NM, New Mexico; LUS, land-use study; SC, South Carolina; FPS, flow-path study; MS, Mississippi; VFPS; vertical flow-path study; TN, Tennessee; OK, Oklahoma; AR, Arkansas; CA, California; MO, Missouri; KS, Kansas; MD, Maryland; MSS, modeling support study; DE, Delaware; DC, District of Columbia; MAS, major aquifer study; NV, Nevada; NY, New York; OH, Ohio; MI, Michigan; ID, Idaho;

NH, New Hampshire; WI, Wisconsin; OR, Oregon; MN, Minnesota; WA, Washington]

\begin{tabular}{|c|c|c|c|c|c|c|c|c|c|c|c|c|}
\hline \multirow{2}{*}{$\begin{array}{l}\text { Principal and } \\
\text { regional and (or) } \\
\text { other aquifer } \\
\text { information }\end{array}$} & \multirow{2}{*}{$\begin{array}{l}\text { Network } \\
\text { type }\end{array}$} & \multirow{2}{*}{$\begin{array}{l}\text { Network } \\
\text { name }\end{array}$} & \multirow{2}{*}{$\begin{array}{l}\text { NAWOA well } \\
\text { ID no. }\end{array}$} & \multirow{2}{*}{ State } & \multirow{2}{*}{ County } & \multirow{2}{*}{ Sample date } & \multirow{2}{*}{$\begin{array}{l}\text { Altitude } \\
\text { LS }\end{array}$} & \multirow{2}{*}{$\begin{array}{l}\text { Altitude } \\
\text { datum }\end{array}$} & \multirow{2}{*}{ Water use } & \multirow{2}{*}{$\begin{array}{c}\text { Well } \\
\text { depth } \\
\text { (ft bls) }\end{array}$} & \multicolumn{2}{|c|}{$\begin{array}{c}\text { Depth to perforation } \\
\text { (ft bls) }\end{array}$} \\
\hline & & & & & & & & & & & Top & Bottom \\
\hline $\begin{array}{l}\text { Central Valley } \\
\text { aquifer system }\end{array}$ & FPS & cvalfps2 & $\begin{array}{l}\text { CVALFPS2-FPR6- } \\
\text { deep }\end{array}$ & $\mathrm{CA}$ & Fresno & $8 / 21 / 2013$ & 170 & NGVD 29 & Other & 520 & 280 & 520 \\
\hline $\begin{array}{l}\text { Central Valley } \\
\text { aquifer system }\end{array}$ & FPS & cvalfps 2 & $\begin{array}{l}\text { CVALFPS2-FPR6- } \\
\text { shallow }\end{array}$ & $\mathrm{CA}$ & Fresno & $9 / 16 / 2013$ & 188 & NAVD 88 & Domestic & 300 & 240 & 300 \\
\hline $\begin{array}{l}\text { Central Valley } \\
\text { aquifer system }\end{array}$ & ETN & cvaletn1 & CVALETN1-01 & $\mathrm{CA}$ & Fresno & $\begin{array}{l}3 / 3,3 / 17 \\
5 / 17,11 / 17 \\
12 / 7 / 2016\end{array}$ & 308 & NAVD 88 & Public supply & 620 & 410 & 610 \\
\hline $\begin{array}{l}\text { Central Valley } \\
\text { aquifer system }\end{array}$ & $\begin{array}{l}\text { ETN/ } \\
\text { FPS }\end{array}$ & $\begin{array}{l}\text { cvaletn1/ } \\
\text { cvalfps2 }\end{array}$ & $\begin{array}{l}\text { CVALETN1-02/ } \\
\text { CVALFPS2-FPC3- } \\
\text { deep }\end{array}$ & $\mathrm{CA}$ & Fresno & $\begin{array}{l}9 / 17 / 2013 \\
\quad(\mathrm{fps} 2) ; 3 / 3 \\
7 / 17 / 2016\end{array}$ & 308 & NAVD 88 & Public supply & 320 & 160 & 310 \\
\hline $\begin{array}{l}\text { Central Valley } \\
\text { aquifer system }\end{array}$ & ETN & cvaletn 1 & CVALETN1-03 & $\mathrm{CA}$ & Fresno & $\begin{array}{c}3 / 17,5 / 17,8 / 24 \\
12 / 7 / 2016\end{array}$ & 308 & NAVD 88 & Observation & 234 & 214 & 224 \\
\hline $\begin{array}{l}\text { Columbia } \\
\text { Plateau } \\
\text { basin-fill and } \\
\text { basaltic-rock } \\
\text { aquifers }\end{array}$ & ETN & clptetn1 & CLPTETN1-01 & OR & Morrow & $\begin{array}{l}1 / 20,3 / 18 \\
4 / 21,6 / 22 \\
7 / 28,9 / 8 \\
11 / 17 / 2016\end{array}$ & 292 & NGVD 29 & Domestic & 80 & 79 & 80 \\
\hline $\begin{array}{l}\text { Columbia } \\
\text { Plateau } \\
\text { basin-fill and } \\
\text { basaltic-rock } \\
\text { aquifers }\end{array}$ & ETN & clptetn 1 & CLPTETN1-05 & OR & Umatilla & $\begin{array}{c}3 / 17,4 / 21,6 / 23 \\
9 / 9,10 / 25 \\
11 / 18 / 2016\end{array}$ & 616 & NAVD 88 & Other & 170 & 144 & 170 \\
\hline $\begin{array}{l}\text { Columbia } \\
\text { Plateau } \\
\text { basaltic-rock } \\
\text { aquifers }\end{array}$ & PAS & clptpas1 & CLPTPAS1-01 & WA & Whitman & $8 / 4 / 2016$ & $1,557.90$ & NAVD 88 & Public supply & 273 & 86 & 273 \\
\hline $\begin{array}{l}\text { Columbia } \\
\text { Plateau } \\
\text { basaltic-rock } \\
\text { aquifers }\end{array}$ & PAS & clptpas1 & CLPTPAS1-02 & WA & Yakima & $6 / 27 / 2016$ & 1,110 & NGVD 29 & Public supply & 1,171 & 878 & 1,163 \\
\hline
\end{tabular}


Table 1. Information about wells that have environmental data included in this report.-Continued

[NAWQA, National Water-Quality Assessment; ID, identification; no., number; LS, land surface; ft bls, foot below land surface; lat., latitude, in degrees and minutes; long., longitude in degrees and minutes; PAS, principal aquifer study; FL, Florida; na, not available; NGVD 29, National Geodetic Vertical Datum of 1929; NAVD 88, North American Vertical Datum of 1988; ETN, enhanced trends network; TX, Texas; NM, New Mexico; LUS, land-use study; SC, South Carolina; FPS, flow-path study; MS, Mississippi; VFPS; vertical flow-path study; TN, Tennessee; OK, Oklahoma; AR, Arkansas; CA, California; MO, Missouri; KS, Kansas; MD, Maryland; MSS, modeling support study; DE, Delaware; DC, District of Columbia; MAS, major aquifer study; NV, Nevada; NY, New York; OH, Ohio; MI, Michigan; ID, Idaho; NH, New Hampshire; WI, Wisconsin; OR, Oregon; MN, Minnesota; WA, Washington]

\begin{tabular}{|c|c|c|c|c|c|c|c|c|c|c|c|c|}
\hline \multirow{2}{*}{$\begin{array}{l}\text { Principal and } \\
\text { regional and (or) } \\
\text { other aquifer } \\
\text { information }\end{array}$} & \multirow{2}{*}{$\begin{array}{l}\text { Network } \\
\text { type }\end{array}$} & \multirow{2}{*}{$\begin{array}{l}\text { Network } \\
\text { name }\end{array}$} & \multirow{2}{*}{$\begin{array}{l}\text { NAWOA well } \\
\text { ID no. }\end{array}$} & \multirow{2}{*}{ State } & \multirow{2}{*}{ County } & \multirow{2}{*}{ Sample date } & \multirow{2}{*}{$\begin{array}{l}\text { Altitude } \\
\text { LS }\end{array}$} & \multirow{2}{*}{$\begin{array}{l}\text { Altitude } \\
\text { datum }\end{array}$} & \multirow{2}{*}{ Water use } & \multirow{2}{*}{$\begin{array}{c}\text { Well } \\
\text { depth } \\
\text { (ft bls) }\end{array}$} & \multicolumn{2}{|c|}{$\begin{array}{c}\text { Depth to perforation } \\
\text { (ft bls) }\end{array}$} \\
\hline & & & & & & & & & & & Top & Bottom \\
\hline $\begin{array}{l}\text { Columbia } \\
\text { Plateau } \\
\text { basaltic-rock } \\
\text { aquifers }\end{array}$ & PAS & clptpas 1 & CLPTPAS1-03 & WA & Grant & $6 / 28 / 2016$ & 533 & NAVD 88 & Public supply & 91 & 86 & 91 \\
\hline $\begin{array}{l}\text { Columbia } \\
\text { Plateau } \\
\text { basaltic-rock } \\
\text { aquifers }\end{array}$ & PAS & clptpas1 & CLPTPAS1-04 & WA & Kittitas & $6 / 29 / 2016$ & 1,570 & NAVD 88 & Public supply & na & na & na \\
\hline $\begin{array}{l}\text { Columbia } \\
\text { Plateau } \\
\text { basaltic-rock } \\
\text { aquifers }\end{array}$ & PAS & clptpas1 & CLPTPAS1-05 & WA & Kittitas & $6 / 30 / 2016$ & 1,625 & NAVD 88 & Public supply & 720 & 453 & 720 \\
\hline $\begin{array}{l}\text { Columbia } \\
\text { Plateau } \\
\text { basaltic-rock } \\
\text { aquifers }\end{array}$ & PAS & clptpas1 & CLPTPAS1-06 & WA & Kittitas & $6 / 30 / 2016$ & 2,240 & NGVD 29 & Public supply & 500 & 190 & 500 \\
\hline $\begin{array}{l}\text { Columbia } \\
\text { Plateau } \\
\text { basaltic-rock } \\
\text { aquifers }\end{array}$ & PAS & clptpas1 & CLPTPAS1-07 & WA & Adams & $7 / 11 / 2016$ & 931 & NAVD 88 & Public supply & 280 & na & $280^{\mathrm{a}}$ \\
\hline $\begin{array}{l}\text { Columbia } \\
\text { Plateau } \\
\text { basaltic-rock } \\
\text { aquifers }\end{array}$ & PAS & clptpas1 & CLPTPAS1-08 & WA & Grant & $7 / 11 / 2016$ & 1,129 & NAVD 88 & Public supply & 335 & 26 & 335 \\
\hline $\begin{array}{l}\text { Columbia } \\
\text { Plateau } \\
\text { basaltic-rock } \\
\text { aquifers }\end{array}$ & PAS & clptpas1 & CLPTPAS1-09 & WA & Franklin & $7 / 12 / 2016$ & 860 & NGVD 29 & Public supply & 1,325 & 750 & 1,325 \\
\hline $\begin{array}{l}\text { Columbia } \\
\text { Plateau } \\
\text { basaltic-rock } \\
\text { aquifers }\end{array}$ & PAS & clptpas1 & CLPTPAS1-10 & WA & Benton & $7 / 13 / 2016$ & na & na & Public supply & na & na & na \\
\hline
\end{tabular}


Table 1. Information about wells that have environmental data included in this report.-Continued

[NAWQA, National Water-Quality Assessment; ID, identification; no., number; LS, land surface; ft bls, foot below land surface; lat., latitude, in degrees and minutes; long., longitude in degrees and minutes; PAS, principal aquifer study; FL, Florida; na, not available; NGVD 29, National Geodetic Vertical Datum of 1929; NAVD 88, North American Vertical Datum of 1988; ETN, enhanced trends network; TX,

Texas; NM, New Mexico; LUS, land-use study; SC, South Carolina; FPS, flow-path study; MS, Mississippi; VFPS; vertical flow-path study; TN, Tennessee; OK, Oklahoma; AR, Arkansas; CA, California; MO, Missouri; KS, Kansas; MD, Maryland; MSS, modeling support study; DE, Delaware; DC, District of Columbia; MAS, major aquifer study; NV, Nevada; NY, New York; OH, Ohio; MI, Michigan; ID, Idaho;

NH, New Hampshire; WI, Wisconsin; OR, Oregon; MN, Minnesota; WA, Washington]

\begin{tabular}{|c|c|c|c|c|c|c|c|c|c|c|c|c|}
\hline \multirow{2}{*}{$\begin{array}{l}\text { Principal and } \\
\text { regional and (or) } \\
\text { other aquifer } \\
\text { information }\end{array}$} & \multirow{2}{*}{$\begin{array}{l}\text { Network } \\
\text { type }\end{array}$} & \multirow{2}{*}{$\begin{array}{l}\text { Network } \\
\text { name }\end{array}$} & \multirow{2}{*}{$\begin{array}{l}\text { NAWQA well } \\
\text { ID no. }\end{array}$} & \multirow{2}{*}{ State } & \multirow{2}{*}{ County } & \multirow{2}{*}{ Sample date } & \multirow{2}{*}{$\begin{array}{c}\text { Altitude } \\
\text { LS }\end{array}$} & \multirow{2}{*}{$\begin{array}{l}\text { Altitude } \\
\text { datum }\end{array}$} & \multirow{2}{*}{ Water use } & \multirow{2}{*}{$\begin{array}{c}\text { Well } \\
\text { depth } \\
\text { (ft bls) }\end{array}$} & \multicolumn{2}{|c|}{$\begin{array}{c}\text { Depth to perforation } \\
\text { (ft bls) }\end{array}$} \\
\hline & & & & & & & & & & & Top & Bottom \\
\hline $\begin{array}{l}\text { Columbia } \\
\text { Plateau } \\
\text { basaltic-rock } \\
\text { aquifers }\end{array}$ & PAS & clptpas 1 & CLPTPAS1-11 & WA & Yakima & $7 / 14 / 2016$ & 745 & NGVD 29 & Public supply & 385 & 305 & 375 \\
\hline $\begin{array}{l}\text { Columbia } \\
\text { Plateau } \\
\text { basaltic-rock } \\
\text { aquifers }\end{array}$ & PAS & clptpas 1 & CLPTPAS1-12 & WA & Grant & $7 / 18 / 2016$ & 1,317 & NAVD 88 & Public supply & 406 & 195 & 406 \\
\hline $\begin{array}{l}\text { Columbia } \\
\text { Plateau } \\
\text { basaltic-rock } \\
\text { aquifers }\end{array}$ & PAS & clptpas1 & CLPTPAS1-13 & WA & Douglas & $7 / 19 / 2016$ & 2,760 & NAVD 88 & Public supply & 605 & na & $605^{\mathrm{a}}$ \\
\hline $\begin{array}{l}\text { Columbia } \\
\text { Plateau } \\
\text { basaltic-rock } \\
\text { aquifers }\end{array}$ & PAS & clptpas 1 & CLPTPAS1-14 & WA & Lincoln & $7 / 20 / 2016$ & 1,692 & NGVD 29 & Public supply & 595 & 250 & 595 \\
\hline $\begin{array}{l}\text { Columbia } \\
\text { Plateau } \\
\text { basaltic-rock } \\
\text { aquifers }\end{array}$ & PAS & clptpas 1 & CLPTPAS1-15 & WA & Grant & $7 / 21 / 2016$ & 1,122 & NAVD 88 & Public supply & 466 & na & $466^{\mathrm{a}}$ \\
\hline $\begin{array}{l}\text { Columbia } \\
\text { Plateau } \\
\text { basaltic-rock } \\
\text { aquifers }\end{array}$ & PAS & clptpas1 & CLPTPAS1-16 & WA & Grant & $7 / 21 / 2016$ & 1,175 & NAVD 88 & Public supply & 743 & 669 & 740 \\
\hline $\begin{array}{l}\text { Columbia } \\
\text { Plateau } \\
\text { basaltic-rock } \\
\text { aquifers }\end{array}$ & PAS & clptpas1 & CLPTPAS1-17 & WA & Lincoln & $7 / 25 / 2016$ & 1,922 & NGVD 29 & Public supply & 149 & 60 & 208 \\
\hline $\begin{array}{l}\text { Columbia } \\
\text { Plateau } \\
\text { basaltic-rock } \\
\text { aquifers }\end{array}$ & PAS & clptpas 1 & CLPTPAS1-18 & WA & Lincoln & $7 / 25 / 2016$ & 2,480 & NGVD 29 & Public supply & 455 & na & $455^{a}$ \\
\hline
\end{tabular}


Table 1. Information about wells that have environmental data included in this report.-Continued

[NAWQA, National Water-Quality Assessment; ID, identification; no., number; LS, land surface; ft bls, foot below land surface; lat., latitude, in degrees and minutes; long., longitude in degrees and minutes; PAS, principal aquifer study; FL, Florida; na, not available; NGVD 29, National Geodetic Vertical Datum of 1929; NAVD 88, North American Vertical Datum of 1988; ETN, enhanced trends network; TX, Texas; NM, New Mexico; LUS, land-use study; SC, South Carolina; FPS, flow-path study; MS, Mississippi; VFPS; vertical flow-path study; TN, Tennessee; OK, Oklahoma; AR, Arkansas; CA, California; MO, Missouri; KS, Kansas; MD, Maryland; MSS, modeling support study; DE, Delaware; DC, District of Columbia; MAS, major aquifer study; NV, Nevada; NY, New York; OH, Ohio; MI, Michigan; ID, Idaho; NH, New Hampshire; WI, Wisconsin; OR, Oregon; MN, Minnesota; WA, Washington]

\begin{tabular}{|c|c|c|c|c|c|c|c|c|c|c|c|c|}
\hline \multirow{2}{*}{$\begin{array}{l}\text { Principal and } \\
\text { regional and (or) } \\
\text { other aquifer } \\
\text { information }\end{array}$} & \multirow{2}{*}{$\begin{array}{l}\text { Network } \\
\text { type }\end{array}$} & \multirow{2}{*}{$\begin{array}{l}\text { Network } \\
\text { name }\end{array}$} & \multirow{2}{*}{$\begin{array}{l}\text { NAWOA well } \\
\text { ID no. }\end{array}$} & \multirow{2}{*}{ State } & \multirow{2}{*}{ County } & \multirow{2}{*}{ Sample date } & \multirow{2}{*}{$\begin{array}{l}\text { Altitude } \\
\text { LS }\end{array}$} & \multirow{2}{*}{$\begin{array}{l}\text { Altitude } \\
\text { datum }\end{array}$} & \multirow{2}{*}{ Water use } & \multirow{2}{*}{$\begin{array}{c}\text { Well } \\
\text { depth } \\
\text { (ft bls) }\end{array}$} & \multicolumn{2}{|c|}{$\begin{array}{c}\text { Depth to perforation } \\
\text { (ft bls) }\end{array}$} \\
\hline & & & & & & & & & & & Top & Bottom \\
\hline $\begin{array}{l}\text { Columbia } \\
\text { Plateau } \\
\text { basaltic-rock } \\
\text { aquifers }\end{array}$ & PAS & clptpas 1 & CLPTPAS1-19 & WA & Lincoln & $7 / 26 / 2016$ & 2,141 & NGVD 29 & Public supply & 300 & 180 & 300 \\
\hline $\begin{array}{l}\text { Columbia } \\
\text { Plateau } \\
\text { basaltic-rock } \\
\text { aquifers }\end{array}$ & PAS & clptpas1 & CLPTPAS1-20 & WA & Spokane & $7 / 26 / 2016$ & 2,444 & NAVD 88 & Public supply & 260 & 187 & 260 \\
\hline $\begin{array}{l}\text { Columbia } \\
\text { Plateau } \\
\text { basaltic-rock } \\
\text { aquifers }\end{array}$ & PAS & clptpas1 & CLPTPAS1-21 & WA & Spokane & $7 / 27 / 2016$ & 2,364 & NAVD 88 & Public supply & 710 & 530 & 710 \\
\hline $\begin{array}{l}\text { Columbia } \\
\text { Plateau } \\
\text { basaltic-rock } \\
\text { aquifers }\end{array}$ & PAS & clptpas1 & CLPTPAS1-22 & WA & Grant & $7 / 28 / 2016$ & 1,210 & NAVD 88 & Public supply & 1,240 & 686 & 1,240 \\
\hline $\begin{array}{l}\text { Columbia } \\
\text { Plateau } \\
\text { basaltic-rock } \\
\text { aquifers }\end{array}$ & PAS & clptpas1 & CLPTPAS1-23 & WA & Adams & $8 / 1 / 2016$ & 1,979 & NGVD 29 & Public supply & 789 & 34 & 34 \\
\hline $\begin{array}{l}\text { Columbia } \\
\text { Plateau } \\
\text { basaltic-rock } \\
\text { aquifers }\end{array}$ & PAS & clptpas1 & CLPTPAS1-24 & WA & Whitman & $8 / 1 / 2016$ & 1,984 & NAVD 88 & Public supply & 285 & 215 & 285 \\
\hline $\begin{array}{l}\text { Columbia } \\
\text { Plateau } \\
\text { basaltic-rock } \\
\text { aquifers }\end{array}$ & PAS & clptpas1 & CLPTPAS1-25 & WA & Whitman & $8 / 2 / 2016$ & 2,578 & NAVD 88 & Public supply & 315 & 211 & 315 \\
\hline $\begin{array}{l}\text { Columbia } \\
\text { Plateau } \\
\text { basaltic-rock } \\
\text { aquifers }\end{array}$ & PAS & clptpas1 & CLPTPAS1-26 & WA & Garfield & $8 / 3 / 2016$ & 1,891 & NAVD 88 & Public supply & 347 & 60 & 347 \\
\hline
\end{tabular}


Table 1. Information about wells that have environmental data included in this report.-Continued

[NAWQA, National Water-Quality Assessment; ID, identification; no., number; LS, land surface; ft bls, foot below land surface; lat., latitude, in degrees and minutes; long., longitude in degrees and minutes; PAS, principal aquifer study; FL, Florida; na, not available; NGVD 29, National Geodetic Vertical Datum of 1929; NAVD 88, North American Vertical Datum of 1988; ETN, enhanced trends network; TX,

Texas; NM, New Mexico; LUS, land-use study; SC, South Carolina; FPS, flow-path study; MS, Mississippi; VFPS; vertical flow-path study; TN, Tennessee; OK, Oklahoma; AR, Arkansas; CA, California; MO, Missouri; KS, Kansas; MD, Maryland; MSS, modeling support study; DE, Delaware; DC, District of Columbia; MAS, major aquifer study; NV, Nevada; NY, New York; OH, Ohio; MI, Michigan; ID, Idaho;

NH, New Hampshire; WI, Wisconsin; OR, Oregon; MN, Minnesota; WA, Washington]

\begin{tabular}{|c|c|c|c|c|c|c|c|c|c|c|c|c|}
\hline \multirow{2}{*}{$\begin{array}{l}\text { Principal and } \\
\text { regional and (or) } \\
\text { other aquifer } \\
\text { information }\end{array}$} & \multirow{2}{*}{$\begin{array}{l}\text { Network } \\
\text { type }\end{array}$} & \multirow{2}{*}{$\begin{array}{l}\text { Network } \\
\text { name }\end{array}$} & \multirow{2}{*}{$\begin{array}{l}\text { NAWQA well } \\
\text { ID no. }\end{array}$} & \multirow{2}{*}{ State } & \multirow{2}{*}{ County } & \multirow{2}{*}{ Sample date } & \multirow{2}{*}{$\begin{array}{l}\text { Altitude } \\
\text { LS }\end{array}$} & \multirow{2}{*}{$\begin{array}{l}\text { Altitude } \\
\text { datum }\end{array}$} & \multirow{2}{*}{ Water use } & \multirow{2}{*}{$\begin{array}{c}\text { Well } \\
\text { depth } \\
\text { (ft bls) }\end{array}$} & \multicolumn{2}{|c|}{$\begin{array}{c}\text { Depth to perforation } \\
\text { (ft bls) }\end{array}$} \\
\hline & & & & & & & & & & & Top & Bottom \\
\hline $\begin{array}{l}\text { Columbia } \\
\text { Plateau } \\
\text { basaltic-rock } \\
\text { aquifers }\end{array}$ & PAS & clptpas1 & CLPTPAS1-27 & WA & Garfield & $8 / 3 / 2016$ & 1,892 & NAVD 88 & Public supply & 997 & 373 & 997 \\
\hline $\begin{array}{l}\text { Columbia } \\
\text { Plateau } \\
\text { basaltic-rock } \\
\text { aquifers }\end{array}$ & PAS & clptpas 1 & CLPTPAS1-28 & WA & Walla Walla & $8 / 8 / 2016$ & 1,624 & NAVD 88 & Public supply & 512 & 42 & 512 \\
\hline $\begin{array}{l}\text { Columbia } \\
\text { Plateau } \\
\text { basaltic-rock } \\
\text { aquifers }\end{array}$ & PAS & clptpas1 & CLPTPAS1-29 & WA & Walla Walla & $8 / 8 / 2016$ & 1,062 & NAVD 88 & Public supply & 484 & 145 & 484 \\
\hline $\begin{array}{l}\text { Columbia } \\
\text { Plateau } \\
\text { basaltic-rock } \\
\text { aquifers }\end{array}$ & PAS & clptpas 1 & CLPTPAS1-30 & WA & Walla Walla & $8 / 9 / 2016$ & 802 & NAVD 88 & Public supply & 708 & 554 & 708 \\
\hline $\begin{array}{l}\text { Columbia } \\
\text { Plateau } \\
\text { basaltic-rock } \\
\text { aquifers }\end{array}$ & PAS & clptpas1 & CLPTPAS1-31 & WA & Walla Walla & 8/9/2016 & 802 & NAVD 88 & Public supply & 787 & 585 & 787 \\
\hline $\begin{array}{l}\text { Columbia } \\
\text { Plateau } \\
\text { basaltic-rock } \\
\text { aquifers }\end{array}$ & PAS & clptpas 1 & CLPTPAS1-32 & WA & Franklin & $8 / 10 / 2016$ & 723 & NAVD 88 & Public supply & 363 & 352 & 363 \\
\hline $\begin{array}{l}\text { Columbia } \\
\text { Plateau } \\
\text { basaltic-rock } \\
\text { aquifers }\end{array}$ & PAS & clptpas1 & CLPTPAS1-33 & WA & Klickitat & $8 / 16 / 2016$ & 2,168 & NAVD 88 & Public supply & 240 & 38 & 240 \\
\hline $\begin{array}{l}\text { Columbia } \\
\text { Plateau } \\
\text { basaltic-rock } \\
\text { aquifers }\end{array}$ & PAS & clptpas1 & CLPTPAS1-34 & WA & Klickitat & $8 / 17 / 2016$ & 1,381 & NAVD 88 & Public supply & 600 & 50 & 600 \\
\hline
\end{tabular}


Table 1. Information about wells that have environmental data included in this report.-Continued

[NAWQA, National Water-Quality Assessment; ID, identification; no., number; LS, land surface; ft bls, foot below land surface; lat., latitude, in degrees and minutes; long., longitude in degrees and minutes; PAS, principal aquifer study; FL, Florida; na, not available; NGVD 29, National Geodetic Vertical Datum of 1929; NAVD 88, North American Vertical Datum of 1988; ETN, enhanced trends network; TX, Texas; NM, New Mexico; LUS, land-use study; SC, South Carolina; FPS, flow-path study; MS, Mississippi; VFPS; vertical flow-path study; TN, Tennessee; OK, Oklahoma; AR, Arkansas; CA, California; MO, Missouri; KS, Kansas; MD, Maryland; MSS, modeling support study; DE, Delaware; DC, District of Columbia; MAS, major aquifer study; NV, Nevada; NY, New York; OH, Ohio; MI, Michigan; ID, Idaho; NH, New Hampshire; WI, Wisconsin; OR, Oregon; MN, Minnesota; WA, Washington]

\begin{tabular}{|c|c|c|c|c|c|c|c|c|c|c|c|c|}
\hline \multirow{2}{*}{$\begin{array}{l}\text { Principal and } \\
\text { regional and (or) } \\
\text { other aquifer } \\
\text { information }\end{array}$} & \multirow{2}{*}{$\begin{array}{l}\text { Network } \\
\text { type }\end{array}$} & \multirow{2}{*}{$\begin{array}{l}\text { Network } \\
\text { name }\end{array}$} & \multirow{2}{*}{$\begin{array}{l}\text { NAWQA well } \\
\text { ID no. }\end{array}$} & \multirow{2}{*}{ State } & \multirow{2}{*}{ County } & \multirow{2}{*}{ Sample date } & \multirow{2}{*}{$\begin{array}{l}\text { Altitude } \\
\text { LS }\end{array}$} & \multirow{2}{*}{$\begin{array}{l}\text { Altitude } \\
\text { datum }\end{array}$} & \multirow{2}{*}{ Water use } & \multirow{2}{*}{$\begin{array}{c}\text { Well } \\
\text { depth } \\
\text { (ft bls) }\end{array}$} & \multicolumn{2}{|c|}{$\begin{array}{c}\text { Depth to perforation } \\
\text { (ft bls) }\end{array}$} \\
\hline & & & & & & & & & & & Top & Bottom \\
\hline \multirow{2}{*}{$\begin{array}{l}\text { Columbia } \\
\text { Plateau } \\
\text { basin-fill and } \\
\text { basaltic-rock } \\
\text { aquifers }\end{array}$} & PAS/ & clptpas1/ & CLPTPAS1-35/ & \multirow[t]{2}{*}{ OR } & \multirow[t]{2}{*}{ Umatilla } & \multirow{2}{*}{$\begin{array}{c}1 / 21,3 / 18 \\
4 / 20,6 / 23 \\
7 / 27,9 / 9 \\
11 / 18 / 2016\end{array}$} & \multirow[t]{2}{*}{693} & \multirow[t]{2}{*}{ NAVD 88} & \multirow[t]{2}{*}{ Public supply } & \multirow[t]{2}{*}{1,116} & \multirow[t]{2}{*}{926} & \multirow[t]{2}{*}{1,100} \\
\hline & ETN & clptetn1 & CLPTETN1-04 & & & & & & & & & \\
\hline $\begin{array}{l}\text { Columbia } \\
\text { Plateau } \\
\text { basaltic-rock } \\
\text { aquifers }\end{array}$ & PAS & clptpas1 & CLPTPAS1-36 & OR & Wasco & $7 / 11 / 2016$ & 168 & NAVD 88 & Public supply & 242 & 165 & 242 \\
\hline $\begin{array}{l}\text { Columbia } \\
\text { Plateau } \\
\text { basaltic-rock } \\
\text { aquifers }\end{array}$ & PAS & clptpas1 & CLPTPAS1-37 & OR & Union & $7 / 12 / 2016$ & 2,798 & NAVD 88 & Public supply & 2,434 & $1,132.80$ & $2,429.50$ \\
\hline $\begin{array}{l}\text { Columbia } \\
\text { Plateau } \\
\text { basaltic-rock } \\
\text { aquifers }\end{array}$ & PAS & clptpas1 & CLPTPAS1-38 & OR & Wallowa & 7/13/2016 & 3,832 & NAVD 88 & Public supply & 1315 & 440 & 1,315 \\
\hline $\begin{array}{l}\text { Columbia } \\
\text { Plateau } \\
\text { basaltic-rock } \\
\text { aquifers }\end{array}$ & PAS & clptpas1 & CLPTPAS1-39 & OR & Wallowa & $7 / 14 / 2016$ & 2,947 & NAVD 88 & Public supply & 870 & 840 & $870^{b}$ \\
\hline $\begin{array}{l}\text { Columbia } \\
\text { Plateau } \\
\text { basaltic-rock } \\
\text { aquifers }\end{array}$ & PAS & clptpas1 & CLPTPAS1-40 & OR & Gilliam & $7 / 25 / 2016$ & 463 & NAVD 88 & Public supply & 470 & 195 & 470 \\
\hline $\begin{array}{l}\text { Columbia } \\
\text { Plateau } \\
\text { basaltic-rock } \\
\text { aquifers }\end{array}$ & PAS & clptpas 1 & CLPTPAS1-41 & OR & Union & $7 / 26 / 2016$ & 2,730 & NGVD 29 & Public supply & 600 & 482 & 596 \\
\hline $\begin{array}{l}\text { Columbia } \\
\text { Plateau } \\
\text { basaltic-rock } \\
\text { aquifers }\end{array}$ & PAS & clptpas1 & CLPTPAS1-42 & OR & Umatilla & $8 / 1 / 2016$ & 485 & NGVD 29 & Public supply & 785 & 535 & 785 \\
\hline
\end{tabular}


Table 1. Information about wells that have environmental data included in this report.-Continued

[NAWQA, National Water-Quality Assessment; ID, identification; no., number; LS, land surface; ft bls, foot below land surface; lat., latitude, in degrees and minutes; long., longitude in degrees and minutes; PAS, principal aquifer study; FL, Florida; na, not available; NGVD 29, National Geodetic Vertical Datum of 1929; NAVD 88, North American Vertical Datum of 1988; ETN, enhanced trends network; TX,

Texas; NM, New Mexico; LUS, land-use study; SC, South Carolina; FPS, flow-path study; MS, Mississippi; VFPS; vertical flow-path study; TN, Tennessee; OK, Oklahoma; AR, Arkansas; CA, California; MO, Missouri; KS, Kansas; MD, Maryland; MSS, modeling support study; DE, Delaware; DC, District of Columbia; MAS, major aquifer study; NV, Nevada; NY, New York; OH, Ohio; MI, Michigan; ID, Idaho;

NH, New Hampshire; WI, Wisconsin; OR, Oregon; MN, Minnesota; WA, Washington]

\begin{tabular}{|c|c|c|c|c|c|c|c|c|c|c|c|c|}
\hline \multirow{2}{*}{$\begin{array}{l}\text { Principal and } \\
\text { regional and (or) } \\
\text { other aquifer } \\
\text { information }\end{array}$} & \multirow{2}{*}{$\begin{array}{c}\text { Network } \\
\text { type }\end{array}$} & \multirow{2}{*}{$\begin{array}{l}\text { Network } \\
\text { name }\end{array}$} & \multirow{2}{*}{$\begin{array}{l}\text { NAWQA well } \\
\text { ID no. }\end{array}$} & \multirow{2}{*}{ State } & \multirow{2}{*}{ County } & \multirow{2}{*}{ Sample date } & \multirow{2}{*}{$\begin{array}{c}\text { Altitude } \\
\text { LS }\end{array}$} & \multirow{2}{*}{$\begin{array}{l}\text { Altitude } \\
\text { datum }\end{array}$} & \multirow{2}{*}{ Water use } & \multirow{2}{*}{$\begin{array}{c}\text { Well } \\
\text { depth } \\
\text { (ft bls) }\end{array}$} & \multicolumn{2}{|c|}{$\begin{array}{c}\text { Depth to perforation } \\
\text { (ft bls) }\end{array}$} \\
\hline & & & & & & & & & & & Top & Bottom \\
\hline $\begin{array}{l}\text { Columbia } \\
\text { Plateau } \\
\text { basaltic-rock } \\
\text { aquifers }\end{array}$ & PAS & clptpas1 & CLPTPAS1-43 & OR & Union & $8 / 2 / 2016$ & 2,792 & NGVD 29 & Public supply & 1,200 & 495.5 & 1,200 \\
\hline $\begin{array}{l}\text { Columbia } \\
\text { Plateau } \\
\text { basaltic-rock } \\
\text { aquifers }\end{array}$ & PAS & clptpas1 & CLPTPAS1-44 & OR & Morrow & $8 / 3 / 2016$ & 2,700 & NGVD 29 & Public supply & 422 & 34 & 422 \\
\hline $\begin{array}{l}\text { Columbia } \\
\text { Plateau } \\
\text { basaltic-rock } \\
\text { aquifers }\end{array}$ & PAS & clptpas1 & CLPTPAS1-45 & OR & Wasco & $8 / 4 / 2016$ & 3,070 & NAVD 88 & Public supply & 150 & 61 & 150 \\
\hline $\begin{array}{l}\text { Columbia } \\
\text { Plateau } \\
\text { basaltic-rock } \\
\text { aquifers }\end{array}$ & PAS & clptpas 1 & CLPTPAS1-46 & OR & Umatilla & 8/8/2016 & 1,649 & NAVD 88 & Public supply & 309 & 31 & 309 \\
\hline $\begin{array}{l}\text { Columbia } \\
\text { Plateau } \\
\text { basaltic-rock } \\
\text { aquifers }\end{array}$ & PAS & clptpas 1 & CLPTPAS1-47 & OR & Umatilla & $8 / 9 / 2016$ & 3,403 & NGVD 29 & Public supply & 580 & 501 & 580 \\
\hline $\begin{array}{l}\text { Columbia } \\
\text { Plateau } \\
\text { basaltic-rock } \\
\text { aquifers }\end{array}$ & PAS & clptpas1 & CLPTPAS1-48 & OR & Umatilla & $8 / 10 / 2016$ & 1,005 & NAVD 88 & Public supply & 502 & 212 & 502 \\
\hline $\begin{array}{l}\text { Columbia } \\
\text { Plateau } \\
\text { basaltic-rock } \\
\text { aquifers }\end{array}$ & PAS & clptpas1 & CLPTPAS1-49 & OR & Gilliam & $8 / 11 / 2016$ & 2,300 & NGVD 29 & Public supply & 174 & 55 & 174 \\
\hline $\begin{array}{l}\text { Columbia } \\
\text { Plateau } \\
\text { basaltic-rock } \\
\text { aquifers }\end{array}$ & PAS & clptpas 1 & CLPTPAS1-50 & OR & Wasco & $8 / 22 / 2016$ & 13,40 & NGVD 29 & Public supply & 624 & 385 & 624 \\
\hline
\end{tabular}


Table 1. Information about wells that have environmental data included in this report.-Continued

[NAWQA, National Water-Quality Assessment; ID, identification; no., number; LS, land surface; ft bls, foot below land surface; lat., latitude, in degrees and minutes; long., longitude in degrees and minutes; PAS, principal aquifer study; FL, Florida; na, not available; NGVD 29, National Geodetic Vertical Datum of 1929; NAVD 88, North American Vertical Datum of 1988; ETN, enhanced trends network; TX, Texas; NM, New Mexico; LUS, land-use study; SC, South Carolina; FPS, flow-path study; MS, Mississippi; VFPS; vertical flow-path study; TN, Tennessee; OK, Oklahoma; AR, Arkansas; CA, California; MO, Missouri; KS, Kansas; MD, Maryland; MSS, modeling support study; DE, Delaware; DC, District of Columbia; MAS, major aquifer study; NV, Nevada; NY, New York; OH, Ohio; MI, Michigan; ID, Idaho; NH, New Hampshire; WI, Wisconsin; OR, Oregon; MN, Minnesota; WA, Washington]

\begin{tabular}{|c|c|c|c|c|c|c|c|c|c|c|c|c|}
\hline \multirow{2}{*}{$\begin{array}{l}\text { Principal and } \\
\text { regional and (or) } \\
\text { other aquifer } \\
\text { information }\end{array}$} & \multirow{2}{*}{$\begin{array}{l}\text { Network } \\
\text { type }\end{array}$} & \multirow{2}{*}{$\begin{array}{l}\text { Network } \\
\text { name }\end{array}$} & \multirow{2}{*}{$\begin{array}{l}\text { NAWQA well } \\
\text { ID no. }\end{array}$} & \multirow{2}{*}{ State } & \multirow{2}{*}{ County } & \multirow{2}{*}{ Sample date } & \multirow{2}{*}{$\begin{array}{l}\text { Altitude } \\
\text { LS }\end{array}$} & \multirow{2}{*}{$\begin{array}{l}\text { Altitude } \\
\text { datum }\end{array}$} & \multirow{2}{*}{ Water use } & \multirow{2}{*}{$\begin{array}{c}\text { Well } \\
\text { depth } \\
\text { (ft bls) }\end{array}$} & \multicolumn{2}{|c|}{$\begin{array}{c}\text { Depth to perforation } \\
\text { (ft bls) }\end{array}$} \\
\hline & & & & & & & & & & & Top & Bottom \\
\hline $\begin{array}{l}\text { Columbia } \\
\text { Plateau } \\
\text { basaltic-rock } \\
\text { aquifers }\end{array}$ & PAS & clptpas1 & CLPTPAS1-51 & OR & Morrow & $8 / 23 / 2016$ & 1,100 & NGVD 29 & Public supply & 675 & 25 & 675 \\
\hline $\begin{array}{l}\text { Columbia } \\
\text { Plateau } \\
\text { basaltic-rock } \\
\text { aquifers }\end{array}$ & PAS & clptpas1 & CLPTPAS1-52 & OR & Grant & $8 / 24 / 2016$ & 3,710 & NGVD 29 & Public supply & 272 & 198 & 272 \\
\hline $\begin{array}{l}\text { Columbia } \\
\text { Plateau } \\
\text { basaltic-rock } \\
\text { aquifers }\end{array}$ & PAS & clptpas1 & CLPTPAS1-53 & OR & Wasco & $8 / 25 / 2016$ & 1,765 & NAVD 88 & Public supply & 340 & 133 & 340 \\
\hline $\begin{array}{l}\text { Columbia } \\
\text { Plateau } \\
\text { basaltic-rock } \\
\text { aquifers }\end{array}$ & PAS & clptpas1 & CLPTPAS1-54 & OR & Sherman & $9 / 1 / 2016$ & 2,292 & NAVD 88 & Public supply & 450 & 284 & 450 \\
\hline $\begin{array}{l}\text { Columbia } \\
\text { Plateau } \\
\text { basaltic-rock } \\
\text { aquifers }\end{array}$ & PAS & clptpas1 & CLPTPAS1-55 & WA & Asotin & $8 / 4 / 2016$ & 1,180 & NGVD 29 & Public supply & 1,340 & 653 & 1,340 \\
\hline $\begin{array}{l}\text { Columbia } \\
\text { Plateau } \\
\text { basaltic-rock } \\
\text { aquifers }\end{array}$ & PAS & clptpas1 & CLPTPAS1-56 & ID & Idaho & $8 / 1 / 2016$ & 1,440 & NGVD 29 & Public supply & 195 & 28 & 195 \\
\hline $\begin{array}{l}\text { Columbia } \\
\text { Plateau } \\
\text { basaltic-rock } \\
\text { aquifers }\end{array}$ & PAS & clptpas1 & CLPTPAS1-57 & ID & Idaho & $8 / 2 / 2016$ & 3,255 & NAVD 88 & Public supply & 101 & na & $101^{\mathrm{a}}$ \\
\hline $\begin{array}{l}\text { Columbia } \\
\text { Plateau } \\
\text { basaltic-rock } \\
\text { aquifers }\end{array}$ & PAS & clptpas1 & CLPTPAS1-58 & ID & Idaho & $8 / 3 / 2016$ & 3,752 & NAVD 88 & Public supply & na & na & na \\
\hline
\end{tabular}


Table 1. Information about wells that have environmental data included in this report.-Continued

[NAWQA, National Water-Quality Assessment; ID, identification; no., number; LS, land surface; ft bls, foot below land surface; lat., latitude, in degrees and minutes; long., longitude in degrees and minutes; PAS, principal aquifer study; FL, Florida; na, not available; NGVD 29, National Geodetic Vertical Datum of 1929; NAVD 88, North American Vertical Datum of 1988; ETN, enhanced trends network; TX,

Texas; NM, New Mexico; LUS, land-use study; SC, South Carolina; FPS, flow-path study; MS, Mississippi; VFPS; vertical flow-path study; TN, Tennessee; OK, Oklahoma; AR, Arkansas; CA, California; MO, Missouri; KS, Kansas; MD, Maryland; MSS, modeling support study; DE, Delaware; DC, District of Columbia; MAS, major aquifer study; NV, Nevada; NY, New York; OH, Ohio; MI, Michigan; ID, Idaho;

NH, New Hampshire; WI, Wisconsin; OR, Oregon; MN, Minnesota; WA, Washington]

\begin{tabular}{|c|c|c|c|c|c|c|c|c|c|c|c|c|}
\hline \multirow{2}{*}{$\begin{array}{l}\text { Principal and } \\
\text { regional and (or) } \\
\text { other aquifer } \\
\text { information }\end{array}$} & \multirow{2}{*}{$\begin{array}{l}\text { Network } \\
\text { type }\end{array}$} & \multirow{2}{*}{$\begin{array}{c}\text { Network } \\
\text { name }\end{array}$} & \multirow{2}{*}{$\begin{array}{l}\text { NAWQA well } \\
\text { ID no. }\end{array}$} & \multirow{2}{*}{ State } & \multirow{2}{*}{ County } & \multirow{2}{*}{ Sample date } & \multirow{2}{*}{$\begin{array}{c}\text { Altitude } \\
\text { LS }\end{array}$} & \multirow{2}{*}{$\begin{array}{l}\text { Altitude } \\
\text { datum }\end{array}$} & \multirow{2}{*}{ Water use } & \multirow{2}{*}{$\begin{array}{c}\text { Well } \\
\text { depth } \\
\text { (ft bls) }\end{array}$} & \multicolumn{2}{|c|}{$\begin{array}{c}\text { Depth to perforation } \\
\text { (ft bls) }\end{array}$} \\
\hline & & & & & & & & & & & Top & Bottom \\
\hline $\begin{array}{l}\text { Columbia } \\
\text { Plateau } \\
\text { basaltic-rock } \\
\text { aquifers }\end{array}$ & PAS & clptpas1 & CLPTPAS1-59 & ID & Lewis & $8 / 3 / 2016$ & 3,917 & NAVD 88 & Public supply & 382 & na & $382^{a}$ \\
\hline $\begin{array}{l}\text { Columbia } \\
\text { Plateau } \\
\text { basaltic-rock } \\
\text { aquifers }\end{array}$ & PAS & clptpas 1 & CLPTPAS1-60 & ID & Latah & $8 / 4 / 2016$ & 2,617 & NGVD 29 & Public supply & 1,458 & 1,047 & 1,458 \\
\hline $\begin{array}{l}\text { Columbia } \\
\text { Plateau } \\
\text { basin-fill and } \\
\text { basaltic-rock } \\
\text { aquifers }\end{array}$ & VFPS & clptvfps 1 & CLPTVFPS1-02 & WA & Grant & $7 / 30 / 2014$ & 1,246 & NAVD 88 & Observation & 21.5 & 18.5 & 21.5 \\
\hline $\begin{array}{l}\text { Columbia } \\
\text { Plateau } \\
\text { basin-fill and } \\
\text { basaltic-rock } \\
\text { aquifers }\end{array}$ & VFPS & clptvfps 1 & CLPTVFPS1-03 & WA & Franklin & $8 / 18 / 2014$ & 457 & NAVD 88 & Public supply & 155 & na & $155^{\mathrm{a}}$ \\
\hline $\begin{array}{l}\text { Columbia } \\
\text { Plateau } \\
\text { basin-fill and } \\
\text { basaltic-rock } \\
\text { aquifers }\end{array}$ & VFPS & clptvfps 1 & CLPTVFPS1-06 & WA & Franklin & $8 / 26 / 2014$ & 423 & NAVD 88 & Observation & 88 & 83 & 88 \\
\hline $\begin{array}{l}\text { Columbia } \\
\text { Plateau } \\
\text { basin-fill and } \\
\text { basaltic-rock } \\
\text { aquifers }\end{array}$ & VFPS & clptvfps1 & CLPTVFPS1-08 & WA & Grant & $9 / 19 / 2014$ & 1,236 & NAVD 88 & Observation & 112 & 107 & 112 \\
\hline $\begin{array}{l}\text { Columbia } \\
\text { Plateau } \\
\text { basin-fill and } \\
\text { basaltic-rock } \\
\text { aquifers }\end{array}$ & VFPS & clptvfps 1 & CLPTVFPS1-09 & WA & Grant & $7 / 24 / 2014$ & 1,202 & NAVD 88 & Public supply & 170 & 19 & 170 \\
\hline
\end{tabular}


Table 1. Information about wells that have environmental data included in this report.-Continued

[NAWQA, National Water-Quality Assessment; ID, identification; no., number; LS, land surface; $\mathrm{ft}$ bls, foot below land surface; lat., latitude, in degrees and minutes; long., longitude in degrees and minutes; PAS, principal aquifer study; FL, Florida; na, not available; NGVD 29, National Geodetic Vertical Datum of 1929; NAVD 88, North American Vertical Datum of 1988; ETN, enhanced trends network; TX, Texas; NM, New Mexico; LUS, land-use study; SC, South Carolina; FPS, flow-path study; MS, Mississippi; VFPS; vertical flow-path study; TN, Tennessee; OK, Oklahoma; AR, Arkansas; CA, California; MO, Missouri; KS, Kansas; MD, Maryland; MSS, modeling support study; DE, Delaware; DC, District of Columbia; MAS, major aquifer study; NV, Nevada; NY, New York; OH, Ohio; MI, Michigan; ID, Idaho; NH, New Hampshire; WI, Wisconsin; OR, Oregon; MN, Minnesota; WA, Washington]

\begin{tabular}{|c|c|c|c|c|c|c|c|c|c|c|c|c|}
\hline \multirow{2}{*}{$\begin{array}{l}\text { Principal and } \\
\text { regional and (or) } \\
\text { other aquifer } \\
\text { information }\end{array}$} & \multirow{2}{*}{$\begin{array}{l}\text { Network } \\
\text { type }\end{array}$} & \multirow{2}{*}{$\begin{array}{l}\text { Network } \\
\text { name }\end{array}$} & \multirow{2}{*}{$\begin{array}{l}\text { NAWQA well } \\
\text { ID no. }\end{array}$} & \multirow{2}{*}{ State } & \multirow{2}{*}{ County } & \multirow{2}{*}{ Sample date } & \multirow{2}{*}{$\begin{array}{l}\text { Altitude } \\
\text { LS }\end{array}$} & \multirow{2}{*}{$\begin{array}{c}\text { Altitude } \\
\text { datum }\end{array}$} & \multirow{2}{*}{ Water use } & \multirow{2}{*}{$\begin{array}{r}\text { Well } \\
\text { depth } \\
\text { (ft bls) }\end{array}$} & \multicolumn{2}{|c|}{$\begin{array}{c}\text { Depth to perforation } \\
\text { (ft bls) }\end{array}$} \\
\hline & & & & & & & & & & & Top & Bottom \\
\hline $\begin{array}{l}\text { Columbia } \\
\text { Plateau } \\
\text { basin-fill and } \\
\text { basaltic-rock } \\
\text { aquifers }\end{array}$ & VFPS & clptvfps1 & CLPTVFPS1-10 & WA & Grant & $7 / 24 / 2014$ & 1,117 & NAVD 88 & Observation & 38.5 & 33.5 & 38.5 \\
\hline $\begin{array}{l}\text { Columbia } \\
\text { Plateau } \\
\text { basin-fill and } \\
\text { basaltic-rock } \\
\text { aquifers }\end{array}$ & VFPS & clptvfps1 & CLPTVFPS1-11 & WA & Adams & $7 / 23 / 2014$ & $1,256.40$ & NAVD 88 & Public supply & 430 & 47 & 430 \\
\hline $\begin{array}{l}\text { Columbia } \\
\text { Plateau } \\
\text { basin-fill and } \\
\text { basaltic-rock } \\
\text { aquifers }\end{array}$ & VFPS & clptvfps 1 & CLPTVFPS1-12 & WA & Grant & $7 / 23 / 2014$ & 1,144 & NAVD 88 & Observation & 31 & 25 & 30 \\
\hline $\begin{array}{l}\text { Columbia } \\
\text { Plateau } \\
\text { basin-fill and } \\
\text { basaltic-rock } \\
\text { aquifers }\end{array}$ & VFPS & clptvfps 1 & CLPTVFPS1-13 & WA & Grant & $7 / 28 / 2014$ & 1,063 & NAVD 88 & Public supply & 180 & 73 & 180 \\
\hline $\begin{array}{l}\text { Columbia } \\
\text { Plateau } \\
\text { basin-fill and } \\
\text { basaltic-rock } \\
\text { aquifers }\end{array}$ & VFPS & clptvfps 1 & CLPTVFPS1-14 & WA & Grant & $7 / 30 / 2014$ & 1,132 & NAVD 88 & Observation & 29 & 24 & 29 \\
\hline $\begin{array}{l}\text { Columbia } \\
\text { Plateau } \\
\text { basin-fill and } \\
\text { basaltic-rock } \\
\text { aquifers }\end{array}$ & VFPS & clptvfps 1 & CLPTVFPS1-16 & WA & Adams & $8 / 12 / 2014$ & $1,205.10$ & NAVD 88 & Observation & 34 & 29 & 34 \\
\hline
\end{tabular}


Table 1. Information about wells that have environmental data included in this report.-Continued

[NAWQA, National Water-Quality Assessment; ID, identification; no., number; LS, land surface; ft bls, foot below land surface; lat., latitude, in degrees and minutes; long., longitude in degrees and minutes; PAS, principal aquifer study; FL, Florida; na, not available; NGVD 29, National Geodetic Vertical Datum of 1929; NAVD 88, North American Vertical Datum of 1988; ETN, enhanced trends network; TX,

Texas; NM, New Mexico; LUS, land-use study; SC, South Carolina; FPS, flow-path study; MS, Mississippi; VFPS; vertical flow-path study; TN, Tennessee; OK, Oklahoma; AR, Arkansas; CA, California; MO, Missouri; KS, Kansas; MD, Maryland; MSS, modeling support study; DE, Delaware; DC, District of Columbia; MAS, major aquifer study; NV, Nevada; NY, New York; OH, Ohio; MI, Michigan; ID, Idaho;

NH, New Hampshire; WI, Wisconsin; OR, Oregon; MN, Minnesota; WA, Washington]

\begin{tabular}{|c|c|c|c|c|c|c|c|c|c|c|c|c|}
\hline \multirow{2}{*}{$\begin{array}{l}\text { Principal and } \\
\text { regional and (or) } \\
\text { other aquifer } \\
\text { information }\end{array}$} & \multirow{2}{*}{$\begin{array}{c}\text { Network } \\
\text { type }\end{array}$} & \multirow{2}{*}{$\begin{array}{c}\text { Network } \\
\text { name }\end{array}$} & \multirow{2}{*}{$\begin{array}{l}\text { NAWOA well } \\
\text { ID no. }\end{array}$} & \multirow{2}{*}{ State } & \multirow{2}{*}{ County } & \multirow{2}{*}{ Sample date } & \multirow{2}{*}{$\begin{array}{l}\text { Altitude } \\
\text { LS }\end{array}$} & \multirow{2}{*}{$\begin{array}{c}\text { Altitude } \\
\text { datum }\end{array}$} & \multirow{2}{*}{ Water use } & \multirow{2}{*}{$\begin{array}{c}\text { Well } \\
\text { depth } \\
\text { (ft bls) }\end{array}$} & \multicolumn{2}{|c|}{$\begin{array}{c}\text { Depth to perforation } \\
\text { (ft bls) }\end{array}$} \\
\hline & & & & & & & & & & & Top & Bottom \\
\hline $\begin{array}{l}\text { Columbia } \\
\text { Plateau } \\
\text { basin-fill and } \\
\text { basaltic-rock } \\
\text { aquifers }\end{array}$ & VFPS & clptvfps1 & CLPTVFPS1-17 & WA & Adams & $9 / 11 / 2014$ & 1,044 & NAVD 88 & Public supply & 450 & 190 & 450 \\
\hline $\begin{array}{l}\text { Columbia } \\
\text { Plateau } \\
\text { basin-fill and } \\
\text { basaltic-rock } \\
\text { aquifers }\end{array}$ & VFPS & clptvfps1 & CLPTVFPS1-18 & WA & Adams & $8 / 12 / 2014$ & $1,078.80$ & NAVD 88 & Observation & 38 & 33 & 38 \\
\hline $\begin{array}{l}\text { Columbia } \\
\text { Plateau } \\
\text { basin-fill and } \\
\text { basaltic-rock } \\
\text { aquifers }\end{array}$ & VFPS & clptvfps1 & CLPTVFPS1-19 & WA & Franklin & $8 / 11 / 2014$ & 976.1 & NAVD 88 & Observation & 19 & 14 & 19 \\
\hline $\begin{array}{l}\text { Columbia } \\
\text { Plateau } \\
\text { basin-fill and } \\
\text { basaltic-rock } \\
\text { aquifers }\end{array}$ & VFPS & clptvfps1 & CLPTVFPS1-20 & WA & Franklin & $8 / 26 / 2014$ & 728.6 & NAVD 88 & Public supply & 747 & 22 & 703 \\
\hline $\begin{array}{l}\text { Columbia } \\
\text { Plateau } \\
\text { basin-fill and } \\
\text { basaltic-rock } \\
\text { aquifers }\end{array}$ & VFPS & clptvfps1 & CLPTVFPS1-21 & WA & Franklin & $8 / 27 / 2014$ & 927 & NAVD 88 & Public supply & 1,000 & 575 & 1,000 \\
\hline $\begin{array}{l}\text { Columbia } \\
\text { Plateau } \\
\text { basin-fill and } \\
\text { basaltic-rock } \\
\text { aquifers }\end{array}$ & VFPS & clptvfps1 & CLPTVFPS1-22 & WA & Franklin & $8 / 19 / 2014$ & 713.3 & NAVD 88 & Observation & 42.4 & 37 & 42 \\
\hline
\end{tabular}


Table 1. Information about wells that have environmental data included in this report.-Continued

[NAWQA, National Water-Quality Assessment; ID, identification; no., number; LS, land surface; $\mathrm{ft}$ bls, foot below land surface; lat., latitude, in degrees and minutes; long., longitude in degrees and minutes; PAS, principal aquifer study; FL, Florida; na, not available; NGVD 29, National Geodetic Vertical Datum of 1929; NAVD 88, North American Vertical Datum of 1988; ETN, enhanced trends network; TX, Texas; NM, New Mexico; LUS, land-use study; SC, South Carolina; FPS, flow-path study; MS, Mississippi; VFPS; vertical flow-path study; TN, Tennessee; OK, Oklahoma; AR, Arkansas; CA, California; MO, Missouri; KS, Kansas; MD, Maryland; MSS, modeling support study; DE, Delaware; DC, District of Columbia; MAS, major aquifer study; NV, Nevada; NY, New York; OH, Ohio; MI, Michigan; ID, Idaho; NH, New Hampshire; WI, Wisconsin; OR, Oregon; MN, Minnesota; WA, Washington]

\begin{tabular}{|c|c|c|c|c|c|c|c|c|c|c|c|c|}
\hline \multirow{2}{*}{$\begin{array}{l}\text { Principal and } \\
\text { regional and (or) } \\
\text { other aquifer } \\
\text { information }\end{array}$} & \multirow{2}{*}{$\begin{array}{l}\text { Network } \\
\text { type }\end{array}$} & \multirow{2}{*}{$\begin{array}{l}\text { Network } \\
\text { name }\end{array}$} & \multirow{2}{*}{$\begin{array}{l}\text { NAWQA well } \\
\text { ID no. }\end{array}$} & \multirow{2}{*}{ State } & \multirow{2}{*}{ County } & \multirow{2}{*}{ Sample date } & \multirow{2}{*}{$\begin{array}{l}\text { Altitude } \\
\text { LS }\end{array}$} & \multirow{2}{*}{$\begin{array}{l}\text { Altitude } \\
\text { datum }\end{array}$} & \multirow{2}{*}{ Water use } & \multirow{2}{*}{$\begin{array}{c}\text { Well } \\
\text { depth } \\
\text { (ft bls) }\end{array}$} & \multicolumn{2}{|c|}{$\begin{array}{c}\text { Depth to perforation } \\
\text { (ft bls) }\end{array}$} \\
\hline & & & & & & & & & & & Top & Bottom \\
\hline $\begin{array}{l}\text { Columbia } \\
\text { Plateau } \\
\text { basin-fill and } \\
\text { basaltic-rock } \\
\text { aquifers }\end{array}$ & VFPS & clptvfps 1 & CLPTVFPS1-23 & WA & Douglas & $7 / 7 / 2014$ & 640.5 & NAVD 88 & Public supply & 36 & 26 & 36 \\
\hline $\begin{array}{l}\text { Columbia } \\
\text { Plateau } \\
\text { basin-fill and } \\
\text { basaltic-rock } \\
\text { aquifers }\end{array}$ & VFPS & clptvfps 1 & CLPTVFPS1-24 & WA & Franklin & $8 / 25 / 2014$ & 926.9 & NAVD 88 & Public supply & 105 & 47 & 105 \\
\hline $\begin{array}{l}\text { Edwards-Trinity } \\
\text { aquifer system }\end{array}$ & ETN & edtretn1 & EDTRETN1-01 & $\mathrm{TX}$ & Bexar & $\begin{array}{l}\text { 11/12, } \\
\text { 12/17/2013; } \\
2 / 10,4 / 1 \\
7 / 17 \\
8 / 28,9 / 2 \\
\text { 11/24/2014; } \\
\text { 1/27, } 3 / 24 \\
5 / 20,6 / 4 \\
8 / 18,10 / 22 \\
12 / 9 / 2015 \\
3 / 10,5 / 9 \\
6 / 2,8 / 23 \\
10 / 19 / 2016\end{array}$ & 946 & NAVD 88 & Public supply & 550 & 317 & 550 \\
\hline
\end{tabular}


Table 1. Information about wells that have environmental data included in this report.-Continued

[NAWQA, National Water-Quality Assessment; ID, identification; no., number; LS, land surface; ft bls, foot below land surface; lat., latitude, in degrees and minutes; long., longitude in degrees and minutes; PAS, principal aquifer study; FL, Florida; na, not available; NGVD 29, National Geodetic Vertical Datum of 1929; NAVD 88, North American Vertical Datum of 1988; ETN, enhanced trends network; TX,

Texas; NM, New Mexico; LUS, land-use study; SC, South Carolina; FPS, flow-path study; MS, Mississippi; VFPS; vertical flow-path study; TN, Tennessee; OK, Oklahoma; AR, Arkansas; CA, California; MO, Missouri; KS, Kansas; MD, Maryland; MSS, modeling support study; DE, Delaware; DC, District of Columbia; MAS, major aquifer study; NV, Nevada; NY, New York; OH, Ohio; MI, Michigan; ID, Idaho;

NH, New Hampshire; WI, Wisconsin; OR, Oregon; MN, Minnesota; WA, Washington]

\begin{tabular}{|c|c|c|c|c|c|c|c|c|c|c|c|c|}
\hline \multirow{2}{*}{$\begin{array}{l}\text { Principal and } \\
\text { regional and (or) } \\
\text { other aquifer } \\
\text { information }\end{array}$} & \multirow{2}{*}{$\begin{array}{l}\text { Network } \\
\text { type }\end{array}$} & \multirow{2}{*}{$\begin{array}{l}\text { Network } \\
\text { name }\end{array}$} & \multirow{2}{*}{$\begin{array}{l}\text { NAWOA well } \\
\text { ID no. }\end{array}$} & \multirow{2}{*}{ State } & \multirow{2}{*}{ County } & \multirow{2}{*}{ Sample date } & \multirow{2}{*}{$\begin{array}{c}\text { Altitude } \\
\text { LS }\end{array}$} & \multirow{2}{*}{$\begin{array}{l}\text { Altitude } \\
\text { datum }\end{array}$} & \multirow{2}{*}{ Water use } & \multirow{2}{*}{$\begin{array}{c}\text { Well } \\
\text { depth } \\
\text { (ft bls) }\end{array}$} & \multicolumn{2}{|c|}{$\begin{array}{c}\text { Depth to perforation } \\
\text { (ft bls) }\end{array}$} \\
\hline & & & & & & & & & & & Top & Bottom \\
\hline $\begin{array}{l}\text { Edwards-Trinity } \\
\text { aquifer system }\end{array}$ & ETN & edtretn 1 & EDTRETN1-02 & TX & Bexar & $\begin{array}{l}\text { 11/13, } \\
\text { 12/16/2013; } \\
\text { 2/11, 3/31, } \\
\text { 6/2, 7/16, } 9 / 3 \\
\text { 11/25/2014; } \\
\text { 1/26, 3/25, } \\
\text { 5/15, 6/3 } \\
\text { 8/19, 10/21, } \\
\text { 12/7/2015; } \\
3 / 9,4 / 21 \\
6 / 1,8 / 22 \\
10 / 18 / 2016\end{array}$ & 975 & NGVD 29 & Observation & 300 & 220 & 300 \\
\hline $\begin{array}{l}\text { Edwards-Trinity } \\
\text { aquifer system }\end{array}$ & ETN & edtretn 1 & EDTRETN1-03 & TX & Bexar & $\begin{array}{l}\text { 11/14, } \\
\text { 12/17/2013; } \\
\text { 2/10, 4/1, } \\
\text { 6/3, 7/16, } 9 / 2 \\
\text { 11/24/2014; } \\
\text { 1/27, 3/26, } \\
\text { 5/27, 6/4, } \\
\text { 8/18, 10/22, } \\
\text { 12/8/2015; } \\
3 / 10,5 / 13 \\
\text { 6/2, 8/23, } \\
10 / 19 / 2016\end{array}$ & 585 & NAVD 88 & Public supply & 1,500 & 1,320 & 1,550 \\
\hline $\begin{array}{l}\text { Glacial aquifer } \\
\text { and New } \\
\text { England } \\
\text { crystalline- } \\
\text { rock aquifers }\end{array}$ & ETN & negxetn1 & NEGXETN1-01 & $\mathrm{NH}$ & Rockingham & $\begin{array}{c}1 / 13,3 / 11,5 / 24 \\
7 / 20,9 / 30 \\
11 / 16 / 2016\end{array}$ & 60 & NGVD 29 & Public supply & 83 & 73 & 83 \\
\hline $\begin{array}{l}\text { Glacial aquifer } \\
\text { and New } \\
\text { England } \\
\text { crystalline- } \\
\text { rock aquifers }\end{array}$ & ETN & negxetn 1 & NEGXETN1-02 & $\mathrm{NH}$ & Rockingham & $\begin{array}{c}1 / 13,3 / 11,5 / 23 \\
7 / 13,9 / 30 \\
11 / 16 / 2016\end{array}$ & 80 & NGVD 29 & Public supply & 492 & 88 & 492 \\
\hline
\end{tabular}


Table 1. Information about wells that have environmental data included in this report.-Continued

[NAWQA, National Water-Quality Assessment; ID, identification; no., number; LS, land surface; ft bls, foot below land surface; lat., latitude, in degrees and minutes; long., longitude in degrees and minutes; PAS, principal aquifer study; FL, Florida; na, not available; NGVD 29, National Geodetic Vertical Datum of 1929; NAVD 88, North American Vertical Datum of 1988; ETN, enhanced trends network; TX, Texas; NM, New Mexico; LUS, land-use study; SC, South Carolina; FPS, flow-path study; MS, Mississippi; VFPS; vertical flow-path study; TN, Tennessee; OK, Oklahoma; AR, Arkansas; CA, California; MO, Missouri; KS, Kansas; MD, Maryland; MSS, modeling support study; DE, Delaware; DC, District of Columbia; MAS, major aquifer study; NV, Nevada; NY, New York; OH, Ohio; MI, Michigan; ID, Idaho; NH, New Hampshire; WI, Wisconsin; OR, Oregon; MN, Minnesota; WA, Washington]

\begin{tabular}{|c|c|c|c|c|c|c|c|c|c|c|c|c|}
\hline \multirow{2}{*}{$\begin{array}{l}\text { Principal and } \\
\text { regional and (or) } \\
\text { other aquifer } \\
\text { information }\end{array}$} & \multirow{2}{*}{$\begin{array}{l}\text { Network } \\
\text { type }\end{array}$} & \multirow{2}{*}{$\begin{array}{l}\text { Network } \\
\text { name }\end{array}$} & \multirow{2}{*}{$\begin{array}{l}\text { NAWQA well } \\
\text { ID no. }\end{array}$} & \multirow{2}{*}{ State } & \multirow{2}{*}{ County } & \multirow{2}{*}{ Sample date } & \multirow{2}{*}{$\begin{array}{l}\text { Altitude } \\
\text { LS }\end{array}$} & \multirow{2}{*}{$\begin{array}{l}\text { Altitude } \\
\text { datum }\end{array}$} & \multirow{2}{*}{ Water use } & \multirow{2}{*}{$\begin{array}{l}\text { Well } \\
\text { depth } \\
\text { (ft bls) }\end{array}$} & \multicolumn{2}{|c|}{$\begin{array}{c}\text { Depth to perforation } \\
\text { (ft bls) }\end{array}$} \\
\hline & & & & & & & & & & & Top & Bottom \\
\hline $\begin{array}{l}\text { Glacial aquifer } \\
\text { and New } \\
\text { England } \\
\text { crystalline- } \\
\text { rock aquifers }\end{array}$ & ETN & negxetn1 & NEGXETN1-03 & $\mathrm{NH}$ & Rockingham & $\begin{array}{c}1 / 13,3 / 9,5 / 25 \\
7 / 12,9 / 29 \\
11 / 17 / 2016\end{array}$ & 110 & NGVD 29 & Domestic & 176.3 & na & $176.3^{a}$ \\
\hline $\begin{array}{l}\text { Glacial aquifer } \\
\text { system }\end{array}$ & MSS & glacmss 1 & GLACMSS1-01_SD & WI & Columbia & $8 / 21 / 2014$ & 824 & NGVD 29 & Public supply & 125 & 75 & 120 \\
\hline $\begin{array}{l}\text { Glacial aquifer } \\
\text { system }\end{array}$ & MSS & glacmss 1 & GLACMSS1-01_TD & WI & Marquette & $9 / 15 / 2014$ & 865 & NGVD 29 & Other & 199 & 180 & 200 \\
\hline $\begin{array}{l}\text { Glacial aquifer } \\
\text { system }\end{array}$ & MSS & glacmss 1 & GLACMSS1-01_TS & WI & Marquette & $8 / 20 / 2014$ & na & na & Observation & na & na & na \\
\hline $\begin{array}{l}\text { Glacial aquifer } \\
\text { system }\end{array}$ & MSS & glacmss 1 & GLACMSS1-02_SD & WI & Waupaca & $8 / 12 / 2014$ & na & na & Public supply & 141 & na & $141^{\mathrm{a}}$ \\
\hline $\begin{array}{l}\text { Glacial aquifer } \\
\text { system }\end{array}$ & MSS & glacmss 1 & GLACMSS1-02_TS & WI & Waushara & $8 / 20 / 2014$ & na & na & Observation & na & na & na \\
\hline $\begin{array}{l}\text { Glacial aquifer } \\
\text { system }\end{array}$ & MSS & glacmss 1 & GLACMSS1-02a_PD & WI & Portage & $8 / 11 / 2014$ & na & na & Public supply & 140 & na & $140^{\mathrm{a}}$ \\
\hline $\begin{array}{l}\text { Glacial aquifer } \\
\text { system }\end{array}$ & MSS & glacmss 1 & GLACMSS1-02a_PS & WI & Portage & $8 / 12 / 2014$ & 1,090 & NAVD 88 & Domestic & 63 & na & $63^{\mathrm{a}}$ \\
\hline $\begin{array}{l}\text { Glacial aquifer } \\
\text { system }\end{array}$ & MSS & glacmss 1 & GLACMSS1-02b_PD & WI & Waupaca & $8 / 11 / 2014$ & 844.5 & NGVD 29 & Public supply & 84 & 59 & 84 \\
\hline $\begin{array}{l}\text { Glacial aquifer } \\
\text { system }\end{array}$ & MSS & glacmss 1 & GLACMSS1-02b_PS & WI & Waupaca & $9 / 16 / 2014$ & 833 & NAVD 88 & Other & 45 & na & $45^{\mathrm{a}}$ \\
\hline $\begin{array}{l}\text { Glacial aquifer } \\
\text { system }\end{array}$ & MSS & glacmss 1 & GLACMSS1-03_SD & WI & Shawano & $8 / 13 / 2014$ & 1,160 & NGVD 29 & Public supply & 52 & 42.5 & 52.6 \\
\hline $\begin{array}{l}\text { Glacial aquifer } \\
\text { system }\end{array}$ & MSS & glacmss 1 & GLACMSS1-03_TD & WI & Shawano & $9 / 24 / 2014$ & 1,026 & NAVD 88 & Public supply & 88 & na & $88^{\mathrm{a}}$ \\
\hline $\begin{array}{l}\text { Glacial aquifer } \\
\text { system }\end{array}$ & MSS & glacmss 1 & GLACMSS1-03_TS & WI & Shawano & $9 / 22 / 2014$ & 1,287 & NAVD 88 & Other & 61 & na & $61^{\mathrm{a}}$ \\
\hline $\begin{array}{l}\text { Glacial aquifer } \\
\text { system }\end{array}$ & MSS & glacmss 1 & GLACMSS1-04_SD & WI & Forest & $8 / 13 / 2014$ & 1,637 & NGVD 29 & Public supply & 90 & 70 & 90 \\
\hline
\end{tabular}


Table 1. Information about wells that have environmental data included in this report.-Continued

[NAWQA, National Water-Quality Assessment; ID, identification; no., number; LS, land surface; $\mathrm{ft}$ bls, foot below land surface; lat., latitude, in degrees and minutes; long., longitude in degrees and minutes; PAS, principal aquifer study; FL, Florida; na, not available; NGVD 29, National Geodetic Vertical Datum of 1929; NAVD 88, North American Vertical Datum of 1988; ETN, enhanced trends network; TX,

Texas; NM, New Mexico; LUS, land-use study; SC, South Carolina; FPS, flow-path study; MS, Mississippi; VFPS; vertical flow-path study; TN, Tennessee; OK, Oklahoma; AR, Arkansas; CA, California; MO, Missouri; KS, Kansas; MD, Maryland; MSS, modeling support study; DE, Delaware; DC, District of Columbia; MAS, major aquifer study; NV, Nevada; NY, New York; OH, Ohio; MI, Michigan; ID, Idaho;

NH, New Hampshire; WI, Wisconsin; OR, Oregon; MN, Minnesota; WA, Washington]

\begin{tabular}{|c|c|c|c|c|c|c|c|c|c|c|c|c|}
\hline \multirow{2}{*}{$\begin{array}{l}\text { Principal and } \\
\text { regional and (or) } \\
\text { other aquifer } \\
\text { information }\end{array}$} & \multirow{2}{*}{$\begin{array}{l}\text { Network } \\
\text { type }\end{array}$} & \multirow{2}{*}{$\begin{array}{l}\text { Network } \\
\text { name }\end{array}$} & \multirow{2}{*}{$\begin{array}{l}\text { NAWQA well } \\
\text { ID no. }\end{array}$} & \multirow{2}{*}{ State } & \multirow{2}{*}{ County } & \multirow{2}{*}{ Sample date } & \multirow{2}{*}{$\begin{array}{c}\text { Altitude } \\
\text { LS }\end{array}$} & \multirow{2}{*}{$\begin{array}{l}\text { Altitude } \\
\text { datum }\end{array}$} & \multirow{2}{*}{ Water use } & \multirow{2}{*}{$\begin{array}{c}\text { Well } \\
\text { depth } \\
\text { (ft bls) }\end{array}$} & \multicolumn{2}{|c|}{$\begin{array}{c}\text { Depth to perforation } \\
\text { (ft bls) }\end{array}$} \\
\hline & & & & & & & & & & & Top & Bottom \\
\hline $\begin{array}{l}\text { Glacial aquifer } \\
\text { system }\end{array}$ & MSS & glacmss 1 & GLACMSS1-04_TS & WI & Oconto & $9 / 22 / 2014$ & 1,348 & NAVD 88 & Domestic & 76 & na & $76^{\mathrm{a}}$ \\
\hline $\begin{array}{l}\text { Glacial aquifer } \\
\text { system }\end{array}$ & MSS & glacmss 1 & GLACMSS1-05_SD & WI & Langlade & $8 / 18 / 2014$ & 1,290 & NGVD 29 & Public supply & 95.3 & 68.7 & 95.3 \\
\hline $\begin{array}{l}\text { Glacial aquifer } \\
\text { system }\end{array}$ & MSS & glacmss 1 & GLACMSS1-05_TD & WI & Oconto & $9 / 22 / 2014$ & 1,362 & NAVD 88 & Domestic & 170 & na & $170^{\mathrm{a}}$ \\
\hline $\begin{array}{l}\text { Glacial aquifer } \\
\text { system }\end{array}$ & MSS & glacmss 1 & GLACMSS1-05_TS & WI & Langlade & $9 / 22 / 2014$ & 1,317 & NAVD 88 & Public supply & 54 & na & $54 \mathrm{a}$ \\
\hline $\begin{array}{l}\text { Glacial aquifer } \\
\text { system }\end{array}$ & MSS & glacmss 1 & GLACMSS1-06_PD & WI & Waupaca & $8 / 11 / 2014$ & 780 & NGVD 29 & Public supply & 90 & 70 & 90 \\
\hline $\begin{array}{l}\text { Glacial aquifer } \\
\text { system }\end{array}$ & MSS & glacmss 1 & GLACMSS1-06_PS & WI & Waupaca & $9 / 17 / 2014$ & 792 & NAVD 88 & Other & 120 & 116 & 120 \\
\hline $\begin{array}{l}\text { Glacial aquifer } \\
\text { system }\end{array}$ & MSS & glacmss 1 & GLACMSS1-06_SD & WI & Waupaca & $9 / 23 / 2014$ & 852 & NGVD 29 & Public supply & 172 & 147 & 172 \\
\hline $\begin{array}{l}\text { Glacial aquifer } \\
\text { system }\end{array}$ & MSS & glacmss 1 & GLACMSS1-06_TS & WI & Waupaca & $9 / 24 / 2014$ & 907 & NAVD 88 & Domestic & 80 & na & $80^{a}$ \\
\hline $\begin{array}{l}\text { Glacial aquifer } \\
\text { system }\end{array}$ & MSS & glacmss 1 & GLACMSS1-07_SD & WI & Waupaca & $8 / 19 / 2014$ & 760 & NGVD 29 & Public supply & 118 & 75 & 115 \\
\hline $\begin{array}{l}\text { Glacial aquifer } \\
\text { system }\end{array}$ & MSS & glacmss 1 & GLACMSS1-07_TS & WI & Waushara & $9 / 24 / 2014$ & 788 & NAVD 88 & Other & 68 & na & $68^{\mathrm{a}}$ \\
\hline $\begin{array}{l}\text { Glacial aquifer } \\
\text { system }\end{array}$ & MSS & glacmss 1 & GLACMSS1-08_SD & WI & Outagamie & $9 / 16 / 2014$ & 800 & NGVD 29 & Public supply & 141 & 106 & 141 \\
\hline $\begin{array}{l}\text { Glacial aquifer } \\
\text { system }\end{array}$ & MSS & glacmss 1 & GLACMSS1-08_TS & WI & Shawano & $9 / 16 / 2014$ & 812 & NAVD 88 & Other & 62 & 54 & 62 \\
\hline $\begin{array}{l}\text { Glacial aquifer } \\
\text { system }\end{array}$ & MSS & glacmss 1 & GLACMSS1-09_SD & WI & Oconto & $8 / 19 / 2014$ & 810 & NGVD 29 & Public supply & 245 & 215 & 245 \\
\hline $\begin{array}{l}\text { Glacial aquifer } \\
\text { system }\end{array}$ & MSS & glacmss1 & GLACMSS1-09_TD & WI & Shawano & $9 / 23 / 2014$ & 838 & NGVD 29 & Public supply & 151 & 120 & 152 \\
\hline $\begin{array}{l}\text { Glacial aquifer } \\
\text { system }\end{array}$ & MSS & glacmss 1 & GLACMSS1-09_TS & WI & Oconto & $9 / 23 / 2014$ & 741 & NAVD 88 & Domestic & 53 & na & $53^{\mathrm{a}}$ \\
\hline
\end{tabular}


Table 1. Information about wells that have environmental data included in this report.-Continued

[NAWQA, National Water-Quality Assessment; ID, identification; no., number; LS, land surface; ft bls, foot below land surface; lat., latitude, in degrees and minutes; long., longitude in degrees and minutes; PAS, principal aquifer study; FL, Florida; na, not available; NGVD 29, National Geodetic Vertical Datum of 1929; NAVD 88, North American Vertical Datum of 1988; ETN, enhanced trends network; TX, Texas; NM, New Mexico; LUS, land-use study; SC, South Carolina; FPS, flow-path study; MS, Mississippi; VFPS; vertical flow-path study; TN, Tennessee; OK, Oklahoma; AR, Arkansas; CA, California; MO, Missouri; KS, Kansas; MD, Maryland; MSS, modeling support study; DE, Delaware; DC, District of Columbia; MAS, major aquifer study; NV, Nevada; NY, New York; OH, Ohio; MI, Michigan; ID, Idaho; NH, New Hampshire; WI, Wisconsin; OR, Oregon; MN, Minnesota; WA, Washington]

\begin{tabular}{|c|c|c|c|c|c|c|c|c|c|c|c|c|}
\hline \multirow{2}{*}{$\begin{array}{l}\text { Principal and } \\
\text { regional and (or) } \\
\text { other aquifer } \\
\text { information }\end{array}$} & \multirow{2}{*}{$\begin{array}{l}\text { Network } \\
\text { type }\end{array}$} & \multirow{2}{*}{$\begin{array}{c}\text { Network } \\
\text { name }\end{array}$} & \multirow{2}{*}{$\begin{array}{l}\text { NAWQA well } \\
\text { ID no. }\end{array}$} & \multirow{2}{*}{ State } & \multirow{2}{*}{ County } & \multirow{2}{*}{ Sample date } & \multirow{2}{*}{$\begin{array}{c}\text { Altitude } \\
\text { LS }\end{array}$} & \multirow{2}{*}{$\begin{array}{l}\text { Altitude } \\
\text { datum }\end{array}$} & \multirow{2}{*}{ Water use } & \multirow{2}{*}{$\begin{array}{c}\text { Well } \\
\text { depth } \\
\text { (ft bls) }\end{array}$} & \multicolumn{2}{|c|}{$\begin{array}{c}\text { Depth to perforation } \\
\text { (ft bls) }\end{array}$} \\
\hline & & & & & & & & & & & Top & Bottom \\
\hline $\begin{array}{l}\text { Glacial aquifer } \\
\text { system }\end{array}$ & MSS & glacmss 1 & GLACMSS1-10_SD & WI & Marinette & $8 / 18 / 2014$ & 697 & NAVD 88 & Public supply & 51.5 & na & $51.5^{\mathrm{a}}$ \\
\hline $\begin{array}{l}\text { Glacial aquifer } \\
\text { system }\end{array}$ & MSS & glacmss 1 & GLACMSS1-10_TS & WI & Oconto & $8 / 18 / 2014$ & na & na & Observation & na & na & na \\
\hline $\begin{array}{l}\text { Glacial aquifer } \\
\text { system }\end{array}$ & MAS & lerisus1 & LERISUS1-01 & MI & Livingston & $8 / 4 / 2016$ & 951 & NGVD 29 & Domestic & 103 & 99 & 103 \\
\hline $\begin{array}{l}\text { Glacial aquifer } \\
\text { system }\end{array}$ & MAS & lerisus1 & LERISUS1-02 & MI & Livingston & $8 / 4 / 2016$ & 987 & NGVD 29 & Domestic & 79 & 72 & 76 \\
\hline $\begin{array}{l}\text { Glacial aquifer } \\
\text { system }\end{array}$ & MAS & lerisus1 & LERISUS1-03 & MI & Oakland & $8 / 8 / 2016$ & 943 & NGVD 29 & Domestic & 72 & 60 & 70 \\
\hline $\begin{array}{l}\text { Glacial aquifer } \\
\text { system }\end{array}$ & MAS & lerisus1 & LERISUS1-04 & MI & Lenawee & $8 / 22 / 2016$ & 808 & NGVD 29 & Domestic & 158 & 155 & 158 \\
\hline $\begin{array}{l}\text { Glacial aquifer } \\
\text { system }\end{array}$ & MAS & lerisus1 & LERISUS1-05 & MI & Hillsdale & $8 / 22 / 2016$ & 900 & NAVD 88 & Domestic & 58 & 54 & 58 \\
\hline $\begin{array}{l}\text { Glacial aquifer } \\
\text { system }\end{array}$ & MAS & lerisus1 & LERISUS1-06 & MI & Hillsdale & $8 / 23 / 2016$ & 1,048 & NGVD 29 & Domestic & 90 & 86 & 90 \\
\hline $\begin{array}{l}\text { Glacial aquifer } \\
\text { system }\end{array}$ & MAS & lerisus1 & LERISUS1-07 & MI & Hillsdale & $8 / 23 / 2016$ & 922 & NGVD 29 & Domestic & 91 & 88 & 91 \\
\hline $\begin{array}{l}\text { Glacial aquifer } \\
\text { system }\end{array}$ & MAS & lerisus1 & LERISUS1-08 & MI & Lenawee & $8 / 24 / 2016$ & 865 & NGVD 29 & Domestic & 87 & 82 & 86 \\
\hline $\begin{array}{l}\text { Glacial aquifer } \\
\text { system }\end{array}$ & MAS & lerisus1 & LERISUS1-09 & MI & Lenawee & $8 / 24 / 2016$ & 863 & NGVD 29 & Domestic & 80 & 72 & 80 \\
\hline $\begin{array}{l}\text { Glacial aquifer } \\
\text { system }\end{array}$ & MAS & lerisus1 & LERISUS1-10 & MI & St. Clair & $9 / 6 / 2016$ & 788 & NGVD 29 & Domestic & 127 & 123 & 127 \\
\hline $\begin{array}{l}\text { Glacial aquifer } \\
\text { system }\end{array}$ & MAS & lerisus1 & LERISUS1-11 & MI & Sanilac & $9 / 6 / 2016$ & 814 & NGVD 29 & Domestic & 54 & 50 & 54 \\
\hline $\begin{array}{l}\text { Glacial aquifer } \\
\text { system }\end{array}$ & MAS & lerisus1 & LERISUS1-12 & MI & Lapeer & $9 / 6 / 2016$ & 833 & NAVD 88 & Domestic & 100 & 96 & 100 \\
\hline $\begin{array}{l}\text { Glacial aquifer } \\
\text { system }\end{array}$ & MAS & lerisus1 & LERISUS1-13 & MI & Oakland & 9/7/2016 & 815 & NGVD 29 & Domestic & 73 & 69 & 73 \\
\hline
\end{tabular}


Table 1. Information about wells that have environmental data included in this report.-Continued

[NAWQA, National Water-Quality Assessment; ID, identification; no., number; LS, land surface; ft bls, foot below land surface; lat., latitude, in degrees and minutes; long., longitude in degrees and minutes; PAS, principal aquifer study; FL, Florida; na, not available; NGVD 29, National Geodetic Vertical Datum of 1929; NAVD 88, North American Vertical Datum of 1988; ETN, enhanced trends network; TX,

Texas; NM, New Mexico; LUS, land-use study; SC, South Carolina; FPS, flow-path study; MS, Mississippi; VFPS; vertical flow-path study; TN, Tennessee; OK, Oklahoma; AR, Arkansas; CA, California; MO, Missouri; KS, Kansas; MD, Maryland; MSS, modeling support study; DE, Delaware; DC, District of Columbia; MAS, major aquifer study; NV, Nevada; NY, New York; OH, Ohio; MI, Michigan; ID, Idaho;

NH, New Hampshire; WI, Wisconsin; OR, Oregon; MN, Minnesota; WA, Washington]

\begin{tabular}{|c|c|c|c|c|c|c|c|c|c|c|c|c|}
\hline \multirow{2}{*}{$\begin{array}{l}\text { Principal and } \\
\text { regional and (or) } \\
\text { other aquifer } \\
\text { information }\end{array}$} & \multirow{2}{*}{$\begin{array}{l}\text { Network } \\
\text { type }\end{array}$} & \multirow{2}{*}{$\begin{array}{l}\text { Network } \\
\text { name }\end{array}$} & \multirow{2}{*}{$\begin{array}{l}\text { NAWQA well } \\
\text { ID no. }\end{array}$} & \multirow{2}{*}{ State } & \multirow{2}{*}{ County } & \multirow{2}{*}{ Sample date } & \multirow{2}{*}{$\begin{array}{c}\text { Altitude } \\
\text { LS }\end{array}$} & \multirow{2}{*}{$\begin{array}{l}\text { Altitude } \\
\text { datum }\end{array}$} & \multirow{2}{*}{ Water use } & \multirow{2}{*}{$\begin{array}{c}\text { Well } \\
\text { depth } \\
\text { (ft bls) }\end{array}$} & \multicolumn{2}{|c|}{$\begin{array}{c}\text { Depth to perforation } \\
\text { (ft bls) }\end{array}$} \\
\hline & & & & & & & & & & & Top & Bottom \\
\hline $\begin{array}{l}\text { Glacial aquifer } \\
\text { system }\end{array}$ & MAS & lerisus1 & LERISUS1-14 & MI & Lapeer & $9 / 8 / 2016$ & 862 & NGVD 29 & Domestic & 100 & 92 & 100 \\
\hline $\begin{array}{l}\text { Glacial aquifer } \\
\text { system }\end{array}$ & MAS & lerisus1 & LERISUS1-15 & MI & Macomb & $9 / 8 / 2016$ & 630 & NGVD 29 & Domestic & 35 & 31 & 35 \\
\hline $\begin{array}{l}\text { Glacial aquifer } \\
\text { system }\end{array}$ & MAS & lerisus1 & LERISUS1-16 & MI & Oakland & $9 / 12 / 2016$ & 1,117 & NGVD 29 & Domestic & 120 & 116 & 120 \\
\hline $\begin{array}{l}\text { Glacial aquifer } \\
\text { system }\end{array}$ & MAS & lerisus 1 & LERISUS1-17 & MI & Sanilac & $9 / 12 / 2016$ & 812 & NGVD 29 & Domestic & 205 & 201 & 205 \\
\hline $\begin{array}{l}\text { Glacial aquifer } \\
\text { system }\end{array}$ & MAS & lerisus 1 & LERISUS1-18 & MI & Oakland & $9 / 12 / 2016$ & 1,020 & NGVD 29 & Domestic & 170 & 162 & 170 \\
\hline $\begin{array}{l}\text { Glacial aquifer } \\
\text { system }\end{array}$ & MAS & lerisus 1 & LERISUS1-19 & MI & Washtenaw & $9 / 13 / 2016$ & 995 & NGVD 29 & Domestic & 92 & 88 & 92 \\
\hline $\begin{array}{l}\text { Glacial aquifer } \\
\text { system }\end{array}$ & MAS & lerisus1 & LERISUS1-20 & MI & Washtenaw & $9 / 13 / 2016$ & 921 & NGVD 29 & Domestic & 100 & 92 & 96 \\
\hline $\begin{array}{l}\text { Glacial aquifer } \\
\text { system }\end{array}$ & MAS & lerisus1 & LERISUS1-21 & $\mathrm{OH}$ & Williams & $8 / 23 / 2016$ & 927 & NGVD 29 & Domestic & 70 & 62 & 67 \\
\hline $\begin{array}{l}\text { Glacial aquifer } \\
\text { system }\end{array}$ & MAS & lerisus1 & LERISUS1-22 & $\mathrm{OH}$ & Williams & $8 / 23 / 2016$ & 980 & NGVD 29 & Domestic & 82 & 78 & 82 \\
\hline $\begin{array}{l}\text { Glacial aquifer } \\
\text { system }\end{array}$ & MAS & lerisus 1 & LERISUS1-23 & $\mathrm{OH}$ & Williams & $8 / 24 / 2016$ & 912 & NGVD 29 & Domestic & 70 & 67 & 70 \\
\hline $\begin{array}{l}\text { Glacial aquifer } \\
\text { system }\end{array}$ & MAS & lerisus1 & LERISUS1-24 & $\mathrm{OH}$ & Williams & $8 / 24 / 2016$ & 863 & NGVD 29 & Domestic & 121 & 118 & 121 \\
\hline $\begin{array}{l}\text { Glacial aquifer } \\
\text { system }\end{array}$ & MAS & pugtsus1 & PUGTSUS1-01 & WA & Pierce & $8 / 22 / 2016$ & 1,450 & NGVD 29 & Domestic & 78 & 78 & $78^{a}$ \\
\hline $\begin{array}{l}\text { Glacial aquifer } \\
\text { system }\end{array}$ & MAS & pugtsus 1 & PUGTSUS1-02 & WA & Pierce & $8 / 22 / 2016$ & 480 & NGVD 29 & Domestic & 78 & 78 & $78^{\mathrm{a}}$ \\
\hline $\begin{array}{l}\text { Glacial aquifer } \\
\text { system }\end{array}$ & MAS & pugtsus1 & PUGTSUS1-03 & WA & Pierce & $8 / 23 / 2016$ & 360 & NGVD 29 & Domestic & 40 & 40 & $40^{\mathrm{a}}$ \\
\hline $\begin{array}{l}\text { Glacial aquifer } \\
\text { system }\end{array}$ & MAS & pugtsus1 & PUGTSUS1-04 & WA & Pierce & $8 / 23 / 2016$ & 360 & NGVD 29 & Domestic & 100 & 100 & $100^{\mathrm{a}}$ \\
\hline
\end{tabular}


Table 1. Information about wells that have environmental data included in this report.-Continued

[NAWQA, National Water-Quality Assessment; ID, identification; no., number; LS, land surface; ft bls, foot below land surface; lat., latitude, in degrees and minutes; long., longitude in degrees and minutes; PAS, principal aquifer study; FL, Florida; na, not available; NGVD 29, National Geodetic Vertical Datum of 1929; NAVD 88, North American Vertical Datum of 1988; ETN, enhanced trends network; TX, Texas; NM, New Mexico; LUS, land-use study; SC, South Carolina; FPS, flow-path study; MS, Mississippi; VFPS; vertical flow-path study; TN, Tennessee; OK, Oklahoma; AR, Arkansas; CA, California; MO, Missouri; KS, Kansas; MD, Maryland; MSS, modeling support study; DE, Delaware; DC, District of Columbia; MAS, major aquifer study; NV, Nevada; NY, New York; OH, Ohio; MI, Michigan; ID, Idaho; NH, New Hampshire; WI, Wisconsin; OR, Oregon; MN, Minnesota; WA, Washington]

\begin{tabular}{|c|c|c|c|c|c|c|c|c|c|c|c|c|}
\hline \multirow{2}{*}{$\begin{array}{l}\text { Principal and } \\
\text { regional and (or) } \\
\text { other aquifer } \\
\text { information }\end{array}$} & \multirow{2}{*}{$\begin{array}{l}\text { Network } \\
\text { type }\end{array}$} & \multirow{2}{*}{$\begin{array}{l}\text { Network } \\
\text { name }\end{array}$} & \multirow{2}{*}{$\begin{array}{l}\text { NAWQA well } \\
\text { ID no. }\end{array}$} & \multirow{2}{*}{ State } & \multirow{2}{*}{ County } & \multirow{2}{*}{ Sample date } & \multirow{2}{*}{$\begin{array}{l}\text { Altitude } \\
\text { LS }\end{array}$} & \multirow{2}{*}{$\begin{array}{l}\text { Altitude } \\
\text { datum }\end{array}$} & \multirow{2}{*}{ Water use } & \multirow{2}{*}{$\begin{array}{l}\text { Well } \\
\text { depth } \\
\text { (ft bls) }\end{array}$} & \multicolumn{2}{|c|}{$\begin{array}{c}\text { Depth to perforation } \\
\text { (ft bls) }\end{array}$} \\
\hline & & & & & & & & & & & Top & Bottom \\
\hline $\begin{array}{l}\text { Glacial aquifer } \\
\text { system }\end{array}$ & MAS & pugtsus1 & PUGTSUS1-05 & WA & Snohomish & $8 / 25 / 2016$ & 1,040 & NGVD 29 & Domestic & 186 & 182 & 186 \\
\hline $\begin{array}{l}\text { Glacial aquifer } \\
\text { system }\end{array}$ & MAS & pugtsus1 & PUGTSUS1-06 & WA & Snohomish & $8 / 25 / 2016$ & 220 & NGVD 29 & Domestic & 71 & 70 & $71 \mathrm{a}$ \\
\hline $\begin{array}{l}\text { Glacial aquifer } \\
\text { system }\end{array}$ & MAS & pugtsus 1 & PUGTSUS1-07 & WA & Pierce & $9 / 6 / 2016$ & 720 & NGVD 29 & Domestic & 40 & 40 & $40^{\mathrm{a}}$ \\
\hline $\begin{array}{l}\text { Glacial aquifer } \\
\text { system }\end{array}$ & MAS & pugtsus1 & PUGTSUS1-08 & WA & Thurston & $9 / 6 / 2016$ & 430 & NGVD 29 & Domestic & 58 & 58 & $58 \mathrm{a}$ \\
\hline $\begin{array}{l}\text { Glacial aquifer } \\
\text { system }\end{array}$ & MAS & pugtsus1 & PUGTSUS1-09 & WA & Thurston & $9 / 13 / 2016$ & 14 & NGVD 29 & Domestic & 72 & 67 & 72 \\
\hline $\begin{array}{l}\text { Glacial aquifer } \\
\text { system }\end{array}$ & MAS & pugtsus1 & PUGTSUS1-10 & WA & Thurston & $9 / 13 / 2016$ & 210 & NGVD 29 & Domestic & 68 & 62 & 64 \\
\hline $\begin{array}{l}\text { Glacial aquifer } \\
\text { system }\end{array}$ & MAS & pugtsus1 & PUGTSUS1-11 & WA & Snohomish & $9 / 15 / 2016$ & 230 & NGVD 29 & Domestic & 70 & 65 & 70 \\
\hline $\begin{array}{l}\text { Glacial aquifer } \\
\text { system }\end{array}$ & MAS & pugtsus1 & PUGTSUS1-12 & WA & Whatcom & $9 / 21 / 2016$ & 144 & NGVD 29 & Domestic & 29 & 24 & 29 \\
\hline $\begin{array}{l}\text { Glacial aquifer } \\
\text { system }\end{array}$ & MAS & pugtsus1 & PUGTSUS1-13 & WA & Whatcom & $9 / 21 / 2016$ & 65 & NGVD 29 & Domestic & 22.5 & 17.5 & 22.5 \\
\hline $\begin{array}{l}\text { Glacial aquifer } \\
\text { system }\end{array}$ & MAS & pugtsus1 & PUGTSUS1-14 & WA & King & $11 / 1 / 2016$ & 320 & NGVD 29 & Domestic & 75 & 70 & 75 \\
\hline $\begin{array}{l}\text { Glacial aquifer } \\
\text { system }\end{array}$ & MAS & pugtsus1 & PUGTSUS1-15 & WA & Snohomish & $11 / 8 / 2016$ & 240 & NGVD 29 & Domestic & 42 & 32 & 42 \\
\hline $\begin{array}{l}\text { Glacial aquifer } \\
\text { system }\end{array}$ & MAS & pugtsus1 & PUGTSUS1-16 & WA & Kitsap & $11 / 14 / 2016$ & 67 & NAVD 88 & Domestic & 85 & 82 & 85 \\
\hline $\begin{array}{l}\text { Glacial aquifer } \\
\text { system }\end{array}$ & MAS & pugtsus1 & PUGTSUS1-17 & WA & Whatcom & $11 / 16 / 2016$ & 60 & NGVD 29 & Domestic & 40 & 35 & 40 \\
\hline $\begin{array}{l}\text { Glacial aquifer } \\
\text { system }\end{array}$ & MAS & pugtsus1 & PUGTSUS1-18 & WA & Whatcom & $11 / 16 / 2016$ & 130 & NGVD 29 & Domestic & 98 & 98 & $98^{\mathrm{a}}$ \\
\hline $\begin{array}{l}\text { Glacial aquifer } \\
\text { system }\end{array}$ & MAS & pugtsus1 & PUGTSUS1-19 & WA & King & $11 / 17 / 2016$ & 346 & NAVD 88 & Public supply & 230 & 190 & 230 \\
\hline
\end{tabular}


Table 1. Information about wells that have environmental data included in this report.-Continued

[NAWQA, National Water-Quality Assessment; ID, identification; no., number; LS, land surface; $\mathrm{ft}$ bls, foot below land surface; lat., latitude, in degrees and minutes; long., longitude in degrees and minutes; PAS, principal aquifer study; FL, Florida; na, not available; NGVD 29, National Geodetic Vertical Datum of 1929; NAVD 88, North American Vertical Datum of 1988; ETN, enhanced trends network; TX,

Texas; NM, New Mexico; LUS, land-use study; SC, South Carolina; FPS, flow-path study; MS, Mississippi; VFPS; vertical flow-path study; TN, Tennessee; OK, Oklahoma; AR, Arkansas; CA, California; MO, Missouri; KS, Kansas; MD, Maryland; MSS, modeling support study; DE, Delaware; DC, District of Columbia; MAS, major aquifer study; NV, Nevada; NY, New York; OH, Ohio; MI, Michigan; ID, Idaho;

NH, New Hampshire; WI, Wisconsin; OR, Oregon; MN, Minnesota; WA, Washington]

\begin{tabular}{|c|c|c|c|c|c|c|c|c|c|c|c|c|}
\hline \multirow{2}{*}{$\begin{array}{l}\text { Principal and } \\
\text { regional and (or) } \\
\text { other aquifer } \\
\text { information }\end{array}$} & \multirow{2}{*}{$\begin{array}{l}\text { Network } \\
\text { type }\end{array}$} & \multirow{2}{*}{$\begin{array}{l}\text { Network } \\
\text { name }\end{array}$} & \multirow{2}{*}{$\begin{array}{l}\text { NAWQA well } \\
\text { ID no. }\end{array}$} & \multirow{2}{*}{ State } & \multirow{2}{*}{ County } & \multirow{2}{*}{ Sample date } & \multirow{2}{*}{$\begin{array}{c}\text { Altitude } \\
\text { LS }\end{array}$} & \multirow{2}{*}{$\begin{array}{l}\text { Altitude } \\
\text { datum }\end{array}$} & \multirow{2}{*}{ Water use } & \multirow{2}{*}{$\begin{array}{l}\text { Well } \\
\text { depth } \\
\text { (ft bls) }\end{array}$} & \multicolumn{2}{|c|}{$\begin{array}{c}\text { Depth to perforation } \\
\text { (ft bls) }\end{array}$} \\
\hline & & & & & & & & & & & Top & Bottom \\
\hline $\begin{array}{l}\text { Glacial aquifer } \\
\text { system }\end{array}$ & MAS & pugtsus1 & PUGTSUS1-20 & WA & Kitsap & $11 / 21 / 2016$ & 304 & NAVD 88 & Domestic & 300 & na & $300^{a}$ \\
\hline $\begin{array}{l}\text { Glacial aquifer } \\
\text { system }\end{array}$ & MAS & pugtsus1 & PUGTSUS1-21 & WA & Mason & $11 / 21 / 2016$ & 262 & NAVD 88 & Domestic & 309 & na & $309^{a}$ \\
\hline $\begin{array}{l}\text { Glacial aquifer } \\
\text { system }\end{array}$ & MAS & pugtsus 1 & PUGTSUS1-22 & WA & Mason & $11 / 22 / 2016$ & 480 & NAVD 88 & Domestic & 60 & 55.5 & 60 \\
\hline $\begin{array}{l}\text { Glacial aquifer } \\
\text { system }\end{array}$ & MAS & pugtsus1 & PUGTSUS1-23 & WA & Mason & $11 / 22 / 2016$ & 227 & NAVD 88 & Domestic & 180 & 120 & 180 \\
\hline $\begin{array}{l}\text { Glacial aquifer } \\
\text { system }\end{array}$ & MAS & pugtsus1 & PUGTSUS1-24 & WA & Kitsap & $11 / 28 / 2016$ & 219 & NAVD 88 & Other & 277 & 272 & 277 \\
\hline $\begin{array}{l}\text { Glacial aquifer } \\
\text { system }\end{array}$ & MAS & pugtsus1 & PUGTSUS1-25 & WA & King & $11 / 29 / 2016$ & 532 & NGVD 29 & Public supply & 85 & 75 & 85 \\
\hline $\begin{array}{l}\text { Glacial aquifer } \\
\text { system }\end{array}$ & MAS & pugtsus1 & PUGTSUS1-26 & WA & King & $12 / 5 / 2016$ & 47 & NGVD 29 & Public supply & 92 & 82 & 92 \\
\hline $\begin{array}{l}\text { Glacial aquifer } \\
\text { system }\end{array}$ & MAS & pugtsus1 & PUGTSUS1-27 & WA & King & $12 / 5 / 2016$ & 40 & NGVD 29 & Public supply & 82 & 44 & 82 \\
\hline $\begin{array}{l}\text { Glacial aquifer } \\
\text { system }\end{array}$ & MAS & pugtsus1 & PUGTSUS1-28 & WA & Pierce & $12 / 7 / 2016$ & 261 & NAVD 88 & Public supply & 112 & 55 & 107 \\
\hline $\begin{array}{l}\text { Glacial aquifer } \\
\text { system }\end{array}$ & MAS & pugtsus1 & PUGTSUS1-29 & WA & Thurston & $12 / 7 / 2016$ & 29 & NAVD 88 & Public supply & 170.6 & 130 & 159.6 \\
\hline $\begin{array}{l}\text { Glacial aquifer } \\
\text { system }\end{array}$ & LUS & umislusrc1 & UMISLUSRC1-01 & $\mathrm{MN}$ & Anoka & $7 / 11 / 2016$ & 857.9 & NAVD 88 & Observation & 14 & 9 & 14 \\
\hline $\begin{array}{l}\text { Glacial aquifer } \\
\text { system }\end{array}$ & LUS & umislusrc1 & UMISLUSRC1-02 & $\mathrm{MN}$ & Anoka & $7 / 12 / 2016$ & 856.9 & NGVD 29 & Observation & 18 & 13 & 18 \\
\hline $\begin{array}{l}\text { Glacial aquifer } \\
\text { system }\end{array}$ & LUS & umislusrc1 & UMISLUSRC1-03 & $\mathrm{MN}$ & Anoka & $7 / 12 / 2016$ & 894.7 & NAVD 88 & Observation & 15 & 10 & 15 \\
\hline $\begin{array}{l}\text { Glacial aquifer } \\
\text { system }\end{array}$ & LUS & umislusrc1 & UMISLUSRC1-04 & $\mathrm{MN}$ & Anoka & $7 / 13 / 2016$ & 879.1 & NAVD 88 & Observation & 20.8 & 15.8 & 20.8 \\
\hline $\begin{array}{l}\text { Glacial aquifer } \\
\text { system }\end{array}$ & LUS & umislusrc1 & UMISLUSRC1-05 & $\mathrm{MN}$ & Anoka & $7 / 13 / 2016$ & 877.4 & NGVD 29 & Observation & 20 & 15 & 20 \\
\hline
\end{tabular}


Table 1. Information about wells that have environmental data included in this report.-Continued

[NAWQA, National Water-Quality Assessment; ID, identification; no., number; LS, land surface; ft bls, foot below land surface; lat., latitude, in degrees and minutes; long., longitude in degrees and minutes; PAS, principal aquifer study; FL, Florida; na, not available; NGVD 29, National Geodetic Vertical Datum of 1929; NAVD 88, North American Vertical Datum of 1988; ETN, enhanced trends network; TX, Texas; NM, New Mexico; LUS, land-use study; SC, South Carolina; FPS, flow-path study; MS, Mississippi; VFPS; vertical flow-path study; TN, Tennessee; OK, Oklahoma; AR, Arkansas; CA, California; MO, Missouri; KS, Kansas; MD, Maryland; MSS, modeling support study; DE, Delaware; DC, District of Columbia; MAS, major aquifer study; NV, Nevada; NY, New York; OH, Ohio; MI, Michigan; ID, Idaho; NH, New Hampshire; WI, Wisconsin; OR, Oregon; MN, Minnesota; WA, Washington]

\begin{tabular}{|c|c|c|c|c|c|c|c|c|c|c|c|c|}
\hline \multirow{2}{*}{$\begin{array}{l}\text { Principal and } \\
\text { regional and (or) } \\
\text { other aquifer } \\
\text { information }\end{array}$} & \multirow{2}{*}{$\begin{array}{l}\text { Network } \\
\text { type }\end{array}$} & \multirow{2}{*}{$\begin{array}{l}\text { Network } \\
\text { name }\end{array}$} & \multirow{2}{*}{$\begin{array}{l}\text { NAWQA well } \\
\text { ID no. }\end{array}$} & \multirow{2}{*}{ State } & \multirow{2}{*}{ County } & \multirow{2}{*}{ Sample date } & \multirow{2}{*}{$\begin{array}{l}\text { Altitude } \\
\text { LS }\end{array}$} & \multirow{2}{*}{$\begin{array}{c}\text { Altitude } \\
\text { datum }\end{array}$} & \multirow{2}{*}{ Water use } & \multirow{2}{*}{$\begin{array}{l}\text { Well } \\
\text { depth } \\
\text { (ft bls) }\end{array}$} & \multicolumn{2}{|c|}{$\begin{array}{c}\text { Depth to perforation } \\
\text { (ft bls) }\end{array}$} \\
\hline & & & & & & & & & & & Top & Bottom \\
\hline $\begin{array}{l}\text { Glacial aquifer } \\
\text { system }\end{array}$ & LUS & umislusrc1 & UMISLUSRC1-06 & $\mathrm{MN}$ & Anoka & $7 / 14 / 2016$ & 854.1 & NAVD 88 & Observation & 12.2 & 7.2 & 12.2 \\
\hline $\begin{array}{l}\text { Glacial aquifer } \\
\text { system }\end{array}$ & LUS & umislusrc1 & UMISLUSRC1-07 & $\mathrm{MN}$ & Hennepin & $7 / 14 / 2016$ & 849.1 & NGVD 29 & Observation & 9 & 4 & 9 \\
\hline $\begin{array}{l}\text { Glacial aquifer } \\
\text { system }\end{array}$ & LUS & umislusrc1 & UMISLUSRC1-08 & $\mathrm{MN}$ & Hennepin & $8 / 1 / 2016$ & 867.9 & NGVD 29 & Observation & 13.5 & 8.5 & 13.5 \\
\hline $\begin{array}{l}\text { Glacial aquifer } \\
\text { system }\end{array}$ & LUS & umislusrc1 & UMISLUSRC1-09 & $\mathrm{MN}$ & Hennepin & $8 / 2 / 2016$ & 858.4 & NGVD 29 & Observation & 24 & 19 & 24 \\
\hline $\begin{array}{l}\text { Glacial aquifer } \\
\text { system }\end{array}$ & LUS & umislusrc1 & UMISLUSRC1-10 & $\mathrm{MN}$ & Hennepin & $8 / 2 / 2016$ & 910.8 & NGVD 29 & Observation & 28.5 & 24 & 28.5 \\
\hline $\begin{array}{l}\text { Glacial aquifer } \\
\text { system }\end{array}$ & LUS & umislusrc1 & UMISLUSRC1-11 & $\mathrm{MN}$ & Anoka & $8 / 3 / 2016$ & 862 & NGVD 29 & Observation & 19 & 14 & 19 \\
\hline $\begin{array}{l}\text { Glacial aquifer } \\
\text { system }\end{array}$ & LUS & umislusrc1 & UMISLUSRC1-12 & $\mathrm{MN}$ & Hennepin & $8 / 4 / 2016$ & 872.8 & NGVD 29 & Observation & 18 & 13 & 18 \\
\hline $\begin{array}{l}\text { Glacial aquifer } \\
\text { system }\end{array}$ & LUS & umislusrc1 & UMISLUSRC1-13 & $\mathrm{MN}$ & Hennepin & $8 / 15 / 2016$ & 841.8 & NAVD 88 & Observation & 16 & 11 & 16 \\
\hline $\begin{array}{l}\text { Glacial aquifer } \\
\text { system }\end{array}$ & LUS & umislusrc1 & UMISLUSRC1-14 & $\mathrm{MN}$ & Hennepin & $8 / 15 / 2016$ & 866.6 & NGVD 29 & Observation & 22.5 & 17.5 & 22.5 \\
\hline $\begin{array}{l}\text { Glacial aquifer } \\
\text { system }\end{array}$ & LUS & umislusrc1 & UMISLUSRC1-15 & $\mathrm{MN}$ & Hennepin & $8 / 16 / 2016$ & 845.8 & NAVD 88 & Observation & 15 & 10 & 15 \\
\hline $\begin{array}{l}\text { Glacial aquifer } \\
\text { system }\end{array}$ & LUS & umislusrc1 & UMISLUSRC1-16 & $\mathrm{MN}$ & Hennepin & $8 / 17 / 2016$ & 880.6 & NAVD 88 & Observation & 26 & 16 & 26 \\
\hline $\begin{array}{l}\text { Glacial aquifer } \\
\text { system }\end{array}$ & LUS & umislusrc1 & UMISLUSRC1-17 & $\mathrm{MN}$ & Hennepin & $8 / 18 / 2016$ & 872.3 & NAVD 88 & Observation & 25.4 & 20.4 & 25.1 \\
\hline $\begin{array}{l}\text { Glacial aquifer } \\
\text { system }\end{array}$ & LUS & umislusrc1 & UMISLUSRC1-18 & $\mathrm{MN}$ & Hennepin & $8 / 22 / 2016$ & 864.9 & NAVD 88 & Observation & 18 & 13 & 18 \\
\hline $\begin{array}{l}\text { Glacial aquifer } \\
\text { system }\end{array}$ & LUS & umislusrc1 & UMISLUSRC1-19 & $\mathrm{MN}$ & Hennepin & $8 / 22 / 2016$ & 857.7 & NGVD 29 & Observation & 19 & 14 & 19 \\
\hline $\begin{array}{l}\text { Glacial aquifer } \\
\text { system }\end{array}$ & LUS & umislusrc1 & UMISLUSRC1-20 & $\mathrm{MN}$ & Anoka & $8 / 23 / 2016$ & 895.7 & NGVD 29 & Observation & 24.5 & 19.5 & 24.5 \\
\hline
\end{tabular}


Table 1. Information about wells that have environmental data included in this report.-Continued

[NAWQA, National Water-Quality Assessment; ID, identification; no., number; LS, land surface; ft bls, foot below land surface; lat., latitude, in degrees and minutes; long., longitude in degrees and minutes; PAS, principal aquifer study; FL, Florida; na, not available; NGVD 29, National Geodetic Vertical Datum of 1929; NAVD 88, North American Vertical Datum of 1988; ETN, enhanced trends network; TX,

Texas; NM, New Mexico; LUS, land-use study; SC, South Carolina; FPS, flow-path study; MS, Mississippi; VFPS; vertical flow-path study; TN, Tennessee; OK, Oklahoma; AR, Arkansas; CA, California; MO, Missouri; KS, Kansas; MD, Maryland; MSS, modeling support study; DE, Delaware; DC, District of Columbia; MAS, major aquifer study; NV, Nevada; NY, New York; OH, Ohio; MI, Michigan; ID, Idaho;

NH, New Hampshire; WI, Wisconsin; OR, Oregon; MN, Minnesota; WA, Washington]

\begin{tabular}{|c|c|c|c|c|c|c|c|c|c|c|c|c|}
\hline \multirow{2}{*}{$\begin{array}{l}\text { Principal and } \\
\text { regional and (or) } \\
\text { other aquifer } \\
\text { information }\end{array}$} & \multirow{2}{*}{$\begin{array}{l}\text { Network } \\
\text { type }\end{array}$} & \multirow{2}{*}{$\begin{array}{l}\text { Network } \\
\text { name }\end{array}$} & \multirow{2}{*}{$\begin{array}{l}\text { NAWQA well } \\
\text { ID no. }\end{array}$} & \multirow{2}{*}{ State } & \multirow{2}{*}{ County } & \multirow{2}{*}{ Sample date } & \multirow{2}{*}{$\begin{array}{l}\text { Altitude } \\
\text { LS }\end{array}$} & \multirow{2}{*}{$\begin{array}{l}\text { Altitude } \\
\text { datum }\end{array}$} & \multirow{2}{*}{ Water use } & \multirow{2}{*}{$\begin{array}{c}\text { Well } \\
\text { depth } \\
\text { (ft bls) }\end{array}$} & \multicolumn{2}{|c|}{$\begin{array}{c}\text { Depth to perforation } \\
\text { (ft bls) }\end{array}$} \\
\hline & & & & & & & & & & & Top & Bottom \\
\hline $\begin{array}{l}\text { Glacial aquifer } \\
\text { system }\end{array}$ & LUS & umislusrc1 & UMISLUSRC1-21 & $\mathrm{MN}$ & Hennepin & $8 / 23 / 2016$ & 844.1 & NGVD 29 & Observation & 17 & 12 & 17 \\
\hline $\begin{array}{l}\text { Glacial aquifer } \\
\text { system }\end{array}$ & LUS & umislusrc1 & UMISLUSRC1-22 & $\mathrm{MN}$ & Hennepin & $8 / 23 / 2016$ & 863.3 & NGVD 29 & Observation & 23 & 18 & 23 \\
\hline $\begin{array}{l}\text { Glacial aquifer } \\
\text { system }\end{array}$ & LUS & umislusrc1 & UMISLUSRC1-23 & $\mathrm{MN}$ & Hennepin & $8 / 24 / 2016$ & 868.1 & NGVD 29 & Observation & 24.3 & 19.3 & 24.3 \\
\hline $\begin{array}{l}\text { Glacial aquifer } \\
\text { system }\end{array}$ & LUS & umislusrc1 & UMISLUSRC1-24 & $\mathrm{MN}$ & Hennepin & $8 / 25 / 2016$ & 876.2 & NGVD 29 & Observation & 18 & 13 & 18 \\
\hline $\begin{array}{l}\text { Glacial aquifer } \\
\text { system }\end{array}$ & LUS & umislusrc1 & UMISLUSRC1-25 & $\mathrm{MN}$ & Hennepin & $8 / 25 / 2016$ & 851 & NGVD 29 & Observation & 10 & 5 & 10 \\
\hline $\begin{array}{l}\text { Glacial aquifer } \\
\text { system }\end{array}$ & LUS & umislusrc1 & UMISLUSRC1-26 & $\mathrm{MN}$ & Hennepin & $8 / 25 / 2016$ & 849.6 & NGVD 29 & Observation & 15 & 10 & 15 \\
\hline $\begin{array}{l}\text { Glacial aquifer } \\
\text { system }\end{array}$ & LUS & umislusrc1 & UMISLUSRC1-27 & $\mathrm{MN}$ & Hennepin & $8 / 29 / 2016$ & 875.2 & NGVD 29 & Observation & 29 & 24 & 29 \\
\hline $\begin{array}{l}\text { Glacial aquifer } \\
\text { system }\end{array}$ & LUS & umislusrc1 & UMISLUSRC1-28 & $\mathrm{MN}$ & Hennepin & $8 / 29 / 2016$ & 850.4 & NGVD 29 & Observation & 33.5 & 28.5 & 33.5 \\
\hline $\begin{array}{l}\text { Glacial aquifer } \\
\text { system }\end{array}$ & LUS & umislusrc1 & UMISLUSRC1-29 & $\mathrm{MN}$ & Anoka & $8 / 30 / 2016$ & 867.9 & NGVD 29 & Observation & 13 & 8 & 13 \\
\hline $\begin{array}{l}\text { Glacial aquifer } \\
\text { system }\end{array}$ & LUS & umislusrc1 & UMISLUSRC1-30 & $\mathrm{MN}$ & Hennepin & 9/8/2016 & 857.9 & NGVD 29 & Observation & 9 & 4 & 9 \\
\hline $\begin{array}{l}\text { Glacial aquifer } \\
\text { system }\end{array}$ & $\begin{array}{l}\text { LUS/ } \\
\text { VFPS }\end{array}$ & $\begin{array}{l}\text { lerilusrc1/ } \\
\text { glacvfps1 }\end{array}$ & $\begin{array}{l}\text { LERILUSRC1-01/ } \\
\text { GLACVFPS1-11 }\end{array}$ & MI & Livingston & $8 / 1 / 2016$ & 895 & NGVD 29 & Observation & 25 & 20.1 & 24.6 \\
\hline $\begin{array}{l}\text { Glacial aquifer } \\
\text { system }\end{array}$ & $\begin{array}{l}\text { LUS/ } \\
\text { VFPS }\end{array}$ & $\begin{array}{l}\text { lerilusrc1/ } \\
\text { glacvfps1 }\end{array}$ & $\begin{array}{l}\text { LERILUSRC1-02/ } \\
\text { GLACVFPS1-09 }\end{array}$ & MI & Livingston & $8 / 1 / 2016$ & 880 & NGVD 29 & Observation & 26.5 & 21.2 & 25.7 \\
\hline $\begin{array}{l}\text { Glacial aquifer } \\
\text { system }\end{array}$ & $\begin{array}{l}\text { LUS/ } \\
\text { VFPS }\end{array}$ & $\begin{array}{l}\text { lerilusrc1/ } \\
\text { glacvfps1 }\end{array}$ & $\begin{array}{l}\text { LERILUSRC1-03/ } \\
\text { GLACVFPS1-07 }\end{array}$ & MI & Livingston & $8 / 2 / 2016$ & 905 & NGVD 29 & Observation & 15.4 & 10.5 & 15 \\
\hline $\begin{array}{l}\text { Glacial aquifer } \\
\text { system }\end{array}$ & $\begin{array}{l}\text { LUS/ } \\
\text { VFPS }\end{array}$ & $\begin{array}{l}\text { lerilusrc1/ } \\
\text { glacvfps1 }\end{array}$ & $\begin{array}{l}\text { LERILUSRC1-04/ } \\
\text { GLACVFPS1-06 }\end{array}$ & MI & Washtenaw & $8 / 2 / 2016$ & 870 & NAVD 88 & Observation & 28.5 & 23.2 & 28.2 \\
\hline
\end{tabular}


Table 1. Information about wells that have environmental data included in this report.-Continued

[NAWQA, National Water-Quality Assessment; ID, identification; no., number; LS, land surface; ft bls, foot below land surface; lat., latitude, in degrees and minutes; long., longitude in degrees and minutes; PAS, principal aquifer study; FL, Florida; na, not available; NGVD 29, National Geodetic Vertical Datum of 1929; NAVD 88, North American Vertical Datum of 1988; ETN, enhanced trends network; TX, Texas; NM, New Mexico; LUS, land-use study; SC, South Carolina; FPS, flow-path study; MS, Mississippi; VFPS; vertical flow-path study; TN, Tennessee; OK, Oklahoma; AR, Arkansas; CA, California; MO, Missouri; KS, Kansas; MD, Maryland; MSS, modeling support study; DE, Delaware; DC, District of Columbia; MAS, major aquifer study; NV, Nevada; NY, New York; OH, Ohio; MI, Michigan; ID, Idaho; NH, New Hampshire; WI, Wisconsin; OR, Oregon; MN, Minnesota; WA, Washington]

\begin{tabular}{|c|c|c|c|c|c|c|c|c|c|c|c|c|}
\hline \multirow{2}{*}{$\begin{array}{l}\text { Principal and } \\
\text { regional and (or) } \\
\text { other aquifer } \\
\text { information }\end{array}$} & \multirow{2}{*}{$\begin{array}{l}\text { Network } \\
\text { type }\end{array}$} & \multirow{2}{*}{$\begin{array}{l}\text { Network } \\
\text { name }\end{array}$} & \multirow{2}{*}{$\begin{array}{l}\text { NAWQA well } \\
\text { ID no. }\end{array}$} & \multirow{2}{*}{ State } & \multirow{2}{*}{ County } & \multirow{2}{*}{ Sample date } & \multirow{2}{*}{$\begin{array}{l}\text { Altitude } \\
\text { LS }\end{array}$} & \multirow{2}{*}{$\begin{array}{l}\text { Altitude } \\
\text { datum }\end{array}$} & \multirow{2}{*}{ Water use } & \multirow{2}{*}{$\begin{array}{l}\text { Well } \\
\text { depth } \\
\text { (ft bls) }\end{array}$} & \multicolumn{2}{|c|}{$\begin{array}{c}\text { Depth to perforation } \\
\text { (ft bls) }\end{array}$} \\
\hline & & & & & & & & & & & Top & Bottom \\
\hline $\begin{array}{l}\text { Glacial aquifer } \\
\text { system }\end{array}$ & $\begin{array}{l}\text { LUS/ } \\
\text { VFPS }\end{array}$ & $\begin{array}{l}\text { lerilusrc1/ } \\
\text { glacvfps1 }\end{array}$ & $\begin{array}{l}\text { LERILUSRC1-05/ } \\
\text { GLACVFPS1-18 }\end{array}$ & MI & Livingston & $8 / 3 / 2016$ & 925 & NGVD 29 & Observation & 21 & 16.2 & 20.7 \\
\hline $\begin{array}{l}\text { Glacial aquifer } \\
\text { system }\end{array}$ & $\begin{array}{l}\text { LUS/ } \\
\text { VFPS }\end{array}$ & $\begin{array}{l}\text { lerilusrc1/ } \\
\text { glacvfps1 }\end{array}$ & $\begin{array}{l}\text { LERILUSRC1-06/ } \\
\text { GLACVFPS1-19 }\end{array}$ & MI & Livingston & $8 / 3 / 2016$ & 935 & NGVD 29 & Observation & 16.3 & 11.5 & 16 \\
\hline $\begin{array}{l}\text { Glacial aquifer } \\
\text { system }\end{array}$ & $\begin{array}{l}\text { LUS/ } \\
\text { VFPS }\end{array}$ & $\begin{array}{l}\text { lerilusrc1/ } \\
\text { glacvfps1 }\end{array}$ & $\begin{array}{l}\text { LERILUSRC1-07/ } \\
\text { GLACVFPS1-20 }\end{array}$ & MI & Livingston & $8 / 3 / 2016$ & 995 & NGVD 29 & Observation & 30.4 & 25.5 & 30 \\
\hline $\begin{array}{l}\text { Glacial aquifer } \\
\text { system }\end{array}$ & $\begin{array}{l}\text { LUS/ } \\
\text { VFPS }\end{array}$ & $\begin{array}{l}\text { lerilusrc1/ } \\
\text { glacvfps1 }\end{array}$ & $\begin{array}{l}\text { LERILUSRC1-08/ } \\
\text { GLACVFPS1-13 }\end{array}$ & MI & Oakland & 8/9/2016 & 915 & NGVD 29 & Observation & 10.5 & 5.7 & 10.2 \\
\hline $\begin{array}{l}\text { Glacial aquifer } \\
\text { system }\end{array}$ & $\begin{array}{l}\text { LUS/ } \\
\text { VFPS }\end{array}$ & $\begin{array}{l}\text { lerilusrc1/ } \\
\text { glacvfps 1 }\end{array}$ & $\begin{array}{l}\text { LERILUSRC1-09/ } \\
\text { GLACVFPS1-25 }\end{array}$ & MI & Oakland & $8 / 10 / 2016$ & 935 & NGVD 29 & Observation & 20.1 & 15.2 & 19.7 \\
\hline $\begin{array}{l}\text { Glacial aquifer } \\
\text { system }\end{array}$ & $\begin{array}{l}\text { LUS/ } \\
\text { VFPS }\end{array}$ & $\begin{array}{l}\text { lerilusrc1/ } \\
\text { glacvfps1 }\end{array}$ & $\begin{array}{l}\text { LERILUSRC1-10/ } \\
\text { GLACVFPS1-22 }\end{array}$ & MI & Oakland & $8 / 11 / 2016$ & 940 & NAVD 88 & Observation & 49 & 40.1 & 48.2 \\
\hline $\begin{array}{l}\text { Glacial aquifer } \\
\text { system }\end{array}$ & $\begin{array}{l}\text { LUS/ } \\
\text { VFPS }\end{array}$ & $\begin{array}{l}\text { lerilusrc1/ } \\
\text { glacvfps1 }\end{array}$ & $\begin{array}{l}\text { LERILUSRC1-11/ } \\
\text { GLACVFPS1-16 }\end{array}$ & MI & Livingston & $8 / 15 / 2016$ & 935 & NGVD 29 & Observation & 40.9 & 36.1 & 40.6 \\
\hline $\begin{array}{l}\text { Glacial aquifer } \\
\text { system }\end{array}$ & $\begin{array}{l}\text { LUS/ } \\
\text { VFPS }\end{array}$ & $\begin{array}{l}\text { lerilusrc1/ } \\
\text { glacvfps1 }\end{array}$ & $\begin{array}{l}\text { LERILUSRC1-12/ } \\
\text { GLACVFPS1-27 }\end{array}$ & MI & Livingston & $8 / 15 / 2016$ & 1,010 & NGVD 29 & Observation & 68.5 & 63.6 & 68.1 \\
\hline $\begin{array}{l}\text { Glacial aquifer } \\
\text { system }\end{array}$ & $\begin{array}{l}\text { LUS/ } \\
\text { VFPS }\end{array}$ & $\begin{array}{l}\text { lerilusrc1/ } \\
\text { glacvfps1 }\end{array}$ & $\begin{array}{l}\text { LERILUSRC1-13/ } \\
\text { GLACVFPS1-33 }\end{array}$ & MI & Oakland & $8 / 16 / 2016$ & 990 & NGVD 29 & Observation & 39.8 & 35 & 39.5 \\
\hline $\begin{array}{l}\text { Glacial aquifer } \\
\text { system }\end{array}$ & $\begin{array}{l}\text { LUS/ } \\
\text { VFPS }\end{array}$ & $\begin{array}{l}\text { lerilusrc1/ } \\
\text { glacvfps1 }\end{array}$ & $\begin{array}{l}\text { LERILUSRC1-14/ } \\
\text { GLACVFPS1-29 }\end{array}$ & MI & Oakland & $8 / 16 / 2016$ & 1,035 & NGVD 29 & Observation & 32.1 & 27.3 & 31.8 \\
\hline $\begin{array}{l}\text { Glacial aquifer } \\
\text { system }\end{array}$ & $\begin{array}{l}\text { LUS/ } \\
\text { VFPS }\end{array}$ & $\begin{array}{l}\text { lerilusrc1/ } \\
\text { glacvfps1 }\end{array}$ & $\begin{array}{l}\text { LERILUSRC1-15/ } \\
\text { GLACVFPS1-31 }\end{array}$ & MI & Oakland & $8 / 16 / 2016$ & 1,025 & NGVD 29 & Observation & 22.1 & 17.3 & 21.8 \\
\hline $\begin{array}{l}\text { Glacial aquifer } \\
\text { system }\end{array}$ & $\begin{array}{l}\text { LUS/ } \\
\text { VFPS }\end{array}$ & $\begin{array}{l}\text { lerilusrc1/ } \\
\text { glacvfps1 }\end{array}$ & $\begin{array}{l}\text { LERILUSRC1-16/ } \\
\text { GLACVFPS1-35 }\end{array}$ & MI & Oakland & $8 / 17 / 2016$ & 1,045 & NGVD 29 & Observation & 41.7 & 36.9 & 41.4 \\
\hline $\begin{array}{l}\text { Glacial aquifer } \\
\text { system }\end{array}$ & $\begin{array}{l}\text { LUS/ } \\
\text { VFPS }\end{array}$ & $\begin{array}{l}\text { lerilusrc1/ } \\
\text { glacvfps1 }\end{array}$ & $\begin{array}{l}\text { LERILUSRC1-17/ } \\
\text { GLACVFPS1-39 }\end{array}$ & MI & Oakland & $8 / 17 / 2016$ & 1,040 & NGVD 29 & Observation & 18.1 & 13.3 & 17.8 \\
\hline
\end{tabular}


Table 1. Information about wells that have environmental data included in this report.-Continued

[NAWQA, National Water-Quality Assessment; ID, identification; no., number; LS, land surface; $\mathrm{ft}$ bls, foot below land surface; lat., latitude, in degrees and minutes; long., longitude in degrees and minutes; PAS, principal aquifer study; FL, Florida; na, not available; NGVD 29, National Geodetic Vertical Datum of 1929; NAVD 88, North American Vertical Datum of 1988; ETN, enhanced trends network; TX,

Texas; NM, New Mexico; LUS, land-use study; SC, South Carolina; FPS, flow-path study; MS, Mississippi; VFPS; vertical flow-path study; TN, Tennessee; OK, Oklahoma; AR, Arkansas; CA, California; MO, Missouri; KS, Kansas; MD, Maryland; MSS, modeling support study; DE, Delaware; DC, District of Columbia; MAS, major aquifer study; NV, Nevada; NY, New York; OH, Ohio; MI, Michigan; ID, Idaho;

NH, New Hampshire; WI, Wisconsin; OR, Oregon; MN, Minnesota; WA, Washington]

\begin{tabular}{|c|c|c|c|c|c|c|c|c|c|c|c|c|}
\hline \multirow{2}{*}{$\begin{array}{l}\text { Principal and } \\
\text { regional and (or) } \\
\text { other aquifer } \\
\text { information }\end{array}$} & \multirow{2}{*}{$\begin{array}{l}\text { Network } \\
\text { type }\end{array}$} & \multirow{2}{*}{$\begin{array}{l}\text { Network } \\
\text { name }\end{array}$} & \multirow{2}{*}{$\begin{array}{l}\text { NAWQA well } \\
\text { ID no. }\end{array}$} & \multirow{2}{*}{ State } & \multirow{2}{*}{ County } & \multirow{2}{*}{ Sample date } & \multirow{2}{*}{$\begin{array}{l}\text { Altitude } \\
\text { LS }\end{array}$} & \multirow{2}{*}{$\begin{array}{l}\text { Altitude } \\
\text { datum }\end{array}$} & \multirow{2}{*}{ Water use } & \multirow{2}{*}{$\begin{array}{c}\text { Well } \\
\text { depth } \\
\text { (ft bls) }\end{array}$} & \multicolumn{2}{|c|}{$\begin{array}{c}\text { Depth to perforation } \\
\text { (ft bls) }\end{array}$} \\
\hline & & & & & & & & & & & Top & Bottom \\
\hline $\begin{array}{l}\text { Glacial aquifer } \\
\text { system }\end{array}$ & $\begin{array}{l}\text { LUS/ } \\
\text { VFPS }\end{array}$ & $\begin{array}{l}\text { lerilusrc1/ } \\
\text { glacvfps1 }\end{array}$ & $\begin{array}{l}\text { LERILUSRC1-18/ } \\
\text { GLACVFPS1-42 }\end{array}$ & MI & Oakland & $8 / 18 / 2016$ & 1,045 & NGVD 29 & Observation & 16.7 & 11.9 & 16.4 \\
\hline $\begin{array}{l}\text { Glacial aquifer } \\
\text { system }\end{array}$ & $\begin{array}{l}\text { LUS/ } \\
\text { VFPS }\end{array}$ & $\begin{array}{l}\text { lerilusrc1/ } \\
\text { glacvfps1 }\end{array}$ & $\begin{array}{l}\text { LERILUSRC1-19/ } \\
\text { GLACVFPS1-38 }\end{array}$ & MI & Oakland & $9 / 7 / 2016$ & 1,055 & NAVD 88 & Observation & 39.9 & 34.4 & 39.4 \\
\hline $\begin{array}{l}\text { Glacial aquifer } \\
\text { system }\end{array}$ & $\begin{array}{l}\text { LUS/ } \\
\text { VFPS }\end{array}$ & $\begin{array}{l}\text { lerilusrc1/ } \\
\text { glacvfps1 }\end{array}$ & $\begin{array}{l}\text { LERILUSRC1-20/ } \\
\text { GLACVFPS1-34 }\end{array}$ & MI & Macomb & $9 / 7 / 2016$ & 840 & NAVD 88 & Observation & 30 & 25 & 30 \\
\hline $\begin{array}{l}\text { Glacial aquifer } \\
\text { system }\end{array}$ & $\begin{array}{l}\text { LUS/ } \\
\text { VFPS }\end{array}$ & $\begin{array}{l}\text { lerilusrc1/ } \\
\text { glacvfps1 }\end{array}$ & $\begin{array}{l}\text { LERILUSRC1-21/ } \\
\text { GLACVFPS1-37 }\end{array}$ & MI & Oakland & $9 / 7 / 2016$ & 995 & NGVD 29 & Observation & 16.6 & 11.8 & 16.3 \\
\hline $\begin{array}{l}\text { Glacial aquifer } \\
\text { system }\end{array}$ & $\begin{array}{l}\text { LUS/ } \\
\text { VFPS }\end{array}$ & $\begin{array}{l}\text { lerilusrc1/ } \\
\text { glacvfps1 }\end{array}$ & $\begin{array}{l}\text { LERILUSRC1-22/ } \\
\text { GLACVFPS1-23 }\end{array}$ & MI & Oakland & 9/13/2016 & 920 & NGVD 29 & Observation & 31.8 & 27 & 31.5 \\
\hline $\begin{array}{l}\text { Glacial aquifer } \\
\text { system }\end{array}$ & $\begin{array}{l}\text { LUS/ } \\
\text { VFPS }\end{array}$ & $\begin{array}{l}\text { lerilusrc1/ } \\
\text { glacvfps1 }\end{array}$ & $\begin{array}{l}\text { LERILUSRC1-23/ } \\
\text { GLACVFPS1-41 }\end{array}$ & MI & Oakland & $9 / 14 / 2016$ & 1,045 & NGVD 29 & Observation & 33.9 & 29 & 33.5 \\
\hline $\begin{array}{l}\text { Glacial aquifer } \\
\text { system }\end{array}$ & $\begin{array}{l}\text { LUS/ } \\
\text { VFPS }\end{array}$ & $\begin{array}{l}\text { lerilusrc1/ } \\
\text { glacvfps1 }\end{array}$ & $\begin{array}{l}\text { LERILUSRC1-24/ } \\
\text { GLACVFPS1-01 }\end{array}$ & MI & Washtenaw & $9 / 14 / 2016$ & 845 & NGVD 29 & Observation & 27.3 & 22.5 & 27 \\
\hline $\begin{array}{l}\text { Glacial aquifer } \\
\text { system }\end{array}$ & $\begin{array}{l}\text { LUS/ } \\
\text { VFPS }\end{array}$ & $\begin{array}{l}\text { lerilusrc1/ } \\
\text { glacvfps1 }\end{array}$ & $\begin{array}{l}\text { LERILUSRC1-25/ } \\
\text { GLACVFPS1-03 }\end{array}$ & MI & Washtenaw & $9 / 15 / 2016$ & 835 & NGVD 29 & Observation & 22 & 17.1 & 21.7 \\
\hline $\begin{array}{l}\text { Glacial aquifer } \\
\text { system }\end{array}$ & $\begin{array}{l}\text { LUS/ } \\
\text { VFPS }\end{array}$ & $\begin{array}{l}\text { lerilusrc1/ } \\
\text { glacvfps1 }\end{array}$ & $\begin{array}{l}\text { LERILUSRC1-26/ } \\
\text { GLACVFPS1-04 }\end{array}$ & MI & Washtenaw & $9 / 15 / 2016$ & 855 & NGVD 29 & Observation & 31.2 & 26.4 & 30.9 \\
\hline $\begin{array}{l}\text { Glacial aquifer } \\
\text { system }\end{array}$ & $\begin{array}{l}\text { LUS/ } \\
\text { VFPS }\end{array}$ & $\begin{array}{l}\text { lerilusrc1/ } \\
\text { glacvfps1 }\end{array}$ & $\begin{array}{l}\text { LERILUSRC1-27/ } \\
\text { GLACVFPS1-32 }\end{array}$ & MI & Oakland & $9 / 22 / 2016$ & 768 & NAVD 88 & Observation & 23 & 15 & 22.5 \\
\hline $\begin{array}{l}\text { Glacial aquifer } \\
\text { system }\end{array}$ & $\begin{array}{l}\text { LUS/ } \\
\text { VFPS }\end{array}$ & $\begin{array}{l}\text { lerilusrc1/ } \\
\text { glacvfps1 }\end{array}$ & $\begin{array}{l}\text { LERILUSRC1-28/ } \\
\text { GLACVFPS1-15 }\end{array}$ & MI & Oakland & $9 / 27 / 2016$ & 875 & NGVD 29 & Observation & 13.9 & 9.1 & 13.6 \\
\hline $\begin{array}{l}\text { Glacial aquifer } \\
\text { system }\end{array}$ & VFPS & glacvfps1 & GLACVFPS1-02 & MI & Washtenaw & $9 / 14 / 2016$ & 845 & NGVD 29 & Domestic & 138 & 134 & 138 \\
\hline $\begin{array}{l}\text { Glacial aquifer } \\
\text { system }\end{array}$ & VFPS & glacvfps 1 & GLACVFPS1-05 & MI & Washtenaw & $9 / 15 / 2016$ & 855 & NGVD 29 & Domestic & 145 & 140 & 145 \\
\hline $\begin{array}{l}\text { Glacial aquifer } \\
\text { system }\end{array}$ & VFPS & glacvfps1 & GLACVFPS1-08 & MI & Livingston & $8 / 2 / 2016$ & 910 & NGVD 29 & Domestic & 49 & 44 & 49 \\
\hline
\end{tabular}


Table 1. Information about wells that have environmental data included in this report.-Continued

[NAWQA, National Water-Quality Assessment; ID, identification; no., number; LS, land surface; ft bls, foot below land surface; lat., latitude, in degrees and minutes; long., longitude in degrees and minutes; PAS, principal aquifer study; FL, Florida; na, not available; NGVD 29, National Geodetic Vertical Datum of 1929; NAVD 88, North American Vertical Datum of 1988; ETN, enhanced trends network; TX, Texas; NM, New Mexico; LUS, land-use study; SC, South Carolina; FPS, flow-path study; MS, Mississippi; VFPS; vertical flow-path study; TN, Tennessee; OK, Oklahoma; AR, Arkansas; CA, California; MO, Missouri; KS, Kansas; MD, Maryland; MSS, modeling support study; DE, Delaware; DC, District of Columbia; MAS, major aquifer study; NV, Nevada; NY, New York; OH, Ohio; MI, Michigan; ID, Idaho; NH, New Hampshire; WI, Wisconsin; OR, Oregon; MN, Minnesota; WA, Washington]

\begin{tabular}{|c|c|c|c|c|c|c|c|c|c|c|c|c|}
\hline \multirow{2}{*}{$\begin{array}{l}\text { Principal and } \\
\text { regional and (or) } \\
\text { other aquifer } \\
\text { information }\end{array}$} & \multirow{2}{*}{$\begin{array}{l}\text { Network } \\
\text { type }\end{array}$} & \multirow{2}{*}{$\begin{array}{l}\text { Network } \\
\text { name }\end{array}$} & \multirow{2}{*}{$\begin{array}{l}\text { NAWQA well } \\
\text { ID no. }\end{array}$} & \multirow{2}{*}{ State } & \multirow{2}{*}{ County } & \multirow{2}{*}{ Sample date } & \multirow{2}{*}{$\begin{array}{l}\text { Altitude } \\
\text { LS }\end{array}$} & \multirow{2}{*}{$\begin{array}{l}\text { Altitude } \\
\text { datum }\end{array}$} & \multirow{2}{*}{ Water use } & \multirow{2}{*}{$\begin{array}{l}\text { Well } \\
\text { depth } \\
\text { (ft bls) }\end{array}$} & \multicolumn{2}{|c|}{$\begin{array}{c}\text { Depth to perforation } \\
\text { (ft bls) }\end{array}$} \\
\hline & & & & & & & & & & & Top & Bottom \\
\hline $\begin{array}{l}\text { Glacial aquifer } \\
\text { system }\end{array}$ & VFPS & glacvfps 1 & GLACVFPS1-10 & MI & Livingston & $8 / 1 / 2016$ & 880 & NGVD 29 & Domestic & 60 & 56 & 60 \\
\hline $\begin{array}{l}\text { Glacial aquifer } \\
\text { system }\end{array}$ & VFPS & glacvfps 1 & GLACVFPS1-12 & MI & Livingston & $8 / 2 / 2016$ & 895 & NGVD 29 & Domestic & 116 & 112 & 116 \\
\hline $\begin{array}{l}\text { Glacial aquifer } \\
\text { system }\end{array}$ & VFPS & glacvfps 1 & GLACVFPS1-14 & MI & Oakland & $8 / 9 / 2016$ & 915 & NGVD 29 & Domestic & 38 & 32 & 38 \\
\hline $\begin{array}{l}\text { Glacial aquifer } \\
\text { system }\end{array}$ & VFPS & glacvfps 1 & GLACVFPS1-17 & MI & Livingston & $8 / 15 / 2016$ & 935 & NGVD 29 & Domestic & 106 & 102 & 106 \\
\hline $\begin{array}{l}\text { Glacial aquifer } \\
\text { system }\end{array}$ & VFPS & glacvfps 1 & GLACVFPS1-21 & MI & Livingston & $8 / 3 / 2016$ & 995 & NGVD 29 & Domestic & 92 & 88 & 92 \\
\hline $\begin{array}{l}\text { Glacial aquifer } \\
\text { system }\end{array}$ & VFPS & glacvfps 1 & GLACVFPS1-24 & MI & Oakland & $9 / 13 / 2016$ & 920 & NGVD 29 & Domestic & 127 & 123 & 127 \\
\hline $\begin{array}{l}\text { Glacial aquifer } \\
\text { system }\end{array}$ & VFPS & glacvfps 1 & GLACVFPS1-26 & MI & Oakland & $8 / 10 / 2016$ & 933 & NAVD 88 & Domestic & 60 & 56 & 60 \\
\hline $\begin{array}{l}\text { Glacial aquifer } \\
\text { system }\end{array}$ & VFPS & glacvfps 1 & GLACVFPS1-28 & MI & Livingston & $8 / 15 / 2016$ & 1,000 & NGVD 29 & Domestic & 105 & 101 & 105 \\
\hline $\begin{array}{l}\text { Glacial aquifer } \\
\text { system }\end{array}$ & VFPS & glacvfps1 & GLACVFPS1-30 & MI & Oakland & $8 / 16 / 2016$ & 1,035 & NGVD 29 & Domestic & 90 & 80 & 90 \\
\hline $\begin{array}{l}\text { Glacial aquifer } \\
\text { system }\end{array}$ & VFPS & glacvfps1 & GLACVFPS1-36 & MI & Oakland & $8 / 17 / 2016$ & 1,035 & NGVD 29 & Domestic & 172 & 168 & 172 \\
\hline $\begin{array}{l}\text { Glacial aquifer } \\
\text { system }\end{array}$ & VFPS & glacvfps 1 & GLACVFPS $1-40$ & MI & Oakland & $8 / 17 / 2016$ & 1,040 & NGVD 29 & Domestic & 127 & 123 & 127 \\
\hline $\begin{array}{l}\text { Glacial aquifer } \\
\text { system }\end{array}$ & VFPS & glacvfps1 & GLACVFPS $1-43$ & MI & Oakland & $8 / 18 / 2016$ & 1,045 & NGVD 29 & Domestic & 84 & 80 & 84 \\
\hline $\begin{array}{l}\text { Glacial aquifer } \\
\text { system }\end{array}$ & $\begin{array}{l}\text { ETN/ } \\
\text { FPS }\end{array}$ & $\begin{array}{l}\text { glacetn1/ } \\
\text { glacfps1 }\end{array}$ & $\begin{array}{l}\text { GLACETN1-01/ } \\
\text { GLACFPS1-17 }\end{array}$ & WI & Portage & $\begin{array}{l}\text { 6/9/2014; } 2 / 17, \\
5 / 6,6 / 29,8 / 2, \\
9 / 5 / 2016\end{array}$ & $1,135.70$ & NAVD 88 & Observation & 83 & 80 & 83 \\
\hline $\begin{array}{l}\text { Glacial aquifer } \\
\text { system }\end{array}$ & $\begin{array}{l}\text { ETN/ } \\
\text { FPS }\end{array}$ & $\begin{array}{l}\text { glacetn1/ } \\
\text { glacfps1 }\end{array}$ & $\begin{array}{l}\text { GLACETN1-02/ } \\
\text { GLACFPS1-18 }\end{array}$ & WI & Portage & $\begin{array}{l}6 / 9 / 2014 ; 2 / 17, \\
5 / 6,6 / 29,8 / 2, \\
9 / 5 / 2016\end{array}$ & $1,135.70$ & NAVD 88 & Observation & 34.5 & 24.5 & 34.5 \\
\hline
\end{tabular}


Table 1. Information about wells that have environmental data included in this report.-Continued

[NAWQA, National Water-Quality Assessment; ID, identification; no., number; LS, land surface; ft bls, foot below land surface; lat., latitude, in degrees and minutes; long., longitude in degrees and minutes; PAS, principal aquifer study; FL, Florida; na, not available; NGVD 29, National Geodetic Vertical Datum of 1929; NAVD 88, North American Vertical Datum of 1988; ETN, enhanced trends network; TX,

Texas; NM, New Mexico; LUS, land-use study; SC, South Carolina; FPS, flow-path study; MS, Mississippi; VFPS; vertical flow-path study; TN, Tennessee; OK, Oklahoma; AR, Arkansas; CA, California; MO, Missouri; KS, Kansas; MD, Maryland; MSS, modeling support study; DE, Delaware; DC, District of Columbia; MAS, major aquifer study; NV, Nevada; NY, New York; OH, Ohio; MI, Michigan; ID, Idaho;

NH, New Hampshire; WI, Wisconsin; OR, Oregon; MN, Minnesota; WA, Washington]

\begin{tabular}{|c|c|c|c|c|c|c|c|c|c|c|c|c|}
\hline \multirow{2}{*}{$\begin{array}{l}\text { Principal and } \\
\text { regional and (or) } \\
\text { other aquifer } \\
\text { information }\end{array}$} & \multirow{2}{*}{$\begin{array}{l}\text { Network } \\
\text { type }\end{array}$} & \multirow{2}{*}{$\begin{array}{l}\text { Network } \\
\text { name }\end{array}$} & \multirow{2}{*}{$\begin{array}{l}\text { NAWOA well } \\
\text { ID no. }\end{array}$} & \multirow{2}{*}{ State } & \multirow{2}{*}{ County } & \multirow{2}{*}{ Sample date } & \multirow{2}{*}{$\begin{array}{l}\text { Altitude } \\
\text { LS }\end{array}$} & \multirow{2}{*}{$\begin{array}{l}\text { Altitude } \\
\text { datum }\end{array}$} & \multirow{2}{*}{ Water use } & \multirow{2}{*}{$\begin{array}{l}\text { Well } \\
\text { depth } \\
\text { (ft bls) }\end{array}$} & \multicolumn{2}{|c|}{$\begin{array}{c}\text { Depth to perforation } \\
\text { (ft bls) }\end{array}$} \\
\hline & & & & & & & & & & & Top & Bottom \\
\hline $\begin{array}{l}\text { Glacial aquifer } \\
\text { system }\end{array}$ & ETN & glacetn1 & GLACETN1-03 & WI & Sauk & $8 / 3 / 2016$ & 723.4 & NAVD 88 & Observation & 50.1 & 45.1 & 50.1 \\
\hline $\begin{array}{l}\text { Glacial aquifer } \\
\text { system }\end{array}$ & ETN & glacetn1 & GLACETN1-04 & WI & Sauk & $8 / 26 / 2016$ & 723.4 & NAVD 88 & Observation & 89 & 84 & 89 \\
\hline $\begin{array}{l}\text { Glacial aquifer } \\
\text { system }\end{array}$ & ETN & glacetn1 & GLACETN1-05 & WI & Sauk & $8 / 3 / 2016$ & 730 & NAVD 88 & Public supply & 125 & na & $125^{\mathrm{a}}$ \\
\hline $\begin{array}{l}\text { Glacial aquifer } \\
\text { system }\end{array}$ & FPS & glacfps 1 & GLACFPS1-01 & WI & Portage & $6 / 11 / 2014$ & 1,100 & NAVD 88 & Observation & 1.3 & 1.2 & 1.3 \\
\hline $\begin{array}{l}\text { Glacial aquifer } \\
\text { system }\end{array}$ & FPS & glacfps1 & GLACFPS1-02 & WI & Portage & $6 / 11 / 2014$ & 1,100 & NAVD 88 & Observation & 1.3 & 1.2 & 1.3 \\
\hline $\begin{array}{l}\text { Glacial aquifer } \\
\text { system }\end{array}$ & FPS & glacfps 1 & GLACFPS1-03 & WI & Portage & $6 / 12 / 2014$ & 1,111 & NAVD 88 & Observation & 1.3 & na & $1.3^{\mathrm{a}}$ \\
\hline $\begin{array}{l}\text { Glacial aquifer } \\
\text { system }\end{array}$ & FPS & glacfps1 & GLACFPS1-04 & WI & Portage & $6 / 12 / 2014$ & 1,100 & NAVD 88 & Observation & 1.3 & 1.2 & 1.3 \\
\hline $\begin{array}{l}\text { Glacial aquifer } \\
\text { system }\end{array}$ & FPS & glacfps 1 & GLACFPS1-05 & WI & Portage & $6 / 12 / 2014$ & 1,100 & NAVD 88 & Observation & 1.3 & 1.2 & 1.3 \\
\hline $\begin{array}{l}\text { Glacial aquifer } \\
\text { system }\end{array}$ & FPS & glacfps1 & GLACFPS1-06 & WI & Portage & $6 / 10 / 2014$ & 1,100 & NAVD 88 & Observation & 1.3 & 1.2 & 1.3 \\
\hline $\begin{array}{l}\text { Glacial aquifer } \\
\text { system }\end{array}$ & FPS & glacfps 1 & GLACFPS1-07 & WI & Portage & $6 / 10 / 2014$ & 1,100 & NAVD 88 & Observation & 1.3 & 1.2 & 1.3 \\
\hline $\begin{array}{l}\text { Glacial aquifer } \\
\text { system }\end{array}$ & FPS & glacfps 1 & GLACFPS1-08 & WI & Portage & $6 / 11 / 2014$ & 1,100 & NAVD 88 & Observation & 1.3 & 1.2 & 1.3 \\
\hline $\begin{array}{l}\text { Glacial aquifer } \\
\text { system }\end{array}$ & FPS & glacfps1 & GLACFPS1-09 & WI & Portage & $6 / 10 / 2014$ & 1,109 & NGVD 29 & Observation & 11.5 & 6.5 & 11.5 \\
\hline $\begin{array}{l}\text { Glacial aquifer } \\
\text { system }\end{array}$ & FPS & glacfps 1 & GLACFPS1-10 & WI & Portage & $6 / 10 / 2014$ & 1,109 & NGVD 29 & Observation & 23.5 & 20.5 & 23.5 \\
\hline $\begin{array}{l}\text { Glacial aquifer } \\
\text { system }\end{array}$ & FPS & glacfps1 & GLACFPS1-11 & WI & Portage & $6 / 11 / 2014$ & 1,109 & NGVD 29 & Observation & 31.5 & 28.5 & 31.5 \\
\hline $\begin{array}{l}\text { Glacial aquifer } \\
\text { system }\end{array}$ & FPS & glacfps1 & GLACFPS1-12 & WI & Portage & $6 / 11 / 2014$ & 1,109 & NGVD 29 & Observation & 44 & 41 & 44 \\
\hline
\end{tabular}


Table 1. Information about wells that have environmental data included in this report.-Continued

[NAWQA, National Water-Quality Assessment; ID, identification; no., number; LS, land surface; ft bls, foot below land surface; lat., latitude, in degrees and minutes; long., longitude in degrees and minutes; PAS, principal aquifer study; FL, Florida; na, not available; NGVD 29, National Geodetic Vertical Datum of 1929; NAVD 88, North American Vertical Datum of 1988; ETN, enhanced trends network; TX, Texas; NM, New Mexico; LUS, land-use study; SC, South Carolina; FPS, flow-path study; MS, Mississippi; VFPS; vertical flow-path study; TN, Tennessee; OK, Oklahoma; AR, Arkansas; CA, California; MO, Missouri; KS, Kansas; MD, Maryland; MSS, modeling support study; DE, Delaware; DC, District of Columbia; MAS, major aquifer study; NV, Nevada; NY, New York; OH, Ohio; MI, Michigan; ID, Idaho; NH, New Hampshire; WI, Wisconsin; OR, Oregon; MN, Minnesota; WA, Washington]

\begin{tabular}{|c|c|c|c|c|c|c|c|c|c|c|c|c|}
\hline \multirow{2}{*}{$\begin{array}{l}\text { Principal and } \\
\text { regional and (or) } \\
\text { other aquifer } \\
\text { information }\end{array}$} & \multirow{2}{*}{$\begin{array}{l}\text { Network } \\
\text { type }\end{array}$} & \multirow{2}{*}{$\begin{array}{l}\text { Network } \\
\text { name }\end{array}$} & \multirow{2}{*}{$\begin{array}{l}\text { NAWOA well } \\
\text { ID no. }\end{array}$} & \multirow{2}{*}{ State } & \multirow{2}{*}{ County } & \multirow{2}{*}{ Sample date } & \multirow{2}{*}{$\begin{array}{l}\text { Altitude } \\
\text { LS }\end{array}$} & \multirow{2}{*}{$\begin{array}{l}\text { Altitude } \\
\text { datum }\end{array}$} & \multirow{2}{*}{ Water use } & \multirow{2}{*}{$\begin{array}{c}\text { Well } \\
\text { depth } \\
\text { (ft bls) }\end{array}$} & \multicolumn{2}{|c|}{$\begin{array}{c}\text { Depth to perforation } \\
\text { (ft bls) }\end{array}$} \\
\hline & & & & & & & & & & & Top & Bottom \\
\hline $\begin{array}{l}\text { Glacial aquifer } \\
\text { system }\end{array}$ & FPS & glacfps1 & GLACFPS1-13 & WI & Portage & $6 / 11 / 2014$ & 1,109 & NGVD 29 & Observation & 61 & 58 & 61 \\
\hline $\begin{array}{l}\text { Glacial aquifer } \\
\text { system }\end{array}$ & FPS & glacfps1 & GLACFPS1-14 & WI & Portage & $6 / 11 / 2014$ & 1,109 & NGVD 29 & Observation & 76 & 73 & 76 \\
\hline $\begin{array}{l}\text { Glacial aquifer } \\
\text { system }\end{array}$ & FPS & glacfps1 & GLACFPS1-15 & WI & Portage & $6 / 10 / 2014$ & 1,135 & NGVD 29 & Observation & 28 & 23 & 28 \\
\hline $\begin{array}{l}\text { Glacial aquifer } \\
\text { system }\end{array}$ & FPS & glacfps1 & GLACFPS1-16 & WI & Portage & $6 / 10 / 2014$ & 1,135 & NGVD 29 & Observation & 53 & 50 & 53 \\
\hline $\begin{array}{l}\text { Glacial aquifer } \\
\text { system }\end{array}$ & FPS & glacfps1 & GLACFPS1-19 & WI & Portage & $6 / 12 / 2014$ & 1,148 & NGVD 29 & Observation & 38 & 35 & 38 \\
\hline $\begin{array}{l}\text { Glacial aquifer } \\
\text { system }\end{array}$ & FPS & glacfps 1 & GLACFPS1-20 & WI & Portage & $6 / 12 / 2014$ & 1,148 & NGVD 29 & Observation & 46 & 43 & 46 \\
\hline $\begin{array}{l}\text { Glacial aquifer } \\
\text { system }\end{array}$ & FPS & glacfps 1 & GLACFPS1-21 & WI & Portage & $6 / 17 / 2014$ & 1,148 & NGVD 29 & Observation & 80 & 55 & 58 \\
\hline $\begin{array}{l}\text { Glacial aquifer } \\
\text { system }\end{array}$ & FPS & glacfps1 & GLACFPS1-22 & WI & Portage & $6 / 18 / 2014$ & 1,148 & NGVD 29 & Observation & 83 & 80 & 83 \\
\hline $\begin{array}{l}\text { Glacial aquifer } \\
\text { system }\end{array}$ & FPS & glacfps1 & GLACFPS1-23 & WI & Portage & $6 / 17 / 2014$ & 1,148 & NGVD 29 & Observation & 99 & 96 & 99 \\
\hline $\begin{array}{l}\text { Glacial aquifer } \\
\text { system }\end{array}$ & FPS & glacfps1 & GLACFPS1-24 & WI & Portage & $6 / 16 / 2014$ & 1,150 & NGVD 29 & Observation & 33 & 23 & 33 \\
\hline $\begin{array}{l}\text { Glacial aquifer } \\
\text { system }\end{array}$ & FPS & glacfps1 & GLACFPS1-25 & WI & Portage & $6 / 17 / 2014$ & 1,150 & NGVD 29 & Observation & 63 & 58 & 63 \\
\hline $\begin{array}{l}\text { Glacial aquifer } \\
\text { system }\end{array}$ & FPS & glacfps1 & GLACFPS1-26 & WI & Portage & $6 / 16 / 2014$ & 1,150 & NGVD 29 & Observation & 93 & 90 & 93 \\
\hline $\begin{array}{l}\text { Glacial aquifer } \\
\text { system }\end{array}$ & FPS & glacfps 1 & GLACFPS1-27 & WI & Portage & $6 / 11 / 2014$ & 1,100 & NAVD 88 & Observation & 1.25 & 1.2 & 1.25 \\
\hline $\begin{array}{l}\text { High Plains } \\
\text { aquifer }\end{array}$ & PAS & hpaqpas1 & HPAQPAS1-64 & $\mathrm{TX}$ & Hockley & $5 / 23 / 2016$ & 3,508 & NAVD 88 & Public supply & 242 & na & $242^{\mathrm{a}}$ \\
\hline $\begin{array}{l}\text { High Plains } \\
\text { aquifer }\end{array}$ & PAS & hpaqpas1 & HPAQPAS1-65 & $\mathrm{TX}$ & Hale & $5 / 24 / 2016$ & 3,402 & NGVD 29 & Public supply & 330 & 240 & 330 \\
\hline
\end{tabular}


Table 1. Information about wells that have environmental data included in this report.-Continued

[NAWQA, National Water-Quality Assessment; ID, identification; no., number; LS, land surface; ft bls, foot below land surface; lat., latitude, in degrees and minutes; long., longitude in degrees and minutes; PAS, principal aquifer study; FL, Florida; na, not available; NGVD 29, National Geodetic Vertical Datum of 1929; NAVD 88, North American Vertical Datum of 1988; ETN, enhanced trends network; TX,

Texas; NM, New Mexico; LUS, land-use study; SC, South Carolina; FPS, flow-path study; MS, Mississippi; VFPS; vertical flow-path study; TN, Tennessee; OK, Oklahoma; AR, Arkansas; CA, California; MO, Missouri; KS, Kansas; MD, Maryland; MSS, modeling support study; DE, Delaware; DC, District of Columbia; MAS, major aquifer study; NV, Nevada; NY, New York; OH, Ohio; MI, Michigan; ID, Idaho;

NH, New Hampshire; WI, Wisconsin; OR, Oregon; MN, Minnesota; WA, Washington]

\begin{tabular}{|c|c|c|c|c|c|c|c|c|c|c|c|c|}
\hline \multirow{2}{*}{$\begin{array}{l}\text { Principal and } \\
\text { regional and (or) } \\
\text { other aquifer } \\
\text { information }\end{array}$} & \multirow{2}{*}{$\begin{array}{l}\text { Network } \\
\text { type }\end{array}$} & \multirow{2}{*}{$\begin{array}{l}\text { Network } \\
\text { name }\end{array}$} & \multirow{2}{*}{$\begin{array}{l}\text { NAWQA well } \\
\text { ID no. }\end{array}$} & \multirow{2}{*}{ State } & \multirow{2}{*}{ County } & \multirow{2}{*}{ Sample date } & \multirow{2}{*}{$\begin{array}{l}\text { Altitude } \\
\text { LS }\end{array}$} & \multirow{2}{*}{$\begin{array}{l}\text { Altitude } \\
\text { datum }\end{array}$} & \multirow{2}{*}{ Water use } & \multirow{2}{*}{$\begin{array}{l}\text { Well } \\
\text { depth } \\
\text { (ft bls) }\end{array}$} & \multicolumn{2}{|c|}{$\begin{array}{c}\text { Depth to perforation } \\
\text { (ft bls) }\end{array}$} \\
\hline & & & & & & & & & & & Top & Bottom \\
\hline $\begin{array}{l}\text { High Plains } \\
\text { aquifer }\end{array}$ & PAS & hpaqpas1 & HPAQPAS1-66 & $\mathrm{TX}$ & Bailey & $5 / 25 / 2016$ & 3,808 & NGVD 29 & Public supply & 201 & 40 & 201 \\
\hline $\begin{array}{l}\text { High Plains } \\
\text { aquifer }\end{array}$ & PAS & hpaqpas1 & HPAQPAS1-67 & $\mathrm{TX}$ & Lubbock & $5 / 26 / 2016$ & 3,186 & NGVD 29 & Public supply & 321 & na & $321^{a}$ \\
\hline $\begin{array}{l}\text { High Plains } \\
\text { aquifer }\end{array}$ & PAS & hpaqpas 1 & HPAQPAS1-68 & $\mathrm{TX}$ & Gaines & $5 / 31 / 2016$ & 3,341 & NAVD 88 & Public supply & 253 & na & $253^{a}$ \\
\hline $\begin{array}{l}\text { High Plains } \\
\text { aquifer }\end{array}$ & PAS & hpaqpas 1 & HPAQPAS1-69 & $\mathrm{TX}$ & Martin & $5 / 31 / 2016$ & 2,909 & NGVD 29 & Public supply & 214 & 127 & 209 \\
\hline $\begin{array}{l}\text { High Plains } \\
\text { aquifer }\end{array}$ & PAS & hpaqpas1 & HPAQPAS1-70 & $\mathrm{TX}$ & Martin & $6 / 1 / 2016$ & 2,683 & NAVD 88 & Public supply & 180 & na & $180^{a}$ \\
\hline $\begin{array}{l}\text { High Plains } \\
\text { aquifer }\end{array}$ & PAS & hpaqpas 1 & HPAQPAS1-71 & $\mathrm{TX}$ & Dawson & $6 / 2 / 2016$ & 3,020 & NAVD 88 & Public supply & 190 & na & $190^{a}$ \\
\hline $\begin{array}{l}\text { High Plains } \\
\text { aquifer }\end{array}$ & PAS & hpaqpas1 & HPAQPAS1-72 & $\mathrm{TX}$ & Lipscomb & 6/13/2016 & 2,624 & NGVD 29 & Public supply & 478 & 270 & 460 \\
\hline $\begin{array}{l}\text { High Plains } \\
\text { aquifer }\end{array}$ & PAS & hpaqpas 1 & HPAQPAS1-73 & $\mathrm{TX}$ & Roberts & $6 / 14 / 2016$ & 3,089 & NGVD 29 & Public supply & 711 & 510 & 695 \\
\hline $\begin{array}{l}\text { High Plains } \\
\text { aquifer }\end{array}$ & PAS & hpaqpas1 & HPAQPAS1-74 & $\mathrm{TX}$ & Wheeler & $6 / 15 / 2016$ & 2,748 & NAVD 88 & Public supply & 262 & na & $262^{a}$ \\
\hline $\begin{array}{l}\text { High Plains } \\
\text { aquifer }\end{array}$ & PAS & hpaqpas 1 & HPAQPAS1-75 & $\mathrm{TX}$ & Carson & $6 / 16 / 2016$ & 3,529 & NGVD 29 & Public supply & 812 & 547 & 812 \\
\hline $\begin{array}{l}\text { High Plains } \\
\text { aquifer }\end{array}$ & PAS & hpaqpas 1 & HPAQPAS1-76 & $\mathrm{TX}$ & Dallam & $6 / 20 / 2016$ & 4,620 & NAVD 88 & Public supply & 363 & na & $363^{a}$ \\
\hline $\begin{array}{l}\text { High Plains } \\
\text { aquifer }\end{array}$ & PAS & hpaqpas 1 & HPAQPAS1-77 & $\mathrm{TX}$ & Parmer & $6 / 21 / 2016$ & 4,017 & NAVD 88 & Public supply & 320 & na & $320^{a}$ \\
\hline $\begin{array}{l}\text { High Plains } \\
\text { aquifer }\end{array}$ & PAS & hpaqpas 1 & HPAQPAS1-78 & $\mathrm{TX}$ & Deaf Smith & $6 / 21 / 2016$ & 3,823 & NGVD 29 & Public supply & 348 & 180 & 340 \\
\hline $\begin{array}{l}\text { High Plains } \\
\text { aquifer }\end{array}$ & PAS & hpaqpas1 & HPAQPAS1-79 & $\mathrm{TX}$ & Moore & $6 / 23 / 2016$ & 3,666 & NAVD 88 & Public supply & 620 & na & $620^{a}$ \\
\hline $\begin{array}{l}\text { High Plains } \\
\text { aquifer }\end{array}$ & PAS & hpaqpas1 & HPAQPAS1-80 & $\mathrm{TX}$ & Sherman & $7 / 13 / 2016$ & 3,695 & NAVD 88 & Public supply & 395 & na & $395^{\mathrm{a}}$ \\
\hline
\end{tabular}


Table 1. Information about wells that have environmental data included in this report.-Continued

[NAWQA, National Water-Quality Assessment; ID, identification; no., number; LS, land surface; ft bls, foot below land surface; lat., latitude, in degrees and minutes; long., longitude in degrees and minutes; PAS, principal aquifer study; FL, Florida; na, not available; NGVD 29, National Geodetic Vertical Datum of 1929; NAVD 88, North American Vertical Datum of 1988; ETN, enhanced trends network; TX, Texas; NM, New Mexico; LUS, land-use study; SC, South Carolina; FPS, flow-path study; MS, Mississippi; VFPS; vertical flow-path study; TN, Tennessee; OK, Oklahoma; AR, Arkansas; CA, California; MO, Missouri; KS, Kansas; MD, Maryland; MSS, modeling support study; DE, Delaware; DC, District of Columbia; MAS, major aquifer study; NV, Nevada; NY, New York; OH, Ohio; MI, Michigan; ID, Idaho; NH, New Hampshire; WI, Wisconsin; OR, Oregon; MN, Minnesota; WA, Washington]

\begin{tabular}{|c|c|c|c|c|c|c|c|c|c|c|c|c|}
\hline \multirow{2}{*}{$\begin{array}{l}\text { Principal and } \\
\text { regional and (or) } \\
\text { other aquifer } \\
\text { information }\end{array}$} & \multirow{2}{*}{$\begin{array}{l}\text { Network } \\
\text { type }\end{array}$} & \multirow{2}{*}{$\begin{array}{l}\text { Network } \\
\text { name }\end{array}$} & \multirow{2}{*}{$\begin{array}{l}\text { NAWOA well } \\
\text { ID no. }\end{array}$} & \multirow{2}{*}{ State } & \multirow{2}{*}{ County } & \multirow{2}{*}{ Sample date } & \multirow{2}{*}{$\begin{array}{c}\text { Altitude } \\
\text { LS }\end{array}$} & \multirow{2}{*}{$\begin{array}{l}\text { Altitude } \\
\text { datum }\end{array}$} & \multirow{2}{*}{ Water use } & \multirow{2}{*}{$\begin{array}{l}\text { Well } \\
\text { depth } \\
\text { (ft bls) }\end{array}$} & \multicolumn{2}{|c|}{$\begin{array}{c}\text { Depth to perforation } \\
\text { (ft bls) }\end{array}$} \\
\hline & & & & & & & & & & & Top & Bottom \\
\hline $\begin{array}{l}\text { Miss. } \\
\text { Embayment- } \\
\text { TX Coastal } \\
\text { Uplands aqui- } \\
\text { fer system }\end{array}$ & FPS & metxfps 1 & METXFPS1-01 & $\mathrm{TN}$ & Hardeman & 9/9/2013 & 500 & NGVD 29 & Observation & 62.7 & 52.2 & 62.2 \\
\hline $\begin{array}{l}\text { Miss. } \\
\text { Embayment- } \\
\text { TX Coastal } \\
\text { Uplands aqui- } \\
\text { fer system }\end{array}$ & FPS & metxfps 1 & METXFPS1-02 & $\mathrm{TN}$ & Fayette & $9 / 11 / 2013$ & 351 & NAVD 88 & Public supply & 120 & na & $120^{a}$ \\
\hline $\begin{array}{l}\text { Miss. } \\
\text { Embayment- } \\
\text { TX Coastal } \\
\text { Uplands aqui- } \\
\text { fer system }\end{array}$ & FPS & metxfps 1 & METXFPS1-03 & $\mathrm{TN}$ & Fayette & $9 / 10 / 2013$ & 385 & NGVD 29 & Public supply & 198 & 162 & 198 \\
\hline $\begin{array}{l}\text { Miss. } \\
\text { Embayment- } \\
\text { TX Coastal } \\
\text { Uplands aqui- } \\
\text { fer system }\end{array}$ & FPS & metxfps1 & METXFPS1-04 & $\mathrm{TN}$ & Fayette & $9 / 10 / 2013$ & 350 & NGVD 29 & Domestic & 210 & 200 & 210 \\
\hline $\begin{array}{l}\text { Miss. } \\
\text { Embayment- } \\
\text { TX Coastal } \\
\text { Uplands aqui- } \\
\text { fer system }\end{array}$ & FPS & metxfps1 & METXFPS1-05 & $\mathrm{TN}$ & Shelby & $9 / 11 / 2013$ & 332 & NGVD 29 & Other & 180 & 160 & 180 \\
\hline $\begin{array}{l}\text { Miss. } \\
\text { Embayment- } \\
\text { TX Coastal } \\
\text { Uplands aqui- } \\
\text { fer system }\end{array}$ & FPS & metxfps 1 & METXFPS1-06 & $\mathrm{TN}$ & Shelby & $8 / 21 / 2013$ & 298 & NGVD 29 & Observation & 300 & 280 & 300 \\
\hline
\end{tabular}


Table 1. Information about wells that have environmental data included in this report.-Continued

[NAWQA, National Water-Quality Assessment; ID, identification; no., number; LS, land surface; ft bls, foot below land surface; lat., latitude, in degrees and minutes; long., longitude in degrees and minutes; PAS, principal aquifer study; FL, Florida; na, not available; NGVD 29, National Geodetic Vertical Datum of 1929; NAVD 88, North American Vertical Datum of 1988; ETN, enhanced trends network; TX,

Texas; NM, New Mexico; LUS, land-use study; SC, South Carolina; FPS, flow-path study; MS, Mississippi; VFPS; vertical flow-path study; TN, Tennessee; OK, Oklahoma; AR, Arkansas; CA, California; MO, Missouri; KS, Kansas; MD, Maryland; MSS, modeling support study; DE, Delaware; DC, District of Columbia; MAS, major aquifer study; NV, Nevada; NY, New York; OH, Ohio; MI, Michigan; ID, Idaho;

NH, New Hampshire; WI, Wisconsin; OR, Oregon; MN, Minnesota; WA, Washington]

\begin{tabular}{|c|c|c|c|c|c|c|c|c|c|c|c|c|}
\hline \multirow{2}{*}{$\begin{array}{l}\text { Principal and } \\
\text { regional and (or) } \\
\text { other aquifer } \\
\text { information }\end{array}$} & \multirow{2}{*}{$\begin{array}{l}\text { Network } \\
\text { type }\end{array}$} & \multirow{2}{*}{$\begin{array}{c}\text { Network } \\
\text { name }\end{array}$} & \multirow{2}{*}{$\begin{array}{l}\text { NAWQA well } \\
\text { ID no. }\end{array}$} & \multirow{2}{*}{ State } & \multirow{2}{*}{ County } & \multirow{2}{*}{ Sample date } & \multirow{2}{*}{$\begin{array}{c}\text { Altitude } \\
\text { LS }\end{array}$} & \multirow{2}{*}{$\begin{array}{l}\text { Altitude } \\
\text { datum }\end{array}$} & \multirow{2}{*}{ Water use } & \multirow{2}{*}{$\begin{array}{l}\text { Well } \\
\text { depth } \\
\text { (ft bls) }\end{array}$} & \multicolumn{2}{|c|}{$\begin{array}{c}\text { Depth to perforation } \\
\text { (ft bls) }\end{array}$} \\
\hline & & & & & & & & & & & Top & Bottom \\
\hline $\begin{array}{l}\text { Miss. } \\
\text { Embayment- } \\
\text { TX Coastal } \\
\text { Uplands aqui- } \\
\text { fer system }\end{array}$ & FPS & metxfps 1 & METXFPS1-07 & $\mathrm{TN}$ & Shelby & $8 / 29 / 2013$ & 316 & NGVD 29 & Public supply & 582 & 490 & 578 \\
\hline $\begin{array}{l}\text { Miss. } \\
\text { Embayment- } \\
\text { TX Coastal } \\
\text { Uplands aqui- } \\
\text { fer system }\end{array}$ & FPS & metxfps 1 & METXFPS1-09 & MS & Benton & $8 / 15 / 2013$ & 411 & NAVD 88 & Observation & 23 & 13 & 23 \\
\hline $\begin{array}{l}\text { Miss. } \\
\text { Embayment- } \\
\text { TX Coastal } \\
\text { Uplands aqui- } \\
\text { fer system }\end{array}$ & FPS & metxfps1 & METXFPS1-10 & $\mathrm{TN}$ & Fayette & $8 / 14 / 2013$ & 421 & NGVD 29 & Domestic & 95 & 85 & 95 \\
\hline $\begin{array}{l}\text { Miss. } \\
\text { Embayment- } \\
\text { TX Coastal } \\
\text { Uplands aqui- } \\
\text { fer system }\end{array}$ & FPS & metxfps 1 & METXFPS1-11 & $\mathrm{TN}$ & Fayette & $8 / 22 / 2013$ & 432 & NGVD 29 & Domestic & 200 & 180 & 200 \\
\hline $\begin{array}{l}\text { Miss. } \\
\text { Embayment- } \\
\text { TX Coastal } \\
\text { Uplands aqui- } \\
\text { fer system }\end{array}$ & FPS & metxfps 1 & METXFPS1-12 & $\mathrm{TN}$ & Fayette & $8 / 20 / 2013$ & 311 & NGVD 29 & Public supply & 174 & 130 & 174 \\
\hline $\begin{array}{l}\text { Miss. } \\
\text { Embayment- } \\
\text { TX Coastal } \\
\text { Uplands aqui- } \\
\text { fer system }\end{array}$ & FPS & metxfps1 & METXFPS1-13 & $\mathrm{TN}$ & Shelby & $8 / 14 / 2013$ & 383 & NGVD 29 & Public supply & 263 & 223 & 263 \\
\hline
\end{tabular}


Table 1. Information about wells that have environmental data included in this report.-Continued

[NAWQA, National Water-Quality Assessment; ID, identification; no., number; LS, land surface; $\mathrm{ft}$ bls, foot below land surface; lat., latitude, in degrees and minutes; long., longitude in degrees and minutes; PAS, principal aquifer study; FL, Florida; na, not available; NGVD 29, National Geodetic Vertical Datum of 1929; NAVD 88, North American Vertical Datum of 1988; ETN, enhanced trends network; TX, Texas; NM, New Mexico; LUS, land-use study; SC, South Carolina; FPS, flow-path study; MS, Mississippi; VFPS; vertical flow-path study; TN, Tennessee; OK, Oklahoma; AR, Arkansas; CA, California; MO, Missouri; KS, Kansas; MD, Maryland; MSS, modeling support study; DE, Delaware; DC, District of Columbia; MAS, major aquifer study; NV, Nevada; NY, New York; OH, Ohio; MI, Michigan; ID, Idaho; NH, New Hampshire; WI, Wisconsin; OR, Oregon; MN, Minnesota; WA, Washington]

\begin{tabular}{|c|c|c|c|c|c|c|c|c|c|c|c|c|}
\hline \multirow{2}{*}{$\begin{array}{l}\text { Principal and } \\
\text { regional and (or) } \\
\text { other aquifer } \\
\text { information }\end{array}$} & \multirow{2}{*}{$\begin{array}{l}\text { Network } \\
\text { type }\end{array}$} & \multirow{2}{*}{$\begin{array}{l}\text { Network } \\
\text { name }\end{array}$} & \multirow{2}{*}{$\begin{array}{l}\text { NAWQA well } \\
\text { ID no. }\end{array}$} & \multirow{2}{*}{ State } & \multirow{2}{*}{ County } & \multirow{2}{*}{ Sample date } & \multirow{2}{*}{$\begin{array}{l}\text { Altitude } \\
\text { LS }\end{array}$} & \multirow{2}{*}{$\begin{array}{l}\text { Altitude } \\
\text { datum }\end{array}$} & \multirow{2}{*}{ Water use } & \multirow{2}{*}{$\begin{array}{c}\text { Well } \\
\text { depth } \\
\text { (ft bls) }\end{array}$} & \multicolumn{2}{|c|}{$\begin{array}{c}\text { Depth to perforation } \\
\text { (ft bls) }\end{array}$} \\
\hline & & & & & & & & & & & Top & Bottom \\
\hline $\begin{array}{l}\text { Miss. } \\
\text { Embayment- } \\
\text { TX Coastal } \\
\text { Uplands aqui- } \\
\text { fer system }\end{array}$ & FPS & metxfps 1 & METXFPS1-14 & $\mathrm{TN}$ & Shelby & $8 / 20 / 2013$ & 352 & NGVD 29 & Public supply & 269 & 190 & 265 \\
\hline $\begin{array}{l}\text { Miss. } \\
\text { Embayment- } \\
\text { TX Coastal } \\
\text { Uplands aqui- } \\
\text { fer system }\end{array}$ & FPS & metxfps1 & METXFPS1-15 & $\mathrm{TN}$ & Shelby & $8 / 21 / 2013$ & 360 & NGVD 29 & Public supply & 304 & 240 & 300 \\
\hline $\begin{array}{l}\text { Miss. } \\
\text { Embayment- } \\
\text { TX Coastal } \\
\text { Uplands aqui- } \\
\text { fer system }\end{array}$ & FPS & metxfps 1 & METXFPS1-16 & $\mathrm{TN}$ & Shelby & $8 / 27 / 2013$ & 280 & NGVD 29 & Public supply & 598 & 490 & 590 \\
\hline $\begin{array}{l}\text { Miss. } \\
\text { Embayment- } \\
\text { TX Coastal } \\
\text { Uplands aqui- } \\
\text { fer system }\end{array}$ & FPS & metxfps 1 & METXFPS1-18 & $\mathrm{TN}$ & Shelby & $8 / 19 / 2013$ & 290 & NGVD 29 & Observation & 87 & 77 & 87 \\
\hline $\begin{array}{l}\text { Miss. } \\
\text { Embayment- } \\
\text { TX Coastal } \\
\text { Uplands aqui- } \\
\text { fer system }\end{array}$ & FPS & metxfps 1 & METXFPS1-19 & $\mathrm{TN}$ & Shelby & $9 / 12 / 2013$ & 360 & NGVD 29 & Other & 155 & 135 & 155 \\
\hline $\begin{array}{l}\text { Miss. } \\
\text { Embayment- } \\
\text { TX Coastal } \\
\text { Uplands aqui- } \\
\text { fer system }\end{array}$ & FPS & metxfps 1 & METXFPS1-20 & $\mathrm{TN}$ & Shelby & $8 / 13 / 2013$ & 300 & NGVD 29 & Observation & 107.7 & 97.7 & 107.7 \\
\hline
\end{tabular}


Table 1. Information about wells that have environmental data included in this report.-Continued

[NAWQA, National Water-Quality Assessment; ID, identification; no., number; LS, land surface; ft bls, foot below land surface; lat., latitude, in degrees and minutes; long., longitude in degrees and minutes; PAS, principal aquifer study; FL, Florida; na, not available; NGVD 29, National Geodetic Vertical Datum of 1929; NAVD 88, North American Vertical Datum of 1988; ETN, enhanced trends network; TX,

Texas; NM, New Mexico; LUS, land-use study; SC, South Carolina; FPS, flow-path study; MS, Mississippi; VFPS; vertical flow-path study; TN, Tennessee; OK, Oklahoma; AR, Arkansas; CA, California; MO, Missouri; KS, Kansas; MD, Maryland; MSS, modeling support study; DE, Delaware; DC, District of Columbia; MAS, major aquifer study; NV, Nevada; NY, New York; OH, Ohio; MI, Michigan; ID, Idaho;

NH, New Hampshire; WI, Wisconsin; OR, Oregon; MN, Minnesota; WA, Washington]

\begin{tabular}{|c|c|c|c|c|c|c|c|c|c|c|c|c|}
\hline \multirow{2}{*}{$\begin{array}{l}\text { Principal and } \\
\text { regional and (or) } \\
\text { other aquifer } \\
\text { information }\end{array}$} & \multirow{2}{*}{$\begin{array}{l}\text { Network } \\
\text { type }\end{array}$} & \multirow{2}{*}{$\begin{array}{l}\text { Network } \\
\text { name }\end{array}$} & \multirow{2}{*}{$\begin{array}{l}\text { NAWQA well } \\
\text { ID no. }\end{array}$} & \multirow{2}{*}{ State } & \multirow{2}{*}{ County } & \multirow{2}{*}{ Sample date } & \multirow{2}{*}{$\begin{array}{c}\text { Altitude } \\
\text { LS }\end{array}$} & \multirow{2}{*}{$\begin{array}{l}\text { Altitude } \\
\text { datum }\end{array}$} & \multirow{2}{*}{ Water use } & \multirow{2}{*}{$\begin{array}{l}\text { Well } \\
\text { depth } \\
\text { (ft bls) }\end{array}$} & \multicolumn{2}{|c|}{$\begin{array}{c}\text { Depth to perforation } \\
\text { (ft bls) }\end{array}$} \\
\hline & & & & & & & & & & & Top & Bottom \\
\hline $\begin{array}{l}\text { Miss. } \\
\text { Embayment- } \\
\text { TX Coastal } \\
\text { Uplands aqui- } \\
\text { fer system }\end{array}$ & FPS & metxfps 1 & METXFPS1-21 & $\mathrm{TN}$ & Shelby & $8 / 12 / 2013$ & 268 & NGVD 29 & Observation & 76.1 & 66.1 & 76.1 \\
\hline $\begin{array}{l}\text { Miss. } \\
\text { Embayment- } \\
\text { TX Coastal } \\
\text { Uplands aqui- } \\
\text { fer system }\end{array}$ & FPS & metxfps 1 & METXFPS1-22 & $\mathrm{TN}$ & Shelby & $8 / 27 / 2013$ & 259 & NGVD 29 & Observation & 91 & 81 & 91 \\
\hline $\begin{array}{l}\text { Miss. } \\
\text { Embayment- } \\
\text { TX Coastal } \\
\text { Uplands aqui- } \\
\text { fer system }\end{array}$ & $\begin{array}{l}\text { ETN/ } \\
\text { FPS }\end{array}$ & $\begin{array}{l}\text { metxetn1/ } \\
\text { metxfps1 }\end{array}$ & $\begin{array}{l}\text { METXETN1-01/ } \\
\text { METXFPS1-08 }\end{array}$ & $\mathrm{TN}$ & Shelby & $\begin{array}{c}8 / 28 / 2013 ; \\
4 / 26,6 / 22 \\
8 / 22,10 / 31 \\
12 / 13 / 2016\end{array}$ & 320 & NGVD 29 & Public supply & 624 & 520 & 624 \\
\hline $\begin{array}{l}\text { Miss. } \\
\text { Embayment- } \\
\text { TX Coastal } \\
\text { Uplands aqui- } \\
\text { fer system }\end{array}$ & $\begin{array}{l}\text { ETN/ } \\
\text { FPS }\end{array}$ & $\begin{array}{l}\text { metxetn1/ } \\
\text { metxfps } 1\end{array}$ & $\begin{array}{l}\text { METXETN1-02/ } \\
\text { METXFPS1-17 }\end{array}$ & $\mathrm{TN}$ & Shelby & $\begin{array}{l}8 / 28,11 / 19 \\
12 / 16 / 2013 \\
2 / 3 / 2014 \\
2 / 29,4 / 26 \\
6 / 22,8 / 23 \\
10 / 31 \\
12 / 13 / 2016\end{array}$ & 310 & NGVD 29 & Observation & 90 & 80 & 90 \\
\hline $\begin{array}{l}\text { Northern Atlantic } \\
\text { Coastal Plain } \\
\text { aquifer system }\end{array}$ & ETN & nacpetn1 & NACPETN1-01 & $\mathrm{DE}$ & Sussex & $\begin{array}{c}3 / 2,3 / 23,4 / 26 \\
6 / 28,8 / 10 \\
10 / 4 / 2016\end{array}$ & 16 & NGVD 29 & Observation & 22 & 19 & 22 \\
\hline $\begin{array}{l}\text { Northern Atlantic } \\
\text { Coastal Plain } \\
\text { aquifer system }\end{array}$ & ETN & nacpetn1 & NACPETN1-02 & $\mathrm{DE}$ & Sussex & $\begin{array}{c}3 / 2,4 / 26 \\
8 / 9,8 / 29 \\
10 / 5 / 2016\end{array}$ & 13.8 & NGVD 29 & Public supply & 135 & 85 & 135 \\
\hline $\begin{array}{l}\text { Northern Atlantic } \\
\text { Coastal Plain } \\
\text { aquifer system }\end{array}$ & ETN & nacpetn1 & NACPETN1-03 & $\mathrm{DE}$ & Sussex & $\begin{array}{c}3 / 3,4 / 27,5 / 6 \\
6 / 29,8 / 11 \\
10 / 4 / 2016\end{array}$ & 41.6 & NGVD 29 & Public supply & 119 & 100 & 119 \\
\hline
\end{tabular}


Table 1. Information about wells that have environmental data included in this report.-Continued

[NAWQA, National Water-Quality Assessment; ID, identification; no., number; LS, land surface; ft bls, foot below land surface; lat., latitude, in degrees and minutes; long., longitude in degrees and minutes; PAS, principal aquifer study; FL, Florida; na, not available; NGVD 29, National Geodetic Vertical Datum of 1929; NAVD 88, North American Vertical Datum of 1988; ETN, enhanced trends network; TX, Texas; NM, New Mexico; LUS, land-use study; SC, South Carolina; FPS, flow-path study; MS, Mississippi; VFPS; vertical flow-path study; TN, Tennessee; OK, Oklahoma; AR, Arkansas; CA, California; MO, Missouri; KS, Kansas; MD, Maryland; MSS, modeling support study; DE, Delaware; DC, District of Columbia; MAS, major aquifer study; NV, Nevada; NY, New York; OH, Ohio; MI, Michigan; ID, Idaho; NH, New Hampshire; WI, Wisconsin; OR, Oregon; MN, Minnesota; WA, Washington]

\begin{tabular}{|c|c|c|c|c|c|c|c|c|c|c|c|c|}
\hline \multirow{2}{*}{$\begin{array}{l}\text { Principal and } \\
\text { regional and (or) } \\
\text { other aquifer } \\
\text { information }\end{array}$} & \multirow{2}{*}{$\begin{array}{l}\text { Network } \\
\text { type }\end{array}$} & \multirow{2}{*}{$\begin{array}{l}\text { Network } \\
\text { name }\end{array}$} & \multirow{2}{*}{$\begin{array}{l}\text { NAWQA well } \\
\text { ID no. }\end{array}$} & \multirow{2}{*}{ State } & \multirow{2}{*}{ County } & \multirow{2}{*}{ Sample date } & \multirow{2}{*}{$\begin{array}{l}\text { Altitude } \\
\text { LS }\end{array}$} & \multirow{2}{*}{$\begin{array}{l}\text { Altitude } \\
\text { datum }\end{array}$} & \multirow{2}{*}{ Water use } & \multirow{2}{*}{$\begin{array}{l}\text { Well } \\
\text { depth } \\
\text { (ft bls) }\end{array}$} & \multicolumn{2}{|c|}{$\begin{array}{c}\text { Depth to perforation } \\
\text { (ft bls) }\end{array}$} \\
\hline & & & & & & & & & & & Top & Bottom \\
\hline $\begin{array}{l}\text { Northern Atlantic } \\
\text { Coastal Plain } \\
\text { aquifer system }\end{array}$ & MSS & nacpmss 1 & NACPMSS1-01 & $\mathrm{DC}$ & $\begin{array}{l}\text { District of } \\
\text { Columbia }\end{array}$ & $12 / 19 / 2016$ & 60.6 & NAVD 88 & Observation & 290 & 140 & 290 \\
\hline $\begin{array}{l}\text { Northern Atlantic } \\
\text { Coastal Plain } \\
\text { aquifer system }\end{array}$ & MSS & nacpmss1 & NACPMSS1-02 & DC & $\begin{array}{l}\text { District of } \\
\text { Columbia }\end{array}$ & $12 / 6 / 2016$ & 58.8 & NAVD 88 & Observation & 265 & 255 & 265 \\
\hline $\begin{array}{l}\text { Northern Atlantic } \\
\text { Coastal Plain } \\
\text { aquifer system }\end{array}$ & MSS & nacpmss1 & NACPMSS1-03 & $\mathrm{DC}$ & $\begin{array}{l}\text { District of } \\
\text { Columbia }\end{array}$ & $12 / 20 / 2016$ & 142.6 & NAVD 88 & Observation & 60 & 49.5 & 59.5 \\
\hline $\begin{array}{l}\text { Northern Atlantic } \\
\text { Coastal Plain } \\
\text { aquifer system }\end{array}$ & MSS & nacpmss 1 & NACPMSS1-04 & MD & $\begin{array}{l}\text { Prince } \\
\text { George's }\end{array}$ & $8 / 22 / 2016$ & 180 & NAVD 88 & Other & 345 & 290 & 340 \\
\hline $\begin{array}{l}\text { Northern Atlantic } \\
\text { Coastal Plain } \\
\text { aquifer system }\end{array}$ & MSS & nacpmss 1 & NACPMSS1-05 & MD & $\begin{array}{l}\text { Prince } \\
\text { George's }\end{array}$ & $8 / 23 / 2016$ & 185 & NGVD 29 & Other & 430 & 385 & 425 \\
\hline $\begin{array}{l}\text { Northern Atlantic } \\
\text { Coastal Plain } \\
\text { aquifer system }\end{array}$ & MSS & nacpmss 1 & NACPMSS1-06 & MD & $\begin{array}{l}\text { Anne } \\
\text { Arundel }\end{array}$ & $8 / 24 / 2016$ & 126 & NGVD 29 & Public supply & 295 & 275 & 295 \\
\hline $\begin{array}{l}\text { Northern Atlantic } \\
\text { Coastal Plain } \\
\text { aquifer system }\end{array}$ & MSS & nacpmss1 & NACPMSS1-07 & MD & $\begin{array}{l}\text { Prince } \\
\text { George's }\end{array}$ & $10 / 26 / 2016$ & 212 & NAVD 88 & unknown & 607 & 533 & 607 \\
\hline $\begin{array}{l}\text { Northern Atlantic } \\
\text { Coastal Plain } \\
\text { aquifer system }\end{array}$ & MSS & nacpmss1 & NACPMSS1-08 & MD & Charles & $10 / 27 / 2016$ & 204.6 & NGVD 29 & Other & 397 & 377 & 397 \\
\hline $\begin{array}{l}\text { Northern Atlantic } \\
\text { Coastal Plain } \\
\text { aquifer system }\end{array}$ & MSS & nacpmss 1 & NACPMSS1-09 & $\mathrm{MD}$ & $\begin{array}{l}\text { Anne } \\
\text { Arundel }\end{array}$ & $11 / 15 / 2016$ & 70 & NGVD 29 & Other & 280 & 259 & 277 \\
\hline $\begin{array}{l}\text { Northern Atlantic } \\
\text { Coastal Plain } \\
\text { aquifer system }\end{array}$ & MSS & nacpmss 1 & NACPMSS1-10 & MD & $\begin{array}{l}\text { Prince } \\
\text { George's }\end{array}$ & $11 / 16 / 2016$ & 240.9 & NGVD 29 & Other & 342 & 317 & 342 \\
\hline
\end{tabular}


Table 1. Information about wells that have environmental data included in this report.-Continued

[NAWQA, National Water-Quality Assessment; ID, identification; no., number; LS, land surface; ft bls, foot below land surface; lat., latitude, in degrees and minutes; long., longitude in degrees and minutes; PAS, principal aquifer study; FL, Florida; na, not available; NGVD 29, National Geodetic Vertical Datum of 1929; NAVD 88, North American Vertical Datum of 1988; ETN, enhanced trends network; TX,

Texas; NM, New Mexico; LUS, land-use study; SC, South Carolina; FPS, flow-path study; MS, Mississippi; VFPS; vertical flow-path study; TN, Tennessee; OK, Oklahoma; AR, Arkansas; CA, California; MO, Missouri; KS, Kansas; MD, Maryland; MSS, modeling support study; DE, Delaware; DC, District of Columbia; MAS, major aquifer study; NV, Nevada; NY, New York; OH, Ohio; MI, Michigan; ID, Idaho;

NH, New Hampshire; WI, Wisconsin; OR, Oregon; MN, Minnesota; WA, Washington]

\begin{tabular}{|c|c|c|c|c|c|c|c|c|c|c|c|c|}
\hline \multirow{2}{*}{$\begin{array}{l}\text { Principal and } \\
\text { regional and (or) } \\
\text { other aquifer } \\
\text { information }\end{array}$} & \multirow{2}{*}{$\begin{array}{l}\text { Network } \\
\text { type }\end{array}$} & \multirow{2}{*}{$\begin{array}{l}\text { Network } \\
\text { name }\end{array}$} & \multirow{2}{*}{$\begin{array}{l}\text { NAWOA well } \\
\text { ID no. }\end{array}$} & \multirow{2}{*}{ State } & \multirow{2}{*}{ County } & \multirow{2}{*}{ Sample date } & \multirow{2}{*}{$\begin{array}{c}\text { Altitude } \\
\text { LS }\end{array}$} & \multirow{2}{*}{$\begin{array}{l}\text { Altitude } \\
\text { datum }\end{array}$} & \multirow{2}{*}{ Water use } & \multirow{2}{*}{$\begin{array}{c}\text { Well } \\
\text { depth } \\
\text { (ft bls) }\end{array}$} & \multicolumn{2}{|c|}{$\begin{array}{c}\text { Depth to perforation } \\
\text { (ft bls) }\end{array}$} \\
\hline & & & & & & & & & & & Top & Bottom \\
\hline $\begin{array}{l}\text { Northern Atlantic } \\
\text { Coastal Plain } \\
\text { aquifer system }\end{array}$ & MSS & nacpmss1 & NACPMSS1-11 & MD & $\begin{array}{l}\text { Prince } \\
\text { George's }\end{array}$ & $11 / 17 / 2016$ & 240 & NGVD 29 & Other & 645 & 530 & 640 \\
\hline $\begin{array}{l}\text { Northern Atlantic } \\
\text { Coastal Plain } \\
\text { aquifer system }\end{array}$ & MSS & nacpmss 1 & NACPMSS1-12 & $\mathrm{MD}$ & $\begin{array}{l}\text { Anne } \\
\text { Arundel }\end{array}$ & $11 / 21 / 2016$ & 162.8 & NAVD 88 & Observation & 240 & 215 & 235 \\
\hline $\begin{array}{l}\text { Northern Atlantic } \\
\text { Coastal Plain } \\
\text { aquifer system }\end{array}$ & MSS & nacpmss1 & NACPMSS1-13 & $\mathrm{MD}$ & $\begin{array}{l}\text { Prince } \\
\quad \text { George's }\end{array}$ & $11 / 21 / 2016$ & 28 & NAVD 88 & unknown & 415 & 385 & 391 \\
\hline $\begin{array}{l}\text { Northern Atlantic } \\
\text { Coastal Plain } \\
\text { aquifer system }\end{array}$ & MSS & nacpmss 1 & NACPMSS1-14 & MD & $\begin{array}{l}\text { Anne } \\
\text { Arundel }\end{array}$ & $11 / 22 / 2016$ & 163.4 & NAVD 88 & Observation & 125 & 100 & 120 \\
\hline $\begin{array}{l}\text { Northern Atlantic } \\
\text { Coastal Plain } \\
\text { aquifer system }\end{array}$ & MSS & nacpmss1 & NACPMSS1-15 & MD & $\begin{array}{l}\text { Anne } \\
\text { Arundel }\end{array}$ & $11 / 22 / 2016$ & 51 & NAVD 88 & Other & 200 & 186 & 193 \\
\hline $\begin{array}{l}\text { Northern Atlantic } \\
\text { Coastal Plain } \\
\text { aquifer system }\end{array}$ & MSS & nacpmss 1 & NACPMSS1-16 & MD & $\begin{array}{l}\text { Anne } \\
\text { Arundel }\end{array}$ & $11 / 30 / 2016$ & 35 & NAVD 88 & Other & 360 & 349 & 356 \\
\hline $\begin{array}{l}\text { Northern Atlantic } \\
\text { Coastal Plain } \\
\text { aquifer system }\end{array}$ & MSS & nacpmss1 & NACPMSS1-17 & MD & Calvert & $12 / 12 / 2016$ & 137.9 & NGVD 29 & Domestic & 320 & 310 & 320 \\
\hline $\begin{array}{l}\text { Northern Atlantic } \\
\text { Coastal Plain } \\
\text { aquifer system }\end{array}$ & MSS & nacpmss1 & NACPMSS1-18 & MD & Calvert & $12 / 13 / 2016$ & 138.8 & NGVD 29 & Observation & 170 & 160 & 170 \\
\hline $\begin{array}{l}\text { Northern Atlantic } \\
\text { Coastal Plain } \\
\text { aquifer system }\end{array}$ & MSS & nacpmss1 & NACPMSS1-19 & MD & $\begin{array}{l}\text { Prince } \\
\text { George's }\end{array}$ & $12 / 20 / 2016$ & 95.8 & NGVD 29 & Observation & 155 & 150 & 155 \\
\hline $\begin{array}{l}\text { Northern Atlantic } \\
\text { Coastal Plain } \\
\text { aquifer system }\end{array}$ & MSS & nacpmss1 & NACPMSS1-20 & MD & $\begin{array}{l}\text { Anne } \\
\text { Arundel }\end{array}$ & $12 / 28 / 2016$ & 51.3 & NGVD 29 & Observation & 177 & 142 & 172 \\
\hline
\end{tabular}


Table 1. Information about wells that have environmental data included in this report.-Continued

[NAWQA, National Water-Quality Assessment; ID, identification; no., number; LS, land surface; ft bls, foot below land surface; lat., latitude, in degrees and minutes; long., longitude in degrees and minutes; PAS, principal aquifer study; FL, Florida; na, not available; NGVD 29, National Geodetic Vertical Datum of 1929; NAVD 88, North American Vertical Datum of 1988; ETN, enhanced trends network; TX, Texas; NM, New Mexico; LUS, land-use study; SC, South Carolina; FPS, flow-path study; MS, Mississippi; VFPS; vertical flow-path study; TN, Tennessee; OK, Oklahoma; AR, Arkansas; CA, California; MO, Missouri; KS, Kansas; MD, Maryland; MSS, modeling support study; DE, Delaware; DC, District of Columbia; MAS, major aquifer study; NV, Nevada; NY, New York; OH, Ohio; MI, Michigan; ID, Idaho; NH, New Hampshire; WI, Wisconsin; OR, Oregon; MN, Minnesota; WA, Washington]

\begin{tabular}{|c|c|c|c|c|c|c|c|c|c|c|c|c|}
\hline \multirow{2}{*}{$\begin{array}{l}\text { Principal and } \\
\text { regional and (or) } \\
\text { other aquifer } \\
\text { information }\end{array}$} & \multirow{2}{*}{$\begin{array}{l}\text { Network } \\
\text { type }\end{array}$} & \multirow{2}{*}{$\begin{array}{l}\text { Network } \\
\text { name }\end{array}$} & \multirow{2}{*}{$\begin{array}{l}\text { NAWOA well } \\
\text { ID no. }\end{array}$} & \multirow{2}{*}{ State } & \multirow{2}{*}{ County } & \multirow{2}{*}{ Sample date } & \multirow{2}{*}{$\begin{array}{l}\text { Altitude } \\
\text { LS }\end{array}$} & \multirow{2}{*}{$\begin{array}{l}\text { Altitude } \\
\text { datum }\end{array}$} & \multirow{2}{*}{ Water use } & \multirow{2}{*}{$\begin{array}{l}\text { Well } \\
\text { depth } \\
\text { (ft bls) }\end{array}$} & \multicolumn{2}{|c|}{$\begin{array}{c}\text { Depth to perforation } \\
\text { (ft bls) }\end{array}$} \\
\hline & & & & & & & & & & & Top & Bottom \\
\hline $\begin{array}{l}\text { Northern Atlantic } \\
\text { Coastal Plain } \\
\text { aquifer system }\end{array}$ & MSS & nacpmss 2 & NACPMSS2-01 & NY & Suffolk & $8 / 16 / 2016$ & 75 & NGVD 29 & Public supply & 388 & 307 & 367 \\
\hline $\begin{array}{l}\text { Northern Atlantic } \\
\text { Coastal Plain } \\
\text { aquifer system }\end{array}$ & MSS & nacpmss 2 & NACPMSS2-02 & NY & Suffolk & $8 / 17 / 2016$ & 71 & NGVD 29 & Public supply & 543 & 425.6 & 529 \\
\hline $\begin{array}{l}\text { Northern Atlantic } \\
\text { Coastal Plain } \\
\text { aquifer system }\end{array}$ & MSS & nacpmss 2 & NACPMSS2-03 & NY & Suffolk & $8 / 17 / 2016$ & 108 & NAVD 88 & Domestic & 734 & 670 & 730.8 \\
\hline $\begin{array}{l}\text { Northern Atlantic } \\
\text { Coastal Plain } \\
\text { aquifer system }\end{array}$ & MSS & nacpmss2 & NACPMSS2-04 & NY & Nassau & $8 / 22 / 2016$ & 145 & NGVD 29 & Public supply & 283 & 221 & 280 \\
\hline $\begin{array}{l}\text { Northern Atlantic } \\
\text { Coastal Plain } \\
\text { aquifer system }\end{array}$ & MSS & nacpmss2 & NACPMSS2-05 & NY & Suffolk & $8 / 23 / 2016$ & 185 & NGVD 29 & Public supply & 488 & 423 & 483 \\
\hline $\begin{array}{l}\text { Northern Atlantic } \\
\text { Coastal Plain } \\
\text { aquifer system }\end{array}$ & MSS & nacpmss 2 & NACPMSS2-06 & NY & Suffolk & $8 / 23 / 2016$ & 157 & NAVD 88 & Domestic & 468 & 410 & 465 \\
\hline $\begin{array}{l}\text { Northern Atlantic } \\
\text { Coastal Plain } \\
\text { aquifer system }\end{array}$ & MSS & nacpmss2 & NACPMSS2-07 & NY & Suffolk & $8 / 24 / 2016$ & 110 & NGVD 29 & Public supply & 900 & 585 & 645 \\
\hline $\begin{array}{l}\text { Northern Atlantic } \\
\text { Coastal Plain } \\
\text { aquifer system }\end{array}$ & MSS & nacpmss 2 & NACPMSS2-08 & NY & Suffolk & $8 / 24 / 2016$ & 120 & NGVD 29 & Public supply & 312 & 260 & 310 \\
\hline $\begin{array}{l}\text { Northern Atlantic } \\
\text { Coastal Plain } \\
\text { aquifer system }\end{array}$ & MSS & nacpmss2 & NACPMSS2-09 & NY & Suffolk & $8 / 25 / 2016$ & 39 & NAVD 88 & Domestic & 620 & 561 & 616 \\
\hline $\begin{array}{l}\text { Northern Atlantic } \\
\text { Coastal Plain } \\
\text { aquifer system }\end{array}$ & MSS & nacpmss 2 & NACPMSS2-10 & NY & Nassau & $8 / 30 / 2016$ & 26 & NGVD 29 & Public supply & 626 & 566 & 626 \\
\hline
\end{tabular}


Table 1. Information about wells that have environmental data included in this report.-Continued

[NAWQA, National Water-Quality Assessment; ID, identification; no., number; LS, land surface; ft bls, foot below land surface; lat., latitude, in degrees and minutes; long., longitude in degrees and minutes; PAS, principal aquifer study; FL, Florida; na, not available; NGVD 29, National Geodetic Vertical Datum of 1929; NAVD 88, North American Vertical Datum of 1988; ETN, enhanced trends network; TX,

Texas; NM, New Mexico; LUS, land-use study; SC, South Carolina; FPS, flow-path study; MS, Mississippi; VFPS; vertical flow-path study; TN, Tennessee; OK, Oklahoma; AR, Arkansas; CA, California; MO, Missouri; KS, Kansas; MD, Maryland; MSS, modeling support study; DE, Delaware; DC, District of Columbia; MAS, major aquifer study; NV, Nevada; NY, New York; OH, Ohio; MI, Michigan; ID, Idaho;

NH, New Hampshire; WI, Wisconsin; OR, Oregon; MN, Minnesota; WA, Washington]

\begin{tabular}{|c|c|c|c|c|c|c|c|c|c|c|c|c|}
\hline \multirow{2}{*}{$\begin{array}{l}\text { Principal and } \\
\text { regional and (or) } \\
\text { other aquifer } \\
\text { information }\end{array}$} & \multirow{2}{*}{$\begin{array}{l}\text { Network } \\
\text { type }\end{array}$} & \multirow{2}{*}{$\begin{array}{l}\text { Network } \\
\text { name }\end{array}$} & \multirow{2}{*}{$\begin{array}{l}\text { NAWOA well } \\
\text { ID no. }\end{array}$} & \multirow{2}{*}{ State } & \multirow{2}{*}{ County } & \multirow{2}{*}{ Sample date } & \multirow{2}{*}{$\begin{array}{l}\text { Altitude } \\
\text { LS }\end{array}$} & \multirow{2}{*}{$\begin{array}{l}\text { Altitude } \\
\text { datum }\end{array}$} & \multirow{2}{*}{ Water use } & \multirow{2}{*}{$\begin{array}{l}\text { Well } \\
\text { depth } \\
\text { (ft bls) }\end{array}$} & \multicolumn{2}{|c|}{$\begin{array}{c}\text { Depth to perforation } \\
\text { (ft bls) }\end{array}$} \\
\hline & & & & & & & & & & & Top & Bottom \\
\hline $\begin{array}{l}\text { Northern Atlantic } \\
\text { Coastal Plain } \\
\text { aquifer system }\end{array}$ & MSS & nacpmss2 & NACPMSS2-11 & NY & Nassau & $8 / 31 / 2016$ & 61 & NGVD 29 & Public supply & 449 & 394 & 444 \\
\hline $\begin{array}{l}\text { Northern Atlantic } \\
\text { Coastal Plain } \\
\text { aquifer system }\end{array}$ & MSS & nacpmss2 & NACPMSS2-12 & NY & Nassau & $8 / 31 / 2016$ & 101 & NGVD 29 & Public supply & 227 & 175 & 222 \\
\hline $\begin{array}{l}\text { Northern Atlantic } \\
\text { Coastal Plain } \\
\text { aquifer system }\end{array}$ & MSS & nacpmss2 & NACPMSS2-13 & NY & Nassau & $9 / 1,9 / 20 / 2016$ & 253 & NGVD 29 & Public supply & 535 & 470 & 530 \\
\hline $\begin{array}{l}\text { Northern Atlantic } \\
\text { Coastal Plain } \\
\text { aquifer system }\end{array}$ & MSS & nacpmss2 & NACPMSS2-14 & NY & Suffolk & 9/7/2016 & 24 & NAVD 88 & Domestic & 397 & 340 & 392 \\
\hline $\begin{array}{l}\text { Northern Atlantic } \\
\text { Coastal Plain } \\
\text { aquifer system }\end{array}$ & MSS & nacpmss2 & NACPMSS2-15 & NY & Suffolk & 9/7/2016 & 26 & NGVD 29 & Observation & 721 & 668 & 718 \\
\hline $\begin{array}{l}\text { Northern Atlantic } \\
\text { Coastal Plain } \\
\text { aquifer system }\end{array}$ & MSS & nacpmss2 & NACPMSS2-16 & NY & Suffolk & $9 / 8 / 2016$ & 12 & NAVD 88 & Domestic & 250 & 190 & 250 \\
\hline $\begin{array}{l}\text { Northern Atlantic } \\
\text { Coastal Plain } \\
\text { aquifer system }\end{array}$ & MSS & nacpmss2 & NACPMSS2-17 & NY & Suffolk & $9 / 12 / 2016$ & 79 & NGVD 29 & Public supply & 200 & 170 & 200 \\
\hline $\begin{array}{l}\text { Northern Atlantic } \\
\text { Coastal Plain } \\
\text { aquifer system }\end{array}$ & MSS & nacpmss2 & NACPMSS2-18 & NY & Suffolk & $9 / 13 / 2016$ & 26 & NGVD 29 & Public supply & 714 & 650 & 710 \\
\hline $\begin{array}{l}\text { Northern Atlantic } \\
\text { Coastal Plain } \\
\text { aquifer system }\end{array}$ & MSS & nacpmss2 & NACPMSS2-19 & NY & Suffolk & $9 / 14 / 2016$ & 21 & NAVD 88 & Public supply & 708 & 645 & 705 \\
\hline $\begin{array}{l}\text { Northern Atlantic } \\
\text { Coastal Plain } \\
\text { aquifer system }\end{array}$ & MSS & nacpmss2 & NACPMSS2-20 & NY & Suffolk & $9 / 15 / 2016$ & 105 & NGVD 29 & Public supply & 269 & 214 & 265 \\
\hline
\end{tabular}


Table 1. Information about wells that have environmental data included in this report.-Continued

[NAWQA, National Water-Quality Assessment; ID, identification; no., number; LS, land surface; ft bls, foot below land surface; lat., latitude, in degrees and minutes; long., longitude in degrees and minutes; PAS, principal aquifer study; FL, Florida; na, not available; NGVD 29, National Geodetic Vertical Datum of 1929; NAVD 88, North American Vertical Datum of 1988; ETN, enhanced trends network; TX, Texas; NM, New Mexico; LUS, land-use study; SC, South Carolina; FPS, flow-path study; MS, Mississippi; VFPS; vertical flow-path study; TN, Tennessee; OK, Oklahoma; AR, Arkansas; CA, California; MO, Missouri; KS, Kansas; MD, Maryland; MSS, modeling support study; DE, Delaware; DC, District of Columbia; MAS, major aquifer study; NV, Nevada; NY, New York; OH, Ohio; MI, Michigan; ID, Idaho; NH, New Hampshire; WI, Wisconsin; OR, Oregon; MN, Minnesota; WA, Washington]

\begin{tabular}{|c|c|c|c|c|c|c|c|c|c|c|c|c|}
\hline \multirow{2}{*}{$\begin{array}{l}\text { Principal and } \\
\text { regional and (or) } \\
\text { other aquifer } \\
\text { information }\end{array}$} & \multirow{2}{*}{$\begin{array}{l}\text { Network } \\
\text { type }\end{array}$} & \multirow{2}{*}{$\begin{array}{l}\text { Network } \\
\text { name }\end{array}$} & \multirow{2}{*}{$\begin{array}{l}\text { NAWQA well } \\
\text { ID no. }\end{array}$} & \multirow{2}{*}{ State } & \multirow{2}{*}{ County } & \multirow{2}{*}{ Sample date } & \multirow{2}{*}{$\begin{array}{l}\text { Altitude } \\
\text { LS }\end{array}$} & \multirow{2}{*}{$\begin{array}{l}\text { Altitude } \\
\text { datum }\end{array}$} & \multirow{2}{*}{ Water use } & \multirow{2}{*}{$\begin{array}{c}\text { Well } \\
\text { depth } \\
\text { (ft bls) }\end{array}$} & \multicolumn{2}{|c|}{$\begin{array}{l}\text { Depth to perforation } \\
\text { (ft bls) }\end{array}$} \\
\hline & & & & & & & & & & & Top & Bottom \\
\hline $\begin{array}{l}\text { Northern Atlantic } \\
\text { Coastal Plain } \\
\text { aquifer system }\end{array}$ & MSS & nacpmss 2 & NACPMSS2-21 & NY & Suffolk & $9 / 21 / 2016$ & 61 & NAVD 88 & Domestic & 293 & 235 & 290 \\
\hline $\begin{array}{l}\text { Northern Atlantic } \\
\text { Coastal Plain } \\
\text { aquifer system }\end{array}$ & MSS & nacpmss2 & NACPMSS2-22 & NY & Nassau & $9 / 28 / 2016$ & 98 & NGVD 29 & Public supply & 340 & 275 & 335 \\
\hline $\begin{array}{l}\text { Northern Atlantic } \\
\text { Coastal Plain } \\
\text { aquifer system }\end{array}$ & MSS & nacpmss2 & NACPMSS2-23 & NY & Nassau & $10 / 5 / 2016$ & 235 & NAVD 88 & Public supply & 701 & 636 & 696 \\
\hline $\begin{array}{l}\text { Northern Atlantic } \\
\text { Coastal Plain } \\
\text { aquifer system }\end{array}$ & MSS & nacpmss2 & NACPMSS2-24 & NY & Suffolk & $10 / 11 / 2016$ & 113 & NAVD 88 & Public supply & 466 & na & $466^{a}$ \\
\hline $\begin{array}{l}\text { Northern Atlantic } \\
\text { Coastal Plain } \\
\text { aquifer system }\end{array}$ & MSS & nacpmss2 & NACPMSS2-25 & NY & Suffolk & $10 / 18 / 2016$ & 54 & NAVD 88 & Public supply & 303 & 270 & 300 \\
\hline $\begin{array}{l}\text { Ozark Plateaus } \\
\text { aquifer system }\end{array}$ & PAS & ozrkpas1 & OZRKPAS1-01 & OK & Cherokee & $5 / 23 / 2016$ & 965 & NAVD 88 & Public supply & 420 & na & 420 \\
\hline $\begin{array}{l}\text { Ozark Plateaus } \\
\text { aquifer system }\end{array}$ & PAS & ozrkpas1 & OZRKPAS1-02 & OK & Delaware & $5 / 24 / 2016$ & 891 & NAVD 88 & Public supply & 370 & na & $370^{a}$ \\
\hline $\begin{array}{l}\text { Ozark Plateaus } \\
\text { aquifer system }\end{array}$ & PAS & ozrkpas1 & OZRKPAS1-03 & OK & Cherokee & $6 / 13 / 2016$ & 707 & NAVD 88 & Public supply & na & na & na \\
\hline $\begin{array}{l}\text { Ozark Plateaus } \\
\text { aquifer system }\end{array}$ & PAS & ozrkpas1 & OZRKPAS1-04 & OK & Ottawa & $6 / 14 / 2016$ & 810 & NGVD 29 & Public supply & 1,150 & 850 & 1,150 \\
\hline $\begin{array}{l}\text { Ozark Plateaus } \\
\text { aquifer system }\end{array}$ & PAS & ozrkpas 1 & OZRKPAS1-05 & MO & Franklin & $6 / 2 / 2016$ & 905 & NGVD 29 & Public supply & 775 & 314 & 775 \\
\hline $\begin{array}{l}\text { Ozark Plateaus } \\
\text { aquifer system }\end{array}$ & PAS & ozrkpas1 & OZRKPAS1-06 & MO & Stoddard & $4 / 27 / 2016$ & 379 & NAVD 88 & Public supply & 508 & 54 & 497 \\
\hline $\begin{array}{l}\text { Ozark Plateaus } \\
\text { aquifer system }\end{array}$ & PAS & ozrkpas1 & OZRKPAS1-07 & MO & Reynolds & $4 / 28 / 2016$ & 859 & NAVD 88 & Public supply & 670 & 530 & 670 \\
\hline $\begin{array}{l}\text { Ozark Plateaus } \\
\text { aquifer system }\end{array}$ & PAS & ozrkpas1 & OZRKPAS1-08 & MO & Carter & $5 / 3 / 2016$ & 720 & NGVD 29 & Public supply & 1,000 & 128 & 136 \\
\hline
\end{tabular}
aquifer system 
Table 1. Information about wells that have environmental data included in this report.-Continued

[NAWQA, National Water-Quality Assessment; ID, identification; no., number; LS, land surface; $\mathrm{ft}$ bls, foot below land surface; lat., latitude, in degrees and minutes; long., longitude in degrees and minutes; PAS, principal aquifer study; FL, Florida; na, not available; NGVD 29, National Geodetic Vertical Datum of 1929; NAVD 88, North American Vertical Datum of 1988; ETN, enhanced trends network; TX,

Texas; NM, New Mexico; LUS, land-use study; SC, South Carolina; FPS, flow-path study; MS, Mississippi; VFPS; vertical flow-path study; TN, Tennessee; OK, Oklahoma; AR, Arkansas; CA, California; MO, Missouri; KS, Kansas; MD, Maryland; MSS, modeling support study; DE, Delaware; DC, District of Columbia; MAS, major aquifer study; NV, Nevada; NY, New York; OH, Ohio; MI, Michigan; ID, Idaho;

NH, New Hampshire; WI, Wisconsin; OR, Oregon; MN, Minnesota; WA, Washington]

\begin{tabular}{|c|c|c|c|c|c|c|c|c|c|c|c|c|}
\hline \multirow{2}{*}{$\begin{array}{l}\text { Principal and } \\
\text { regional and (or) } \\
\text { other aquifer } \\
\text { information }\end{array}$} & \multirow{2}{*}{$\begin{array}{l}\text { Network } \\
\text { type }\end{array}$} & \multirow{2}{*}{$\begin{array}{l}\text { Network } \\
\text { name }\end{array}$} & \multirow{2}{*}{$\begin{array}{l}\text { NAWQA well } \\
\text { ID no. }\end{array}$} & \multirow{2}{*}{ State } & \multirow{2}{*}{ County } & \multirow{2}{*}{ Sample date } & \multirow{2}{*}{$\begin{array}{l}\text { Altitude } \\
\text { LS }\end{array}$} & \multirow{2}{*}{$\begin{array}{l}\text { Altitude } \\
\text { datum }\end{array}$} & \multirow{2}{*}{ Water use } & \multirow{2}{*}{$\begin{array}{l}\text { Well } \\
\text { depth } \\
\text { (ft bls) }\end{array}$} & \multicolumn{2}{|c|}{$\begin{array}{c}\text { Depth to perforation } \\
\text { (ft bls) }\end{array}$} \\
\hline & & & & & & & & & & & Top & Bottom \\
\hline $\begin{array}{l}\text { Ozark Plateaus } \\
\text { aquifer system }\end{array}$ & PAS & ozrkpas1 & OZRKPAS1-09 & MO & Shannon & $5 / 4 / 2016$ & 986 & NGVD 29 & Public supply & 1,110 & 452 & 1,110 \\
\hline $\begin{array}{l}\text { Ozark Plateaus } \\
\text { aquifer system }\end{array}$ & PAS & ozrkpas1 & OZRKPAS1-10 & MO & Howell & $5 / 17 / 2016$ & 1,055 & NGVD 29 & Public supply & 1,535 & 950 & 1,535 \\
\hline $\begin{array}{l}\text { Ozark Plateaus } \\
\text { aquifer system }\end{array}$ & PAS & ozrkpas 1 & OZRKPAS1-11 & MO & Oregon & $5 / 18 / 2016$ & 755 & NAVD 88 & Public supply & 1,200 & 525 & 1,315 \\
\hline $\begin{array}{l}\text { Ozark Plateaus } \\
\text { aquifer system }\end{array}$ & PAS & ozrkpas1 & OZRKPAS1-12 & MO & Howell & $5 / 19 / 2016$ & 1,414 & NAVD 88 & Public supply & 1,495 & 505 & 1,495 \\
\hline $\begin{array}{l}\text { Ozark Plateaus } \\
\text { aquifer system }\end{array}$ & PAS & ozrkpas1 & OZRKPAS1-13 & MO & Taney & $5 / 23 / 2016$ & 1,060 & NGVD 29 & Public supply & 1,570 & 450 & 1,475 \\
\hline $\begin{array}{l}\text { Ozark Plateaus } \\
\text { aquifer system }\end{array}$ & PAS & ozrkpas1 & OZRKPAS1-14 & MO & Taney & $5 / 24 / 2016$ & 1,010 & NGVD 29 & Public supply & 1,000 & 400 & 1,000 \\
\hline $\begin{array}{l}\text { Ozark Plateaus } \\
\text { aquifer system }\end{array}$ & PAS & ozrkpas1 & OZRKPAS1-15 & MO & Wright & $5 / 25 / 2016$ & 15,30 & NGVD 29 & Public supply & 1,475 & 600 & 1,475 \\
\hline $\begin{array}{l}\text { Ozark Plateaus } \\
\text { aquifer system }\end{array}$ & PAS & ozrkpas1 & OZRKPAS1-16 & MO & Howell & $5 / 26 / 2016$ & 1,165 & NGVD 29 & Public supply & 1,368 & 414 & 1,368 \\
\hline $\begin{array}{l}\text { Ozark Plateaus } \\
\text { aquifer system }\end{array}$ & PAS & ozrkpas1 & OZRKPAS1-17 & MO & Phelps & $5 / 31 / 2016$ & 1,192 & NAVD 88 & Public supply & 1,050 & 425 & 1,050 \\
\hline $\begin{array}{l}\text { Ozark Plateaus } \\
\text { aquifer system }\end{array}$ & PAS & ozrkpas1 & OZRKPAS1-18 & MO & Crawford & $6 / 1 / 2016$ & 1,027 & NAVD 88 & Public supply & 1,050 & 506 & 1,050 \\
\hline $\begin{array}{l}\text { Ozark Plateaus } \\
\text { aquifer system }\end{array}$ & PAS & ozrkpas1 & OZRKPAS1-19 & MO & Texas & $6 / 6 / 2016$ & 1,472 & NGVD 29 & Public supply & 1,100 & 500 & 1,100 \\
\hline $\begin{array}{l}\text { Ozark Plateaus } \\
\text { aquifer system }\end{array}$ & PAS & ozrkpas1 & OZRKPAS1-20 & MO & Gasconade & $6 / 7 / 2016$ & 987 & NGVD 29 & Public supply & 875 & 404 & 875 \\
\hline $\begin{array}{l}\text { Ozark Plateaus } \\
\text { aquifer system }\end{array}$ & PAS & ozrkpas1 & OZRKPAS1-21 & MO & Taney & $6 / 7 / 2016$ & 981 & NAVD 88 & Public supply & 1,025 & 484 & 1,025 \\
\hline $\begin{array}{l}\text { Ozark Plateaus } \\
\text { aquifer system }\end{array}$ & PAS & ozrkpas1 & OZRKPAS1-22 & MO & Franklin & $6 / 8 / 2016$ & 598 & NGVD 29 & Public supply & 900 & 525 & 900 \\
\hline $\begin{array}{l}\text { Ozark Plateaus } \\
\text { aquifer system }\end{array}$ & PAS & ozrkpas1 & OZRKPAS1-23 & MO & Barry & $6 / 8 / 2016$ & 1,497 & NGVD 29 & Public supply & 1,675 & 652 & 1,675 \\
\hline
\end{tabular}


Table 1. Information about wells that have environmental data included in this report.-Continued

[NAWQA, National Water-Quality Assessment; ID, identification; no., number; LS, land surface; ft bls, foot below land surface; lat., latitude, in degrees and minutes; long., longitude in degrees and minutes; PAS, principal aquifer study; FL, Florida; na, not available; NGVD 29, National Geodetic Vertical Datum of 1929; NAVD 88, North American Vertical Datum of 1988; ETN, enhanced trends network; TX, Texas; NM, New Mexico; LUS, land-use study; SC, South Carolina; FPS, flow-path study; MS, Mississippi; VFPS; vertical flow-path study; TN, Tennessee; OK, Oklahoma; AR, Arkansas; CA, California; MO, Missouri; KS, Kansas; MD, Maryland; MSS, modeling support study; DE, Delaware; DC, District of Columbia; MAS, major aquifer study; NV, Nevada; NY, New York; OH, Ohio; MI, Michigan; ID, Idaho; NH, New Hampshire; WI, Wisconsin; OR, Oregon; MN, Minnesota; WA, Washington]

\begin{tabular}{|c|c|c|c|c|c|c|c|c|c|c|c|c|}
\hline \multirow{2}{*}{$\begin{array}{l}\text { Principal and } \\
\text { regional and (or) } \\
\text { other aquifer } \\
\text { information }\end{array}$} & \multirow{2}{*}{$\begin{array}{l}\text { Network } \\
\text { type }\end{array}$} & \multirow{2}{*}{$\begin{array}{l}\text { Network } \\
\text { name }\end{array}$} & \multirow{2}{*}{$\begin{array}{l}\text { NAWQA well } \\
\text { ID no. }\end{array}$} & \multirow{2}{*}{ State } & \multirow{2}{*}{ County } & \multirow{2}{*}{ Sample date } & \multirow{2}{*}{$\begin{array}{c}\text { Altitude } \\
\text { LS }\end{array}$} & \multirow{2}{*}{$\begin{array}{l}\text { Altitude } \\
\text { datum }\end{array}$} & \multirow{2}{*}{ Water use } & \multirow{2}{*}{$\begin{array}{c}\text { Well } \\
\text { depth } \\
\text { (ft bls) }\end{array}$} & \multicolumn{2}{|c|}{$\begin{array}{c}\text { Depth to perforation } \\
\text { (ft bls) }\end{array}$} \\
\hline & & & & & & & & & & & Top & Bottom \\
\hline $\begin{array}{l}\text { Ozark Plateaus } \\
\text { aquifer system }\end{array}$ & PAS & ozrkpas 1 & OZRKPAS1-24 & $\mathrm{MO}$ & Jasper & $6 / 9 / 2016$ & 1,054 & NAVD 88 & Public supply & 1,875 & na & $1,875^{\mathrm{a}}$ \\
\hline $\begin{array}{l}\text { Ozark Plateaus } \\
\text { aquifer system }\end{array}$ & PAS & ozrkpas1 & OZRKPAS1-25 & $\mathrm{MO}$ & Pulaski & $6 / 9 / 2016$ & 1,110 & NGVD 29 & Public supply & 1,202 & 335 & 1,202 \\
\hline $\begin{array}{l}\text { Ozark Plateaus } \\
\text { aquifer system }\end{array}$ & PAS & ozrkpas1 & OZRKPAS1-26 & $\mathrm{MO}$ & Jasper & $6 / 14 / 2016$ & 1,149 & NAVD 88 & Public supply & 1,500 & 453 & 1,500 \\
\hline $\begin{array}{l}\text { Ozark Plateaus } \\
\text { aquifer system }\end{array}$ & PAS & ozrkpas1 & OZRKPAS1-27 & $\mathrm{MO}$ & Texas & $6 / 14 / 2016$ & 1,267 & NGVD 29 & Public supply & 931 & 310 & 931 \\
\hline $\begin{array}{l}\text { Ozark Plateaus } \\
\text { aquifer system }\end{array}$ & PAS & ozrkpas 1 & OZRKPAS1-28 & $\mathrm{MO}$ & Barry & $6 / 15 / 2016$ & 1,480 & NGVD 29 & Public supply & 1,425 & 485 & 1,425 \\
\hline $\begin{array}{l}\text { Ozark Plateaus } \\
\text { aquifer system }\end{array}$ & PAS & ozrkpas 1 & OZRKPAS1-29 & $\mathrm{MO}$ & Dent & $6 / 15,7 / 7 / 2016$ & 1,380 & NGVD 29 & Public supply & 850 & 310 & 850 \\
\hline $\begin{array}{l}\text { Ozark Plateaus } \\
\text { aquifer system }\end{array}$ & PAS & ozrkpas1 & OZRKPAS1-30 & $\mathrm{MO}$ & McDonald & $6 / 16 / 2016$ & 1,040 & NGVD 29 & Public supply & 1,225 & 225 & 1,300 \\
\hline $\begin{array}{l}\text { Ozark Plateaus } \\
\text { aquifer system }\end{array}$ & PAS & ozrkpas1 & OZRKPAS1-31 & $\mathrm{MO}$ & Webster & $6 / 27 / 2016$ & 1,490 & NGVD 29 & Public supply & 1,420 & 425 & 1,420 \\
\hline $\begin{array}{l}\text { Ozark Plateaus } \\
\text { aquifer system }\end{array}$ & PAS & ozrkpas 1 & OZRKPAS1-32 & MO & Cole & $6 / 29 / 2016$ & 815 & NAVD 88 & Public supply & 1,100 & 400 & 1,100 \\
\hline $\begin{array}{l}\text { Ozark Plateaus } \\
\text { aquifer system }\end{array}$ & PAS & ozrkpas 1 & OZRKPAS1-33 & MO & Cole & $6 / 30 / 2016$ & 795 & NGVD 29 & Public supply & 730 & 400 & 730 \\
\hline $\begin{array}{l}\text { Ozark Plateaus } \\
\text { aquifer system }\end{array}$ & PAS & ozrkpas1 & OZRKPAS1-34 & $\mathrm{MO}$ & Jefferson & $7 / 6,8 / 17 / 2016$ & 800 & NGVD 29 & Public supply & 1,200 & 480 & 1,200 \\
\hline $\begin{array}{l}\text { Ozark Plateaus } \\
\text { aquifer system }\end{array}$ & PAS & ozrkpas1 & OZRKPAS1-35 & MO & Barton & $7 / 11 / 2016$ & 995 & NAVD 88 & Public supply & 1,200 & 603 & 1,200 \\
\hline $\begin{array}{l}\text { Ozark Plateaus } \\
\text { aquifer system }\end{array}$ & PAS & ozrkpas 1 & OZRKPAS1-36 & $\mathrm{MO}$ & Barton & $7 / 12 / 2016$ & 1,020 & NGVD 29 & Public supply & 940 & 550 & 940 \\
\hline $\begin{array}{l}\text { Ozark Plateaus } \\
\text { aquifer system }\end{array}$ & PAS & ozrkpas1 & OZRKPAS1-37 & $\mathrm{MO}$ & Vernon & $7 / 13 / 2016$ & 985 & NAVD 88 & Public supply & 1,115 & 560 & 1,115 \\
\hline $\begin{array}{l}\text { Ozark Plateaus } \\
\text { aquifer system }\end{array}$ & PAS & ozrkpas1 & OZRKPAS1-38 & MO & St. Clair & $7 / 14 / 2016$ & 822 & NGVD 29 & Public supply & 852 & 252 & 852 \\
\hline
\end{tabular}


Table 1. Information about wells that have environmental data included in this report.-Continued

[NAWQA, National Water-Quality Assessment; ID, identification; no., number; LS, land surface; ft bls, foot below land surface; lat., latitude, in degrees and minutes; long., longitude in degrees and minutes; PAS, principal aquifer study; FL, Florida; na, not available; NGVD 29, National Geodetic Vertical Datum of 1929; NAVD 88, North American Vertical Datum of 1988; ETN, enhanced trends network; TX,

Texas; NM, New Mexico; LUS, land-use study; SC, South Carolina; FPS, flow-path study; MS, Mississippi; VFPS; vertical flow-path study; TN, Tennessee; OK, Oklahoma; AR, Arkansas; CA, California; MO, Missouri; KS, Kansas; MD, Maryland; MSS, modeling support study; DE, Delaware; DC, District of Columbia; MAS, major aquifer study; NV, Nevada; NY, New York; OH, Ohio; MI, Michigan; ID, Idaho;

NH, New Hampshire; WI, Wisconsin; OR, Oregon; MN, Minnesota; WA, Washington]

\begin{tabular}{|c|c|c|c|c|c|c|c|c|c|c|c|c|}
\hline \multirow{2}{*}{$\begin{array}{l}\text { Principal and } \\
\text { regional and (or) } \\
\text { other aquifer } \\
\text { information }\end{array}$} & \multirow{2}{*}{$\begin{array}{l}\text { Network } \\
\text { type }\end{array}$} & \multirow{2}{*}{$\begin{array}{l}\text { Network } \\
\text { name }\end{array}$} & \multirow{2}{*}{$\begin{array}{l}\text { NAWQA well } \\
\text { ID no. }\end{array}$} & \multirow{2}{*}{ State } & \multirow{2}{*}{ County } & \multirow{2}{*}{ Sample date } & \multirow{2}{*}{$\begin{array}{c}\text { Altitude } \\
\text { LS }\end{array}$} & \multirow{2}{*}{$\begin{array}{l}\text { Altitude } \\
\text { datum }\end{array}$} & \multirow{2}{*}{ Water use } & \multirow{2}{*}{$\begin{array}{l}\text { Well } \\
\text { depth } \\
\text { (ft bls) }\end{array}$} & \multicolumn{2}{|c|}{$\begin{array}{c}\text { Depth to perforation } \\
\text { (ft bls) }\end{array}$} \\
\hline & & & & & & & & & & & Top & Bottom \\
\hline $\begin{array}{l}\text { Ozark Plateaus } \\
\text { aquifer system }\end{array}$ & PAS & ozrkpas1 & OZRKPAS1-39 & $\mathrm{MO}$ & Hickory & $7 / 18 / 2016$ & 277 & NGVD 29 & Public supply & 1,002 & 455 & 1,002 \\
\hline $\begin{array}{l}\text { Ozark Plateaus } \\
\text { aquifer system }\end{array}$ & PAS & ozrkpas1 & OZRKPAS1-40 & $\mathrm{MO}$ & Dallas & $7 / 19 / 2016$ & 1,195 & NAVD 88 & Public supply & 1,225 & 477 & 1,225 \\
\hline $\begin{array}{l}\text { Ozark Plateaus } \\
\text { aquifer system }\end{array}$ & PAS & ozrkpas 1 & OZRKPAS1-41 & $\mathrm{MO}$ & Christian & $7 / 20 / 2016$ & 1,340 & NGVD 29 & Public supply & 1,375 & 450 & 1,375 \\
\hline $\begin{array}{l}\text { Ozark Plateaus } \\
\text { aquifer system }\end{array}$ & PAS & ozrkpas1 & OZRKPAS1-42 & $\mathrm{MO}$ & Camden & $7 / 21 / 2016$ & 1,040 & NGVD 29 & Public supply & 597 & 425 & 597 \\
\hline $\begin{array}{l}\text { Ozark Plateaus } \\
\text { aquifer system }\end{array}$ & PAS & ozrkpas 1 & OZRKPAS1-43 & $\mathrm{MO}$ & Morgan & $7 / 25 / 2016$ & 1,070 & NGVD 29 & Public supply & 525 & 161 & 525 \\
\hline $\begin{array}{l}\text { Ozark Plateaus } \\
\text { aquifer system }\end{array}$ & PAS & ozrkpas 1 & OZRKPAS1-44 & $\mathrm{MO}$ & Benton & $7 / 26 / 2016$ & 952 & NAVD 88 & Public supply & 1,155 & 450 & 1,155 \\
\hline $\begin{array}{l}\text { Ozark Plateaus } \\
\text { aquifer system }\end{array}$ & PAS & ozrkpas1 & OZRKPAS1-45 & $\mathrm{MO}$ & Pettis & $7 / 27 / 2016$ & 865 & NGVD 29 & Public supply & 1,105 & 350 & 1,105 \\
\hline $\begin{array}{l}\text { Ozark Plateaus } \\
\text { aquifer system }\end{array}$ & PAS & ozrkpas1 & OZRKPAS1-46 & $\mathrm{MO}$ & Cooper & $7 / 28 / 2016$ & 855 & NGVD 29 & Public supply & 630 & 300 & 630 \\
\hline $\begin{array}{l}\text { Ozark Plateaus } \\
\text { aquifer system }\end{array}$ & PAS & ozrkpas 1 & OZRKPAS1-47 & MO & Laclede & $8 / 16 / 2016$ & 1,357 & NAVD 88 & Public supply & 1,250 & 630 & 1,250 \\
\hline $\begin{array}{l}\text { Ozark Plateaus } \\
\text { aquifer system }\end{array}$ & PAS & ozrkpas 1 & OZRKPAS1-48 & $\mathrm{MO}$ & Perry & $8 / 22,8 / 24 / 2016$ & 652 & NAVD 88 & Public supply & 500 & 350 & 500 \\
\hline $\begin{array}{l}\text { Ozark Plateaus } \\
\text { aquifer system }\end{array}$ & PAS & ozrkpas1 & OZRKPAS1-49 & MO & $\begin{array}{l}\text { Cape } \\
\text { Girardeau }\end{array}$ & $8 / 23 / 2016$ & 450 & NGVD 29 & Public supply & 2,217 & 356 & 2,217 \\
\hline $\begin{array}{l}\text { Ozark Plateaus } \\
\text { aquifer system }\end{array}$ & PAS & ozrkpas1 & OZRKPAS1-50 & $\mathrm{MO}$ & $\begin{array}{l}\text { Cape } \\
\text { Girardeau }\end{array}$ & $8 / 24 / 2016$ & na & na & Public supply & 1,318 & 386 & 1,318 \\
\hline $\begin{array}{l}\text { Ozark Plateaus } \\
\text { aquifer system }\end{array}$ & PAS & ozrkpas 1 & OZRKPAS1-51 & MO & $\begin{array}{l}\text { Ste. } \\
\text { Genevieve }\end{array}$ & $8 / 25 / 2016$ & 770 & NGVD 29 & Public supply & 1,700 & 500 & 1,500 \\
\hline $\begin{array}{l}\text { Ozark Plateaus } \\
\text { aquifer system }\end{array}$ & PAS & ozrkpas1 & OZRKPAS1-52 & $\mathrm{AR}$ & Lawrence & $4 / 25 / 2016$ & 318 & NGVD 29 & Public supply & 526 & 40 & 527 \\
\hline $\begin{array}{l}\text { Ozark Plateaus } \\
\text { aquifer system }\end{array}$ & PAS & ozrkpas1 & OZRKPAS1-53 & $\mathrm{AR}$ & Sharp & $4 / 26 / 2016$ & 653 & NAVD 88 & Public supply & 1,525 & 500 & 1,525 \\
\hline
\end{tabular}


Table 1. Information about wells that have environmental data included in this report.-Continued

[NAWQA, National Water-Quality Assessment; ID, identification; no., number; LS, land surface; ft bls, foot below land surface; lat., latitude, in degrees and minutes; long., longitude in degrees and minutes; PAS, principal aquifer study; FL, Florida; na, not available; NGVD 29, National Geodetic Vertical Datum of 1929; NAVD 88, North American Vertical Datum of 1988; ETN, enhanced trends network; TX, Texas; NM, New Mexico; LUS, land-use study; SC, South Carolina; FPS, flow-path study; MS, Mississippi; VFPS; vertical flow-path study; TN, Tennessee; OK, Oklahoma; AR, Arkansas; CA, California; MO, Missouri; KS, Kansas; MD, Maryland; MSS, modeling support study; DE, Delaware; DC, District of Columbia; MAS, major aquifer study; NV, Nevada; NY, New York; OH, Ohio; MI, Michigan; ID, Idaho;

NH, New Hampshire; WI, Wisconsin; OR, Oregon; MN, Minnesota; WA, Washington]

\begin{tabular}{|c|c|c|c|c|c|c|c|c|c|c|c|c|}
\hline \multirow{2}{*}{$\begin{array}{l}\text { Principal and } \\
\text { regional and (or) } \\
\text { other aquifer } \\
\text { information }\end{array}$} & \multirow{2}{*}{$\begin{array}{l}\text { Network } \\
\text { type }\end{array}$} & \multirow{2}{*}{$\begin{array}{l}\text { Network } \\
\text { name }\end{array}$} & \multirow{2}{*}{$\begin{array}{l}\text { NAWQA well } \\
\text { ID no. }\end{array}$} & \multirow{2}{*}{ State } & \multirow{2}{*}{ County } & \multirow{2}{*}{ Sample date } & \multirow{2}{*}{$\begin{array}{l}\text { Altitude } \\
\text { LS }\end{array}$} & \multirow{2}{*}{$\begin{array}{c}\text { Altitude } \\
\text { datum }\end{array}$} & \multirow{2}{*}{ Water use } & \multirow{2}{*}{$\begin{array}{r}\text { Well } \\
\text { depth } \\
\text { (ft bls) }\end{array}$} & \multicolumn{2}{|c|}{$\begin{array}{c}\text { Depth to perforation } \\
\text { (ft bls) }\end{array}$} \\
\hline & & & & & & & & & & & Top & Bottom \\
\hline $\begin{array}{l}\text { Ozark Plateaus } \\
\text { aquifer system }\end{array}$ & PAS & ozrkpas 1 & OZRKPAS1-54 & $\mathrm{AR}$ & Sharp & $5 / 2 / 2016$ & 1,667 & NGVD 29 & Public supply & na & na & na \\
\hline $\begin{array}{l}\text { Ozark Plateaus } \\
\text { aquifer system }\end{array}$ & PAS & ozrkpas 1 & OZRKPAS1-55 & AR & Stone & $5 / 5 / 2016$ & 980 & NGVD 29 & Public supply & 3,420 & na & $3,420^{a}$ \\
\hline $\begin{array}{l}\text { Ozark Plateaus } \\
\text { aquifer system }\end{array}$ & PAS & ozrkpas1 & OZRKPAS1-56 & $\mathrm{AR}$ & Baxter & $5 / 31 / 2016$ & 720 & NAVD 88 & Public supply & 1,625 & 300 & 1,625 \\
\hline $\begin{array}{l}\text { Ozark Plateaus } \\
\text { aquifer system }\end{array}$ & PAS & ozrkpas 1 & OZRKPAS1-57 & $\mathrm{AR}$ & Boone & $6 / 1 / 2016$ & 1,146 & NAVD 88 & Public supply & 1,649 & na & $1,649 \mathrm{a}$ \\
\hline $\begin{array}{l}\text { Ozark Plateaus } \\
\text { aquifer system }\end{array}$ & PAS & ozrkpas 1 & OZRKPAS1-58 & $\mathrm{AR}$ & Newton & $6 / 1 / 2016$ & $1,344.50$ & NGVD 29 & Public supply & 2,576 & 607.5 & 2,576 \\
\hline $\begin{array}{l}\text { Ozark Plateaus } \\
\text { aquifer system }\end{array}$ & PAS & ozrkpas 1 & OZRKPAS1-59 & $\mathrm{AR}$ & Madison & $6 / 6 / 2016$ & 1,446 & NGVD 29 & Public supply & na & na & na \\
\hline $\begin{array}{l}\text { Ozark Plateaus } \\
\text { aquifer system }\end{array}$ & PAS & ozrkpas 1 & OZRKPAS1-60 & KS & Crawford & $6 / 13 / 2016$ & 887 & NGVD 29 & Public supply & 1,052 & na & $1,052^{\mathrm{a}}$ \\
\hline $\begin{array}{l}\text { Rio Grande aqui- } \\
\text { fer system }\end{array}$ & ETN & rgaqetn1 & RGAQETN1-02 & NM & Dona Ana & $5 / 10 / 2016$ & 4,058 & NAVD 88 & Other & 60 & 40 & 60 \\
\hline $\begin{array}{l}\text { Rio Grande aqui- } \\
\text { fer system }\end{array}$ & ETN & rgaqetn1 & RGAQETN1-03 & NM & Sierra & $5 / 9 / 2016$ & 4,134 & NAVD 88 & Observation & 22 & 11.5 & 21.5 \\
\hline $\begin{array}{l}\text { Rio Grande aqui- } \\
\text { fer system }\end{array}$ & LUS & rioglusag1 & RIOGLUSAG1-01 & NM & Dona Ana & $4 / 4 / 2016$ & $4,000.70$ & NAVD 88 & Observation & 15.1 & 5.1 & 14.4 \\
\hline $\begin{array}{l}\text { Rio Grande aqui- } \\
\text { fer system }\end{array}$ & LUS & rioglusag1 & RIOGLUSAG1-02 & NM & Dona Ana & $4 / 4 / 2016$ & $4,005.10$ & NAVD 88 & Observation & 14.9 & 4.9 & 14.3 \\
\hline $\begin{array}{l}\text { Rio Grande aqui- } \\
\text { fer system }\end{array}$ & LUS & rioglusag1 & RIOGLUSAG1-03 & NM & Dona Ana & $4 / 5 / 2016$ & $4,015.40$ & NAVD 88 & Observation & 23 & 13.1 & 22.4 \\
\hline $\begin{array}{l}\text { Rio Grande aqui- } \\
\text { fer system }\end{array}$ & LUS & rioglusag1 & RIOGLUSAG1-04 & NM & Dona Ana & $4 / 5 / 2016$ & $4,023.40$ & NAVD 88 & Observation & 16.9 & 6.9 & 16.2 \\
\hline $\begin{array}{l}\text { Rio Grande aqui- } \\
\text { fer system }\end{array}$ & LUS & rioglusag1 & RIOGLUSAG1-05 & NM & Dona Ana & $4 / 5 / 2016$ & $4,028.40$ & NAVD 88 & Observation & 18.3 & 8.3 & 17.6 \\
\hline $\begin{array}{l}\text { Rio Grande aqui- } \\
\text { fer system }\end{array}$ & LUS & rioglusag1 & RIOGLUSAG1-06 & NM & Dona Ana & $4 / 5 / 2016$ & $4,028.40$ & NAVD 88 & Observation & 18.2 & 8.2 & 17.5 \\
\hline
\end{tabular}


Table 1. Information about wells that have environmental data included in this report.-Continued

[NAWQA, National Water-Quality Assessment; ID, identification; no., number; LS, land surface; $\mathrm{ft}$ bls, foot below land surface; lat., latitude, in degrees and minutes; long., longitude in degrees and minutes; PAS, principal aquifer study; FL, Florida; na, not available; NGVD 29, National Geodetic Vertical Datum of 1929; NAVD 88, North American Vertical Datum of 1988; ETN, enhanced trends network; TX,

Texas; NM, New Mexico; LUS, land-use study; SC, South Carolina; FPS, flow-path study; MS, Mississippi; VFPS; vertical flow-path study; TN, Tennessee; OK, Oklahoma; AR, Arkansas; CA, California; MO, Missouri; KS, Kansas; MD, Maryland; MSS, modeling support study; DE, Delaware; DC, District of Columbia; MAS, major aquifer study; NV, Nevada; NY, New York; OH, Ohio; MI, Michigan; ID, Idaho;

NH, New Hampshire; WI, Wisconsin; OR, Oregon; MN, Minnesota; WA, Washington]

\begin{tabular}{|c|c|c|c|c|c|c|c|c|c|c|c|c|}
\hline \multirow{2}{*}{$\begin{array}{l}\text { Principal and } \\
\text { regional and (or) } \\
\text { other aquifer } \\
\text { information }\end{array}$} & \multirow{2}{*}{$\begin{array}{l}\text { Network } \\
\text { type }\end{array}$} & \multirow{2}{*}{$\begin{array}{l}\text { Network } \\
\text { name }\end{array}$} & \multirow{2}{*}{$\begin{array}{l}\text { NAWQA well } \\
\text { ID no. }\end{array}$} & \multirow{2}{*}{ State } & \multirow{2}{*}{ County } & \multirow{2}{*}{ Sample date } & \multirow{2}{*}{$\begin{array}{c}\text { Altitude } \\
\text { LS }\end{array}$} & \multirow{2}{*}{$\begin{array}{l}\text { Altitude } \\
\text { datum }\end{array}$} & \multirow{2}{*}{ Water use } & \multirow{2}{*}{$\begin{array}{c}\text { Well } \\
\text { depth } \\
\text { (ft bls) }\end{array}$} & \multicolumn{2}{|c|}{$\begin{array}{c}\text { Depth to perforation } \\
\text { (ft bls) }\end{array}$} \\
\hline & & & & & & & & & & & Top & Bottom \\
\hline $\begin{array}{l}\text { Rio Grande aqui- } \\
\text { fer system }\end{array}$ & LUS & rioglusag1 & RIOGLUSAG1-07 & $\mathrm{NM}$ & Dona Ana & $4 / 6 / 2016$ & 4,071 & NAVD 88 & Observation & 28.1 & 13.5 & 23.5 \\
\hline $\begin{array}{l}\text { Rio Grande aqui- } \\
\text { fer system }\end{array}$ & LUS & rioglusag1 & RIOGLUSAG1-08 & $\mathrm{NM}$ & Dona Ana & $4 / 6 / 2016$ & $4,057.40$ & NAVD 88 & Observation & 16.1 & 6.1 & 15.4 \\
\hline $\begin{array}{l}\text { Rio Grande aqui- } \\
\text { fer system }\end{array}$ & LUS & rioglusag1 & RIOGLUSAG1-09 & NM & Dona Ana & $4 / 6 / 2016$ & $4,081.50$ & NAVD 88 & Observation & 24.2 & 14.2 & 23.6 \\
\hline $\begin{array}{l}\text { Rio Grande aqui- } \\
\text { fer system }\end{array}$ & LUS & rioglusag1 & RIOGLUSAG1-10 & $\mathrm{NM}$ & Dona Ana & $4 / 6 / 2016$ & $4,069.10$ & NAVD 88 & Observation & 19.9 & 9.9 & 19.3 \\
\hline $\begin{array}{l}\text { Rio Grande aqui- } \\
\text { fer system }\end{array}$ & LUS & rioglusag1 & RIOGLUSAG1-11 & NM & Sierra & $4 / 7 / 2016$ & $4,118.60$ & NAVD 88 & Observation & 26.4 & 9 & 19 \\
\hline $\begin{array}{l}\text { Rio Grande aqui- } \\
\text { fer system }\end{array}$ & LUS & rioglusag1 & RIOGLUSAG1-12 & NM & Sierra & $4 / 7 / 2016$ & $4,132.60$ & NAVD 88 & Observation & 17.5 & 7.5 & 16.9 \\
\hline $\begin{array}{l}\text { Rio Grande aqui- } \\
\text { fer system }\end{array}$ & LUS & rioglusag1 & RIOGLUSAG1-13 & NM & Sierra & $4 / 7 / 2016$ & $4,119.90$ & NAVD 88 & Observation & 32.6 & 15 & 25 \\
\hline $\begin{array}{l}\text { Rio Grande aqui- } \\
\text { fer system }\end{array}$ & LUS & rioglusag1 & RIOGLUSAG1-14 & NM & Sierra & $4 / 12 / 2016$ & $4,135.30$ & NAVD 88 & Observation & 20.3 & 10.3 & 19.7 \\
\hline $\begin{array}{l}\text { Rio Grande aqui- } \\
\text { fer system }\end{array}$ & LUS & rioglusag1 & RIOGLUSAG1-15 & NM & Sierra & $4 / 12 / 2016$ & $4,138.70$ & NAVD 88 & Observation & 20.9 & 10.9 & 20.2 \\
\hline $\begin{array}{l}\text { Rio Grande aqui- } \\
\text { fer system }\end{array}$ & LUS & rioglusag1 & RIOGLUSAG1-16 & NM & Sierra & $4 / 13 / 2016$ & $4,138.20$ & NAVD 88 & Observation & 21.8 & 11.9 & 21.2 \\
\hline \multirow[t]{2}{*}{$\begin{array}{l}\text { Rio Grande aqui- } \\
\text { fer system }\end{array}$} & LUS/ & $\begin{array}{l}\text { rioglus- } \\
\text { ag1/ }\end{array}$ & RIOGLUSAG1-17/ & NM & Dona Ana & $5 / 10 / 2016$ & $4,015.30$ & NAVD 88 & Observation & 22.6 & 7.5 & 17.5 \\
\hline & ETN & rgaqetn1 & RGAQETN1-01 & & & & & & & & & \\
\hline $\begin{array}{l}\text { Rio Grande aqui- } \\
\text { fer system }\end{array}$ & LUS & rioglusag1 & RIOGLUSAG1-18 & $\mathrm{NM}$ & Sierra & $5 / 16 / 2016$ & 4,133 & NAVD 88 & Observation & 33.5 & 23.3 & 32.9 \\
\hline $\begin{array}{l}\text { Rio Grande aqui- } \\
\text { fer system }\end{array}$ & LUS & rioglusag1 & RIOGLUSAG1-19 & NM & Dona Ana & $5 / 16 / 2016$ & 4,055 & NAVD 88 & Observation & 32.2 & 22.2 & 31.8 \\
\hline $\begin{array}{l}\text { Rio Grande aqui- } \\
\text { fer system }\end{array}$ & LUS & rioglusag1 & RIOGLUSAG1-20 & NM & Dona Ana & $5 / 17 / 2016$ & 4,100 & NAVD 88 & Observation & 33.7 & 23.7 & 33.3 \\
\hline
\end{tabular}


Table 1. Information about wells that have environmental data included in this report.-Continued

[NAWQA, National Water-Quality Assessment; ID, identification; no., number; LS, land surface; ft bls, foot below land surface; lat., latitude, in degrees and minutes; long., longitude in degrees and minutes; PAS, principal aquifer study; FL, Florida; na, not available; NGVD 29, National Geodetic Vertical Datum of 1929; NAVD 88, North American Vertical Datum of 1988; ETN, enhanced trends network; TX, Texas; NM, New Mexico; LUS, land-use study; SC, South Carolina; FPS, flow-path study; MS, Mississippi; VFPS; vertical flow-path study; TN, Tennessee; OK, Oklahoma; AR, Arkansas; CA, California; MO, Missouri; KS, Kansas; MD, Maryland; MSS, modeling support study; DE, Delaware; DC, District of Columbia; MAS, major aquifer study; NV, Nevada; NY, New York; OH, Ohio; MI, Michigan; ID, Idaho; NH, New Hampshire; WI, Wisconsin; OR, Oregon; MN, Minnesota; WA, Washington]

\begin{tabular}{|c|c|c|c|c|c|c|c|c|c|c|c|c|}
\hline \multirow{2}{*}{$\begin{array}{l}\text { Principal and } \\
\text { regional and (or) } \\
\text { other aquifer } \\
\text { information }\end{array}$} & \multirow{2}{*}{$\begin{array}{l}\text { Network } \\
\text { type }\end{array}$} & \multirow{2}{*}{$\begin{array}{l}\text { Network } \\
\text { name }\end{array}$} & \multirow{2}{*}{$\begin{array}{l}\text { NAWQA well } \\
\text { ID no. }\end{array}$} & \multirow{2}{*}{ State } & \multirow{2}{*}{ County } & \multirow{2}{*}{ Sample date } & \multirow{2}{*}{$\begin{array}{l}\text { Altitude } \\
\text { LS }\end{array}$} & \multirow{2}{*}{$\begin{array}{l}\text { Altitude } \\
\text { datum }\end{array}$} & \multirow{2}{*}{ Water use } & \multirow{2}{*}{$\begin{array}{l}\text { Well } \\
\text { depth } \\
\text { (ft bls) }\end{array}$} & \multicolumn{2}{|c|}{$\begin{array}{c}\text { Depth to perforation } \\
\text { (ft bls) }\end{array}$} \\
\hline & & & & & & & & & & & Top & Bottom \\
\hline $\begin{array}{l}\text { Rio Grande aqui- } \\
\text { fer system }\end{array}$ & LUS & rioglusag1 & RIOGLUSAG1-21 & $\mathrm{NM}$ & Dona Ana & $5 / 17 / 2016$ & 4,104 & NAVD 88 & Observation & 37.2 & 27.2 & 36.8 \\
\hline $\begin{array}{l}\text { Rio Grande aqui- } \\
\text { fer system }\end{array}$ & LUS & rioglusag1 & RIOGLUSAG1-22 & $\mathrm{NM}$ & Dona Ana & $5 / 18 / 2016$ & 4,110 & NAVD 88 & Observation & 34.5 & 24.5 & 34.1 \\
\hline $\begin{array}{l}\text { Rio Grande aqui- } \\
\text { fer system }\end{array}$ & LUS & rioglusag1 & RIOGLUSAG1-23 & NM & Dona Ana & $5 / 18 / 2016$ & 4,078 & NAVD 88 & Observation & 37 & 27 & 36.6 \\
\hline $\begin{array}{l}\text { Rio Grande aqui- } \\
\text { fer system }\end{array}$ & LUS & rioglusag1 & RIOGLUSAG1-24 & $\mathrm{NM}$ & Dona Ana & $4 / 12 / 2016$ & $4,047.40$ & NAVD 88 & Observation & 22.9 & 12.9 & 22.2 \\
\hline $\begin{array}{l}\text { Rio Grande aqui- } \\
\text { fer system }\end{array}$ & $\begin{array}{l}\text { LUS/ } \\
\text { VFPS }\end{array}$ & $\begin{array}{l}\text { rioglusrc1/ } \\
\text { rgaqvfps1 }\end{array}$ & $\begin{array}{l}\text { RIOGLUSRC1-01/ } \\
\text { RGAQVFPS1-04 }\end{array}$ & NM & Bernalillo & $7 / 11 / 2016$ & 4,991 & NGVD 29 & Observation & 44 & 26 & 41 \\
\hline $\begin{array}{l}\text { Rio Grande aqui- } \\
\text { fer system }\end{array}$ & $\begin{array}{l}\text { LUS/ } \\
\text { VFPS }\end{array}$ & $\begin{array}{l}\text { rioglusrc1/ } \\
\text { rgaqvfps1 }\end{array}$ & $\begin{array}{l}\text { RIOGLUSRC1-02/ } \\
\text { RGAQVFPS1-13 }\end{array}$ & $\mathrm{NM}$ & Bernalillo & $7 / 12 / 2016$ & 4,965 & NGVD 29 & Observation & 26.5 & 16 & 26 \\
\hline $\begin{array}{l}\text { Rio Grande aqui- } \\
\text { fer system }\end{array}$ & $\begin{array}{l}\text { LUS/ } \\
\text { VFPS }\end{array}$ & $\begin{array}{l}\text { rioglusrc1/ } \\
\text { rgaqvfps1 }\end{array}$ & $\begin{array}{l}\text { RIOGLUSRC1-03/ } \\
\text { RGAQVFPS1-19 }\end{array}$ & $\mathrm{NM}$ & Bernalillo & $7 / 12 / 2016$ & 4,927 & NGVD 29 & Observation & 20.2 & 7.5 & 17.5 \\
\hline $\begin{array}{l}\text { Rio Grande aqui- } \\
\text { fer system }\end{array}$ & $\begin{array}{l}\text { LUS/ } \\
\text { VFPS }\end{array}$ & $\begin{array}{l}\text { rioglusrc1/ } \\
\text { rgaqvfps1 }\end{array}$ & $\begin{array}{l}\text { RIOGLUSRC1-04/ } \\
\text { RGAQVFPS1-15 }\end{array}$ & $\mathrm{NM}$ & Bernalillo & $7 / 13 / 2016$ & 4,945 & NGVD 29 & Observation & 28 & 18 & 28 \\
\hline $\begin{array}{l}\text { Rio Grande aqui- } \\
\text { fer system }\end{array}$ & $\begin{array}{l}\text { LUS/ } \\
\text { VFPS }\end{array}$ & $\begin{array}{l}\text { rioglusrc1/ } \\
\text { rgaqvfps1 }\end{array}$ & $\begin{array}{l}\text { RIOGLUSRC1-05/ } \\
\text { RGAQVFPS1-18 }\end{array}$ & $\mathrm{NM}$ & Bernalillo & $7 / 13 / 2016$ & 4,935 & NGVD 29 & Observation & 18.3 & 7.8 & 17.8 \\
\hline $\begin{array}{l}\text { Rio Grande aqui- } \\
\text { fer system }\end{array}$ & $\begin{array}{l}\text { LUS/ } \\
\text { VFPS }\end{array}$ & $\begin{array}{l}\text { rioglusrc1/ } \\
\text { rgaqvfps1 }\end{array}$ & $\begin{array}{l}\text { RIOGLUSRC1-06/ } \\
\text { RGAQVFPS1-02 }\end{array}$ & $\mathrm{NM}$ & Bernalillo & $7 / 13 / 2016$ & 4,990 & NGVD 29 & Observation & 23.4 & 13 & 23 \\
\hline $\begin{array}{l}\text { Rio Grande aqui- } \\
\text { fer system }\end{array}$ & $\begin{array}{l}\text { LUS/ } \\
\text { VFPS }\end{array}$ & $\begin{array}{l}\text { rioglusrc1/ } \\
\text { rgaqvfps1 }\end{array}$ & $\begin{array}{l}\text { RIOGLUSRC1-07/ } \\
\text { RGAQVFPS1-16 }\end{array}$ & NM & Bernalillo & $7 / 13 / 2016$ & 4,940 & NGVD 29 & Observation & 19.7 & 9.5 & 19.5 \\
\hline $\begin{array}{l}\text { Rio Grande aqui- } \\
\text { fer system }\end{array}$ & $\begin{array}{l}\text { LUS/ } \\
\text { VFPS }\end{array}$ & $\begin{array}{l}\text { rioglusrc1/ } \\
\text { rgaqvfps1 }\end{array}$ & $\begin{array}{l}\text { RIOGLUSRC1-08/ } \\
\text { RGAQVFPS1-11 }\end{array}$ & $\mathrm{NM}$ & Bernalillo & $7 / 14 / 2016$ & 4,967 & NGVD 29 & Observation & 38.5 & 21 & 36 \\
\hline $\begin{array}{l}\text { Rio Grande aqui- } \\
\text { fer system }\end{array}$ & $\begin{array}{l}\text { LUS/ } \\
\text { VFPS }\end{array}$ & $\begin{array}{l}\text { rioglusrc1/ } \\
\text { rgaqvfps1 }\end{array}$ & $\begin{array}{l}\text { RIOGLUSRC1-09/ } \\
\text { RGAQVFPS1-06 }\end{array}$ & NM & Bernalillo & $7 / 14 / 2016$ & 4,980 & NGVD 29 & Observation & 34.8 & 17.5 & 32.5 \\
\hline $\begin{array}{l}\text { Rio Grande aqui- } \\
\text { fer system }\end{array}$ & $\begin{array}{l}\text { LUS/ } \\
\text { VFPS }\end{array}$ & $\begin{array}{l}\text { rioglusrc1/ } \\
\text { rgaqvfps1 }\end{array}$ & $\begin{array}{l}\text { RIOGLUSRC1-10/ } \\
\text { RGAQVFPS1-07 }\end{array}$ & $\mathrm{NM}$ & Bernalillo & $7 / 14 / 2016$ & $4,969.80$ & NGVD 29 & Observation & 39.7 & 30 & 35 \\
\hline
\end{tabular}


Table 1. Information about wells that have environmental data included in this report.-Continued

[NAWQA, National Water-Quality Assessment; ID, identification; no., number; LS, land surface; $\mathrm{ft}$ bls, foot below land surface; lat., latitude, in degrees and minutes; long., longitude in degrees and minutes; PAS, principal aquifer study; FL, Florida; na, not available; NGVD 29, National Geodetic Vertical Datum of 1929; NAVD 88, North American Vertical Datum of 1988; ETN, enhanced trends network; TX,

Texas; NM, New Mexico; LUS, land-use study; SC, South Carolina; FPS, flow-path study; MS, Mississippi; VFPS; vertical flow-path study; TN, Tennessee; OK, Oklahoma; AR, Arkansas; CA, California; MO, Missouri; KS, Kansas; MD, Maryland; MSS, modeling support study; DE, Delaware; DC, District of Columbia; MAS, major aquifer study; NV, Nevada; NY, New York; OH, Ohio; MI, Michigan; ID, Idaho;

NH, New Hampshire; WI, Wisconsin; OR, Oregon; MN, Minnesota; WA, Washington]

\begin{tabular}{|c|c|c|c|c|c|c|c|c|c|c|c|c|}
\hline \multirow{2}{*}{$\begin{array}{l}\text { Principal and } \\
\text { regional and (or) } \\
\text { other aquifer } \\
\text { information }\end{array}$} & \multirow{2}{*}{$\begin{array}{l}\text { Network } \\
\text { type }\end{array}$} & \multirow{2}{*}{$\begin{array}{l}\text { Network } \\
\text { name }\end{array}$} & \multirow{2}{*}{$\begin{array}{l}\text { NAWQA well } \\
\text { ID no. }\end{array}$} & \multirow{2}{*}{ State } & \multirow{2}{*}{ County } & \multirow{2}{*}{ Sample date } & \multirow{2}{*}{$\begin{array}{l}\text { Altitude } \\
\text { LS }\end{array}$} & \multirow{2}{*}{$\begin{array}{l}\text { Altitude } \\
\text { datum }\end{array}$} & \multirow{2}{*}{ Water use } & \multirow{2}{*}{$\begin{array}{c}\text { Well } \\
\text { depth } \\
\text { (ft bls) }\end{array}$} & \multicolumn{2}{|c|}{$\begin{array}{c}\text { Depth to perforation } \\
\text { (ft bls) }\end{array}$} \\
\hline & & & & & & & & & & & Top & Bottom \\
\hline $\begin{array}{l}\text { Rio Grande aqui- } \\
\text { fer system }\end{array}$ & $\begin{array}{l}\text { LUS/ } \\
\text { VFPS }\end{array}$ & $\begin{array}{l}\text { rioglusrc1/ } \\
\text { rgaqvfps1 }\end{array}$ & $\begin{array}{l}\text { RIOGLUSRC1-11/ } \\
\text { RGAQVFPS1-08 }\end{array}$ & NM & Bernalillo & $7 / 14 / 2016$ & $4,972.30$ & NGVD 29 & Observation & 49.8 & 40 & 45 \\
\hline $\begin{array}{l}\text { Rio Grande aqui- } \\
\text { fer system }\end{array}$ & LUS & rioglusrc1 & RIOGLUSRC1-12 & $\mathrm{NM}$ & Valencia & $7 / 18 / 2016$ & 4,800 & NGVD 29 & Observation & 29.5 & 16.5 & 26.5 \\
\hline $\begin{array}{l}\text { Rio Grande aqui- } \\
\text { fer system }\end{array}$ & LUS & rioglusrc1 & RIOGLUSRC1-13 & $\mathrm{NM}$ & Bernalillo & $7 / 18 / 2016$ & 5,002 & NGVD 29 & Observation & 22.9 & 11 & 21 \\
\hline $\begin{array}{l}\text { Rio Grande aqui- } \\
\text { fer system }\end{array}$ & LUS & rioglusrc1 & RIOGLUSRC1-14 & $\mathrm{NM}$ & Valencia & $7 / 18 / 2016$ & 4,852 & NGVD 29 & Observation & 20.4 & 5 & 15 \\
\hline $\begin{array}{l}\text { Rio Grande aqui- } \\
\text { fer system }\end{array}$ & LUS & rioglusrc1 & RIOGLUSRC1-15 & $\mathrm{NM}$ & Valencia & $7 / 19 / 2016$ & 4,848 & NGVD 29 & Observation & 21.2 & 7 & 17 \\
\hline $\begin{array}{l}\text { Rio Grande aqui- } \\
\text { fer system }\end{array}$ & LUS & rioglusrc1 & RIOGLUSRC1-16 & $\mathrm{NM}$ & Bernalillo & $7 / 19 / 2016$ & 4,925 & NGVD 29 & Observation & 18.9 & 8.3 & 18.3 \\
\hline $\begin{array}{l}\text { Rio Grande aqui- } \\
\text { fer system }\end{array}$ & LUS & rioglusrc1 & RIOGLUSRC1-17 & $\mathrm{NM}$ & Bernalillo & $7 / 19 / 2016$ & 4,919 & NGVD 29 & Observation & 22.1 & 9.5 & 19.5 \\
\hline $\begin{array}{l}\text { Rio Grande aqui- } \\
\text { fer system }\end{array}$ & $\begin{array}{l}\text { LUS/ } \\
\text { VFPS }\end{array}$ & $\begin{array}{l}\text { rioglusrc1/ } \\
\text { rgaqvfps1 }\end{array}$ & $\begin{array}{l}\text { RIOGLUSRC1-18/ } \\
\text { RGAQVFPS1-17 }\end{array}$ & NM & Bernalillo & $7 / 19 / 2016$ & 4,935 & NGVD 29 & Observation & 28.3 & 18 & 28 \\
\hline $\begin{array}{l}\text { Rio Grande aqui- } \\
\text { fer system }\end{array}$ & LUS & rioglusrc1 & RIOGLUSRC1-19 & $\mathrm{NM}$ & Valencia & $7 / 20 / 2016$ & 4,845 & NGVD 29 & Observation & 22.3 & 8.5 & 18.5 \\
\hline $\begin{array}{l}\text { Rio Grande aqui- } \\
\text { fer system }\end{array}$ & $\begin{array}{l}\text { LUS/ } \\
\text { VFPS }\end{array}$ & $\begin{array}{l}\text { rioglusrc1/ } \\
\text { rgaqvfps1 }\end{array}$ & $\begin{array}{l}\text { RIOGLUSRC1-20/ } \\
\text { RGAQVFPS1-05 }\end{array}$ & $\mathrm{NM}$ & Bernalillo & $7 / 20 / 2016$ & 4,989 & NGVD 29 & Observation & 57 & 41 & 56 \\
\hline $\begin{array}{l}\text { Rio Grande aqui- } \\
\text { fer system }\end{array}$ & LUS & rioglusrc1 & RIOGLUSRC1-21 & $\mathrm{NM}$ & Bernalillo & $7 / 20 / 2016$ & 4,920 & NGVD 29 & Observation & 19.9 & 7.5 & 17.5 \\
\hline $\begin{array}{l}\text { Rio Grande aqui- } \\
\text { fer system }\end{array}$ & LUS & rioglusrc1 & RIOGLUSRC1-22 & $\mathrm{NM}$ & Sandoval & $7 / 20 / 2016$ & 5,053 & NGVD 29 & Observation & 28.1 & 18.1 & 27.7 \\
\hline $\begin{array}{l}\text { Rio Grande aqui- } \\
\text { fer system }\end{array}$ & LUS & rioglusrc1 & RIOGLUSRC1-23 & $\mathrm{NM}$ & Bernalillo & $7 / 21 / 2016$ & 4,925 & NGVD 29 & Observation & 12.5 & 7 & 12 \\
\hline $\begin{array}{l}\text { Rio Grande aqui- } \\
\text { fer system }\end{array}$ & $\begin{array}{l}\text { LUS/ } \\
\text { VFPS }\end{array}$ & $\begin{array}{l}\text { rioglusrc1/ } \\
\text { rgaqvfps1 }\end{array}$ & $\begin{array}{l}\text { RIOGLUSRC1-24/ } \\
\text { RGAQVFPS1-01 }\end{array}$ & $\mathrm{NM}$ & Bernalillo & $7 / 21 / 2016$ & 4,995 & NGVD 29 & Observation & 31.5 & 21.4 & 31.4 \\
\hline
\end{tabular}


Table 1. Information about wells that have environmental data included in this report.-Continued

[NAWQA, National Water-Quality Assessment; ID, identification; no., number; LS, land surface; ft bls, foot below land surface; lat., latitude, in degrees and minutes; long., longitude in degrees and minutes; PAS, principal aquifer study; FL, Florida; na, not available; NGVD 29, National Geodetic Vertical Datum of 1929; NAVD 88, North American Vertical Datum of 1988; ETN, enhanced trends network; TX, Texas; NM, New Mexico; LUS, land-use study; SC, South Carolina; FPS, flow-path study; MS, Mississippi; VFPS; vertical flow-path study; TN, Tennessee; OK, Oklahoma; AR, Arkansas; CA, California; MO, Missouri; KS, Kansas; MD, Maryland; MSS, modeling support study; DE, Delaware; DC, District of Columbia; MAS, major aquifer study; NV, Nevada; NY, New York; OH, Ohio; MI, Michigan; ID, Idaho; NH, New Hampshire; WI, Wisconsin; OR, Oregon; MN, Minnesota; WA, Washington]

\begin{tabular}{|c|c|c|c|c|c|c|c|c|c|c|c|c|}
\hline \multirow{2}{*}{$\begin{array}{l}\text { Principal and } \\
\text { regional and (or) } \\
\text { other aquifer } \\
\text { information }\end{array}$} & \multirow{2}{*}{$\begin{array}{l}\text { Network } \\
\text { type }\end{array}$} & \multirow{2}{*}{$\begin{array}{l}\text { Network } \\
\text { name }\end{array}$} & \multirow{2}{*}{$\begin{array}{l}\text { NAWQA well } \\
\text { ID no. }\end{array}$} & \multirow{2}{*}{ State } & \multirow{2}{*}{ County } & \multirow{2}{*}{ Sample date } & \multirow{2}{*}{$\begin{array}{c}\text { Altitude } \\
\text { LS }\end{array}$} & \multirow{2}{*}{$\begin{array}{l}\text { Altitude } \\
\text { datum }\end{array}$} & \multirow{2}{*}{ Water use } & \multirow{2}{*}{$\begin{array}{r}\text { Well } \\
\text { depth } \\
\text { (ft bls) }\end{array}$} & \multicolumn{2}{|c|}{$\begin{array}{c}\text { Depth to perforation } \\
\text { (ft bls) }\end{array}$} \\
\hline & & & & & & & & & & & Top & Bottom \\
\hline $\begin{array}{l}\text { Rio Grande aqui- } \\
\text { fer system }\end{array}$ & LUS & rioglusrc1 & RIOGLUSRC1-25 & NM & Sandoval & $8 / 1 / 2016$ & 5,015 & NGVD 29 & Observation & 29 & 18 & 28 \\
\hline $\begin{array}{l}\text { Rio Grande aqui- } \\
\text { fer system }\end{array}$ & $\begin{array}{l}\text { LUS/ } \\
\text { VFPS }\end{array}$ & $\begin{array}{l}\text { rioglusrc1/ } \\
\text { rgaqvfps1 }\end{array}$ & $\begin{array}{l}\text { RIOGLUSRC1-26/ } \\
\text { RGAQVFPS1-10 }\end{array}$ & $\mathrm{NM}$ & Bernalillo & $8 / 4 / 2016$ & 4,965 & NGVD 29 & Observation & 27.8 & 17.5 & 27.5 \\
\hline $\begin{array}{l}\text { Rio Grande aqui- } \\
\text { fer system }\end{array}$ & LUS & rioglusrc1 & RIOGLUSRC1-27 & NM & Bernalillo & $8 / 8 / 2016$ & 4,865 & NGVD 29 & Observation & 24.3 & 11.5 & 21.5 \\
\hline $\begin{array}{l}\text { Rio Grande aqui- } \\
\text { fer system }\end{array}$ & VFPS & rgaqvfps 1 & RGAQVFPS1-03 & NM & Bernalillo & $7 / 22 / 2016$ & $4,988.60$ & NGVD 29 & Observation & 95 & 8 & 90 \\
\hline $\begin{array}{l}\text { Rio Grande aqui- } \\
\text { fer system }\end{array}$ & VFPS & rgaqvfps1 & RGAQVFPS1-09 & NM & Bernalillo & $7 / 26 / 2016$ & $4,972.30$ & NGVD 29 & Observation & 149.8 & 140 & 145 \\
\hline $\begin{array}{l}\text { Rio Grande aqui- } \\
\text { fer system }\end{array}$ & VFPS & rgaqvfps1 & RGAQVFPS1-12 & NM & Bernalillo & $7 / 25 / 2016$ & 5,110 & NAVD 88 & Observation & 254 & 244 & 249 \\
\hline $\begin{array}{l}\text { Rio Grande aqui- } \\
\text { fer system }\end{array}$ & VFPS & rgaqvfps 1 & RGAQVFPS1-14 & NM & Bernalillo & $7 / 21 / 2016$ & $4,961.70$ & NGVD 29 & Observation & 100 & 95 & 100 \\
\hline $\begin{array}{l}\text { Snake River } \\
\text { Plain aquifer } \\
\text { system }\end{array}$ & LUS & usnkluscr2 & USNKLUSCR2-01 & ID & Minidoka & $6 / 13 / 2016$ & $4,151.40$ & NAVD 88 & Domestic & 150 & 113 & 150 \\
\hline $\begin{array}{l}\text { Snake River } \\
\text { Plain aquifer } \\
\text { system }\end{array}$ & LUS & usnkluscr2 & USNKLUSCR2-02 & ID & Minidoka & $6 / 13 / 2016$ & $4,191.50$ & NAVD 88 & Domestic & 190 & 19 & 190 \\
\hline $\begin{array}{l}\text { Snake River } \\
\text { Plain aquifer } \\
\text { system }\end{array}$ & LUS & usnkluscr2 & USNKLUSCR2-03 & ID & Minidoka & $6 / 14 / 2016$ & $4,151.60$ & NAVD 88 & Domestic & 130 & 127 & 129 \\
\hline $\begin{array}{l}\text { Snake River } \\
\text { Plain aquifer } \\
\text { system }\end{array}$ & LUS & usnkluscr2 & USNKLUSCR2-04 & ID & Minidoka & $6 / 14 / 2016$ & $4,216.10$ & NAVD 88 & Domestic & 265 & 167 & 265 \\
\hline $\begin{array}{l}\text { Snake River } \\
\text { Plain aquifer } \\
\text { system }\end{array}$ & LUS & usnkluscr2 & USNKLUSCR2-05 & ID & Minidoka & $6 / 14 / 2016$ & $4,157.20$ & NAVD 88 & Domestic & 215 & 161 & 215 \\
\hline
\end{tabular}


Table 1. Information about wells that have environmental data included in this report.-Continued

[NAWQA, National Water-Quality Assessment; ID, identification; no., number; LS, land surface; ft bls, foot below land surface; lat., latitude, in degrees and minutes; long., longitude in degrees and minutes; PAS, principal aquifer study; FL, Florida; na, not available; NGVD 29, National Geodetic Vertical Datum of 1929; NAVD 88, North American Vertical Datum of 1988; ETN, enhanced trends network; TX,

Texas; NM, New Mexico; LUS, land-use study; SC, South Carolina; FPS, flow-path study; MS, Mississippi; VFPS; vertical flow-path study; TN, Tennessee; OK, Oklahoma; AR, Arkansas; CA, California; MO, Missouri; KS, Kansas; MD, Maryland; MSS, modeling support study; DE, Delaware; DC, District of Columbia; MAS, major aquifer study; NV, Nevada; NY, New York; OH, Ohio; MI, Michigan; ID, Idaho;

NH, New Hampshire; WI, Wisconsin; OR, Oregon; MN, Minnesota; WA, Washington]

\begin{tabular}{|c|c|c|c|c|c|c|c|c|c|c|c|c|}
\hline \multirow{2}{*}{$\begin{array}{l}\text { Principal and } \\
\text { regional and (or) } \\
\text { other aquifer } \\
\text { information }\end{array}$} & \multirow{2}{*}{$\begin{array}{c}\text { Network } \\
\text { type }\end{array}$} & \multirow{2}{*}{$\begin{array}{l}\text { Network } \\
\text { name }\end{array}$} & \multirow{2}{*}{$\begin{array}{l}\text { NAWQA well } \\
\text { ID no. }\end{array}$} & \multirow{2}{*}{ State } & \multirow{2}{*}{ County } & \multirow{2}{*}{ Sample date } & \multirow{2}{*}{$\begin{array}{c}\text { Altitude } \\
\text { LS }\end{array}$} & \multirow{2}{*}{$\begin{array}{l}\text { Altitude } \\
\text { datum }\end{array}$} & \multirow{2}{*}{ Water use } & \multirow{2}{*}{$\begin{array}{c}\text { Well } \\
\text { depth } \\
\text { (ft bls) }\end{array}$} & \multicolumn{2}{|c|}{$\begin{array}{c}\text { Depth to perforation } \\
\text { (ft bls) }\end{array}$} \\
\hline & & & & & & & & & & & Top & Bottom \\
\hline $\begin{array}{l}\text { Snake River } \\
\quad \text { Plain aquifer } \\
\text { system }\end{array}$ & LUS & usnkluscr2 & USNKLUSCR2-06 & ID & Blaine & $6 / 15 / 2016$ & $4,338.30$ & NAVD 88 & Domestic & 282 & 147 & 282 \\
\hline $\begin{array}{l}\text { Snake River } \\
\text { Plain aquifer } \\
\text { system }\end{array}$ & LUS & usnkluscr2 & USNKLUSCR2-07 & ID & Minidoka & $6 / 15 / 2016$ & $4,279.80$ & NAVD 88 & Domestic & 225 & 8 & 225 \\
\hline $\begin{array}{l}\text { Snake River } \\
\text { Plain aquifer } \\
\text { system }\end{array}$ & LUS & usnkluscr2 & USNKLUSCR2-08 & ID & Minidoka & $6 / 15 / 2016$ & 4,305 & NAVD 88 & Domestic & 275 & 19 & 275 \\
\hline $\begin{array}{l}\text { Snake River } \\
\text { Plain aquifer } \\
\text { system }\end{array}$ & LUS & usnkluscr2 & USNKLUSCR2-09 & ID & Minidoka & $6 / 15 / 2016$ & $4,244.20$ & NAVD 88 & Domestic & 255 & 38 & 255 \\
\hline $\begin{array}{l}\text { Snake River } \\
\text { Plain aquifer } \\
\text { system }\end{array}$ & LUS & usnkluscr2 & USNKLUSCR2-10 & ID & Minidoka & $6 / 16 / 2016$ & $4,344.60$ & NAVD 88 & Domestic & 469 & na & $469 \mathrm{a}$ \\
\hline $\begin{array}{l}\text { Snake River } \\
\text { Plain aquifer } \\
\text { system }\end{array}$ & LUS & usnkluscr2 & USNKLUSCR2-11 & ID & Minidoka & $6 / 16 / 2016$ & $4,181.20$ & NAVD 88 & Domestic & 180 & 119 & 180 \\
\hline $\begin{array}{l}\text { Snake River } \\
\text { Plain aquifer } \\
\text { system }\end{array}$ & LUS & usnkluscr2 & USNKLUSCR2-12 & ID & Minidoka & $6 / 20 / 2016$ & $4,299.60$ & NAVD 88 & Domestic & 331 & na & $331^{a}$ \\
\hline $\begin{array}{l}\text { Snake River } \\
\quad \text { Plain aquifer } \\
\text { system }\end{array}$ & LUS & usnkluscr2 & USNKLUSCR2-13 & ID & Minidoka & $6 / 20 / 2016$ & 4,153 & NAVD 88 & Domestic & 125 & 109 & 114 \\
\hline $\begin{array}{l}\text { Snake River } \\
\text { Plain aquifer } \\
\text { system }\end{array}$ & LUS & usnkluscr2 & USNKLUSCR2-14 & ID & Minidoka & $6 / 21 / 2016$ & $4,302.40$ & NGVD 29 & Domestic & 350 & 16 & 350 \\
\hline $\begin{array}{l}\text { Snake River } \\
\text { Plain aquifer } \\
\text { system }\end{array}$ & LUS & usnkluscr2 & USNKLUSCR2-15 & ID & Lincoln & $6 / 21 / 2016$ & $4,289.50$ & NAVD 88 & Domestic & 313 & na & $313^{a}$ \\
\hline
\end{tabular}


Table 1. Information about wells that have environmental data included in this report.-Continued

[NAWQA, National Water-Quality Assessment; ID, identification; no., number; LS, land surface; ft bls, foot below land surface; lat., latitude, in degrees and minutes; long., longitude in degrees and minutes; PAS, principal aquifer study; FL, Florida; na, not available; NGVD 29, National Geodetic Vertical Datum of 1929; NAVD 88, North American Vertical Datum of 1988; ETN, enhanced trends network; TX, Texas; NM, New Mexico; LUS, land-use study; SC, South Carolina; FPS, flow-path study; MS, Mississippi; VFPS; vertical flow-path study; TN, Tennessee; OK, Oklahoma; AR, Arkansas; CA, California; MO, Missouri; KS, Kansas; MD, Maryland; MSS, modeling support study; DE, Delaware; DC, District of Columbia; MAS, major aquifer study; NV, Nevada; NY, New York; OH, Ohio; MI, Michigan; ID, Idaho; NH, New Hampshire; WI, Wisconsin; OR, Oregon; MN, Minnesota; WA, Washington]

\begin{tabular}{|c|c|c|c|c|c|c|c|c|c|c|c|c|}
\hline \multirow{2}{*}{$\begin{array}{l}\text { Principal and } \\
\text { regional and (or) } \\
\text { other aquifer } \\
\text { information }\end{array}$} & \multirow{2}{*}{$\begin{array}{l}\text { Network } \\
\text { type }\end{array}$} & \multirow{2}{*}{$\begin{array}{l}\text { Network } \\
\text { name }\end{array}$} & \multirow{2}{*}{$\begin{array}{l}\text { NAWQA well } \\
\text { ID no. }\end{array}$} & \multirow{2}{*}{ State } & \multirow{2}{*}{ County } & \multirow{2}{*}{ Sample date } & \multirow{2}{*}{$\begin{array}{l}\text { Altitude } \\
\text { LS }\end{array}$} & \multirow{2}{*}{$\begin{array}{l}\text { Altitude } \\
\text { datum }\end{array}$} & \multirow{2}{*}{ Water use } & \multirow{2}{*}{$\begin{array}{c}\text { Well } \\
\text { depth } \\
\text { (ft bls) }\end{array}$} & \multicolumn{2}{|c|}{$\begin{array}{c}\text { Depth to perforation } \\
\text { (ft bls) }\end{array}$} \\
\hline & & & & & & & & & & & Top & Bottom \\
\hline $\begin{array}{l}\text { Snake River } \\
\text { Plain aquifer } \\
\text { system }\end{array}$ & LUS & usnkluscr2 & USNKLUSCR2-16 & ID & Lincoln & $6 / 21 / 2016$ & $4,292.10$ & NGVD 29 & Other & 355 & 18.5 & 355 \\
\hline $\begin{array}{l}\text { Snake River } \\
\quad \text { Plain aquifer } \\
\text { system }\end{array}$ & LUS & usnkluscr2 & USNKLUSCR2-17 & ID & Minidoka & $6 / 21 / 2016$ & $4,304.10$ & NAVD 88 & Domestic & na & na & na \\
\hline $\begin{array}{l}\text { Snake River } \\
\text { Plain aquifer } \\
\text { system }\end{array}$ & LUS & usnkluscr2 & USNKLUSCR2-18 & ID & Minidoka & $6 / 22 / 2016$ & 4,193 & NAVD 88 & Domestic & 180 & 20 & 180 \\
\hline $\begin{array}{l}\text { Snake River } \\
\quad \text { Plain aquifer } \\
\text { system }\end{array}$ & LUS & usnkluscr2 & USNKLUSCR2-19 & ID & Minidoka & $6 / 22 / 2016$ & $4,170.10$ & NAVD 88 & Domestic & 165 & 24 & 165 \\
\hline $\begin{array}{l}\text { Snake River } \\
\text { Plain aquifer } \\
\text { system }\end{array}$ & LUS & usnkluscr2 & USNKLUSCR2-20 & ID & Minidoka & $6 / 22 / 2016$ & 4,253 & NAVD 88 & Domestic & 280 & 18 & 280 \\
\hline $\begin{array}{l}\text { Snake River } \\
\quad \text { Plain aquifer } \\
\text { system }\end{array}$ & LUS & usnkluscr2 & USNKLUSCR2-21 & ID & Minidoka & $6 / 22 / 2016$ & $4,209.10$ & NAVD 88 & Domestic & 197 & 19 & 197 \\
\hline $\begin{array}{l}\text { Snake River } \\
\text { Plain aquifer } \\
\text { system }\end{array}$ & LUS & usnkluscr2 & USNKLUSCR2-22 & ID & Minidoka & $6 / 23 / 2016$ & 4,303 & NAVD 88 & Domestic & 365 & 19 & 275 \\
\hline $\begin{array}{l}\text { Snake River } \\
\text { Plain aquifer } \\
\text { system }\end{array}$ & LUS & usnkluscr2 & USNKLUSCR2-23 & ID & Minidoka & $6 / 27 / 2016$ & $4,260.80$ & NAVD 88 & Domestic & 283 & na & $283^{a}$ \\
\hline $\begin{array}{l}\text { Snake River } \\
\text { Plain aquifer } \\
\text { system }\end{array}$ & LUS & usnkluscr2 & USNKLUSCR2-24 & ID & Minidoka & $6 / 27 / 2016$ & $4,278.70$ & NAVD 88 & Domestic & 300 & 30 & 300 \\
\hline $\begin{array}{l}\text { Snake River } \\
\quad \text { Plain aquifer } \\
\text { system }\end{array}$ & LUS & usnkluscr2 & USNKLUSCR2-25 & ID & Minidoka & $6 / 27 / 2016$ & $4,249.80$ & NAVD 88 & Domestic & 284 & 6 & 284 \\
\hline
\end{tabular}


Table 1. Information about wells that have environmental data included in this report.-Continued

[NAWQA, National Water-Quality Assessment; ID, identification; no., number; LS, land surface; ft bls, foot below land surface; lat., latitude, in degrees and minutes; long., longitude in degrees and minutes; PAS, principal aquifer study; FL, Florida; na, not available; NGVD 29, National Geodetic Vertical Datum of 1929; NAVD 88, North American Vertical Datum of 1988; ETN, enhanced trends network; TX,

Texas; NM, New Mexico; LUS, land-use study; SC, South Carolina; FPS, flow-path study; MS, Mississippi; VFPS; vertical flow-path study; TN, Tennessee; OK, Oklahoma; AR, Arkansas; CA, California; MO, Missouri; KS, Kansas; MD, Maryland; MSS, modeling support study; DE, Delaware; DC, District of Columbia; MAS, major aquifer study; NV, Nevada; NY, New York; OH, Ohio; MI, Michigan; ID, Idaho;

NH, New Hampshire; WI, Wisconsin; OR, Oregon; MN, Minnesota; WA, Washington]

\begin{tabular}{|c|c|c|c|c|c|c|c|c|c|c|c|c|}
\hline \multirow{2}{*}{$\begin{array}{l}\text { Principal and } \\
\text { regional and (or) } \\
\text { other aquifer } \\
\text { information }\end{array}$} & \multirow{2}{*}{$\begin{array}{l}\text { Network } \\
\text { type }\end{array}$} & \multirow{2}{*}{$\begin{array}{l}\text { Network } \\
\text { name }\end{array}$} & \multirow{2}{*}{$\begin{array}{l}\text { NAWQA well } \\
\text { ID no. }\end{array}$} & \multirow{2}{*}{ State } & \multirow{2}{*}{ County } & \multirow{2}{*}{ Sample date } & \multirow{2}{*}{$\begin{array}{l}\text { Altitude } \\
\text { LS }\end{array}$} & \multirow{2}{*}{$\begin{array}{l}\text { Altitude } \\
\text { datum }\end{array}$} & \multirow{2}{*}{ Water use } & \multirow{2}{*}{$\begin{array}{c}\text { Well } \\
\text { depth } \\
\text { (ft bls) }\end{array}$} & \multicolumn{2}{|c|}{$\begin{array}{c}\text { Depth to perforation } \\
\text { (ft bls) }\end{array}$} \\
\hline & & & & & & & & & & & Top & Bottom \\
\hline $\begin{array}{l}\text { Snake River } \\
\text { Plain aquifer } \\
\text { system }\end{array}$ & LUS & usnkluscr2 & USNKLUSCR2-26 & ID & Minidoka & $6 / 28 / 2016$ & $4,210.10$ & NAVD 88 & Domestic & 250 & 198 & 250 \\
\hline $\begin{array}{l}\text { Snake River } \\
\text { Plain aquifer } \\
\text { system }\end{array}$ & LUS & usnkluscr2 & USNKLUSCR2-27 & ID & Minidoka & $6 / 28 / 2016$ & $4,201.20$ & NAVD 88 & Domestic & 200 & na & $200^{a}$ \\
\hline $\begin{array}{l}\text { Snake River } \\
\text { Plain aquifer } \\
\text { system }\end{array}$ & LUS & usnkluscr2 & USNKLUSCR2-28 & ID & Minidoka & $6 / 28 / 2016$ & $4,210.20$ & NAVD 88 & Domestic & 214 & 89 & 147 \\
\hline $\begin{array}{l}\text { Snake River } \\
\text { Plain aquifer } \\
\text { system }\end{array}$ & LUS & usnkluscr2 & USNKLUSCR2-29 & ID & Minidoka & $6 / 29 / 2016$ & $4,223.60$ & NAVD 88 & Domestic & 260 & 55 & 260 \\
\hline $\begin{array}{l}\text { Snake River } \\
\text { Plain aquifer } \\
\text { system }\end{array}$ & LUS & usnkluscr2 & USNKLUSCR2-30 & ID & Minidoka & $6 / 29 / 2016$ & $4,239.30$ & NAVD 88 & Domestic & 257 & 15 & 257 \\
\hline $\begin{array}{l}\text { Snake River } \\
\text { Plain aquifer } \\
\text { system }\end{array}$ & LUS & usnkluscr2 & USNKLUSCR2-31 & ID & Minidoka & $6 / 29 / 2016$ & 4,211 & NAVD 88 & Domestic & 200 & 165 & 200 \\
\hline $\begin{array}{l}\text { Southeastern } \\
\text { Coastal Plain } \\
\text { aquifer system }\end{array}$ & LUS & santlusrc1 & SANTLUSRC1-01 & $\mathrm{SC}$ & Richland & $8 / 1 / 2016$ & 244 & NAVD 88 & Observation & 49 & 34 & 49 \\
\hline $\begin{array}{l}\text { Southeastern } \\
\text { Coastal Plain } \\
\text { aquifer system }\end{array}$ & LUS & santlusrc1 & SANTLUSRC1-02 & $\mathrm{SC}$ & Richland & $8 / 2 / 2016$ & 219 & NGVD 29 & Observation & 19.5 & 14.4 & 19.4 \\
\hline $\begin{array}{l}\text { Southeastern } \\
\text { Coastal Plain } \\
\text { aquifer system }\end{array}$ & LUS & santlusrc1 & SANTLUSRC1-03 & $\mathrm{SC}$ & Richland & $8 / 3 / 2016$ & 261 & NGVD 29 & Observation & 47 & 42 & 47 \\
\hline $\begin{array}{l}\text { Southeastern } \\
\text { Coastal Plain } \\
\text { aquifer system }\end{array}$ & LUS & santlusrc1 & SANTLUSRC1-04 & $\mathrm{SC}$ & Richland & $8 / 3 / 2016$ & 260 & NGVD 29 & Observation & 21.5 & 16.5 & 21.5 \\
\hline
\end{tabular}


Table 1. Information about wells that have environmental data included in this report.-Continued

[NAWQA, National Water-Quality Assessment; ID, identification; no., number; LS, land surface; ft bls, foot below land surface; lat., latitude, in degrees and minutes; long., longitude in degrees and minutes; PAS, principal aquifer study; FL, Florida; na, not available; NGVD 29, National Geodetic Vertical Datum of 1929; NAVD 88, North American Vertical Datum of 1988; ETN, enhanced trends network; TX, Texas; NM, New Mexico; LUS, land-use study; SC, South Carolina; FPS, flow-path study; MS, Mississippi; VFPS; vertical flow-path study; TN, Tennessee; OK, Oklahoma; AR, Arkansas; CA, California; MO, Missouri; KS, Kansas; MD, Maryland; MSS, modeling support study; DE, Delaware; DC, District of Columbia; MAS, major aquifer study; NV, Nevada; NY, New York; OH, Ohio; MI, Michigan; ID, Idaho; NH, New Hampshire; WI, Wisconsin; OR, Oregon; MN, Minnesota; WA, Washington]

\begin{tabular}{|c|c|c|c|c|c|c|c|c|c|c|c|c|}
\hline \multirow{2}{*}{$\begin{array}{l}\text { Principal and } \\
\text { regional and (or) } \\
\text { other aquifer } \\
\text { information }\end{array}$} & \multirow{2}{*}{$\begin{array}{l}\text { Network } \\
\text { type }\end{array}$} & \multirow{2}{*}{$\begin{array}{l}\text { Network } \\
\text { name }\end{array}$} & \multirow{2}{*}{$\begin{array}{l}\text { NAWQA well } \\
\text { ID no. }\end{array}$} & \multirow{2}{*}{ State } & \multirow{2}{*}{ County } & \multirow{2}{*}{ Sample date } & \multirow{2}{*}{$\begin{array}{l}\text { Altitude } \\
\text { LS }\end{array}$} & \multirow{2}{*}{$\begin{array}{l}\text { Altitude } \\
\text { datum }\end{array}$} & \multirow{2}{*}{ Water use } & \multirow{2}{*}{$\begin{array}{c}\text { Well } \\
\text { depth } \\
\text { (ft bls) }\end{array}$} & \multicolumn{2}{|c|}{$\begin{array}{c}\text { Depth to perforation } \\
\text { (ft bls) }\end{array}$} \\
\hline & & & & & & & & & & & Top & Bottom \\
\hline $\begin{array}{l}\text { Southeastern } \\
\text { Coastal Plain } \\
\text { aquifer system }\end{array}$ & LUS & santlusrc1 & SANTLUSRC1-05 & $\mathrm{SC}$ & Richland & $8 / 4 / 2016$ & 250 & NGVD 29 & Observation & 13.5 & 8.5 & 13.5 \\
\hline $\begin{array}{l}\text { Southeastern } \\
\text { Coastal Plain } \\
\text { aquifer system }\end{array}$ & LUS & santlusrc1 & SANTLUSRC1-06 & $\mathrm{SC}$ & Richland & $8 / 8 / 2016$ & 290 & NGVD 29 & Observation & 24.2 & 19.2 & 24.2 \\
\hline $\begin{array}{l}\text { Southeastern } \\
\text { Coastal Plain } \\
\text { aquifer system }\end{array}$ & LUS & santlusrc1 & SANTLUSRC1-07 & $\mathrm{SC}$ & Richland & $8 / 9 / 2016$ & 400 & NGVD 29 & Observation & 54 & 49 & 54 \\
\hline $\begin{array}{l}\text { Southeastern } \\
\text { Coastal Plain } \\
\text { aquifer system }\end{array}$ & LUS & santlusrc1 & SANTLUSRC1-08 & $\mathrm{SC}$ & Richland & $8 / 15 / 2016$ & 320 & NGVD 29 & Observation & 24.3 & 19.3 & 24.3 \\
\hline $\begin{array}{l}\text { Southeastern } \\
\text { Coastal Plain } \\
\text { aquifer system }\end{array}$ & LUS & santlusrc1 & SANTLUSRC1-09 & $\mathrm{SC}$ & Richland & $8 / 15 / 2016$ & 380 & NGVD 29 & Observation & 15.9 & 10.9 & 15.9 \\
\hline $\begin{array}{l}\text { Southeastern } \\
\text { Coastal Plain } \\
\text { aquifer system }\end{array}$ & LUS & santlusrc1 & SANTLUSRC1-10 & $\mathrm{SC}$ & Richland & $8 / 16 / 2016$ & 215 & NGVD 29 & Observation & 11.1 & 6.1 & 11.1 \\
\hline $\begin{array}{l}\text { Southeastern } \\
\text { Coastal Plain } \\
\text { aquifer system }\end{array}$ & LUS & santlusrc1 & SANTLUSRC1-11 & $\mathrm{SC}$ & Richland & $8 / 16 / 2016$ & 240 & NGVD 29 & Observation & 33.3 & 28 & 33 \\
\hline $\begin{array}{l}\text { Southeastern } \\
\text { Coastal Plain } \\
\text { aquifer system }\end{array}$ & LUS & santlusrc1 & SANTLUSRC1-12 & $\mathrm{SC}$ & Richland & $8 / 16 / 2016$ & 293 & NGVD 29 & Observation & 50.4 & 45.4 & 50.4 \\
\hline $\begin{array}{l}\text { Southeastern } \\
\text { Coastal Plain } \\
\text { aquifer system }\end{array}$ & LUS & santlusrc1 & SANTLUSRC1-13 & $\mathrm{SC}$ & Richland & $8 / 17 / 2016$ & 230 & NGVD 29 & Observation & 11.2 & 6.1 & 11.2 \\
\hline $\begin{array}{l}\text { Southeastern } \\
\text { Coastal Plain } \\
\text { aquifer system }\end{array}$ & LUS & santlusrc1 & SANTLUSRC1-14 & $\mathrm{SC}$ & Richland & $8 / 17 / 2016$ & 210 & NGVD 29 & Observation & 15.4 & 10.4 & 15.4 \\
\hline
\end{tabular}


Table 1. Information about wells that have environmental data included in this report.-Continued

[NAWQA, National Water-Quality Assessment; ID, identification; no., number; LS, land surface; ft bls, foot below land surface; lat., latitude, in degrees and minutes; long., longitude in degrees and minutes; PAS, principal aquifer study; FL, Florida; na, not available; NGVD 29, National Geodetic Vertical Datum of 1929; NAVD 88, North American Vertical Datum of 1988; ETN, enhanced trends network; TX,

Texas; NM, New Mexico; LUS, land-use study; SC, South Carolina; FPS, flow-path study; MS, Mississippi; VFPS; vertical flow-path study; TN, Tennessee; OK, Oklahoma; AR, Arkansas; CA, California; MO, Missouri; KS, Kansas; MD, Maryland; MSS, modeling support study; DE, Delaware; DC, District of Columbia; MAS, major aquifer study; NV, Nevada; NY, New York; OH, Ohio; MI, Michigan; ID, Idaho;

NH, New Hampshire; WI, Wisconsin; OR, Oregon; MN, Minnesota; WA, Washington]

\begin{tabular}{|c|c|c|c|c|c|c|c|c|c|c|c|c|}
\hline \multirow{2}{*}{$\begin{array}{l}\text { Principal and } \\
\text { regional and (or) } \\
\text { other aquifer } \\
\text { information }\end{array}$} & \multirow{2}{*}{$\begin{array}{l}\text { Network } \\
\text { type }\end{array}$} & \multirow{2}{*}{$\begin{array}{l}\text { Network } \\
\text { name }\end{array}$} & \multirow{2}{*}{$\begin{array}{l}\text { NAWOA well } \\
\text { ID no. }\end{array}$} & \multirow{2}{*}{ State } & \multirow{2}{*}{ County } & \multirow{2}{*}{ Sample date } & \multirow{2}{*}{$\begin{array}{c}\text { Altitude } \\
\text { LS }\end{array}$} & \multirow{2}{*}{$\begin{array}{l}\text { Altitude } \\
\text { datum }\end{array}$} & \multirow{2}{*}{ Water use } & \multirow{2}{*}{$\begin{array}{l}\text { Well } \\
\text { depth } \\
\text { (ft bls) }\end{array}$} & \multicolumn{2}{|c|}{$\begin{array}{c}\text { Depth to perforation } \\
\text { (ft bls) }\end{array}$} \\
\hline & & & & & & & & & & & Top & Bottom \\
\hline $\begin{array}{l}\text { Southeastern } \\
\text { Coastal Plain } \\
\text { aquifer system }\end{array}$ & LUS & santlusrc1 & SANTLUSRC1-15 & $\mathrm{SC}$ & Richland & $8 / 17 / 2016$ & 301 & NGVD 29 & Observation & 53.3 & 48.3 & 53.3 \\
\hline $\begin{array}{l}\text { Southeastern } \\
\text { Coastal Plain } \\
\text { aquifer system }\end{array}$ & LUS & santlusrc1 & SANTLUSRC1-16 & $\mathrm{SC}$ & Richland & $8 / 17 / 2016$ & 240 & NGVD 29 & Observation & 22 & 17 & 22 \\
\hline $\begin{array}{l}\text { Southeastern } \\
\text { Coastal Plain } \\
\text { aquifer system }\end{array}$ & LUS & santlusrc1 & SANTLUSRC1-17 & $\mathrm{SC}$ & Richland & $8 / 18 / 2016$ & 383 & NAVD 88 & Observation & 29.5 & 9 & 24 \\
\hline $\begin{array}{l}\text { Southeastern } \\
\text { Coastal Plain } \\
\text { aquifer system }\end{array}$ & LUS & santlusrc1 & SANTLUSRC1-18 & $\mathrm{SC}$ & Richland & $8 / 22 / 2016$ & 142 & NGVD 29 & Observation & 13.9 & 8.8 & 13.8 \\
\hline $\begin{array}{l}\text { Southeastern } \\
\text { Coastal Plain } \\
\text { aquifer system }\end{array}$ & LUS & santlusrc1 & SANTLUSRC1-19 & $\mathrm{SC}$ & Richland & $8 / 23 / 2016$ & 280 & NGVD 29 & Observation & 23.3 & 18.3 & 23.3 \\
\hline $\begin{array}{l}\text { Southeastern } \\
\text { Coastal Plain } \\
\text { aquifer system }\end{array}$ & LUS & santlusrc1 & SANTLUSRC1-20 & $\mathrm{SC}$ & Richland & $8 / 23 / 2016$ & 170 & NGVD 29 & Observation & 8.6 & 3.6 & 8.6 \\
\hline $\begin{array}{l}\text { Southeastern } \\
\text { Coastal Plain } \\
\text { aquifer system }\end{array}$ & LUS & santlusrc1 & SANTLUSRC1-21 & $\mathrm{SC}$ & Richland & $8 / 24 / 2016$ & 289 & NGVD 29 & Observation & 53.4 & 48.4 & 53.4 \\
\hline $\begin{array}{l}\text { Southeastern } \\
\text { Coastal Plain } \\
\text { aquifer system }\end{array}$ & LUS & santlusrc1 & SANTLUSRC1-22 & $\mathrm{SC}$ & Richland & $8 / 24 / 2016$ & 212 & NGVD 29 & Observation & 16.3 & 11.3 & 16.3 \\
\hline $\begin{array}{l}\text { Southeastern } \\
\text { Coastal Plain } \\
\text { aquifer system }\end{array}$ & LUS & santlusrc1 & SANTLUSRC1-23 & $\mathrm{SC}$ & Lexington & $8 / 25 / 2016$ & 290 & NGVD 29 & Observation & 9 & 4 & 9 \\
\hline $\begin{array}{l}\text { Southeastern } \\
\text { Coastal Plain } \\
\text { aquifer system }\end{array}$ & LUS & santlusrc1 & SANTLUSRC1-24 & $\mathrm{SC}$ & Richland & $8 / 29 / 2016$ & 200 & NGVD 29 & Observation & 8.8 & 3.8 & 8.8 \\
\hline
\end{tabular}


Table 1. Information about wells that have environmental data included in this report.-Continued

[NAWQA, National Water-Quality Assessment; ID, identification; no., number; LS, land surface; ft bls, foot below land surface; lat., latitude, in degrees and minutes; long., longitude in degrees and minutes; PAS, principal aquifer study; FL, Florida; na, not available; NGVD 29, National Geodetic Vertical Datum of 1929; NAVD 88, North American Vertical Datum of 1988; ETN, enhanced trends network; TX, Texas; NM, New Mexico; LUS, land-use study; SC, South Carolina; FPS, flow-path study; MS, Mississippi; VFPS; vertical flow-path study; TN, Tennessee; OK, Oklahoma; AR, Arkansas; CA, California; MO, Missouri; KS, Kansas; MD, Maryland; MSS, modeling support study; DE, Delaware; DC, District of Columbia; MAS, major aquifer study; NV, Nevada; NY, New York; OH, Ohio; MI, Michigan; ID, Idaho; NH, New Hampshire; WI, Wisconsin; OR, Oregon; MN, Minnesota; WA, Washington]

\begin{tabular}{|c|c|c|c|c|c|c|c|c|c|c|c|c|}
\hline \multirow{2}{*}{$\begin{array}{l}\text { Principal and } \\
\text { regional and (or) } \\
\text { other aquifer } \\
\text { information }\end{array}$} & \multirow{2}{*}{$\begin{array}{l}\text { Network } \\
\text { type }\end{array}$} & \multirow{2}{*}{$\begin{array}{l}\text { Network } \\
\text { name }\end{array}$} & \multirow{2}{*}{$\begin{array}{l}\text { NAWQA well } \\
\text { ID no. }\end{array}$} & \multirow{2}{*}{ State } & \multirow{2}{*}{ County } & \multirow{2}{*}{ Sample date } & \multirow{2}{*}{$\begin{array}{l}\text { Altitude } \\
\text { LS }\end{array}$} & \multirow{2}{*}{$\begin{array}{l}\text { Altitude } \\
\text { datum }\end{array}$} & \multirow{2}{*}{ Water use } & \multirow{2}{*}{$\begin{array}{l}\text { Well } \\
\text { depth } \\
\text { (ft bls) }\end{array}$} & \multicolumn{2}{|c|}{$\begin{array}{c}\text { Depth to perforation } \\
\text { (ft bls) }\end{array}$} \\
\hline & & & & & & & & & & & Top & Bottom \\
\hline $\begin{array}{l}\text { Southeastern } \\
\text { Coastal Plain } \\
\text { aquifer system }\end{array}$ & LUS & santlusrc1 & SANTLUSRC1-25 & $\mathrm{SC}$ & Richland & $8 / 30 / 2016$ & 200 & NGVD 29 & Observation & 11.7 & 6.7 & 11.7 \\
\hline $\begin{array}{l}\text { Southeastern } \\
\text { Coastal Plain } \\
\text { aquifer system }\end{array}$ & LUS & santlusrc1 & SANTLUSRC1-26 & $\mathrm{SC}$ & Richland & $8 / 31 / 2016$ & 277 & NAVD 88 & Observation & 21.5 & 11 & 21 \\
\hline $\begin{array}{l}\text { Southeastern } \\
\text { Coastal Plain } \\
\text { aquifer system }\end{array}$ & LUS & santlusrc1 & SANTLUSRC1-27 & $\mathrm{SC}$ & Richland & $9 / 1 / 2016$ & 215 & NGVD 29 & Observation & 50 & 45 & 50 \\
\hline
\end{tabular}

aThere was no information available about the bottom of perforation but was assumed to be the bottom of well (Well_depth).

bThe depth to the bottom was less than the depth to the top of the open interval and was assumed to be the well depth. 


\section{Appendix 1. Information Contained in Previous Reports in This Series}

A list of reports in this series and the networks discussed are listed in table 1.1.

Table 1.1. Index to which report contains each network description.

\begin{tabular}{|c|c|c|c|}
\hline Reference & $\begin{array}{l}\text { Year network } \\
\text { was sampled }\end{array}$ & $\begin{array}{l}\text { Network name } \\
\text { abbreviation }\end{array}$ & Network name used in report \\
\hline \multirow[t]{16}{*}{$\begin{array}{l}\text { Arnold and others } \\
\qquad(2016 \mathrm{a}, \mathrm{b})\end{array}$} & 2013 & acfbluscr3 & $\begin{array}{l}\text { Apalachicola-Chattahoochee-Flint River Basin agricultural land-use study } \\
\text { network }\end{array}$ \\
\hline & 2013 & albesus 7 & Albemarle-Pamlico Drainage Basin major aquifer study network \\
\hline & 2013 & bnrfpas 1 & Basin and Range Basin-Fill Aquifers principal aquifer study network \\
\hline & 2013 & clowpas 1 & Coastal Lowlands Aquifer System principal aquifer study network \\
\hline & 2012 & dlmvluscr1 & Delmarva Peninsula agricultural land-use study network \\
\hline & 2013 & dlmvsus 1 & Delmarva Peninsula major aquifer study network \\
\hline & 2013 & edtretn 1 & Edwards-Trinity Aquifer System enhanced trends network \\
\hline & 2012 & gafllusrc1a & Georgia-Florida urban land-use study network near Tampa, Florida \\
\hline & 2013 & metxetn 1 & Mississippi Embayment Aquifer System enhanced trends network \\
\hline & 2012 & nacppas 1 & Northern Atlantic Coastal Plain Aquifer System principal aquifer study networks \\
\hline & 2013 & nacppas 2 & Northern Atlantic Coastal Plain Aquifer System principal aquifer study networks \\
\hline & 2013 & nvbrlusrc1 & $\begin{array}{l}\text { Nevada Basin and Range urban land-use study network near Reno and Carson } \\
\text { City, Nevada }\end{array}$ \\
\hline & 2013 & sanjlusorla & San Joaquin Valley agricultural land-use study network \\
\hline & 2013 & secppas 1 & Southeastern Coastal Plain Aquifer System principal aquifer study network \\
\hline & 2013 & spltluscr1 & South Platte River agricultural land-use study network \\
\hline & 2013 & vpdcpas 1 & $\begin{array}{l}\text { Valley and Ridge, and Piedmont and Blue Ridge Carbonate-Rock Aquifers prin- } \\
\text { cipal aquifer study network }\end{array}$ \\
\hline
\end{tabular}


Table 1.1. Index to which report contains each network description.-Continued

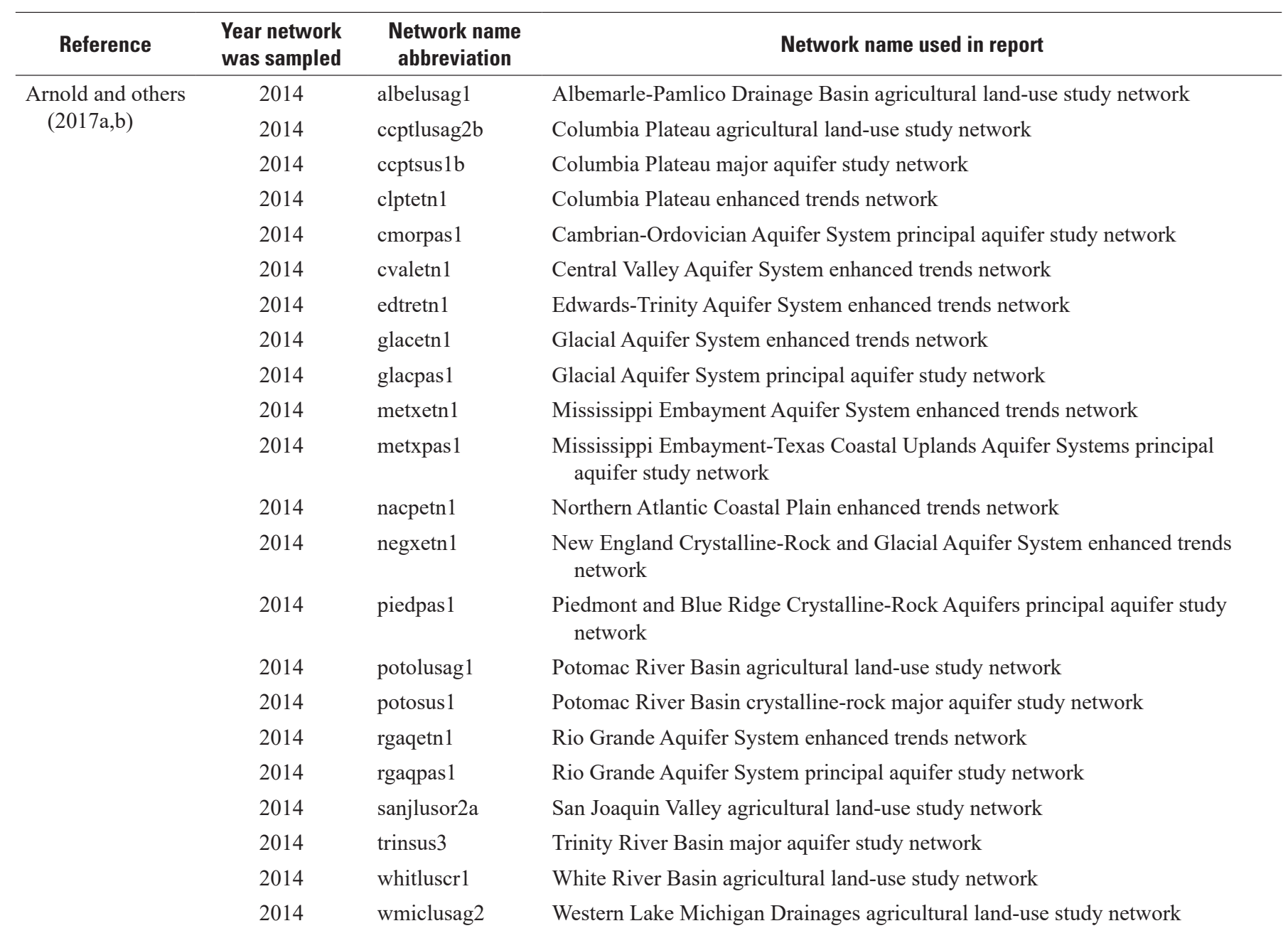


Table 1.1. Index to which report contains each network description.-Continued

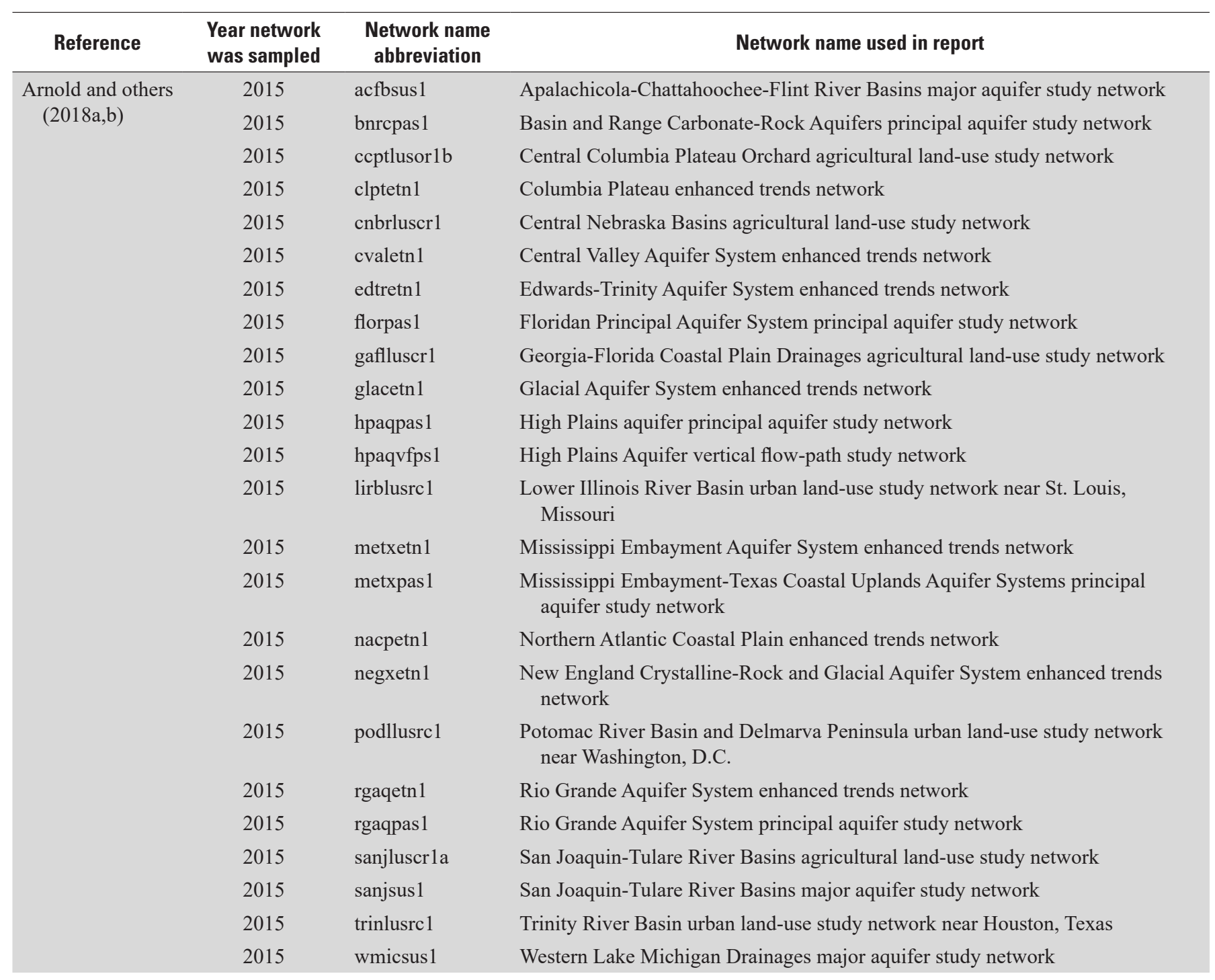


Table 1.1. Index to which report contains each network description.-Continued

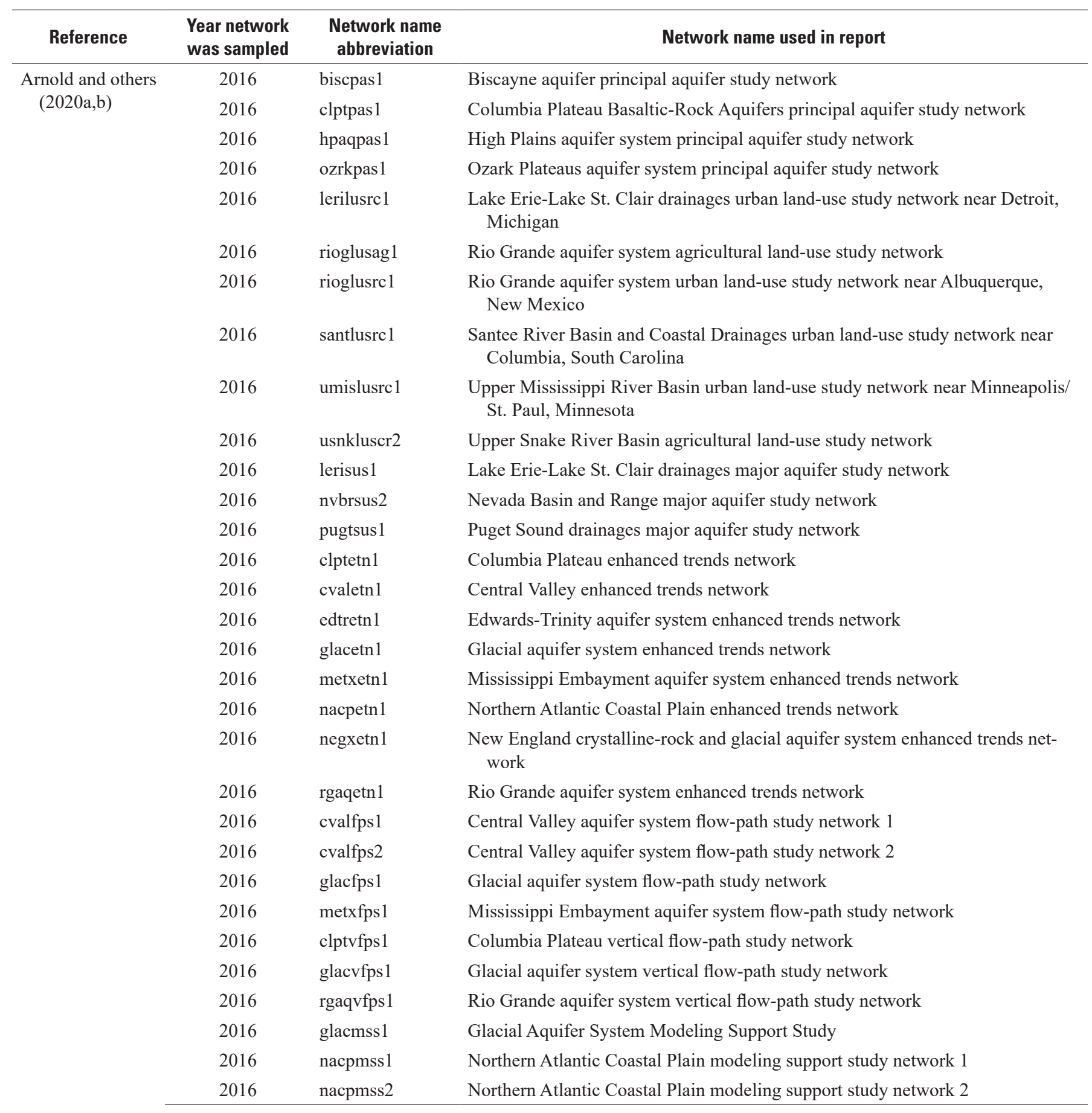




\section{References Cited}

Arnold, T.L., Bexfield, L.M., Musgrove, M., Erickson, M.L., Kingsbury, J.A., Tesoriero, A.J., Kulongoski, J.T., and Belitz, K., 2020a, Groundwater-quality and select qualitycontrol data from the National Water-Quality Assessment Project, January through December 2016, and previously unpublished data from 2012 to 2015: U.S. Geological Survey Data Series 1124, 135 p., https://doi.org/10.3133/ ds 1124.

Arnold, T.L., Bexfield, L.M., Musgrove, M., Lindsey, B.D., Stackelberg, P.E., Barlow, J.R., DeSimone, L.A., Kulongoski, J.T., Kingsbury, J.A., Ayotte, J.D., Fleming, B.J., and Belitz, K., 2017a, Datasets from groundwater quality data from the National Water Quality Assessment Project, January through December 2014 and select qualitycontrol data from May 2012 through December 2014: U.S. Geological Survey data release, accessed March 28, 2018, at https://doi.org/10.5066/F7W0942N.

Arnold, T.L., Bexfield, L.M., Musgrove, M., Lindsey, B.D., Stackelberg, P.E., Barlow, J.R., DeSimone, L.A., Kulongoski, J.T., Kingsbury, J.A., Ayotte, J.D., Fleming, B.J., and Belitz, K., 2017b, Groundwater-quality data from the National Water-Quality Assessment Project, January through December 2014 and select quality-control data from May 2012 through December 2014: U.S. Geological Survey Data Series 1063, 83 p., accessed March 28, 2018, at https://doi.org/10.3133/ds1063.

Arnold, T.L., Bexfield, L.M., Musgrove, M., Stackelberg, P.E., Lindsey, B.D., Kingsbury, J.A., Kulongoski, J.T., and Belitz, K., 2018a, Groundwater-quality and select qualitycontrol data from the National Water-Quality Assessment Project, January through December 2015, and previously unpublished data from 2013 to 2014: U.S. Geological Survey Data Series 1087, 68 p., accessed February 2020 at https://doi.org/10.3133/ds1087.
Arnold, T.L., Bexfield, L.M., Musgrove, M., Stackelberg, P.E., Lindsey, B.D., Kingsbury, J.A., Kulongoski, J.T., Belitz, K., and Sharpe, J.B., 2018b, Datasets from groundwaterquality and select quality-control data from the National Water-Quality Assessment Project, January through December 2015 and previously unpublished data from 2013-2014: U.S. Geological Survey data release, accessed September 4, 2019, at https://doi.org/10.5066/F7XK8DHK.

Arnold, T.L., Bexfield, L.M., Musgrove, M., Stackelberg, P.E., Lindsey, B.D., Kingsbury, J.A., Kulongoski, J.T., Belitz, K., and Sharpe, J.B., 2020b, Datasets from groundwater-quality and select quality-control data from the National Water-Quality Assessment Project, January through December 2016, and previously unpublished data from 2013 to 2015: U.S. Geological Survey data release, https://doi.org/10.5066/P9W4RR74.

Arnold, T.L., DeSimone, L.A., Bexfield, L.M., Lindsey, B.D., Barlow, J.R., Kulongoski, J.T., Musgrove, M., Kingsbury, J.A., and Belitz, K., 2016a, Groundwater quality data from the National Water-Quality Assessment Project, May 2012 through December 2013: U.S. Geological Survey Data Series 997, 56 p., accessed March 28, 2018, at https://doi.org/10.3133/ds997.

Arnold, T.L., DeSimone, L.A., Bexfield, L.M., Lindsey, B.D., Barlow, J.R., Kulongoski, J.T., Musgrove, M., Kingsbury, J.A., and Belitz, K., 2016b, Groundwater quality data from the National Water Quality Assessment Project, May 2012 through December 2014 and select quality-control data from May 2012 through December 2013: U.S. Geological Survey data release, accessed March 28, 2018, at https://doi.org/ 10.5066/F7HQ3X18. 
Table 2.1. Well depth by study network.

[ETN, enhanced trends network; nc, not calculated; LUS, land-use study; MAS, major aquifer study; PAS, principal aquifer study; VFPS, vertical flow-path study]

\begin{tabular}{|c|c|c|c|c|c|c|c|c|c|c|}
\hline \multirow{2}{*}{$\begin{array}{l}\text { Network } \\
\text { type }\end{array}$} & \multirow{2}{*}{$\begin{array}{l}\text { Network } \\
\text { name }\end{array}$} & \multirow{2}{*}{$\begin{array}{c}\text { Number of wells in } \\
\text { network with data } \\
\text { presented in this report }\end{array}$} & \multirow{2}{*}{$\begin{array}{c}\text { Number of wells with } \\
\text { well depth data }\end{array}$} & \multicolumn{7}{|c|}{ Well depth, in feet below land surface } \\
\hline & & & & Minimum & $\begin{array}{c}\text { 10th } \\
\text { percentile }\end{array}$ & $\begin{array}{c}\text { 25th } \\
\text { percentile }\end{array}$ & Median & $\begin{array}{c}\text { 75th } \\
\text { percentile }\end{array}$ & $\begin{array}{c}\text { 95th } \\
\text { percentile }\end{array}$ & Maximum \\
\hline ETN & clptetn1 & 3 & 3 & 80 & nc & nc & 170 & $\mathrm{nc}$ & nc & 1,116 \\
\hline ETN & cvaletn1 & 3 & 3 & 234 & nc & $\mathrm{nc}$ & 320 & $\mathrm{nc}$ & $\mathrm{nc}$ & 620 \\
\hline ETN & edtretn 1 & 3 & 3 & 300 & nc & $\mathrm{nc}$ & 550 & $\mathrm{nc}$ & $\mathrm{nc}$ & 1,500 \\
\hline ETN & glacetn 1 & 5 & 5 & 34.5 & nc & $\mathrm{nc}$ & 83 & nc & $\mathrm{nc}$ & 125 \\
\hline ETN & metxetn1 & 2 & 2 & 90 & $\mathrm{nc}$ & $\mathrm{nc}$ & 357 & nc & $\mathrm{nc}$ & 624 \\
\hline ETN & nacpetn1 & 3 & 3 & 22 & nc & nc & 119 & nc & $\mathrm{nc}$ & 135 \\
\hline ETN & negxetn1 & 3 & 3 & 83 & $\mathrm{nc}$ & $\mathrm{nc}$ & 176.3 & $\mathrm{nc}$ & $\mathrm{nc}$ & 492 \\
\hline ETN & rgaqetn1 & 3 & 3 & 22 & $\mathrm{nc}$ & nc & 22.6 & $\mathrm{nc}$ & nc & 60 \\
\hline FPS & cvalfps1 & 20 & 20 & 70 & 79.7 & 87 & 149 & 187.25 & 268 & 268 \\
\hline FPS & cvalfps2 & 21 & 21 & 100 & 132 & 180 & 300 & 455 & 530 & 532 \\
\hline FPS & glacfps1 & $26^{\mathrm{a}}$ & 27 & 1.25 & 1.3 & 1.3 & 33 & 62 & 90 & 99 \\
\hline FPS & metxfps1 & 22 & 22 & 23 & 77.19 & 92 & 177 & 267.5 & 597.2 & 624 \\
\hline LUS & lerilusrc1 & 28 & 28 & 10.5 & 16.03 & 19.6 & 26.9 & 32.55 & 46.445 & 68.5 \\
\hline LUS & rioglusag1 & 24 & 24 & 14.9 & 16.34 & 18.275 & 22.75 & 32.3 & 36.625 & 37.2 \\
\hline LUS & rioglusrc1 & 27 & 27 & 12.5 & 19.38 & 20.8 & 26.5 & 30.5 & 48.06 & 57 \\
\hline LUS & santlusrc1 & 27 & 27 & 8.6 & 10.26 & 13.7 & 21.5 & 40.15 & 53.37 & 54 \\
\hline LUS & umislusrc1 & 30 & 30 & 9 & 11.98 & 15 & 18 & 23.75 & 28.775 & 33.5 \\
\hline LUS & usnkluscr2 & 31 & 30 & 125 & 163.5 & 197.75 & 256 & 283.75 & 360.5 & 469 \\
\hline MAS & lerisus1 & 24 & 24 & 35 & 61.6 & 72.75 & 90.5 & 107.25 & 168.2 & 205 \\
\hline MAS & nvbrsus2 & 30 & 30 & 105 & 162.8 & 236.5 & 347 & 569.75 & 789.35 & 815 \\
\hline MAS & pugtsus 1 & 29 & 29 & 22.5 & 40 & 60 & 78 & 112 & 290.8 & 309 \\
\hline MSS & glacmss1 & 30 & 27 & 45 & 52.6 & 62.5 & 90 & 140.5 & 190.9 & 245 \\
\hline MSS & nacpmss 1 & 20 & 20 & 60 & 152 & 194.25 & 292.5 & 369.25 & 608.9 & 645 \\
\hline MSS & nacpmss2 & 25 & 25 & 200 & 257.6 & 303 & 466 & 626 & 731.4 & 900 \\
\hline PAS & biscpas1 & 40 & 31 & 22 & 40 & 60 & 91 & 113.5 & 140 & 152 \\
\hline PAS & clptpas1 & 60 & 57 & 91 & 222 & 309 & 484 & 743 & 1,328 & 2,434 \\
\hline PAS & hpaqpas1 & 17 & 17 & 180 & 196.6 & 242 & 321 & 395 & 731.2 & 812 \\
\hline PAS & ozrkpas1 & 60 & 57 & 370 & 568.6 & 900 & 1,115 & 1,425 & $1,943.4$ & 3,420 \\
\hline VFPS & clptvfps1 & 19 & 19 & 19 & 27.5 & 35 & 88 & 175 & 772.3 & 1,000 \\
\hline VFPS & glacvfps1 & 43 & 43 & 10.5 & 16.62 & 22.55 & 33.9 & 76.25 & 136.9 & 172 \\
\hline VFPS & rgaqvfps 1 & 19 & 19 & 18.3 & 20.1 & 27.15 & 34.8 & 53.4 & 160.22 & 254 \\
\hline
\end{tabular}


Table 2.2. Length of open interval by study network.

[ETN, enhanced trends network; nc, not calculated; LUS, land-use study; MAS, major aquifer study; PAS, principal aquifer study; na, not available; VFPS, vertical flow-path study]

\begin{tabular}{|c|c|c|c|c|c|c|c|c|c|c|}
\hline \multirow{2}{*}{$\begin{array}{c}\text { Network } \\
\text { type }\end{array}$} & \multirow{2}{*}{$\begin{array}{c}\text { Network } \\
\text { name }\end{array}$} & \multirow{2}{*}{$\begin{array}{c}\text { Number of wells in } \\
\text { network with data } \\
\text { presented in this report }\end{array}$} & \multirow{2}{*}{$\begin{array}{l}\text { Number of wells } \\
\text { with open interval } \\
\text { data }\end{array}$} & \multicolumn{7}{|c|}{ Length of open interval, in feet } \\
\hline & & & & Minimum & $\begin{array}{c}\text { 10th } \\
\text { percentile }\end{array}$ & $\begin{array}{c}\text { 25th } \\
\text { percentile }\end{array}$ & Median & $\begin{array}{c}\text { 75th } \\
\text { percentile }\end{array}$ & $\begin{array}{c}\text { 95th } \\
\text { percentile }\end{array}$ & Maximum \\
\hline ETN & clptetn 1 & 3 & 3 & 1 & $\mathrm{nc}$ & $\mathrm{nc}$ & 26 & $\mathrm{nc}$ & $\mathrm{nc}$ & 174 \\
\hline ETN & cvaletn 1 & 3 & 3 & 10 & $\mathrm{nc}$ & $\mathrm{nc}$ & 150 & $\mathrm{nc}$ & $\mathrm{nc}$ & 200 \\
\hline ETN & edtretn1 & 3 & 3 & 80 & $\mathrm{nc}$ & $\mathrm{nc}$ & 230 & $\mathrm{nc}$ & $\mathrm{nc}$ & 233 \\
\hline ETN & glacetn 1 & 5 & 4 & 3 & $\mathrm{nc}$ & $\mathrm{nc}$ & 5 & $\mathrm{nc}$ & nc & 10 \\
\hline ETN & metxetn1 & 2 & 2 & 10 & $\mathrm{nc}$ & $\mathrm{nc}$ & 57 & $\mathrm{nc}$ & nc & 104 \\
\hline ETN & nacpetn1 & 3 & 3 & 3 & $\mathrm{nc}$ & $\mathrm{nc}$ & 19 & $\mathrm{nc}$ & $\mathrm{nc}$ & 50 \\
\hline ETN & negxetn1 & 3 & 2 & 10 & $\mathrm{nc}$ & $\mathrm{nc}$ & 207 & $\mathrm{nc}$ & nc & 404 \\
\hline ETN & rgaqetn1 & 3 & 3 & 10 & $\mathrm{nc}$ & $\mathrm{nc}$ & 10 & $\mathrm{nc}$ & $\mathrm{nc}$ & 20 \\
\hline FPS & cvalfps 1 & 20 & 20 & 5 & 5 & 5 & 5 & 5 & 5 & 5 \\
\hline FPS & cvalfps 2 & 21 & 20 & 0 & 0 & 17.5 & 60 & 240 & 296.3 & 340 \\
\hline FPS & glacfps1 & 26 & 26 & 0.05 & 0.1 & 0.1 & 3 & 3 & 8.75 & 10 \\
\hline FPS & metxfps 1 & 22 & 21 & 10 & 10 & 10 & 20 & 44 & 100 & 104 \\
\hline LUS & lerilusrc1 & 28 & 28 & 4.5 & 4.5 & 4.5 & 4.5 & 4.5 & 6.625 & 8.1 \\
\hline LUS & rioglusag1 & 24 & 24 & 9.3 & 9.3 & 9.3 & 9.4 & 9.6 & 10 & 10 \\
\hline LUS & rioglusrc1 & 27 & 27 & 5 & 7.76 & 10 & 10 & 10 & 15 & 15 \\
\hline LUS & santlusrc1 & 27 & 27 & 5 & 5 & 5 & 5 & 5 & 13.5 & 15 \\
\hline LUS & umislusrc1 & 30 & 30 & 4.5 & 5 & 5 & 5 & 5 & 5 & 10 \\
\hline LUS & usnkluscr2 & 31 & 25 & 2 & 35.8 & 58 & 171 & 256 & 322.8 & 336.5 \\
\hline MAS & lerisus1 & 24 & 24 & 3 & 3 & 4 & 4 & 4 & 8 & 10 \\
\hline MAS & nvbrsus2 & 30 & 30 & 10 & 10 & 68.75 & 193 & 271.25 & 509.2 & 670 \\
\hline MAS & pugtsus1 & 29 & 27 & 0 & 0 & 0.5 & 5 & 10 & 48.4 & 60 \\
\hline MSS & glacmss1 & 30 & 14 & 4 & 8.63 & 20 & 25 & 31.5 & 41.75 & 45 \\
\hline MSS & nacpmss1 & 20 & 20 & 5 & 6.9 & 10 & 20 & 32.5 & 112 & 150 \\
\hline MSS & nacpmss2 & 25 & 24 & 30 & 47.9 & 50.75 & 59.5 & 60 & 60.68 & 103.4 \\
\hline PAS & biscpas1 & 40 & 25 & 3 & 5.4 & 7 & 22 & 40 & 64.6 & 71 \\
\hline PAS & clptpas1 & 60 & 51 & 0 & 70 & 109 & 207 & 342 & 695.75 & $1,296.70$ \\
\hline PAS & hpaqpas 1 & 17 & 7 & 82 & 86.8 & 125 & 161 & 187.5 & 242.5 & 265 \\
\hline PAS & ozrkpas1 & 60 & 51 & 8 & 330 & 479 & 621 & 936 & 1,200 & $1,968.50$ \\
\hline VFPS & clptvfps1 & 19 & 18 & 3 & 5 & 5 & 5 & 140 & 463.4 & 681 \\
\hline VFPS & glacvfps1 & 43 & 43 & 4 & 4 & 4.25 & 4.5 & 4.5 & 7.35 & 10 \\
\hline VFPS & rgaqvfps 1 & 19 & 19 & 5 & 5 & 7.5 & 10 & 12.5 & 21.7 & 82 \\
\hline
\end{tabular}




\section{Appendix 3. High-Frequency Data from Enhanced Trends Networks}

High-frequency data collected at enhanced trends network sites are available from the National Water Information System (U.S. Geological Survey, 2018) online database (table 3.1). The links in table 3.1 below provide access to the high-frequency data on the web. To access the data for the period covered by this report, the user should open the National Water Information System web page at https://doi.org/10.5066/F7P55KJN. The user should then change the begin and end dates to retrieve the data for the period January 1, 2015, to December 31, 2015.
Some of the enhanced trends network sites may have different equipment installed and may collect different parameters than others. Additionally, some sites have missing records for various properties because of equipment failures at various times during the data-collection period. Locations of the enhanced trends networks are shown in figures 15-16 of this report.

Table 3.1. Web links to data collected at a high frequency from enhanced trends networks.

[See figures 15-16 of this report for locations of enhanced trends networks. NAWQA, National Water-Quality Assessment]

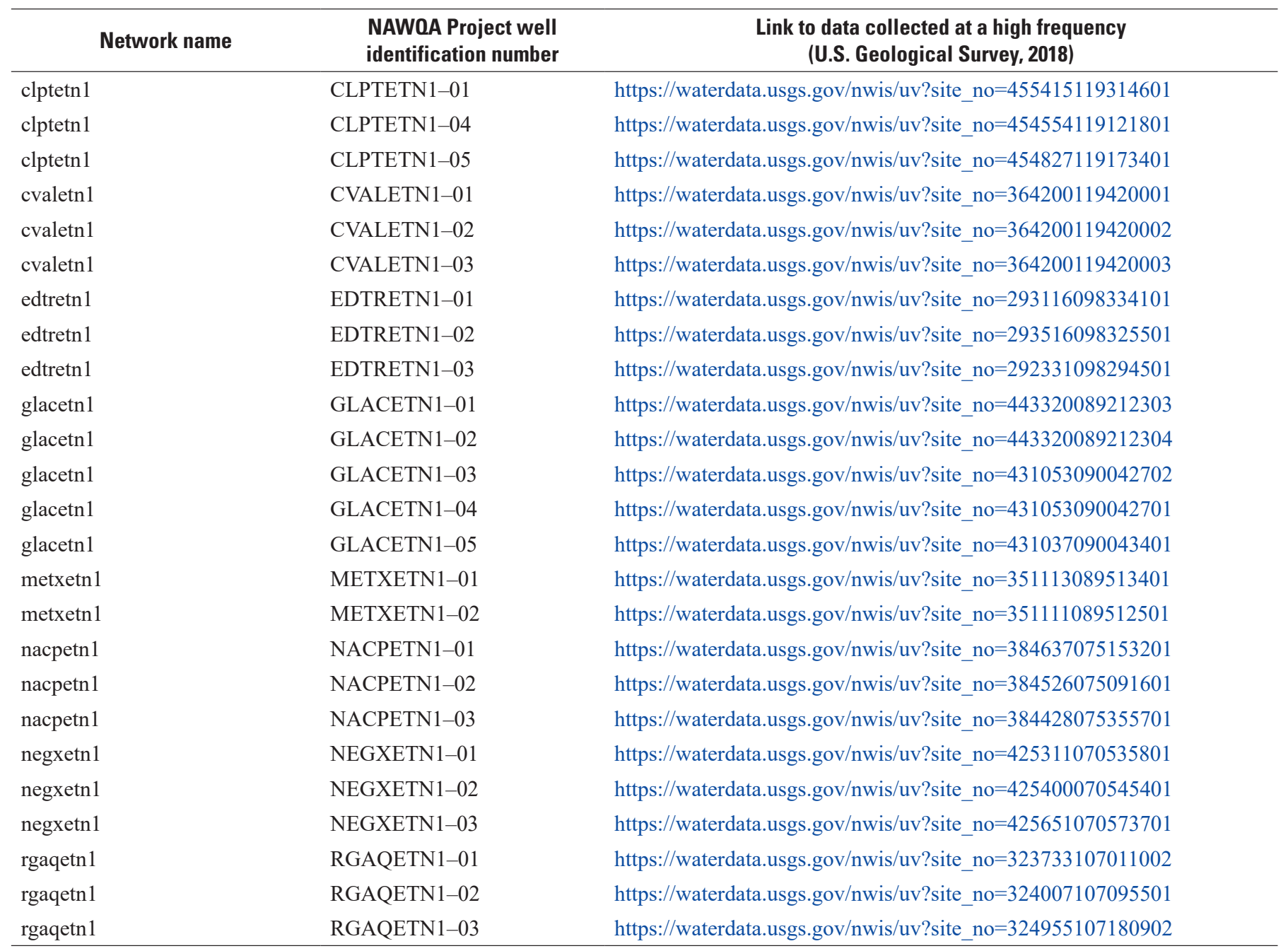




\section{Reference Cited}

U.S. Geological Survey, 2018, USGS water data for the Nation: U.S. Geological Survey National Water Information System database, accessed December 18, 2018, at https://doi.org/10.5066/F7P55KJN. 


\section{Appendix 4. Quality-Control Samples and Data Analysis}

\section{Samples}

Quality-control (QC) samples are routinely collected along with the environmental groundwater samples. The third cycle of the National Water-Quality Assessment (NAWQA) groundwater studies began in 2013, but a small pilot study was completed in 2012. The entire third cycle sampling period currently is May 2012-December 2016; this period is hereafter referred to as "the cycle 3 sampling period." Data from the environmental and QC blank and replicate samples from the 2012-13 sampling period were presented in Arnold and others $(2016 \mathrm{a}, \mathrm{b})$ and from the 2014 sampling period were presented in Arnold and others (2017a,b). Data from the environmental and QC blank samples from the 2015 sampling period were presented in Arnold and others (2018a,b). The Arnold and others $(2017 \mathrm{a}, \mathrm{b})$ publications also presented data for selected spike samples collected in 2012-14. This current report presents a summary of QC samples from the entire cycle 3 sampling period (May 2012-December 2016) as well as the January-December 2016 sampling period covered by this report. A summary of results from blank samples collected during the sampling period January-December 2016 is provided in table 4.1, and a summary for the cycle 3 sampling period is provided in table 4.2. A summary of replicate samples, an analysis of the variability in detections and concentrations of selected analytes from replicate samples, and a summary of spike samples for the entire sampling period May 2012-December 2016 are provided in tables 4.3 through 4.9. Data from the environmental and QC samples from the January-December 2016 sampling period are presented in tables 4.10-4.19 of Arnold and others (2020).

Table 4.1. Summary of results for field blanks collected by the National Water-Quality Assessment Project from January to December 2016.

[DOC, dissolved organic carbon; VOC, volatile organic compound; HHB, human-health benchmark; --, not applicable; SMCL, secondary maximum contaminant level]

\begin{tabular}{|c|c|c|c|c|c|}
\hline Type of summary & $\begin{array}{l}\text { Major and } \\
\text { minor ele- } \\
\text { ments }\end{array}$ & $\begin{array}{l}\text { Trace ele- } \\
\text { ments }\end{array}$ & Nutrients and DOC & VOCs & $\begin{array}{l}\text { Pesticide com- } \\
\text { pounds }\end{array}$ \\
\hline Total number of blank samples & 48 & 80 & 43 to 82 & 87 & 29 \\
\hline Number of field blanks & 43 & 46 & 41 to 47 & 38 & 26 \\
\hline Number of constituents analyzed & 10 & 22 & 6 & 85 & 225 \\
\hline Number of constituents detected in field blanks & 9 & 20 & 4 & 19 & 1 \\
\hline $\begin{array}{l}\text { Number of constituents detected in field blanks } \\
\text { that have an SMCL }\end{array}$ & 4 & 5 & 0 & 0 & 0 \\
\hline $\begin{array}{l}\text { Largest ratio of the maximum concentration in } \\
\text { a field blank to the corresponding HHB, in } \\
\text { percent }\end{array}$ & 2 & 4.5 & 0.16 & 0.36 & -- \\
\hline
\end{tabular}


Table 4.2. Summary of results for field blanks collected by the National Water-Quality Assessment Project from May 2012 to December 2016.ww

[DOC, dissolved organic carbon; VOC, volatile organic compound; HHB, human-health benchmark; SMCL, secondary maximum contaminant level; --, not applicable]

\begin{tabular}{|c|c|c|c|c|c|c|}
\hline Type of summary & $\begin{array}{l}\text { Major and } \\
\text { minor } \\
\text { elements }\end{array}$ & $\begin{array}{c}\text { Trace } \\
\text { elements }\end{array}$ & $\begin{array}{l}\text { Nutrients } \\
\text { and DOC }\end{array}$ & VOCs & $\begin{array}{l}\text { Pesticide } \\
\text { compounds }\end{array}$ & $\begin{array}{l}\text { Special- } \\
\text { interest } \\
\text { analytes }\end{array}$ \\
\hline Total number of blank samples & 227 & 458 & 209 to 457 & 564 & 184 & 6 to 54 \\
\hline Number of field blanks & 208 & 219 & 200 to 220 & 200 & 165 & 6 to 20 \\
\hline Number of constituents analyzed & 10 & 22 & 6 & 129 & 272 & 7 \\
\hline Number of constituents detected in field blanks & 10 & 21 & 6 & 27 & 27 & 3 \\
\hline $\begin{array}{l}\text { Number of constituents detected in field blanks } \\
\text { that have an SMCL }\end{array}$ & 4 & 5 & 0 & 0 & 0 & 0 \\
\hline $\begin{array}{l}\text { Largest ratio of the maximum concentration in } \\
\text { a field blank to the corresponding HHB, in } \\
\text { percent }\end{array}$ & 2 & 4.8 & 0.67 & 2.4 & 0.25 & 750 \\
\hline
\end{tabular}

\section{Blank Sample Approach}

Blank samples are QC samples that are used to determine if water samples become contaminated during sample collection, field processing, transport, or laboratory analysis. Blank samples are collected using blank water that has been prepared to be free of detectable concentrations of the constituents of interest. An equipment blank generally is collected in a controlled environment (such as a laboratory) before field sampling begins and is intended to evaluate the suitability of the equipment and equipment cleaning protocols for the established data-quality requirements. A field blank is subjected to all the same aspects of sample collection, field processing, preservation, transportation, and laboratory handling as an environmental sample and is intended to evaluate the potential for these procedures to be sources of contamination. A source solution blank is a sample of the water used to collect the equipment and field blanks and is intended to verify that the blank water itself has no detectable concentrations of the constituents of interest. Because field blanks are collected under conditions most comparable to conditions affecting environmental samples, these blanks are most directly representative of potential sources of contamination to environmental samples and were the focus of this initial evaluation of blank-sample results.

Results of the initial evaluation of data from field blanks for major and trace elements, nutrients, volatile organic compounds (VOCs), and pesticide compounds collected during the 2016 sampling period of January-December 2016 and the cycle 3 sampling period are presented in this report. Data from 2016 are published in this report, data from 2012 to 2013 were published in Arnold and others (2016a,b), and data from 2014 were published in Arnold and others (2017a,b). About 70 to 75 percent of the field blanks collected for each of these constituents during the cycle 3 sampling period have been associated with groundwater sites that are sampled using a dedicated pump (primarily public-supply and domestic wells), and the rest have been associated with groundwater sites that are sampled using a portable sampling pump (monitoring wells). The objective of this initial evaluation of field blanks was to determine if environmental concentrations of these constituents as reported by the relevant laboratories are suitable for comparison to their corresponding human-health benchmarks (HHBs) or to U.S. Environmental Protection Agency (EPA) secondary maximum contaminant levels (SMCLs) if HHBs have not been established. The HHBs are a set of health-based comparison thresholds that include EPA maximum contaminant levels (MCLs), health-based screening levels (HBSLs), and humanhealth benchmarks for pesticides (HHBPs). Further evaluation of results for blank samples, such as through methods used by Olsen and others (2010), Bender and others (2011), Fram and others (2012), or Davis and others (2014), would be needed to determine if inadvertent contamination of samples with certain constituents would affect the interpretation of environmental concentrations of those constituents for objectives other than those presented in this report. 
Table 4.3. Summary of results for replicate samples collected by the National Water-Quality Assessment Project from May 2012 to December 2016.

[VOC, volatile organic compound; mg/L, milligram per liter; $\mu \mathrm{g} / \mathrm{L}$, microgram per liter; ng/L, nanogram per liter; $\mathrm{pCi} / \mathrm{L}$, picocurie per liter]

\begin{tabular}{|c|c|c|c|c|c|c|c|}
\hline Type of summary & $\begin{array}{l}\text { Major and minor } \\
\text { elements and } \\
\text { physical parameters }\end{array}$ & Trace elements & $\begin{array}{l}\text { Nutrients and } \\
\text { dissolved organic } \\
\text { carbon }\end{array}$ & VOCs & $\begin{array}{l}\text { Pesticide } \\
\text { compounds }\end{array}$ & $\begin{array}{l}\text { Radio-logical } \\
\text { analytes }\end{array}$ & $\begin{array}{l}\text { Special- } \\
\text { interest } \\
\text { analytes }\end{array}$ \\
\hline Total number of replicate samples & 58 to 119 & 128 & 103 to 120 & 110 & 142 & 46 to 86 & 4 to 10 \\
\hline $\begin{array}{l}\text { Number of samples included in analysis of replicate } \\
\text { results (2013-16 samples for VOCs and pesticide } \\
\text { compounds) }\end{array}$ & 58 to 119 & 128 & 103 to 120 & 105 & 138 & 46 to 86 & 10 \\
\hline $\begin{array}{l}\text { Total number of analytes included in analysis of } \\
\text { replicate results }\end{array}$ & 14 & 22 & 6 & 85 & 225 & 8 & 1 \\
\hline $\begin{array}{l}\text { Number of analytes that include censored values } \\
\text { and have at least } 10 \text { replicate pairs without con- } \\
\text { sistent non-detections }\end{array}$ & 5 & 19 & 6 & 4 & 11 & 7 & 0 \\
\hline $\begin{array}{l}\text { Range in mean detection rate for replicate pairs } \\
\text { having at least } 1 \text { detection, in percent }\end{array}$ & $90.6-100$ & $80.0-100$ & $84.6-99.5$ & $93.8-100$ & $78.1-97.8$ & $81.8-94.3$ & -- \\
\hline $\begin{array}{l}\text { Number of analytes with mean detection rate less } \\
\text { than } 75 \text { percent }\end{array}$ & 0 & 0 & 0 & 0 & 0 & 0 & -- \\
\hline $\begin{array}{l}\text { Range in percentage of replicate pairs with incon- } \\
\text { sistent detections }\end{array}$ & $0-18.8$ & $0-40.0$ & $0.9-30.8$ & $0-12.5$ & $4.3-43.8$ & $11.4-36.5$ & -- \\
\hline $\begin{array}{l}\text { Number of analytes with percentage of replicate } \\
\text { pairs with inconsistent detections greater than } \\
50 \text { percent }\end{array}$ & 0 & 0 & 0 & 0 & 0 & 0 & -- \\
\hline $\begin{array}{l}\text { Number of analytes that have at least } 10 \text { replicate } \\
\text { pairs with consistent analyte detections }\end{array}$ & 14 & 18 & 6 & 4 & 9 & 8 & 0 \\
\hline $\begin{array}{l}\text { Range in standard deviation for lower concentration } \\
\text { range of two-range model }\end{array}$ & $0.0014-7.3 \mathrm{mg} / \mathrm{L}$ & $0.0022-1.3 \mu \mathrm{g} / \mathrm{L}$ & $0.0004-0.039 \mathrm{mg} / \mathrm{L}$ & $0.0013-0.013 \mu \mathrm{g} / \mathrm{L}$ & $1.1-19 \mathrm{ng} / \mathrm{L}$ & $0.13-12 \mathrm{pCi} / \mathrm{L}$ & -- \\
\hline $\begin{array}{l}\text { Range in relative standard deviation for higher con- } \\
\text { centration range of two-range model, in percent }\end{array}$ & $0.24-5.7$ & $1.2-9.3$ & $0.98-10.8$ & 5.8 & $3.6-13$ & $2.9-14$ & -- \\
\hline
\end{tabular}




\section{Blank Sample Counts}

The total number of blank samples and the number of field blanks collected for groundwater sites differs by constituent group during the 2016 sampling period and the cycle 3 sampling period (tables 4.1 and 4.2, respectively). Data for all blank samples from the 2016 sampling period are presented in tables 4.10-4.17 of Arnold and others (2020). All blank samples collected during the cycle 3 sampling period were analyzed using the corresponding laboratory methods listed in table 2 of Arnold and others (2020, 2016a, 2017a, 2018a). Of the 564 VOC blank samples collected during the cycle 3 sampling period (table 4.2), 26 were collected in 2012 or early 2013 and analyzed for an older analytical schedule using purge and trap gas chromatography/mass spectrometry (Gilliom and others, 2006; Zogorski and others, 2006); and 538 were collected in 2013-16 and analyzed using the most recent analytical schedule and laboratory methods (purge and trap gas chromatography/mass spectrometry and heated purge and trap gas chromatography/mass spectrometry). Of the 184 pesticide blank samples collected during the cycle 3 sampling period (table 4.2), 8 were collected in 2012 and analyzed for an older analytical schedule using gas chromatography/mass spectrometry; and 176 were collected in 2013-16 and analyzed using the most recent analytical schedule and laboratory method (direct aqueous injection liquid chromatography tandem mass spectrometry). One blank sample collected in 2014 was analyzed using both an older and the most recent analytical schedule. Not included in table 4.1 are sample counts for special analytes collected only in selected well networks during the cycle 3 sampling period: arsenic species, perchlorate, and hexavalent chromium. In January-December 2016, no field blanks were collected for arsenic species, and only two each were collected for perchlorate and hexavalent chromium.

\section{Constituent Concentrations in Blank Samples}

Of the 10 major or minor elements included in laboratory analysis (not including analysis for dissolved-solids concentration), 9 were detected in at least 1 field blank collected in 2016 (table 4.1); all 10 elements were detected in at least 1 field blank collected during the cycle 3 sampling period (table 4.2). Only one of the major or minor elements detected during the cycle 3 sampling period (fluoride) had an HHB (table 2 of Arnold and others, 2020); four (chloride, fluoride, sulfate, and iron) had SMCLs. The maximum concentration for fluoride in any field blank from the cycle 3 sampling period was 2.0 percent of its corresponding HHB. For chloride and sulfate, the maximum concentration in any field blank from the cycle 3 sampling period was less than 1 percent of the corresponding SMCL. For fluoride, the maximum concentration was 3.9 percent of the corresponding SMCL. For iron, the maximum concentration in any field blank from the cycle 3 sampling period was 26 percent of its corresponding SMCL of 300 micrograms per liter $(\mu \mathrm{g} / \mathrm{L})$, reported for an August 2016 sample. Results for blind blanks submitted to the NWQL to evaluate laboratory data quality indicate a slight high bias for iron during 2016, but no evidence of a laboratory contamination issue (Tedmund Struzeski, U.S. Geological Survey [USGS] Inorganic Blind Sample Project, written commun., 2017, 2018). Out of 207 NAWQA cycle 3 field blanks for iron, the two results that exceed 10 percent of the SMCL (42.0 and $78.6 \mu \mathrm{g} / \mathrm{L}$ for samples collected in September 2014 and August 2016, respectively) likely reflect isolated events.

Of the 22 trace elements included in laboratory analysis, 20 were detected in at least 1 field blank collected in 2016 (table 4.1), and 21 were detected in at least 1 field blank from the cycle 3 sampling period (table 4.2). Of the 21 trace elements detected in field blanks from the cycle 3 sampling period, 18 (antimony, arsenic, barium, beryllium, boron, cadmium, chromium, copper, lead, manganese, molybdenum, nickel, selenium, silver, strontium, thallium, uranium, and zinc) had HHBs (table 2 of Arnold and others, 2020); and 5 (aluminum, copper, manganese, silver, and zinc) had SMCLs. For 7 of the 18 detected trace elements with HHBs, the maximum concentration measured in a field blank from the cycle 3 sampling period was less than 1 percent of the corresponding HHB; for the remaining 11 trace elements (antimony, arsenic, beryllium, cadmium, copper, lead, manganese, molybdenum, nickel, thallium, and zinc), the maximum concentration was less than 5 percent of the HHB. For silver and zinc, the maximum concentration measured in a field blank from the cycle 3 sampling period was less than 1 percent of the corresponding SMCL; for copper, the maximum concentration was less than 9 percent of the corresponding SMCL. However, for aluminum, the maximum concentration was nearly 200 percent of the corresponding SMCL of $50 \mu \mathrm{g} / \mathrm{L}$. Results for blind blanks submitted to NWQL to evaluate laboratory data quality indicate false positive detections of aluminum in blank samples submitted to the laboratory during July and August 2014, August-December 2015, and June 2016 (USGS Inorganic Blind Sample Project, https://bqs.usgs.gov/ibsp/). Investigation of this issue by the NWQL indicated the occurrence of sporadic contamination at concentrations of as much as $63 \mu \mathrm{g} / \mathrm{L}$ from at least July 2014 through December 2015, although the source of contamination and, therefore, the exact magnitude of contamination and the period(s) affected were not established (Tedmund Struzeski, USGS Inorganic Blind Sample Project, written commun., 2015). For NAWQA field blanks collected through the end of December 2016, reported detections of aluminum above the laboratory reporting limit occurred in blank samples collected primarily between May 29 and July 16, 2014, between March 25 and September 8, 2015, and between April 7 and August 10, 2016.

Also, regarding trace elements, the USGS Office of Water Quality has documented random low-level contamination of water samples with cobalt and manganese from certain capsule filters used by the NAWQA Project and across the USGS from 
about October 1, 2008, to about September 30, 2014 (USGS Office of Water Quality, written commun., July 1, 2016). Blank samples for cobalt and manganese that were collected for NAWQA during this period were evaluated to determine the possible effects of this contamination on environmental samples, and evaluation results were described in appendix 3 of Arnold and others (2017b).

Of the five nutrients or groups of nutrients that the laboratory analyzes directly (as opposed to the nutrients with calculated results), three were detected in at least one field blank collected in 2016, as was dissolved organic carbon (DOC) (table 4.1). All five nutrients and DOC were detected in at least one field blank from the cycle 3 sampling period (table 4.2). Two of the nutrients detected in field blanks from the cycle 3 sampling period (nitrite and nitrate) have HHBs (table 2 of Arnold and others, 2020); none have SMCLs. For each of the two nutrients with HHBs, the maximum concentration measured in a field blank from the cycle 3 sampling period was less than 1 percent of the corresponding threshold. DOC does not have an HHB but was detected in 15 of 41 field blanks collected during 2016, and in 62 of 200 field blanks collected during cycle 3 . Concentrations during cycle 3 ranged from 0.23 to 633 milligrams per liter $(\mathrm{mg} / \mathrm{L})$ and included multiple values above $1 \mathrm{mg} / \mathrm{L}$. However, concentrations of this magnitude probably reflect inadequate rinsing of sampling equipment with blank water between use of methanol during the cleaning process and subsequent collection of the blank sample. Therefore, these results likely are not representative of the actual potential for contamination of environmental samples, which are collected only after flushing of sampling equipment with copious quantities of native groundwater.

Blank samples collected in 2012 were analyzed for 85 VOCs, and blank samples collected in 2013 through 2016 were analyzed for a different (but partially overlapping) list of 85 VOCs; the change in laboratory methods and constituent lists resulted in a total of 129 different VOCs being included in the overall dataset of blank results. In total, 19 VOCs were detected in at least 1 field blank collected in 2016 (table 4.1), and 27 VOCs were detected in at least 1 field blank from the cycle 3 sampling period (table 4.2). Twelve compounds detected in field blanks from the cycle 3 sampling period (1,1-dichloroethene, 1,2-dibromo-3-chloropropane, 1,4-dichlorobenzene, benzene, carbon disulfide, dichloromethane, ethylbenzene, $\mathrm{m}$-xylene plus p-xylene, o-xylene, styrene, toluene, and trichloromethane) have HHBs (table 2 of Arnold and others, 2020) and none have SMCLs. For 9 of the 12 VOCs with HHBs, the maximum concentration measured in a blank was less than 1 percent of the corresponding HHB threshold; for the remaining 3 VOCs (1,1-dichloroethene, 1,2-dibromo3 -chloropropane, and dichloromethane), the maximum concentration was less than 3 percent of the corresponding HHB threshold.

Blank samples collected in 2012 were analyzed for 137 pesticide compounds, and blank samples collected in 2013 through 2016 were analyzed for 225 to 227 pesticide compounds; the change in laboratory methods and constituents resulted in 272 pesticide compounds being included in the overall dataset of blank results. One pesticide compound was detected in 1 field blank collected in 2016 (table 4.1), and 27 pesticide compounds were detected in at least 1 field blank from the cycle 3 sampling period (table 4.2). Eighteen compounds detected in field blanks from the cycle 3 sampling period (aldicarb sulfone, atrazine, bromacil, desulfinylfipronil, diflubenzuron, 2-hydroxy-4-isopropylamino-6-ethylaminos-triazine, imazethapyr, methyoxyfenozide, metolachlor, metribuzin, nicosulfuron, pendimethalin, cis-permethrin, trans-permethrin, piperonyl butoxide, prometryn, propiconazole, and tebuthiuron) have HHBs (table 2 of Arnold and others, 2020) and none have SMCLs. For all the pesticide compounds with HHBs, the maximum concentration measured in a field blank was about 0.25 percent or less of the corresponding HHB threshold.

A few of the special analytes collected in only selected well networks were detected in one or more field blanks from the cycle 3 sampling period. The one field blank collected in 2016 for arsenic speciation had no detections. Six field blanks were collected for arsenic speciation during the cycle 3 sampling period. In these six field blanks, there were no detections of three arsenic species (arsenite, dimethylarsinate, and monomethylarsonate), but arsenate was detected in one field blank at a concentration of $1.51 \mu \mathrm{g} / \mathrm{L}$, which is about 15 percent of the HHB of $10 \mu \mathrm{g} / \mathrm{L}$ for total arsenic. Because it is possible that contamination could limit the suitability of arsenate results for comparison with the arsenic HHB, total arsenic results by NWQL laboratory schedule 2710, which are available for all samples that have arsenate results, should be used for comparison with the HHB. Perchlorate was not detected in any of the 4 field blanks collected during 2016, or in any of the 18 total field blanks collected during the cycle 3 sampling period. Hexavalent chromium was detected in one of the seven field blanks collected in 2015 at a concentration of $0.2 \mu \mathrm{g} / \mathrm{L}$, which is 5.0 percent of the upper cancer HBSL of $4 \mu \mathrm{g} / \mathrm{L}$. Hexavalent chromium was detected in 2 of the 20 field blanks from the cycle 3 sampling period at a maximum concentration of $0.3 \mu \mathrm{g} / \mathrm{L}$, which is 7.5 percent of the upper cancer HBSL of $0.04 \mu \mathrm{g} / \mathrm{L}$. Corresponding total chromium values typically are reported by the same USGS Trace Metal Laboratory in Boulder, Colo., that analyzes for hexavalent chromium. For the four hexavalent chromium field blanks collected in 2016, the corresponding total chromium values were below detection. Of the 19 samples from the cycle 3 sampling period that had total chromium results reported by this laboratory, 2 field blanks had a detection of total chromium at concentrations as much as $0.6 \mu \mathrm{g} / \mathrm{L}$, which is 0.6 percent of the HHB of $100 \mu \mathrm{g} / \mathrm{L}$. Therefore, it seems that there is minimal potential for contamination of total chromium from the USGS Trace Metal Laboratory to affect comparison of these values to HHBs.

The maximum concentrations of nutrients, VOCs, and pesticide compounds in field blanks from the cycle 3 sampling period are all substantially less than the thresholds used by the NAWQA Project to distinguish between low and 
moderate concentrations (50 percent of the HHB or SMCL for inorganic constituents and 10 percent of the HHB for organic constituents); therefore, results of the field blank samples for these constituent groups indicate minimal potential for effects of contamination on the number of groundwater samples that would be classified as having moderate or high concentrations relative to current HHBs or SMCLs. For major, minor, and trace elements, the maximum concentrations in field blanks from the cycle 3 sampling period also are substantially less than the relevant thresholds except for iron and aluminum. Data from third-party blind blanks and from NAWQA Project field blanks do not indicate systematic contamination issues for iron that would substantially affect classification of results relative to the SMCL of $300 \mu \mathrm{g} / \mathrm{L}$. Because data from blind blanks and field blanks indicate that laboratory contamination might have affected aluminum results considerably for some environmental samples from late May 2014 through at least August 2016, aluminum results from this period cannot positively be classified as moderate or high relative to the SMCL of $50 \mu \mathrm{g} / \mathrm{L}$.

\section{Replicate Sample Approach}

Replicate samples are QC samples that are used to estimate variability of analytical results caused by random measurement error (Mueller and others, 2015). Replicate samples are two or more water samples that are collected, processed, and analyzed in a manner that allows them to be considered essentially identical in composition and analysis (Mueller and others, 2015). Replicate groundwater samples for NAWQA consist of two samples collected one after the other in the field (sequential field replicates).

Replicate samples typically are used to evaluate variability in analyte concentration by estimating standard deviation (SD) as a function of concentration (Mueller and others, 2015). The presence of censored values affects the calculation of these estimates and generally necessitates estimation of the variability in analyte detection, as well. One measure of the variability in analyte detection is the mean detection rate for all replicate pairs having at least one detection (Martin, 2002; Mueller and others, 2015). Another measure is the percentage of replicate sets with inconsistent detections, which is calculated as the number of replicate sets with inconsistent detections divided by the total number of replicate sets minus the number of sets with consistent non-detections (Martin, 2002; Mueller and others, 2015). A one-sided upper confidence limit for the percentage of inconsistent replicate sets can be calculated as described by Mueller and others (2015, p. 32). Multiple approaches are available to estimate the variability of analyte concentrations as a function of concentration. Three of these approaches and the requirements to apply them were described by Mueller and others (2015, p. 32).

Data and results of the initial evaluation of data from replicate samples for a variety of analytes collected during the entire cycle 3 sampling period of May 2012 through
December 2016 are presented in this report. The objective of this initial evaluation of replicate samples was to broadly characterize variability in analyte detection and concentration to explore implications for comparisons of environmental concentrations of analytes as reported by the relevant laboratories with their corresponding HHBs or SMCLs. For example, large variability in analyte detection and (or) concentration near an HHB could reduce confidence in the reported concentration representing a true exceedance of an HHB in the environment. As part of this evaluation, for analytes that include censored values and have at least 10 replicate pairs not composed of consistent nondetections, the mean detection rate (for all replicate pairs having at least 1 detection) and the percentage of replicate sets with inconsistent detections were calculated. A piecewise-linear model used by Mueller and Titus (2005) and described by Mueller and others (2015) was used to estimate variability in concentrations for analytes having at least 10 replicate pairs with consistent detections. This two-range model divides concentrations into: (1) a low range for which the SD of replicates generally is uniform and the average SD is used to estimate variability; and (2) a high range for which the relative standard deviation (RSD; the ratio, in percent, of SD to mean concentration) generally is uniform and the average RSD is used to estimate variability. Graphs of SD and RSD against mean concentration are used to select an appropriate boundary concentration between the low and high ranges (Mueller and others, 2015). In some cases, either SD or RSD is fairly uniform throughout the range of available concentrations and, therefore, no boundary is needed.

\section{Replicate Sample Counts}

The total number of replicate samples collected for groundwater sites during cycle 3 varies by analyte group (table 4.3). Data for all replicate samples from the 2015 and 2016 sampling periods are presented in tables 4.10-4.19 of Arnold and others (2020). All cycle 3 replicate samples were analyzed using the corresponding laboratory methods listed in table 2 of Arnold and others (2020). Of the 110 cycle 3 VOC replicate samples, 5 were collected in 2012 and analyzed for an older analytical schedule using purge and trap gas chromatography/mass spectrometry (Gilliom and others, 2006; Zogorski and others, 2006). The remaining 105 were collected in 2013-16 and analyzed using the most recent analytical schedule and laboratory methods (purge and trap gas chromatography/mass spectrometry and heated purge and trap gas chromatography/mass spectrometry). Of the 142 cycle 3 pesticide replicate samples, 4 were collected in 2012 and analyzed for an older analytical schedule using gas chromatography/mass spectrometry. The remaining 138 were collected in 2013-16 and analyzed using the most recent analytical schedule and laboratory method (direct aqueous injection liquid chromatography tandem mass spectrometry). 


\section{Replicate Sample Results}

Analysis of variability in analyte detection, analyte concentration, or both was attempted for only the subset of analytes that met certain requirements. Analysis of replicate results was not attempted for analyte/method combinations for which fewer than 10 replicate pairs had been analyzed. Therefore, VOCs and pesticide compounds determined with older laboratory methods used for analysis of replicates collected in 2012 were not evaluated for variability. In addition, analysis of variability in analyte detection was performed only for analytes that included censored values and had at least 10 replicate pairs without consistent non-detections (table 4.4). Analysis of analyte concentrations was performed only for analytes that had at least 10 replicate pairs with consistent detections (table 4.5).

Among all constituents for which variability in detection was estimated, the mean detection rate ranged from 78.1 to 100 percent and the percentage of pairs with inconsistent detections ranged from 0.0 to 43.8 percent (table 4.4). In his assessment of pesticides, Martin (2002) used a mean detection rate of 75 percent or less or a percentage of inconsistent replicate sets of 50 percent or more to indicate high variability of detection. Using those same criteria, none of the constituents analyzed for this study would be considered to have high variability of detection. Martin (2002) also used a mean detection rate of 90 percent or more or a percentage of inconsistent replicate sets of 25 percent or less to indicate low variability of detection. Under these criteria, the following constituents do not have low variability of detection but rather would be considered to have moderate variability: the nutrient nitrite; the pesticide compounds hexazinone, metolachlor, and OIAT; and the radiological analytes alpha radioactivity (30-day count), radium-226, and radium-228. The initial analysis of variability of detection presented here did not include calculation of an upper confidence bound on percentages of inconsistent replicate sets to quantify uncertainty and did not account for changes in variability across concentration ranges. In general, variability of detection is higher at low concentrations and decreases with increasing concentrations (Martin, 2002). More detailed analysis of this type might be needed for the interpretation of environmental concentrations for objectives other than those presented in this report.

For all constituents for which variability in concentration was estimated, the mean SD, RSD, or both, were determined for specified concentration ranges by means of the two-range model and are presented in table 4.5. For major and minor elements and physical parameters, the mean SD at lower or all concentrations was $0.22 \mathrm{mg} / \mathrm{L}$ or less, except for alkalinity and residue on evaporation (dissolved solids), and the mean RSD at higher concentrations was less than 3 percent, except for bromide and iron. For trace elements, the mean SD at lower or all concentrations was 0.3 microgram per liter or less, except for aluminum and zinc, and the mean RSD at higher concentrations was less than 5 percent, except for cobalt and lead.
For nutrients and dissolved organic carbon, the mean SD at lower concentrations was less than $0.04 \mathrm{mg} / \mathrm{L}$, and the mean RSD at higher concentrations was 3 percent or less, except for dissolved organic carbon. For VOCs, only 4 compounds had at least 10 replicate pairs with consistent detections. Mean SDs were calculated for lower concentration ranges for three compounds and were all 0.013 microgram per liter or less; only one compound had sufficient data to calculate a mean RSD (5.8 percent) for the upper concentration range. For pesticide compounds, 9 compounds had at least 10 replicate pairs with consistent detections. Six compounds had sufficient data to calculate a mean SD for lower concentrations; the mean SD for each of these compounds was less than 10 nanograms per liter, except for metolachlor sulfonic acid. Data for pesticide compounds generally were insufficient to reasonably estimate the mean RSD at higher concentrations. For radiological analytes, the mean SD at lower concentrations was 0.76 picocurie per liter or less, except for radon, and the mean RSD at higher concentrations was less than 10 percent, except for $\alpha$ radioactivity (72-hour count).

None of the special-interest analytes collected in only selected well networks had more than 10 replicate pairs without consistent nondetections for a rigorous evaluation of variability. However, the six available replicate pairs for the four arsenic species indicated no inconsistent detections. Only arsenate and arsenite were detected; within the four to five pairs with consistent detections of these analytes, concentrations were similar. Out of 10 sample pairs, perchlorate was not detected in either sample of 3 pairs, was detected in 1 sample of a single pair (at a concentration nearly equivalent to the laboratory reporting level) and was detected in both samples of 6 pairs (at similar concentrations). Out of 9 samples pairs, hexavalent chromium was not detected in either sample of 3 pairs and had inconsistent detections in 3 of the 6 other pairs; concentrations within the 3 pairs with consistent detections were similar. Out of 8 sample pairs for total chromium from the Boulder, Colo., laboratory, chromium was not detected in either sample of 2 pairs, was detected in 1 sample of 2 pairs, and was detected in both samples of 4 pairs ( 2 of which had similar concentrations between samples). Four replicate samples were analyzed for strontium isotope ratios, for which a non-detection is not a possible result; the isotope ratios within all four sample pairs were identical.

Overall, the available results indicate generally low variability in analyte detection and concentration, meaning that random measurement error has minimal potential to affect the number of groundwater samples that would be classified as having moderate or high concentrations relative to current HHBs or SMCLs. However, further analysis beyond the scope of this initial evaluation of replicate results, such as the calculation of confidence intervals, would be needed to quantify the likely effects of variability for use of the environmental data for specific purposes. 
Table 4.4. Estimated variability in detection of selected analytes based on field replicate samples collected by the U.S. Geological Survey National Water-Quality Assessment Project, May 2013 through December 2016.

[Variability was evaluated only for constituents having censored values and at least 10 replicate pairs without consistent nondetections. $n$, number of pairs with at least one detection; CAAT, chlorodiamino-s-triazine; CEAT, 2-chloro-6-ethylamino-4-amino-s-triazine; OIAT, 2-hydroxy-4-isopropylamino-6-amino-striazine; OIET, 2-hydroxy-4-isopropylamino-6-ethylamino-s-triazine; SA, sulfonic acid]

\begin{tabular}{|c|c|c|c|}
\hline Constituent & $n$ & $\begin{array}{l}\text { Mean detection rate } \\
\text { (percent) }\end{array}$ & Pairs with inconsistent detections (percent) \\
\hline \multicolumn{4}{|c|}{ Major and minor elements } \\
\hline Bromide & 95 & 97.4 & 5.3 \\
\hline Fluoride & 113 & 100 & 0 \\
\hline Dissolved solids & 118 & 99.6 & 0.8 \\
\hline Iron & 80 & 90.6 & 18.8 \\
\hline Sulfate & 115 & 100 & 0 \\
\hline \multicolumn{4}{|c|}{ Trace elements } \\
\hline Aluminum & 52 & 90.4 & 19.2 \\
\hline Antimony & 50 & 95 & 10 \\
\hline Arsenic & 102 & 98.5 & 2.9 \\
\hline Beryllium & 30 & 100 & 0 \\
\hline Boron & 111 & 99.5 & 0.9 \\
\hline Cadmium & 28 & 94.6 & 10.7 \\
\hline Chromium & 54 & 97.2 & 5.6 \\
\hline Cobalt & 82 & 94.5 & 11 \\
\hline Copper & 54 & 89.8 & 20.4 \\
\hline Lead & 81 & 92.6 & 14.8 \\
\hline Lithium & 118 & 100 & 0 \\
\hline Manganese & 92 & 97.8 & 4.3 \\
\hline Molybdenum & 107 & 100 & 0 \\
\hline Nickel & 94 & 98.9 & 2.1 \\
\hline Selenium & 85 & 96.5 & 7.1 \\
\hline Thallium & 16 & 96.9 & 6.3 \\
\hline Uranium & 102 & 99 & 2 \\
\hline Vanadium & 99 & 96 & 8.1 \\
\hline Zinc & 66 & 94.7 & 10.6 \\
\hline \multicolumn{4}{|c|}{ Nutrients and dissolved organic carbon } \\
\hline Ammonia & 50 & 93 & 14 \\
\hline Nitrite plus nitrate & 77 & 98.7 & 2.6 \\
\hline Nitrite & 26 & 84.6 & 30.8 \\
\hline Total nitrogen & 108 & 99.5 & 0.9 \\
\hline Orthophosphate & 106 & 99.5 & 0.9 \\
\hline Dissolved organic carbon & 80 & 95.6 & 8.8 \\
\hline
\end{tabular}


Table 4.4. Estimated variability in detection of selected analytes based on field replicate samples collected by the U.S. Geological Survey National Water-Quality Assessment Project, May 2013 through December 2016.—Continued

[Variability was evaluated only for constituents having censored values and at least 10 replicate pairs without consistent nondetections. $n$, number of pairs with at least one detection; CAAT, chlorodiamino-s-triazine; CEAT, 2-chloro-6-ethylamino-4-amino-s-triazine; OIAT, 2-hydroxy-4-isopropylamino-6-amino-striazine; OIET, 2-hydroxy-4-isopropylamino-6-ethylamino-s-triazine; SA, sulfonic acid]

\begin{tabular}{|c|c|c|c|}
\hline Constituent & $n$ & $\begin{array}{l}\text { Mean detection rate } \\
\text { (percent) }\end{array}$ & Pairs with inconsistent detections (percent) \\
\hline \multicolumn{4}{|c|}{ Volatile organic compounds } \\
\hline 1,2-Dichloropropane & 11 & 100 & 0 \\
\hline Carbon disulfide & 16 & 93.8 & 12.5 \\
\hline Methyl tert-butyl ether & 17 & 100 & 0 \\
\hline Trichloromethane & 28 & 100 & 0 \\
\hline \multicolumn{4}{|c|}{ Pesticide compounds } \\
\hline Atrazine & 39 & 91 & 17.9 \\
\hline CAAT & 41 & 96.3 & 7.3 \\
\hline CEAT & 23 & 97.8 & 4.3 \\
\hline Deethylatrazine (CIAT) & 41 & 89 & 22 \\
\hline Hexazinone & 16 & 81.3 & 37.5 \\
\hline Metolachlor & 16 & 78.1 & 43.8 \\
\hline Metolachlor SA & 43 & 95.3 & 9.3 \\
\hline OIAT & 15 & 80 & 40 \\
\hline OIET & 12 & 95.8 & 8.3 \\
\hline Prometon & 13 & 92.3 & 15.4 \\
\hline Simazine & 18 & 91.7 & 16.7 \\
\hline \multicolumn{4}{|c|}{ Radiological analytes } \\
\hline$\alpha$ radioactivity, 30 -day count & 57 & 85.1 & 29.8 \\
\hline$\alpha$ radioactivity, 72 -hour count & 68 & 89 & 22.1 \\
\hline$\beta$ radioactivity, 30-day count & 76 & 93.4 & 13.2 \\
\hline$\beta$ radioactivity, 72-hour count & 79 & 94.3 & 11.4 \\
\hline Radium-224 & 59 & 89.8 & 20.3 \\
\hline Radium-226 & 74 & 81.8 & 36.5 \\
\hline Radium-228 & 54 & 84.3 & 31.5 \\
\hline
\end{tabular}

\section{Spike Sample Approach}

Spike samples are QC samples that are used to estimate any positive or negative bias that might result from method performance, effects of the sample matrix, and (or) analyte degradation during sample shipment and storage (Mueller and others, 2015). Spike samples are collected by fortifying (spiking) a water sample with known concentrations of analytes. For VOCs, NAWQA collects laboratory matrix spikes, meaning that the spike solution is added to an environmental sample at the laboratory. For pesticide compounds and arsenic species, NAWQA collects field matrix spikes, meaning that the spike solution is added to an environmental sample in the field. Both types of spikes estimate recovery bias in an environmental water sample that could be caused by a problem with performance of the laboratory method, by the chemical, physical, or biological characteristics of the water, or by both (Mueller and others, 2015). Field spikes also reflect any degradation that might have occurred in an analyte during the time between sample collection and laboratory analysis. Evaluations of recoveries for laboratory spikes for VOCs and field spikes for arsenic species are included in this report, but an evaluation of recoveries for field spikes for pesticides is not included here because Shoda and others (2018) presented this type of evaluation for pesticide spike samples collected for the NAWQA Project in 2013-15. 
Table 4.5. Estimated variability in concentrations of selected analytes based on field replicate samples collected by the U.S. Geological Survey National Water-Quality Assessment Project, May 2013 through December 2016.

[Variability was evaluated only for constituents having at least 10 replicate pairs with consistent detections. $n$, number of values in that category; IPT, inflection point titration method; $\mathrm{mg} / \mathrm{L}$, milligram per liter; $\mathrm{CaCO}_{3}$, calcium carbonate; $\mu \mathrm{S} / \mathrm{cm}$, microsiemen per centimeter at $25 \mathrm{degrees} \mathrm{Celsius;} \mu \mathrm{g} / \mathrm{L}$, microgram per liter; InsD, insufficient data; ng/L, nanogram per liter; SD, standard deviation; RSD, relative standard deviation; CAAT, chlorodiamino-s-triazine; CEAT, 2-chloro-6-ethylamino-4-amino-s-triazine; OIET, 2-hydroxy-4-isopropylamino-6-ethylamino-s-triazine; SA, sulfonic acid; pCi/L, picocurie per liter]

\begin{tabular}{|c|c|c|c|c|c|}
\hline \multirow{2}{*}{ Constituent } & \multirow{2}{*}{ Concentration range (unit) } & \multirow{2}{*}{$n$} & \multicolumn{3}{|c|}{ Variability } \\
\hline & & & Statistic & Value & Unit \\
\hline \multicolumn{6}{|c|}{ Major and minor elements and physical parameters } \\
\hline Alkalinity by IPT & $\begin{array}{l}\text { All available values } \\
\left(\mathrm{mg} / \mathrm{L} \text { as } \mathrm{CaCO}_{3}\right)\end{array}$ & 58 & Mean SD & 6.4 & $\mathrm{mg} / \mathrm{L}$ as $\mathrm{CaCO}_{3}$ \\
\hline $\mathrm{pH}$ & All available values ( $\mathrm{pH}$ units) & 118 & Mean SD & 0.031 & $\mathrm{pH}$ units \\
\hline Specific conductance & All available values $(\mu \mathrm{S} / \mathrm{cm})$ & 118 & Mean RSD & 0.24 & percent \\
\hline \multirow[t]{2}{*}{ Calcium } & $<10$ & 23 & Mean SD & 0.059 & $\mathrm{mg} / \mathrm{L}$ \\
\hline & $>10$ & 96 & Mean RSD & 1.3 & percent \\
\hline Magnesium & All available concentrations & 119 & Mean RSD & 1 & percent \\
\hline \multirow[t]{2}{*}{ Potassium } & $<1.5$ & 45 & Mean SD & 0.016 & $\mathrm{mg} / \mathrm{L}$ \\
\hline & $>1.5$ & 74 & Mean RSD & 1.9 & percent \\
\hline Sodium & All available concentrations & 119 & Mean RSD & 1.5 & percent \\
\hline \multirow[t]{2}{*}{ Bromide } & $<0.1$ & 53 & Mean SD & 0.0026 & $\mathrm{mg} / \mathrm{L}$ \\
\hline & $>0.1$ & 37 & Mean RSD & 5.7 & percent \\
\hline \multirow[t]{2}{*}{ Chloride } & $<40$ & 91 & Mean SD & 0.05 & $\mathrm{mg} / \mathrm{L}$ \\
\hline & $>40$ & 28 & Mean RSD & 1.2 & percent \\
\hline \multirow[t]{2}{*}{ Fluoride } & $<0.2$ & 62 & Mean SD & 0.0014 & $\mathrm{mg} / \mathrm{L}$ \\
\hline & $>0.2$ & 51 & Mean RSD & 2.5 & percent \\
\hline Silica & All available concentrations & 119 & Mean SD & 0.22 & $\mathrm{mg} / \mathrm{L}$ \\
\hline \multirow[t]{2}{*}{ Sulfate } & $<40$ & 72 & Mean SD & 0.029 & $\mathrm{mg} / \mathrm{L}$ \\
\hline & $>40$ & 43 & Mean RSD & 0.93 & percent \\
\hline \multirow[t]{2}{*}{ Residue on evaporation } & $<900$ & 107 & Mean SD & 7.3 & $\mathrm{mg} / \mathrm{L}$ \\
\hline & $>900$ & 10 & Mean RSD & 0.82 & percent \\
\hline \multirow[t]{2}{*}{ Iron } & $<100$ & 36 & Mean SD & 5.3 & $\mu \mathrm{g} / \mathrm{L}$ \\
\hline & $>100$ & 29 & Mean RSD & 5.4 & percent \\
\hline \multicolumn{6}{|c|}{ Trace elements } \\
\hline Aluminum & All available concentrations & 42 & Mean SD & 1 & $\mu \mathrm{g} / \mathrm{L}$ \\
\hline \multirow[t]{2}{*}{ Antimony } & $<0.1$ & 33 & Mean SD & 0.0026 & $\mu \mathrm{g} / \mathrm{L}$ \\
\hline & $>0.1$ & 12 & Mean RSD & 4.3 & percent \\
\hline \multirow[t]{2}{*}{ Arsenic } & $<1.0$ & 48 & Mean SD & 0.0083 & $\mu \mathrm{g} / \mathrm{L}$ \\
\hline & $>1.0$ & 51 & Mean RSD & 1.6 & percent \\
\hline \multirow[t]{2}{*}{ Barium } & $<50$ & 73 & Mean SD & 0.22 & $\mu \mathrm{g} / \mathrm{L}$ \\
\hline & $>50$ & 47 & Mean RSD & 1.6 & percent \\
\hline Beryllium & All available concentrations & 30 & Mean SD & 0.0028 & $\mu \mathrm{g} / \mathrm{L}$ \\
\hline \multirow[t]{2}{*}{ Boron } & $<30$ & 44 & Mean SD & 0.3 & $\mu \mathrm{g} / \mathrm{L}$ \\
\hline & $>30$ & 66 & Mean RSD & 1.9 & percent \\
\hline Cadmium & All available concentrations & 25 & Mean SD & 0.0036 & $\mu \mathrm{g} / \mathrm{L}$ \\
\hline \multirow[t]{2}{*}{ Chromium } & $<2$ & 39 & Mean SD & 0.028 & $\mu \mathrm{g} / \mathrm{L}$ \\
\hline & $>2$ & 12 & Mean RSD & 2.6 & percent \\
\hline
\end{tabular}


Table 4.5. Estimated variability in concentrations of selected analytes based on field replicate samples collected by the U.S. Geological Survey National Water-Quality Assessment Project, May 2013 through December 2016.—Continued

[Variability was evaluated only for constituents having at least 10 replicate pairs with consistent detections. $n$, number of values in that category; IPT, inflection point titration method; $\mathrm{mg} / \mathrm{L}$, milligram per liter; $\mathrm{CaCO}_{3}$, calcium carbonate; $\mu \mathrm{S} / \mathrm{cm}$, microsiemen per centimeter at $25 \mathrm{degrees} \mathrm{Celsius;} \mu \mathrm{g} / \mathrm{L}$, microgram per liter; InsD, insufficient data; ng/L, nanogram per liter; SD, standard deviation; RSD, relative standard deviation; CAAT, chlorodiamino-s-triazine; CEAT, 2-chloro-6-ethylamino-4-amino-s-triazine; OIET, 2-hydroxy-4-isopropylamino-6-ethylamino-s-triazine; SA, sulfonic acid; pCi/L, picocurie per liter]

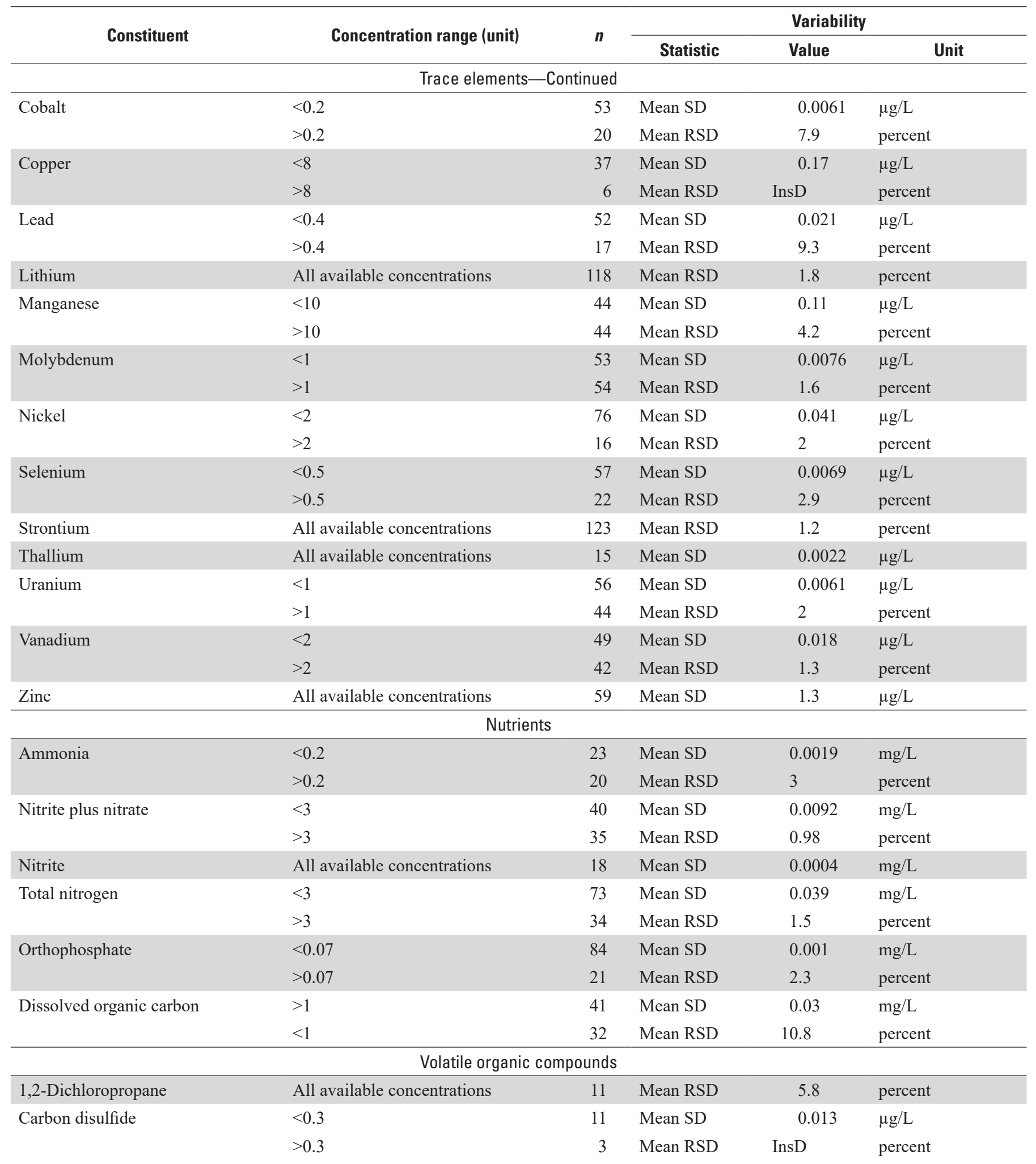


Table 4.5. Estimated variability in concentrations of selected analytes based on field replicate samples collected by the U.S. Geological Survey National Water-Quality Assessment Project, May 2013 through December 2016.—Continued

[Variability was evaluated only for constituents having at least 10 replicate pairs with consistent detections. $n$, number of values in that category; IPT, inflection point titration method; $\mathrm{mg} / \mathrm{L}$, milligram per liter; $\mathrm{CaCO}_{3}$, calcium carbonate; $\mu \mathrm{S} / \mathrm{cm}$, microsiemen per centimeter at $25 \mathrm{degrees} \mathrm{Celsius;} \mu \mathrm{g} / \mathrm{L}$, microgram per liter; InsD, insufficient data; ng/L, nanogram per liter; SD, standard deviation; RSD, relative standard deviation; CAAT, chlorodiamino-s-triazine; CEAT, 2-chloro-6-ethylamino-4-amino-s-triazine; OIET, 2-hydroxy-4-isopropylamino-6-ethylamino-s-triazine; SA, sulfonic acid; pCi/L, picocurie per liter]

\begin{tabular}{|c|c|c|c|c|c|}
\hline \multirow{2}{*}{ Constituent } & \multirow{2}{*}{ Concentration range (unit) } & \multirow{2}{*}{$n$} & \multicolumn{3}{|c|}{ Variability } \\
\hline & & & Statistic & Value & Unit \\
\hline \multicolumn{6}{|c|}{ Volatile organic compounds_-Continued } \\
\hline \multirow[t]{2}{*}{ Methyl tert-butyl ether } & $<0.3$ & 14 & Mean SD & 0.0014 & $\mu \mathrm{g} / \mathrm{L}$ \\
\hline & $>0.3$ & 3 & Mean RSD & InsD & percent \\
\hline \multirow[t]{2}{*}{ Trichloromethane } & $<0.2$ & 20 & Mean SD & 0.0013 & $\mu \mathrm{g} / \mathrm{L}$ \\
\hline & $>0.2$ & 9 & Mean RSD & InsD & percent \\
\hline \multicolumn{6}{|c|}{ Pesticide compounds } \\
\hline \multirow[t]{2}{*}{ Atrazine } & $<90$ & 29 & Mean SD & 1.1 & $\mathrm{ng} / \mathrm{L}$ \\
\hline & $>90$ & 3 & Mean RSD & InsD & percent \\
\hline \multirow[t]{2}{*}{ Deethylatrazine (CIAT) } & $<200$ & 27 & Mean SD & 4.8 & $\mathrm{ng} / \mathrm{L}$ \\
\hline & $>200$ & 5 & Mean RSD & InsD & percent \\
\hline \multirow[t]{2}{*}{ CEAT } & $<500$ & 21 & Mean SD & 7.7 & $\mathrm{ng} / \mathrm{L}$ \\
\hline & $>500$ & 1 & Mean RSD & InsD & percent \\
\hline \multirow[t]{2}{*}{ CAAT } & $<65$ & 12 & Mean SD & 4 & $\mathrm{ng} / \mathrm{L}$ \\
\hline & $>65$ & 26 & Mean RSD & 13 & percent \\
\hline \multirow[t]{2}{*}{ Hexazinone } & $<15$ & 8 & Mean SD & InsD & $\mathrm{ng} / \mathrm{L}$ \\
\hline & $>15$ & 2 & Mean RSD & InsD & percent \\
\hline \multirow[t]{2}{*}{ Metolachlor SA } & $<500$ & 14 & Mean SD & 19 & $\mathrm{ng} / \mathrm{L}$ \\
\hline & $>500$ & 5 & Mean RSD & 3.6 & percent \\
\hline \multirow[t]{2}{*}{ OIET } & $<50$ & 9 & Mean SD & InsD & $\mathrm{ng} / \mathrm{L}$ \\
\hline & $>50$ & 2 & Mean RSD & InsD & percent \\
\hline \multirow[t]{2}{*}{ Prometon } & $<10$ & 6 & Mean SD & InsD & $\mathrm{ng} / \mathrm{L}$ \\
\hline & $>10$ & 5 & Mean RSD & InsD & percent \\
\hline \multirow[t]{2}{*}{ Simazine } & $<50$ & 14 & Mean SD & 2.1 & $\mathrm{ng} / \mathrm{L}$ \\
\hline & $>50$ & 1 & Mean RSD & InsD & percent \\
\hline \multicolumn{6}{|c|}{ Radiological analytes } \\
\hline \multirow[t]{2}{*}{$\alpha$ radioactivity, 30 -day count } & $<5$ & 25 & Mean SD & 0.5 & $\mathrm{pCi} / \mathrm{L}$ \\
\hline & $>5$ & 15 & Mean RSD & 9.7 & percent \\
\hline \multirow[t]{2}{*}{$\alpha$ radioactivity, 72 -hour count } & $<10$ & 41 & Mean SD & 0.55 & $\mathrm{pCi} / \mathrm{L}$ \\
\hline & $>10$ & 12 & Mean RSD & 14 & percent \\
\hline \multirow[t]{2}{*}{$\beta$ radioactivity, 30-day count } & $<8$ & 44 & Mean SD & 0.59 & $\mathrm{pCi} / \mathrm{L}$ \\
\hline & $>8$ & 22 & Mean RSD & 8.3 & percent \\
\hline \multirow[t]{2}{*}{$\beta$ radioactivity, 72-hour count } & $<10$ & 57 & Mean SD & 0.76 & $\mathrm{pCi} / \mathrm{L}$ \\
\hline & $\geq 10$ & 13 & Mean RSD & 8.6 & percent \\
\hline Radium-224 & All available concentrations & 48 & Mean SD & 0.15 & $\mathrm{pCi} / \mathrm{L}$ \\
\hline Radium-226 & All available concentrations & 48 & Mean SD & 0.13 & $\mathrm{pCi} / \mathrm{L}$ \\
\hline Radium-228 & All available concentrations & 38 & Mean SD & 0.16 & $\mathrm{pCi} / \mathrm{L}$ \\
\hline \multirow[t]{2}{*}{ Radon } & $<500$ & 32 & Mean SD & 12 & $\mathrm{pCi} / \mathrm{L}$ \\
\hline & $>500$ & 14 & Mean RSD & 2.9 & percent \\
\hline
\end{tabular}


The percent recovery of an analyte in an individual spike sample is calculated by subtracting the concentration of the paired unspiked sample (collected closely in time) from the concentration of the spiked sample, then dividing by the expected concentration and multiplying by 100 . The expected concentration is equal to the concentration of the spike solution times the amount of spike solution added to the sample, divided by the volume of the spiked sample. When the NWQL reported that an analyte was not detected in the paired unspiked sample, a concentration of zero was assumed for the purposes of the calculating the percent recovery. Spike solutions were obtained from the NWQL, which provides the concentration of each analyte included in an individual spike lot. Analytes included in spike solutions were assumed not to have degraded before use of the spike solution, although it is possible that future evaluation of spike sample results might indicate that the assumption is violated for certain spike lots, compounds, or both. For VOCs, the NWQL was assumed to have added 20 microliters of spike solution to a 43-milliliter sample. For arsenic species, field crews were assumed to have added 100 microliters of spike solution to an 11.5-milliliter sample. Samples were excluded from analysis of recoveries when there was evidence that they had been collected after chlorination, which can affect the analysis of many compounds. Individual results were excluded from analysis of recoveries when the concentration present for the compound in the unspiked sample exceeded the expected spike concentration because this can result in increased uncertainty in recovery (Shoda and others, 2018).

Results of an initial evaluation of recovery data from laboratory spikes for VOCs analyzed under laboratory schedules 4436 (S4436) and 4437 (S4437) and from field spikes for arsenic species analyzed under laboratory code 3142 (LC3142) during the entire cycle 3 sampling period are presented in this report (laboratory schedules and codes are listed in table 2 of Arnold and others (2020, 2017b, 2018b). Data for laboratory spikes collected for VOCs and field spikes collected for arsenic species in January 2015-December 2016 also are published in this report. Data and results of earlier evaluations of recovery data from laboratory spikes collected for VOCs in 2012 under older laboratory schedules 2020 and 4024 and from laboratory spikes collected for VOCs collected in May 2013 through December 2014 for S4436 and S4437 are presented in Arnold and others (2017a,b); data and results of an earlier evaluation of recovery data for field spikes collected for arsenic speciation in 2014 also are presented there. Although an evaluation of recovery results for field spikes collected for pesticides is not included in this report, data for pesticide spike samples collected in May 2013-December 2016 are published here.

The objective of performing an initial evaluation of spike samples was to determine if substantial positive or negative recovery bias exists for any analytes. Substantial positive or negative bias could have implications for comparisons of environmental concentrations of these analytes as reported by the NWQL with their corresponding HHBs. For example, a large negative recovery bias could result in the laboratory reporting a concentration that is substantially less than the concentration actually present in the environment, leading to an incorrect conclusion that the concentration in the environment does not exceed an HHB when it actually does. For the purposes of this initial evaluation of spike samples, a median recovery between 70 and 130 percent is considered acceptable. Further evaluation of results for spike samples would be needed to determine if recovery bias for certain analytes would affect the interpretation of environmental concentrations of those analytes for objectives other than those presented in this report.

\section{Spike Sample Counts}

Between May 2013 and December 2016, a total of 96 laboratory spikes for VOCs by S4436, 97 laboratory spikes for VOCs by S4437, and 9 field spikes for arsenic species were collected for the NAWQA Project (tables 4.6-4.9). Data for VOC and arsenic species spike samples from January 2015 through December 2016 are presented in tables 4.13-4.15 of Arnold and others (2020). Data for the 60 field spikes collected for pesticides by S2437 during cycle 3 are presented in table 4.14 of Arnold and others (2020).

\section{Spike Sample Results}

For VOC laboratory spikes collected during cycle 3 for analysis by $\mathrm{S} 4436$ or $\mathrm{S} 4437$, the median recovery for individual compounds ranged from 69.4 to 110.4 percent (tables 4.6-4.8; figs. 4.1 and 4.2; table 4.13 of Arnold and others, 2020). Only butane, which is included on S4436 and has no HHB, had a median recovery less than 70 percent. No VOCs had median recoveries greater than 130 percent. Therefore, laboratory spike recovery results do not indicate any issues with comparing reported VOC concentrations to their corresponding HHBs.

For the nine arsenic speciation field spikes collected during cycle 3 for analysis by LC 3142 , recoveries ranged from 77.3 to 124.7 percent for arsenate, 60.6 to 111.2 percent for arsenite, 63.6 to 100.0 percent for dimethylarsinate, and 70.6 to 103.3 percent for monomethylarsonate (table 4.15 of Arnold and others, 2020; fig. 4.3). Median recoveries for all four species were between 85.3 and 90.6 percent (table 4.6). These results indicate bias from method performance, effects of the sample matrix, or analyte degradation, or all of the above generally is small but is more likely to be slightly negative than positive. No HHBs exist that are specific to individual arsenic species, although there is an HHB for total arsenic. 
Table 4.6. Summary of results for spike samples collected by the National Water-Quality Assessment Project from May 2013 to December 2016.

[VOC, volatile organic compound; HHB, human-health benchmark; --, not applicable]

\begin{tabular}{lccc}
\hline \multicolumn{1}{c}{ Type of summary } & V0C by schedule & V0C by schedule & Arsenic species \\
\hline Total number of field spikes & $\mathbf{4 4 3 6}$ & $\mathbf{4 4 3 7}$ & 9 \\
Total number of laboratory blanks & 0 & 0 & 0 \\
Number of constituents analyzed & 96 & 97 & 4 \\
Range of median spike recoveries, in percent & 49 & 38 & $85.3-90.6$ \\
$\quad$ Number of compounds with median spike recovery less than & 1 & 0 & 0 \\
$\quad 70$ percent & $0.4-107.5$ & -- & -- \\
$\begin{array}{l}\text { Number of compounds with median spike recovery less than } \\
70 \text { percent with a corresponding HHB }\end{array}$ & 0 & 0 & 0 \\
$\begin{array}{l}\text { Number of compounds with median spike recovery greater } \\
\text { than 130 percent }\end{array}$ & 0 & -- & -- \\
$\quad \begin{array}{l}\text { Number of compounds with median spike recovery greater } \\
\text { than 130 percent with a corresponding HHB }\end{array}$ & -- & & \\
\hline
\end{tabular}


Table 4.7. Statistical summary of laboratory spike recovery results for volatile organic compounds included in laboratory schedule 4436.

[n, number of values for that constituent; min, minimum; 10th, 25th, 75th, and 90th, 10th, 25th, 75th, and 90th percentiles; med, median, max, maximum]

\begin{tabular}{|c|c|c|c|c|c|c|c|c|c|}
\hline \multirow{2}{*}{$\begin{array}{l}\text { Parameter } \\
\text { code }\end{array}$} & \multirow{2}{*}{ Constituent } & \multicolumn{8}{|c|}{ Statistic } \\
\hline & & $n$ & Min & 10th & 25th & Med & 75th & 90th & Max \\
\hline P62174 & $\begin{array}{l}\text { 2,2-Dichloro-1,1,1-trifluoroethane, water, unfil- } \\
\text { tered, recoverable, micrograms per liter }\end{array}$ & 96 & 84.3 & 94.1 & 98.7 & 101.7 & 107.4 & 110.9 & 118.6 \\
\hline P45028 & $\begin{array}{l}\text { Chlorodifluoromethane, water, unfiltered, recov- } \\
\text { erable, micrograms per liter }\end{array}$ & 96 & 67.4 & 79.0 & 87.1 & 94.0 & 105.6 & 117.2 & 133.0 \\
\hline P77119 & $\begin{array}{l}\text { Dichlorofluoromethane, water, unfiltered, recov- } \\
\text { erable, micrograms per liter }\end{array}$ & 96 & 90.2 & 95.1 & 100.6 & 103.1 & 108.5 & 114.9 & 124.2 \\
\hline P81590 & $\begin{array}{l}\text { Hexane, water, unfiltered, recoverable, micro- } \\
\text { grams per liter }\end{array}$ & 96 & 42.3 & 55.4 & 64.5 & 75.1 & 83.2 & 92.1 & 103.8 \\
\hline P81604 & $\begin{array}{l}n \text {-Pentane, water, unfiltered, recoverable, micro- } \\
\text { grams per liter }\end{array}$ & 96 & 55.2 & 65.2 & 76.3 & 88.8 & 98.6 & 106.9 & 124.7 \\
\hline P77562 & $\begin{array}{l}\text { 1,1,1,2-Tetrachloroethane, water, unfiltered, } \\
\text { recoverable, micrograms per liter }\end{array}$ & 96 & 78.8 & 88.4 & 90.8 & 95.3 & 100.7 & 106.9 & 120.3 \\
\hline P34506 & $\begin{array}{l}\text { 1,1,1-Trichloroethane, water, unfiltered, recov- } \\
\text { erable, micrograms per liter }\end{array}$ & 96 & 81.9 & 91.8 & 96.2 & 100.6 & 105.5 & 107.7 & 122.2 \\
\hline P34511 & $\begin{array}{l}\text { 1,1,2-Trichloroethane, water, unfiltered, recov- } \\
\text { erable, micrograms per liter }\end{array}$ & 96 & 86.3 & 91.8 & 96.9 & 100.7 & 104.7 & 109.8 & 118.9 \\
\hline P34496 & $\begin{array}{l}\text { 1,1-Dichloroethane, water, unfiltered, recover- } \\
\text { able, micrograms per liter }\end{array}$ & 96 & 82.7 & 93.3 & 98.0 & 101.3 & 106.1 & 110.2 & 124.2 \\
\hline P34501 & $\begin{array}{l}\text { 1,1-Dichloroethene, water, unfiltered, recover- } \\
\text { able, micrograms per liter }\end{array}$ & 96 & 81.0 & 86.3 & 91.8 & 99.4 & 103.3 & 108.5 & 117.7 \\
\hline P34551 & $\begin{array}{l}\text { 1,2,4-Trichlorobenzene, water, unfiltered, } \\
\text { recoverable, micrograms per liter }\end{array}$ & 96 & 75.4 & 84.8 & 88.1 & 92.2 & 98.2 & 104.7 & 116.5 \\
\hline P77222 & $\begin{array}{l}\text { 1,2,4-Trimethylbenzene, water, unfiltered, } \\
\text { recoverable, micrograms per liter }\end{array}$ & 96 & 73.5 & 80.5 & 84.5 & 95.8 & 107.0 & 117.3 & 133.0 \\
\hline P34536 & $\begin{array}{l}\text { 1,2-Dichlorobenzene, water, unfiltered, recover- } \\
\text { able, micrograms per liter }\end{array}$ & 96 & 83.7 & 99.8 & 103.5 & 107.5 & 111.5 & 115.9 & 124.5 \\
\hline P32103 & $\begin{array}{l}\text { 1,2-Dichloroethane, water, unfiltered, recover- } \\
\text { able, micrograms per liter }\end{array}$ & 96 & 87.4 & 95.5 & 97.9 & 100.5 & 103.2 & 107.2 & 115.9 \\
\hline P34571 & $\begin{array}{l}\text { 1,4-Dichlorobenzene, water, unfiltered, recover- } \\
\text { able, micrograms per liter }\end{array}$ & 96 & 84.1 & 93.9 & 97.6 & 101.6 & 107.1 & 112.6 & 126.1 \\
\hline P34030 & $\begin{array}{l}\text { Benzene, water, unfiltered, recoverable, micro- } \\
\text { grams per liter }\end{array}$ & 96 & 83.5 & 90.8 & 97.9 & 101.3 & 106.1 & 110.2 & 123.9 \\
\hline P77297 & $\begin{array}{l}\text { Bromochloromethane, water, unfiltered, recov- } \\
\text { erable, micrograms per liter }\end{array}$ & 96 & 86.2 & 95.2 & 99.9 & 103.7 & 109.5 & 114.4 & 121.6 \\
\hline P32101 & $\begin{array}{l}\text { Bromodichloromethane, water, unfiltered, } \\
\text { recoverable, micrograms per liter }\end{array}$ & 96 & 79.7 & 85.6 & 89.1 & 94.3 & 104.3 & 110.9 & 127.0 \\
\hline P34413 & $\begin{array}{l}\text { Bromomethane, water, unfiltered, recoverable, } \\
\text { micrograms per liter }\end{array}$ & 96 & 42.6 & 82.8 & 95.3 & 107.0 & 125.8 & 149.6 & 195.6 \\
\hline P77041 & $\begin{array}{l}\text { Carbon disulfide, water, unfiltered, micrograms } \\
\text { per liter }\end{array}$ & 93 & 42.4 & 73.2 & 78.9 & 88.7 & 97.8 & 110.0 & 175.5 \\
\hline P34301 & $\begin{array}{l}\text { Chlorobenzene, water, unfiltered, recoverable, } \\
\text { micrograms per liter }\end{array}$ & 96 & 80.9 & 85.6 & 89.0 & 93.4 & 100.4 & 105.2 & 116.3 \\
\hline P34418 & $\begin{array}{l}\text { Chloromethane, water, unfiltered, recoverable, } \\
\text { micrograms per liter }\end{array}$ & 96 & 56.0 & 74.8 & 89.1 & 102.6 & 113.9 & 129.8 & 154.8 \\
\hline P77093 & $\begin{array}{l}\text { cis-1,2-Dichloroethene, water, unfiltered, recov- } \\
\text { erable, micrograms per liter }\end{array}$ & 96 & 84.4 & 92.0 & 94.8 & 99.0 & 102.6 & 110.1 & 121.1 \\
\hline
\end{tabular}


Table 4.7. Statistical summary of laboratory spike recovery results for volatile organic compounds included in laboratory schedule 4436.-Continued

[n, number of values for that constituent; min, minimum; 10th, 25th, 75th, and 90th, 10th, 25th, 75th, and 90th percentiles; med, median, max, maximum]

\begin{tabular}{|c|c|c|c|c|c|c|c|c|c|}
\hline \multirow{2}{*}{$\begin{array}{l}\text { Parameter } \\
\text { code }\end{array}$} & \multirow{2}{*}{ Constituent } & \multicolumn{8}{|c|}{ Statistic } \\
\hline & & $n$ & Min & 10th & 25th & Med & 75th & 90th & Max \\
\hline P34704 & $\begin{array}{l}\text { cis-1,3-Dichloropropene, water, unfiltered, } \\
\text { recoverable, micrograms per liter }\end{array}$ & 96 & 78.3 & 84.9 & 88.3 & 92.1 & 98.4 & 104.4 & 115.3 \\
\hline P32105 & $\begin{array}{l}\text { Dibromochloromethane, water, unfiltered, } \\
\text { recoverable, micrograms per liter }\end{array}$ & 96 & 80.8 & 83.4 & 87.1 & 92.3 & 102.1 & 107.2 & 127.8 \\
\hline P34423 & $\begin{array}{l}\text { Dichloromethane, water, unfiltered, recoverable, } \\
\text { micrograms per liter }\end{array}$ & 96 & 74.3 & 93.9 & 98.6 & 101.6 & 105.1 & 110.9 & 117.0 \\
\hline P34371 & $\begin{array}{l}\text { Ethylbenzene, water, unfiltered, recoverable, } \\
\text { micrograms per liter }\end{array}$ & 96 & 73.6 & 78.7 & 83.3 & 88.6 & 98.0 & 104.9 & 125.0 \\
\hline P85795 & $\begin{array}{l}m \text {-Xylene plus } p \text {-xylene, water, unfiltered, } \\
\text { recoverable, micrograms per liter }\end{array}$ & 96 & 69.2 & 76.3 & 79.7 & 89.9 & 106.0 & 118.2 & 134.1 \\
\hline P34696 & $\begin{array}{l}\text { Naphthalene, water, unfiltered, recoverable, } \\
\text { micrograms per liter }\end{array}$ & 96 & 67.2 & 72.9 & 77.0 & 83.0 & 94.4 & 104.1 & 110.9 \\
\hline P77135 & $\begin{array}{l}o \text {-Xylene, water, unfiltered, recoverable, micro- } \\
\text { grams per liter }\end{array}$ & 96 & 79.0 & 80.6 & 84.5 & 90.5 & 100.4 & 107.0 & 126.2 \\
\hline P77350 & $\begin{array}{l}\text { sec-Butylbenzene, water, unfiltered, recover- } \\
\text { able, micrograms per liter }\end{array}$ & 96 & 72.6 & 77.7 & 80.5 & 88.9 & 100.0 & 108.2 & 126.9 \\
\hline P77128 & $\begin{array}{l}\text { Styrene, water, unfiltered, recoverable, micro- } \\
\text { grams per liter }\end{array}$ & 96 & 0.0 & 77.0 & 80.7 & 88.3 & 103.4 & 112.9 & 130.2 \\
\hline P78032 & $\begin{array}{l}\text { Methyl tert-butyl ether, water, unfiltered, recov- } \\
\text { erable, micrograms per liter }\end{array}$ & 96 & 73.4 & 92.0 & 94.9 & 98.8 & 103.4 & 109.4 & 125.5 \\
\hline P34475 & $\begin{array}{l}\text { Tetrachloroethene, water, unfiltered, recover- } \\
\text { able, micrograms per liter }\end{array}$ & 95 & 86.7 & 91.4 & 96.2 & 103.9 & 111.6 & 120.9 & 138.7 \\
\hline P32104 & $\begin{array}{l}\text { Tribromomethane, water, unfiltered, recover- } \\
\text { able, micrograms per liter }\end{array}$ & 96 & 80.0 & 84.4 & 88.4 & 92.2 & 99.4 & 107.2 & 133.9 \\
\hline P39180 & $\begin{array}{l}\text { Trichloroethene, water, unfiltered, recoverable, } \\
\text { micrograms per liter }\end{array}$ & 95 & 77.9 & 84.2 & 87.3 & 94.0 & 99.6 & 102.6 & 111.3 \\
\hline P32106 & $\begin{array}{l}\text { Trichloromethane, water, unfiltered, recover- } \\
\text { able, micrograms per liter }\end{array}$ & 93 & 89.9 & 96.0 & 100.5 & 104.6 & 107.9 & 112.8 & 121.5 \\
\hline P39175 & $\begin{array}{l}\text { Vinyl chloride, water, unfiltered, recoverable, } \\
\text { micrograms per liter }\end{array}$ & 96 & 73.0 & 81.6 & 89.1 & 96.4 & 103.8 & 110.0 & 140.5 \\
\hline P68726 & $\begin{array}{l}\text { 1,3-Butadiene, water, unfiltered, recoverable, } \\
\text { micrograms per liter }\end{array}$ & 96 & 4.4 & 71.3 & 78.8 & 89.8 & 102.6 & 112.7 & 131.7 \\
\hline P49538 & $\begin{array}{l}\text { 1,1-Difluoroethane, water, unfiltered, recover- } \\
\text { able, micrograms per liter }\end{array}$ & 96 & 72.0 & 81.3 & 85.1 & 90.7 & 96.3 & 101.2 & 108.9 \\
\hline P77323 & $\begin{array}{l}\text { 1,2,3,4-Tetrahydronaphthalene, water, unfil- } \\
\text { tered, recoverable, micrograms per liter }\end{array}$ & 96 & 58.0 & 65.3 & 75.3 & 84.3 & 107.0 & 115.5 & 127.7 \\
\hline
\end{tabular}


Table 4.7. Statistical summary of laboratory spike recovery results for volatile organic compounds included in laboratory schedule 4436.-Continued

[n, number of values for that constituent; min, minimum; 10th, 25th, 75th, and 90th, 10th, 25th, 75th, and 90th percentiles; med, median, max, maximum]

\begin{tabular}{|c|c|c|c|c|c|c|c|c|c|}
\hline \multirow{2}{*}{$\begin{array}{l}\text { Parameter } \\
\text { code }\end{array}$} & \multirow{2}{*}{ Constituent } & \multicolumn{8}{|c|}{ Statistic } \\
\hline & & $n$ & Min & 10th & 25th & Med & 75th & 90th & Max \\
\hline P50985 & $\begin{array}{l}\text { 1,2-Dichloro-1,1,2,2-tetrafluoroethane, water, } \\
\text { unfiltered, recoverable, micrograms per liter }\end{array}$ & 96 & 47.1 & 62.0 & 68.0 & 76.0 & 84.0 & 91.3 & 101.7 \\
\hline P85668 & $\begin{array}{l}\text { 1-Chloro-1,1-difluoroethane, water, unfiltered, } \\
\text { recoverable, micrograms per liter }\end{array}$ & 96 & 59.4 & 78.8 & 87.8 & 94.8 & 101.5 & 104.6 & 131.6 \\
\hline
\end{tabular}


Table 4.8. Statistical summary of laboratory spike recovery results for volatile organic compounds included in laboratory schedule 4437.

[ $n$, number of values for that constituent; min, minimum; 10th, 25th, 75th, and 90th, 10th, 25th, 75th, and 90th percentiles; med, median, max, maximum]

\begin{tabular}{|c|c|c|c|c|c|c|c|c|c|}
\hline \multirow{2}{*}{$\begin{array}{l}\text { Parameter } \\
\text { code }\end{array}$} & \multirow{2}{*}{ Constituent } & \multicolumn{8}{|c|}{ Statistic } \\
\hline & & $n$ & Min & 10th & 25th & Med & 75th & 90th & Max \\
\hline P80336 & $\begin{array}{l}\text { 1,1-Dichloro-2-propanone, water, unfiltered, recover- } \\
\text { able, micrograms per liter }\end{array}$ & 97 & 61.5 & 95.5 & 100.3 & 105.4 & 111.4 & 117.6 & 138.7 \\
\hline P81582 & $\begin{array}{l}\text { 1,4-Dioxane, water, unfiltered, recoverable, micro- } \\
\text { grams per liter }\end{array}$ & 97 & 69.6 & 90.1 & 93.6 & 97.5 & 102.0 & 106.3 & 114.5 \\
\hline P77076 & $\begin{array}{l}\text { 2-Nitropropane, water, unfiltered, recoverable, micro- } \\
\text { grams per liter }\end{array}$ & 97 & 64.1 & 92.8 & 95.3 & 98.6 & 103.7 & 107.5 & 117.4 \\
\hline P76997 & $\begin{array}{l}\text { Acetonitrile, water, unfiltered, recoverable, micrograms } \\
\text { per liter }\end{array}$ & 97 & 75.5 & 99.4 & 103.5 & 110.0 & 118.7 & 126.6 & 142.4 \\
\hline P34210 & $\begin{array}{l}\text { Acrolein, water, unfiltered, recoverable, micrograms } \\
\text { per liter }\end{array}$ & 77 & 0.0 & 81.6 & 87.4 & 94.6 & 103.8 & 108.4 & 118.2 \\
\hline P81578 & $\begin{array}{l}\text { Dimethoxymethane, water, unfiltered, recoverable, } \\
\text { micrograms per liter }\end{array}$ & 97 & 71.5 & 97.5 & 100.7 & 106.2 & 111.8 & 116.2 & 125.8 \\
\hline P81585 & $\begin{array}{l}\text { Ethyl acetate, water, unfiltered, recoverable, micro- } \\
\text { grams per liter }\end{array}$ & 97 & 60.7 & 88.4 & 94.3 & 101.5 & 107.0 & 110.9 & 125.8 \\
\hline P34386 & $\begin{array}{l}\text { Hexachlorocyclopentadiene, water, unfiltered, recover- } \\
\text { able, micrograms per liter }\end{array}$ & 97 & 46.7 & 71.1 & 79.2 & 89.3 & 108.4 & 121.2 & 149.8 \\
\hline P34408 & $\begin{array}{l}\text { Isophorone, water, unfiltered, recoverable, micrograms } \\
\text { per liter }\end{array}$ & 97 & 57.2 & 83.7 & 91.7 & 105.7 & 119.5 & 129.3 & 137.8 \\
\hline P77032 & $\begin{array}{l}\text { Methyl acetate, water, unfiltered, recoverable, micro- } \\
\text { grams per liter }\end{array}$ & 97 & 71.6 & 93.9 & 98.3 & 103.4 & 110.5 & 118.1 & 132.0 \\
\hline P34447 & $\begin{array}{l}\text { Nitrobenzene, water, unfiltered, recoverable, micro- } \\
\text { grams per liter }\end{array}$ & 97 & 54.9 & 82.5 & 89.3 & 97.2 & 106.5 & 118.0 & 133.6 \\
\hline P78200 & $\begin{array}{l}N \text {-Nitrosodiethylamine, water, unfiltered, recoverable, } \\
\text { micrograms per liter }\end{array}$ & 97 & 65.2 & 83.8 & 92.3 & 107.4 & 120.0 & 127.3 & 143.8 \\
\hline P77035 & $\begin{array}{l}\text { tert-Butyl alcohol, water, unfiltered, recoverable, mi- } \\
\text { crograms per liter }\end{array}$ & 97 & 64.9 & 94.6 & 97.8 & 100.8 & 106.3 & 111.8 & 119.6 \\
\hline P77443 & $\begin{array}{l}\text { 1,2,3-Trichloropropane, water, unfiltered, recoverable, } \\
\text { micrograms per liter }\end{array}$ & 97 & 64.1 & 92.4 & 97.2 & 100.0 & 104.6 & 108.4 & 117.2 \\
\hline P82625 & $\begin{array}{l}\text { 1,2-Dibromo-3-chloropropane, water, unfiltered, recov- } \\
\text { erable, micrograms per liter }\end{array}$ & 97 & 61.3 & 89.6 & 93.8 & 98.2 & 103.7 & 109.9 & 122.4 \\
\hline P77651 & $\begin{array}{l}\text { 1,2-Dibromoethane, water, unfiltered, recoverable, } \\
\text { micrograms per liter }\end{array}$ & 97 & 64.0 & 93.6 & 97.1 & 99.7 & 104.3 & 108.0 & 120.4 \\
\hline P34541 & $\begin{array}{l}\text { 1,2-Dichloropropane, water, unfiltered, recoverable, } \\
\text { micrograms per liter }\end{array}$ & 97 & 68.9 & 92.1 & 96.4 & 100.2 & 105.3 & 108.8 & 120.4 \\
\hline P78032 & $\begin{array}{l}\text { Methyl tert-butyl ether, water, unfiltered, recoverable, } \\
\text { micrograms per liter }\end{array}$ & 97 & 67.4 & 93.8 & 99.0 & 102.7 & 107.3 & 112.1 & 118.6 \\
\hline P68728 & $\begin{array}{l}\text { 2-Ethoxyethyl acetate, water, unfiltered, recoverable, } \\
\text { micrograms per liter }\end{array}$ & 97 & 72.7 & 93.7 & 100.6 & 110.4 & 120.3 & 126.7 & 135.1 \\
\hline P68729 & $\begin{array}{l}\text { 2-Propen-1-ol, water, unfiltered, recoverable, micro- } \\
\text { grams per liter }\end{array}$ & 97 & 68.4 & 93.8 & 99.7 & 107.0 & 114.3 & 118.4 & 125.1 \\
\hline P68730 & $\begin{array}{l}\alpha \text {-Terpineo, water, unfiltered, recoverable, micrograms } \\
\text { per liter }\end{array}$ & 97 & 51.9 & 78.0 & 87.0 & 98.4 & 113.2 & 126.2 & 145.1 \\
\hline P68732 & $\begin{array}{l}\text { Butanal, water, unfiltered, recoverable, micrograms per } \\
\text { liter }\end{array}$ & 97 & 69.3 & 87.3 & 90.2 & 95.5 & 102.9 & 107.4 & 120.4 \\
\hline P77548 & $\begin{array}{l}\text { Chloropicrin, water, unfiltered, recoverable, micro- } \\
\text { grams per liter }\end{array}$ & 97 & 67.1 & 92.9 & 99.8 & 105.1 & 114.7 & 123.1 & 133.8 \\
\hline
\end{tabular}


Table 4.8. Statistical summary of laboratory spike recovery results for volatile organic compounds included in laboratory schedule 4437.-Continued

[ $n$, number of values for that constituent; min, minimum; 10th, 25th, 75th, and 90th, 10th, 25th, 75th, and 90th percentiles; med, median, max, maximum]

\begin{tabular}{|c|c|c|c|c|c|c|c|c|c|}
\hline \multirow{2}{*}{$\begin{array}{l}\text { Parameter } \\
\text { code }\end{array}$} & \multirow{2}{*}{ Constituent } & \multicolumn{8}{|c|}{ Statistic } \\
\hline & & $n$ & Min & 10th & 25th & Med & 75th & 90th & Max \\
\hline P68733 & $\begin{array}{l}\text { trans-Crotonaldehyde, water, unfiltered, recoverable, } \\
\text { micrograms per liter }\end{array}$ & 97 & 73.5 & 87.7 & 92.9 & 96.9 & 101.7 & 106.2 & 115.8 \\
\hline P81583 & $\begin{array}{l}\text { 1,3-Dioxolane, water, unfiltered, recoverable, micro- } \\
\text { grams per liter }\end{array}$ & 97 & 75.1 & 93.3 & 98.2 & 103.3 & 108.9 & 113.5 & 122.2 \\
\hline P77034 & $\begin{array}{l}\text { 1-Butanol, water, unfiltered, recoverable, micrograms } \\
\text { per liter }\end{array}$ & 97 & 62.0 & 91.8 & 95.3 & 101.0 & 105.3 & 110.7 & 120.5 \\
\hline P68066 & $\begin{array}{l}\text { 1-Methoxy-4-(2-propenyl)benzene, water, unfiltered, } \\
\text { recoverable, micrograms per liter }\end{array}$ & 97 & 58.4 & 75.7 & 81.6 & 89.7 & 100.7 & 111.9 & 130.0 \\
\hline P77310 & $\begin{array}{l}\text { 1-Octanol, water, unfiltered, recoverable, micrograms } \\
\text { per liter }\end{array}$ & 97 & 56.5 & 79.9 & 84.5 & 89.9 & 94.9 & 100.4 & 115.7 \\
\hline P77419 & $\begin{array}{l}\text { 2,6-Dimethyl-4-heptanone, water, unfiltered, recover- } \\
\text { able, micrograms per liter }\end{array}$ & 97 & 53.8 & 81.5 & 85.3 & 90.2 & 97.2 & 103.3 & 116.9 \\
\hline P77311 & $\begin{array}{l}\text { 2-Ethyl-1-hexanol, water, unfiltered, recoverable, } \\
\text { micrograms per liter }\end{array}$ & 97 & 51.6 & 79.1 & 84.0 & 89.7 & 96.0 & 102.3 & 112.8 \\
\hline P77201 & $\begin{array}{l}\text { 2-Methylpropyl acetate, water, unfiltered, recoverable, } \\
\text { micrograms per liter }\end{array}$ & 97 & 60.2 & 79.9 & 84.3 & 88.3 & 93.6 & 97.2 & 109.1 \\
\hline P77113 & $\begin{array}{l}\text { 4-Methyl-2-pentanol, water, unfiltered, recoverable, } \\
\text { micrograms per liter }\end{array}$ & 97 & 60.1 & 89.1 & 93.3 & 97.3 & 102.0 & 104.7 & 109.5 \\
\hline P77179 & $\begin{array}{l}\text { 5-Methyl-2-hexanone, water, unfiltered, recoverable, } \\
\text { micrograms per liter }\end{array}$ & 97 & 60.7 & 89.1 & 93.1 & 98.8 & 104.5 & 108.0 & 115.0 \\
\hline P77097 & $\begin{array}{l}\text { Cyclohexanone, water, unfiltered, recoverable, micro- } \\
\text { grams per liter }\end{array}$ & 97 & 64.8 & 88.8 & 92.9 & 98.5 & 104.8 & 111.7 & 168.3 \\
\hline P45013 & $\begin{array}{l}\text { Isopropyl acetate, water, unfiltered, recoverable, micro- } \\
\text { grams per liter }\end{array}$ & 97 & 67.6 & 94.2 & 99.9 & 103.3 & 108.1 & 113.5 & 125.4 \\
\hline P77015 & $\begin{array}{l}\text { Isopropyl alcohol, water, unfiltered, recoverable, mi- } \\
\text { crograms per liter }\end{array}$ & 97 & 64.9 & 89.0 & 93.5 & 97.6 & 103.1 & 110.9 & 332.4 \\
\hline P77061 & $\begin{array}{l}n \text {-Pentanal, water, unfiltered, recoverable, micrograms } \\
\text { per liter }\end{array}$ & 97 & 63.9 & 85.3 & 88.9 & 93.1 & 97.3 & 102.2 & 115.5 \\
\hline P45022 & $\begin{array}{l}\text { Propyl acetate, water, unfiltered, recoverable, micro- } \\
\text { grams per liter }\end{array}$ & 97 & 69.3 & 85.6 & 89.0 & 93.9 & 100.1 & 105.8 & 118.2 \\
\hline
\end{tabular}


Table 4.9. Statistical summary of field spike recovery results for arsenic species analyzed under laboratory code 3142 .

[ $n$, number of values for that constituent; min, minimum; 10th, 25th, 75th, and 90th, 10th, 25th, 75th, and 90th percentiles; med, median, max, maximum]

\begin{tabular}{|c|c|c|c|c|c|c|c|c|c|}
\hline \multirow{2}{*}{$\begin{array}{l}\text { Parameter } \\
\text { code }\end{array}$} & \multirow{2}{*}{ Constituent } & \multicolumn{8}{|c|}{ Statistic } \\
\hline & & $n$ & Min & 10th & 25th & Med & 75th & 90th & Max \\
\hline P62453 & $\begin{array}{l}\text { Arsenate }\left(\mathrm{H}_{2} \mathrm{AsO}_{4}^{-}\right) \text {, water, filtered, micrograms per liter } \\
\text { as arsenic }\end{array}$ & 7 & 77.3 & 82.8 & 86.6 & 88.0 & 97.8 & 113.2 & 124.7 \\
\hline P62452 & $\begin{array}{l}\text { Arsenite }\left(\mathrm{H}_{3} \mathrm{AsO}_{3}\right) \text {, water, filtered, micrograms per liter } \\
\text { as arsenic }\end{array}$ & 9 & 60.6 & 79.1 & 84.9 & 90.6 & 97.4 & 111.0 & 111.2 \\
\hline P62455 & $\begin{array}{l}\text { Dimethylarsinate }\left(\left[\mathrm{CH}_{3}\right] 2 \mathrm{HAsO}_{2}\right) \text {, water, filtered, recov- } \\
\text { erable, micrograms per liter as arsenic }\end{array}$ & 9 & 63.6 & 68.4 & 87.3 & 89.9 & 93.8 & 99.3 & 100.0 \\
\hline P62454 & $\begin{array}{l}\text { Monomethylarsonate }\left(\left[\mathrm{CH}_{3}\right] \mathrm{HAsO}_{3}^{-}\right) \text {, water, filtered, } \\
\text { recoverable, micrograms per liter as arsenic }\end{array}$ & 9 & 70.6 & 79.3 & 83.1 & 85.3 & 96.9 & 100.2 & 103.3 \\
\hline
\end{tabular}




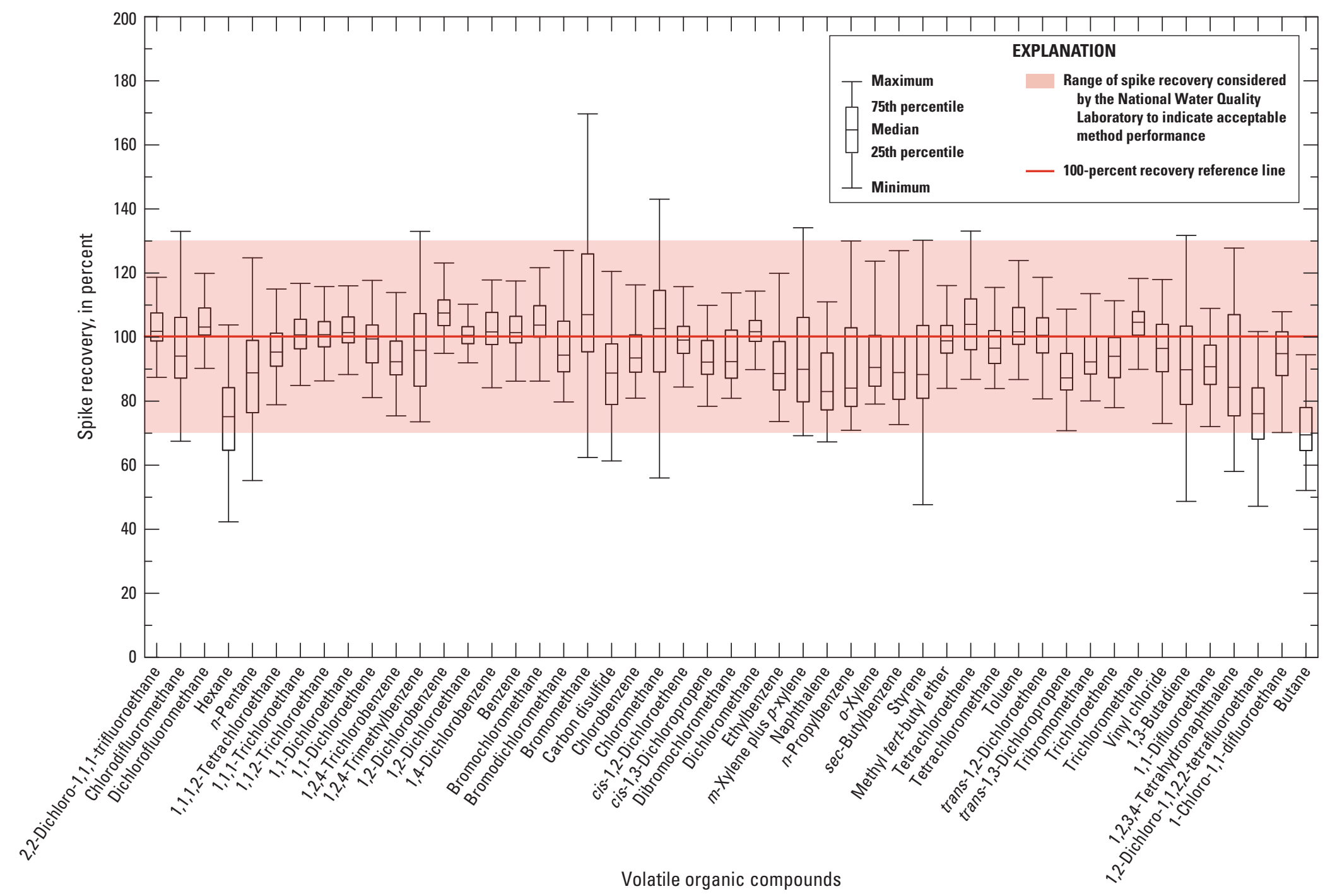

Figure 4.1. Laboratory spike recovery results for volatile organic compounds included in laboratory schedule 4436 . 


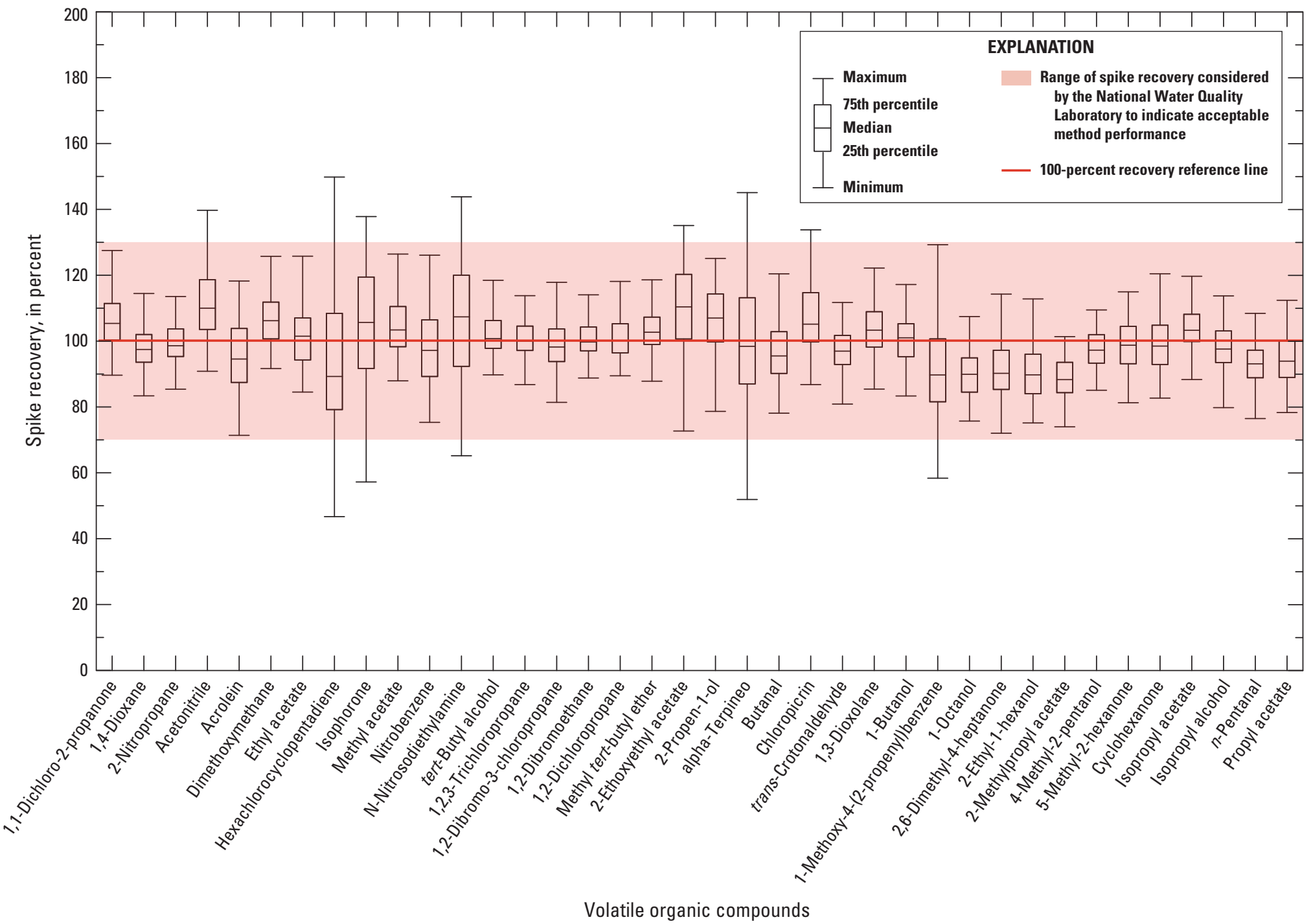

Figure 4.2. Laboratory spike recovery results for volatile organic compounds included in laboratory schedule 4437. 


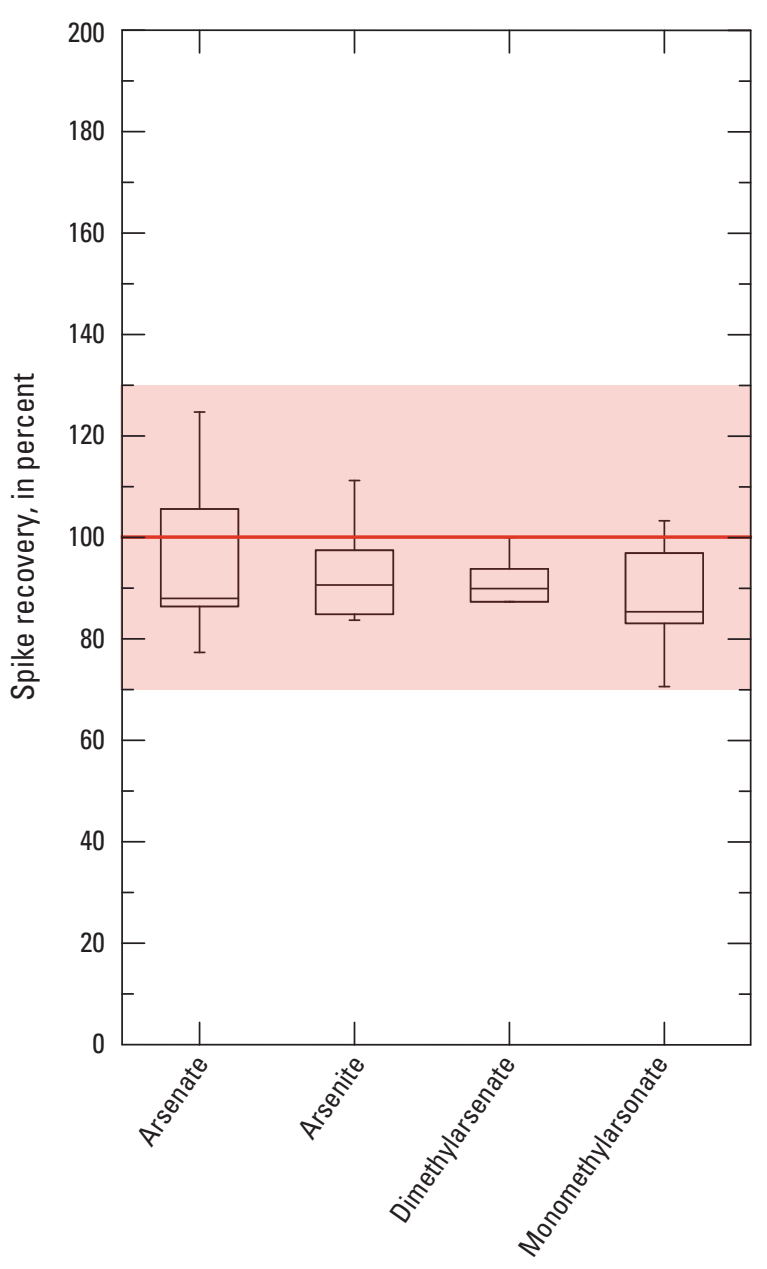

Arsenic species

\section{EXPLANATION}

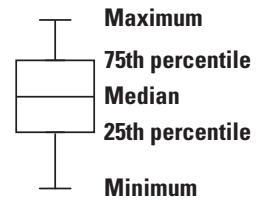

Range of spike recovery considered by the National Water Quality Laboratory to indicate acceptable method performance

- 100-percent recovery reference line

Figure 4.3. Field spike recovery results for arsenic speciation by laboratory code 3142 .

\section{References Cited}

Arnold, T.L., Bexfield, L.M., Musgrove, M., Lindsey, B.D., Stackelberg, P.E., Barlow, J.R., DeSimone, L.A., Kulongoski, J.T., Kingsbury, J.A., Ayotte, J.D., Fleming, B.J., and Belitz, K., 2017a, Datasets from groundwater quality data from the National Water Quality Assessment Project, January through December 2014 and select qualitycontrol data from May 2012 through December 2014: U.S. Geological Survey data release, accessed March 28, 2018, at https://doi.org/10.5066/F7W0942N.

Arnold, T.L., Bexfield, L.M., Musgrove, M., Lindsey, B.D., Stackelberg, P.E., Barlow, J.R., DeSimone, L.A., Kulongoski, J.T., Kingsbury, J.A., Ayotte, J.D., Fleming, B.J., and Belitz, K., 2017b, Groundwater-quality data from the National Water-Quality Assessment Project, January through December 2014 and select quality-control data from May 2012 through December 2014: U.S. Geological Survey Data Series 1063, 83 p., accessed March 28, 2018, at https://doi.org/10.3133/ds1063.

Arnold, T.L., Bexfield, L.M., Musgrove, M., Stackelberg, P.E., Lindsey, B.D., Kingsbury, J.A., Kulongoski, J.T., and Belitz, K., 2018b, Groundwater-quality and select qualitycontrol data from the National Water-Quality Assessment Project, January through December 2015, and previously unpublished data from 2013 to 2014: U.S. Geological Survey Data Series 1087, 68 p., accessed February 2020 at https://doi.org/10.3133/ds1087.

Arnold, T.L., Bexfield, L.M., Musgrove, M., Stackelberg, P.E., Lindsey, B.D., Kingsbury, J.A., Kulongoski, J.T., Belitz, K., and Sharpe, J.B., 2018a, Datasets from groundwaterquality and select quality-control data from the National Water-Quality Assessment Project, January through December 2015 and previously unpublished data from 2013-2014: U.S. Geological Survey data release, accessed February 2020 at https://doi.org/10.5066/F7XK8DHK.

Arnold, T.L., Bexfield, L.M., Musgrove, M., Stackelberg, P.E., Lindsey, B.D., Kingsbury, J.A., Kulongoski, J.T., Belitz, K., and Sharpe, J.B., 2020, Datasets from groundwaterquality and select quality-control data from the National Water-Quality Assessment Project, January through December 2016, and previously unpublished data from 2013 to 2015: U.S. Geological Survey data release, accessed February 2020 at https://doi.org/10.5066/P9W4RR74.

Arnold, T.L., DeSimone, L.A., Bexfield, L.M., Lindsey, B.D., Barlow, J.R., Kulongoski, J.T., Musgrove, M., Kingsbury, J.A., and Belitz, K., 2016b, Groundwater quality data from the National Water-Quality Assessment Project, May 2012 through December 2013: U.S. Geological Survey Data Series 997, 56 p., accessed March 28, 2018, at https://doi.org/10.3133/ds997. 
Arnold, T.L., DeSimone, L.A., Bexfield, L.M., Lindsey, B.D., Barlow, J.R., Kulongoski, J.T., Musgrove, M., Kingsbury, J.A., and Belitz, K., 2016a, Groundwater quality data from the National Water Quality Assessment Project, May 2012 through December 2014 and select quality-control data from May 2012 through December 2013: U.S. Geological Survey data release, accessed March 28, 2018, at https://doi.org/ 10.5066/F7HQ3X18.

Bender, D.A., Zagorsky, J.S., Mueller, D.K., Rose, D.L., Martin, J.D., and Brenner, C.K., 2011, Quality of volatile organic compound data from groundwater and surface water for the National Water-Quality Assessment Program, October 1996-December 2008: U.S. Geological Survey Scientific Investigations Report 2011-5204, 128 p., accessed January 4, 2016, at https://pubs.usgs.gov/sir/ 2011/5204/.

Davis, T.A., Olsen, L.D., Fram, M.S., and Belitz, K., 2014, Updated study reporting levels (SRLs) for trace-element data collected for the California Groundwater Ambient Monitoring and Assessment (GAMA) Priority Basin Project, October 2009-March 2013: U.S. Geological Survey Scientific Investigations Report 2014-5105, 52 p., accessed January 4, 2016, at https://doi.org/10.3133/sir20145105.

Fram, M.S., Olsen, L.D., and Belitz, K., 2012, Evaluation of volatile organic compound (VOC) blank data and application of study reporting levels to groundwater data collected for the California GAMA Priority Basin Project, May 2004 through September 2010: U.S. Geological Survey Scientific Investigations Report 2012-5139, 94 p., accessed January 4, 2016, at https://pubs.usgs.gov/sir/2012/5139/.

Gilliom, R.J., Barbash, J.E., Crawford, C.G., Hamilton, P.A., Martin, J.D., Nakagaki, N., Nowell, L.H., Scott, J.C., Stackelberg, P.E., Thelin, G.P., and Wolock, D.M., 2006, The quality of our Nation's waters-Pesticides in the Nation's streams and ground water, 1992-2001: U.S. Geological Survey Circular 1291, accessed January 4, 2016, at https://pubs.usgs.gov/circ/2005/1291/.
Martin, J.D., 2002, Variability of pesticide detections and concentrations in field replicate water samples collected for the National Water-Quality Assessment Program, 1992-97: U.S. Geological Survey Water-Resources Investigations Report 01-4178, 84 p., accessed June 30, 2016, at https:/pubs.usgs.gov/wri/2001/wri01_4178/.

Mueller, D.K., Schertz, T.L., Martin, J.D., and Sandstrom, M.W., 2015, Design, analysis, and interpretation of field quality-control data for water-sampling projects: U.S. Geological Survey Techniques and Methods, book 4, chap. C4, 54 p. [Also available at https://doi.org/10.3133/ tm4C4.]

Mueller, D.K., and Titus, C.J., 2005, Quality of nutrient data from streams and ground water sampled during water years 1992-2001: U.S. Geological Survey Scientific Investigations Report 2005-5106, 27 p. [Also available at https://doi.org/10.3133/sir20055106.]

Olsen, L.D., Fram, M.S., and Belitz, K., 2010, Review of trace-element field-blank data collected for the California Groundwater Ambient Monitoring and Assessment (GAMA) Program, May 2004-January 2008: U.S. Geological Survey Scientific Investigations Report 2009-5220, 47 p., accessed January 4, 2016, at https://pubs.usgs.gov/sir/2009/5220/.

Shoda, M.E., Nowell, L.H., Stone, W.W., Sandstrom, M.W., and Bexfield, L.M., 2018, Data analysis considerations for pesticides determined by National Water Quality Laboratory schedule 2437: U.S. Geological Survey Scientific Investigations Report 2018-5007, 458 p., accessed February 2020 at https://doi.org/10.3133/sir20185007.

Zogorski, J.S., Carter, J.M., Ivahnenko, T., Lapham, W.W., Moran, M.J., Rowe, B.L., Squillace, P.J., and Toccalino, P.L., 2006, The quality of our Nation's waters - Volatile organic compounds in the Nation's groundwater and drinking-water supply wells: U.S. Geological Survey Circular 1292, 101 p., accessed January 4, 2016, at https://pubs.usgs.gov/circ/circ1292/. 
For more information about this publication, contact:

Director, USGS Central Midwest Water Science Center

405 North Goodwin

Urbana, IL 61801

217-328-8747

For additional information, visit: https://www.usgs.gov/centers/cm-water

Publishing support provided by the

Rolla Publishing Service Center 



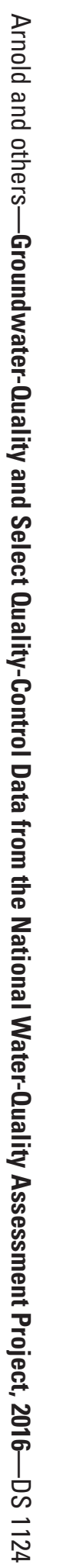

